\title{
DISCOURSE, CONVERSATION AND ARGUMENTATION: THEORETICAL PERSPECTIVES AND INNOVATIVE EMPIRICAL STUDIES - VOLUME I
}

EDITED BY: Antonio Bova, Carlo Galimberti, Lise Haddouk and Francesco Arcidiacono

PUBLISHED IN: Frontiers in Psychology and Frontiers in Communication

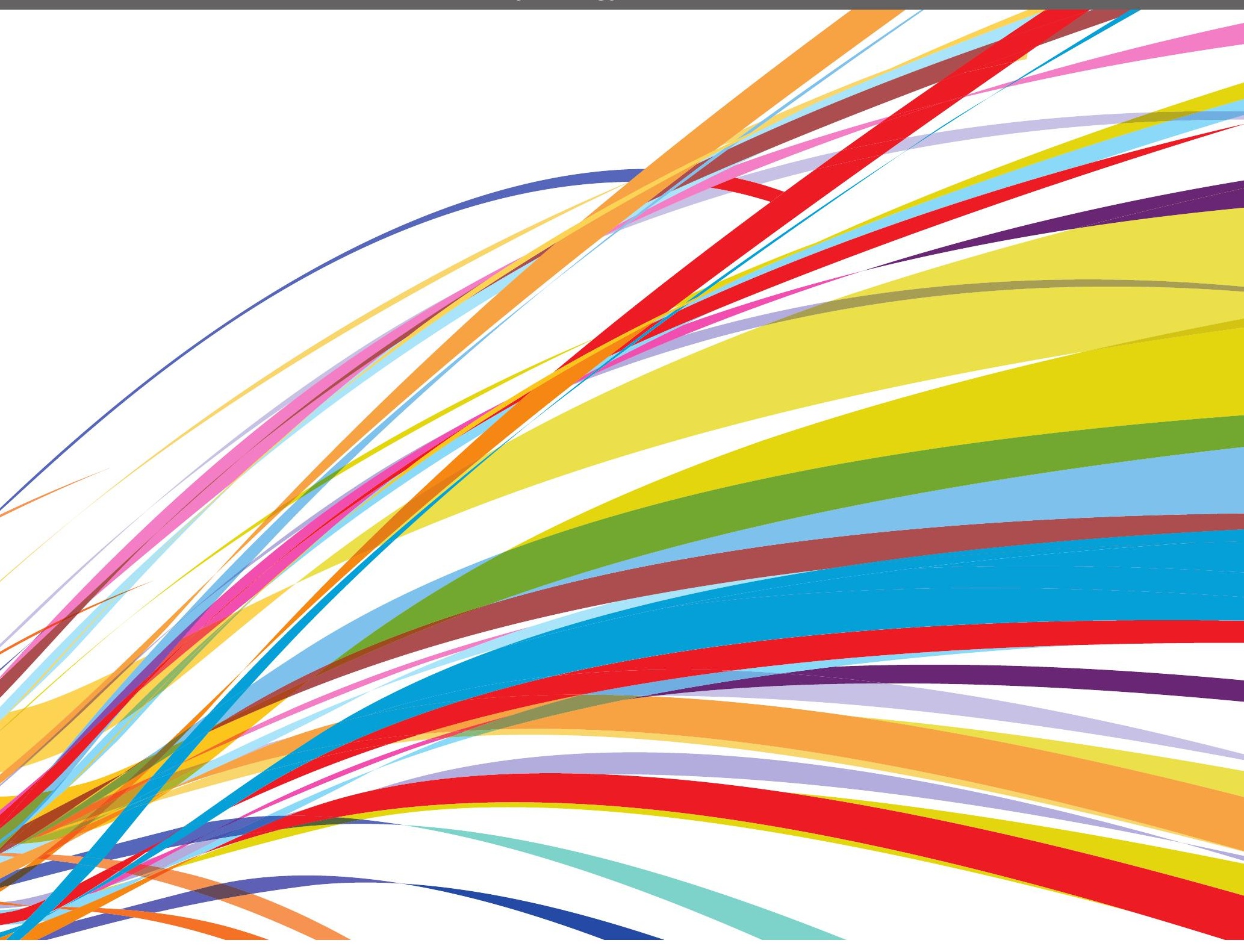

frontiers Research Topics 


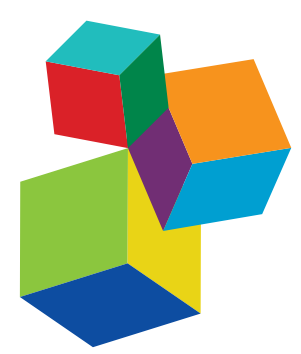

Frontiers eBook Copyright Statement

The copyright in the text of individual articles in this eBook is the property of their respective authors or their respective institutions or funders. The copyright in graphics and images within each article may be subject to copyright of other parties. In both cases this is subject to a license granted to Frontiers.

The compilation of articles constituting this eBook is the property of Frontiers.

Each article within this eBook, and the eBook itself, are published under the most recent version of the Creative Commons CC-BY licence. The version current at the date of publication of this eBook is CC-BY 4.0. If the CC-BY licence is updated, the licence granted by Frontiers is automatically updated to the new version.

When exercising any right under the CC-BY licence, Frontiers must be attributed as the original publisher of the article or eBook, as applicable.

Authors have the responsibility of ensuring that any graphics or other materials which are the property of others may be included in the

CC-BY licence, but this should be checked before relying on the

CC-BY licence to reproduce those materials. Any copyright notices relating to those materials must be complied with.

Copyright and source acknowledgement notices may not be removed and must be displayed in any copy, derivative work or partial copy which includes the elements in question.

All copyright, and all rights therein, are protected by national and international copyright laws. The above represents a summary only.

For further information please read Frontiers' Conditions for Website

Use and Copyright Statement, and the applicable CC-BY licence.

ISSN 1664-8714

ISBN 978-2-88971-803-0

DOI 10.3389/978-2-88971-803-0

\section{About Frontiers}

Frontiers is more than just an open-access publisher of scholarly articles: it is a pioneering approach to the world of academia, radically improving the way scholarly research is managed. The grand vision of Frontiers is a world where all people have an equal opportunity to seek, share and generate knowledge. Frontiers provides immediate and permanent online open access to all its publications, but this alone is not enough to realize our grand goals.

\section{Frontiers Journal Series}

The Frontiers Journal Series is a multi-tier and interdisciplinary set of open-access, online journals, promising a paradigm shift from the current review, selection and dissemination processes in academic publishing. All Frontiers journals are driven by researchers for researchers; therefore, they constitute a service to the scholarly community. At the same time, the Frontiers Journal Series operates on a revolutionary invention, the tiered publishing system, initially addressing specific communities of scholars, and gradually climbing up to broader public understanding, thus serving the interests of the lay society, too.

\section{Dedication to Quality}

Each Frontiers article is a landmark of the highest quality, thanks to genuinely collaborative interactions between authors and review editors, who include some of the world's best academicians. Research must be certified by peers before entering a stream of knowledge that may eventually reach the public - and shape society; therefore, Frontiers only applies the most rigorous and unbiased reviews.

Frontiers revolutionizes research publishing by freely delivering the most outstanding research, evaluated with no bias from both the academic and social point of view. By applying the most advanced information technologies, Frontiers is catapulting scholarly publishing into a new generation.

\section{What are Frontiers Research Topics?}

Frontiers Research Topics are very popular trademarks of the Frontiers Journals Series: they are collections of at least ten articles, all centered on a particular subject. With their unique mix of varied contributions from Original Research to Review Articles, Frontiers Research Topics unify the most influential researchers, the latest key findings and historical advances in a hot research area! Find out more on how to host your own Frontiers Research Topic or contribute to one as an author by contacting the Frontiers Editorial Office: frontiersin.org/about/contact 


\section{DISCOURSE, CONVERSATION AND ARGUMENTATION: THEORETICAL PERSPECTIVES AND INNOVATIVE EMPIRICAL STUDIES - VOLUME I}

Topic Editors:

Antonio Bova, Catholic University of the Sacred Heart, Italy Carlo Galimberti, Catholic University of the Sacred Heart, Italy Lise Haddouk, Université de Rouen, France

Francesco Arcidiacono, Haute École Pédagogique BEJUNE, Switzerland

Citation: Bova, A., Galimberti, C., Haddouk, L., Arcidiacono, F., eds. (2021). Discourse, Conversation and Argumentation: Theoretical Perspectives and Innovative Empirical Studies - Volume I. Lausanne: Frontiers Media SA. doi: 10.3389/978-2-88971-803-0 


\section{Table of Contents}

04 Editorial: Discourse, Conversation and Argumentation: Theoretical Perspectives and Innovative Empirical Studies-Volume I

Francesco Arcidiacono, Antonio Bova, Carlo Galimberti and Lise Haddouk

06 Embodied Displays of "Doing Thinking." Epistemic and Interactive Functions of Thinking Displays in Children's Argumentative Activities Vivien Heller

27 The Psychophysiological Experience of Solving Moral Dilemmas Together: An Interdisciplinary Comparison Between Participants With and Without Depression

Emmi Koskinen, Samuel Tuhkanen, Milla Järvensivu, Enikö Savander, Taina Valkeapää, Kaisa Valkia, Elina Weiste and Melisa Stevanovic

43 "Argue With Me": A Method for Developing Argument Skills Kalypso lordanou and Chrysi Rapanta

57 Being in Place: A Multimodal Analysis of the Contribution of the Patient's Companion to "First Time" Oncological Visits Marilena Fatigante, Cristina Zucchermaglio and Francesca Alby

80 Emotional Tension as a Frame for Argumentation and Decision-Making: Vegetarian vs. Omnivorous Diets María Pilar Jiménez-Aleixandre and Pablo Brocos

95 Deliberative Teaching as an Emergent Field: The Challenge of Articulating Diverse Research Agendas to Promote Educational Experiences for Citizenship

Antonia Larrain, Gabriel Fortes and María Teresa Rojas

110 Polydimensional Structure and Psychosocial Functions of the Direct Address in TV Series

Carlo Galimberti, Antonio Bova, Carmen Spanò and Ilaria Vergine

121 The Dilemma of Inclusive Education: Inclusion for Some or Inclusion for All Äli Leijen, Francesco Arcidiacono and Aleksandar Baucal 


\title{
Editorial: Discourse, Conversation and Argumentation: Theoretical Perspectives and Innovative Empirical Studies - Volume I
}

\author{
Francesco Arcidiacono ${ }^{1 * t}$, Antonio Bova ${ }^{2}$, Carlo Galimberti ${ }^{2}$ and Lise Haddouk ${ }^{3}$ \\ ${ }^{1}$ Research Department, Haute Ecole Pédagogique - Cantons de Berne Francophone, Jura et Neuchâtel, Biel/Bienne, \\ Switzerland, ${ }^{2}$ Department of Psychology, Catholic University of the Sacred Heart, Milan, Italy, ${ }^{3}$ Department of Psychology, \\ Université de Rouen, Mont-Saint-Aignan, France
}

Keywords: argumentation, communicative interactions, conversation, discourse, psychology

Editorial on the Research Topic

Discourse, Conversation and Argumentation: Theoretical Perspectives and Innovative Empirical Studies-Volume I

Thinking about how we participate in an interaction through verbal and non-verbal exchanges is a way to focus on explicit and implicit norms, personal and collective preferences, subjective and interpersonal theories, and social processes of construction of meaning that characterize our everyday communicative interactions. Although discursive, conversational, and argumentative interactions play an essential role in our lives, there is no integrated area of psychological research on these types of communicative interactions. A wide variety of works is available concerning the focus on the different roles played by social actors within the interactions (symmetric-asymmetric, protagonist-antagonist, and teacher-learner), as well as the interest for the constitutive (emotional, motivational, and cognitive) aspects of the interactions or developmental factors (skills, competences, and knowledge). However, research on these topics is conducted in a number of separate research communities that are spread across disciplines and have only limited intertwinements. This Research Topic intends to offer a dialogical platform for sharing studies on discourse, conversation, and argumentation from a psychological perspective. Different strands were bridged together to reach two main goals:

Specialty section: This article was submitted to

Language Sciences,

a section of the journal

Frontiers in Psychology

Received: 13 September 2021

Accepted: 27 September 2021

Published: 22 October 2021

Citation:

Arcidiacono F, Bova A, Galimberti C and Haddouk L (2021) Editorial:

Discourse, Conversation and

Argumentation: Theoretical

Perspectives and Innovative Empirical

Studies-Volume I.

Front. Psychol. 12:775053.

doi: 10.3389/fpsyg.2021.775053

(1) to explore novel and promising theoretical perspectives to study discursive, conversational, and argumentative interactions from a psychological perspective;

(2) and provide an extensive platform of the latest innovative research investigating interactions between individuals, groups, and institutions.

The Research Topic provided an opportunity for researchers working in different international and psychological contexts to draw together their work within a common forum. Through their contributions, authors provided state-of-the-art of collective evidences in psychological research on discourse, conversation, and argumentation. They were able to place emphasis on opportunities for mutual awareness and integration by proposing a series of high-quality original research papers, reviews, and conceptual analyses on topics dealing with the above-mentioned issues. The contributions are focused on psychological perspectives on interactions and empirically supported approaches to analyze them. This panel of respectful researchers contributed to render the Research Topic particularly timely and open to colleagues who are continually exposed to nearly limitless sectorial approaches. In this vein, we hope that the contributions can be challenging for a large 
scientific audience to support integrated psychological research on discursive, conversational, and argumentative interactions.

The paper of Koskinen et al. presents an original research offering a better understanding of the affective corollaries of social interactions during a psychophysiological experience of solving moral dilemmas. The authors compared participants with and without depression and showed their positions in expressing agreements and disagreement while solving moral dilemmas together.

Heller proposes a study on embodied displays of "doing thinking" by analyzing the epistemic and interactive functions of thinking displays in children's argumentative activities. From an interactional perspective, her paper investigates moments in which one participant in an interaction embodies displays of doing thinking as a recurring social practice serving interactive functions. The activities of joint decision-making are considered the functional argumentative setting to reach two interrelated aims: the first is to describe how multiple modalities (gaze and bodily postures) are temporally coordinated to create multimodal gestalts of doing thinking; the second one is to generate knowledge about the functions of thinking displays in children's argumentative activities.

Jiménez-Aleixandre and Brocos propose another original paper to highlight the relationship between argumentative scientific evidences and emotions. In particular, the authors focus on the exam of the ways through which emotional tensions frame the construction of arguments about vegetarian vs. omnivorous diets within a group of preservice teachers. Their investigation shows how the interactions between the group emotional tension and the evaluation of evidence drive a change toward a decision that would be emotionally acceptable for all participants.

The paper proposed by Galimberti et al. considers the direct address as a research object within the field of social psychology of communication. Their research contributes to increase our understanding of this technique by going beyond the analysis of its dramaturgical function (through the consideration of a TV series). In fact, the authors propose an integrated approach based on argumentative and conversational tools, showing that the direct address performs its dramaturgical function by impacting both diegetic and extradiegetic levels.

Another original research is proposed by Fatigante et al., who analyze how the companion participation during first oncological visits is a local and sequential accomplishment, changing from time to time in the consultation. The paper focuses on how patient's companions orient and contribute to the accomplishment of the different aims and activities at different stages of the visit as an institutional speech event. The authors refer to a multimodal analysis of turns and actions to closely examine the sequential and temporal arrangements of companions' and their co-participants' turns.
A conceptual analysis is provided by Leijen et al. to consider different understandings of inclusive education that frame current public and professional debates as well as policies and practices. The authors analyze two discourses regarding inclusive education by reconstructing the inferential configurations of the arguments of each narrative. The paper contributes to identify how the two definitions position children with and without special needs and their teachers.

Iordanou and Rapanta propose a review about a method for developing argument skills. The authors examine a particular program, the "Argue with $\mathrm{Me}$ " dialogue-based pedagogical approach, not only as a tool for supporting the development of argument skills but also the way of how empirical research employing the method in varying contexts provides insights into the nature of argument skills and their development, as well as the relations between argument skills and other skills or forms of understanding.

Another review (Larrain et al.) considers the relevance of deliberative education for contemporaneous democracies and citizenship, seeking to converge in a field of interlocution, calling it deliberative teaching. The paper proposes a way to increase the dialog and collaboration between the diffuse literature on argumentation and education. The authors highlight both the main theoretical and empirical gaps and challenges, as well as the possibilities to advance our knowledge and the educational impact that this integrating field could offer.

\section{AUTHOR CONTRIBUTIONS}

All authors listed have made a substantial, direct and intellectual contribution to the editorial, and approved it for publication.

Conflict of Interest: The authors declare that the research was conducted in the absence of any commercial or financial relationships that could be construed as a potential conflict of interest.

Publisher's Note: All claims expressed in this article are solely those of the authors and do not necessarily represent those of their affiliated organizations, or those of the publisher, the editors and the reviewers. Any product that may be evaluated in this article, or claim that may be made by its manufacturer, is not guaranteed or endorsed by the publisher.

Copyright (C) 2021 Arcidiacono, Bova, Galimberti and Haddouk. This is an open-access article distributed under the terms of the Creative Commons Attribution License (CC BY). The use, distribution or reproduction in other forums is permitted, provided the original author(s) and the copyright owner(s) are credited and that the original publication in this journal is cited, in accordance with accepted academic practice. No use, distribution or reproduction is permitted which does not comply with these terms. 
OPEN ACCESS

Edited by:

Antonio Bova,

Catholic University of the Sacred

Heart, Italy

Reviewed by:

Josephine Convertini,

University of Applied Sciences and

Arts of Southern Switzerland

(SUPSI), Switzerland

Sara Greco,

University of Italian

Switzerland, Switzerland

*Correspondence:

Vivien Heller

vheller@uni-wuppertal.de

Specialty section:

This article was submitted to

Language Sciences,

a section of the journal

Frontiers in Psychology

Received: 01 December 2020

Accepted: 27 January 2021

Published: 18 February 2021

Citation:

Heller V (2021) Embodied Displays of

"Doing Thinking." Epistemic and

Interactive Functions of Thinking

Displays in Children's Argumentative

Activities. Front. Psychol. 12:636671.

doi: 10.3389/fpsyg.2021.636671

\section{Embodied Displays of "Doing Thinking." Epistemic and Interactive Functions of Thinking Displays in Children's Argumentative Activities}

\author{
Vivien Heller* \\ Department of German Studies, School of Humanities and Cultural Studies, University of Wuppertal, Wuppertal, Germany
}

This study investigates moments in which one participant in an interaction embodies that he is "doing thinking," a display that is commonly referred to as "thinking face. " From an interactional perspective, it is assumed that embodied displays of "doing thinking" are a recurring social practice and serve interactive functions. While previous studies have examined thinking faces primarily in word searches and storytelling, the present study focuses on argumentative activities, in which children engage in processes of joint decision-making. The paper has two interrelated aims. The first aim is to describe how multiple modalities - beyond the face-are temporally coordinated to create multimodal gestalts of "doing thinking." It is shown that thinking displays not only involve dynamic imaginative gaze but also stylized bodily postures. The second aim is to generate knowledge about the functions of thinking displays in children's argumentative activities. The analysis describes how both speakers and recipients use thinking displays in different turn positions and align them with verbal talk or silence. The data for this study comprise video recordings of decision-making processes in groups of older children. Drawing on a multimodal approach to situated interaction, it will be proposed that embodied displays of "doing thinking" provide a resource to shape participation frameworks, mark epistemic stances and create epistemic ecologies for collaborative reasoning. By investigating thinking displays in a particular conversational activity, the study sheds light on the diversity and context-sensitive functionality of thinking displays. It also contributes to recent research on children's collaborative reasoning as an embodied discursive practice.

Keywords: thinking face, multimodal gestalts, posture, gaze, epistemic stance, argumentation, decision-making, conversation analysis

\section{INTRODUCTION}

Facial expressions are an integral part of face-to-face interaction and shape the way we interpret the actions and stances of our interlocutors. Research disagrees as to whether facial expressions represent a mere epiphenomenon of interaction or an interactive resource. They are thus conceptualized either as an externalization of emotional and cognitive states or as interactive resources. From these different perspectives, various facial expressions were described in more 
detail, including smiles (Ekman and Friesen, 1975; Kaukomaa et al., 2013), frowns (Ekman, 1979; Kaukomaa et al., 2014), and so-called thinking faces (Goodwin and Goodwin, 1986; Bavelas and Chovil, 2018). The latter typically entail that speakers withdraw their gaze; in addition, the gaze is not focused on persons or objects in the immediate surroundings but instead assumes a "middle-distance" look. Previous studies have shown that thinking faces are often used in word searches and storytelling. In their seminal study on word searches, Goodwin and Goodwin (1986) revealed that in those moments, when the speaker is hesitating and speaking, the thinking face serves as a visible display of his continued involvement in the joint activity, preventing the co-participants from taking the turn. This research demonstrates that thinking faces have important interactive functions. So far, however, little is known about the role thinking faces play in other discursive practices and whether they are used not only by speakers but also by listeners. It is also noticeable that previous research has focused exclusively on the face, arguably because it plays a prominent role in displays of "doing thinking." However, such displays might involve other less prominent, but nevertheless relevant resources.

This paper addresses these issues and examines thinking faces in children's argumentative decision-making. Understanding argumentation as an interactively organized and embodied discursive practice (e.g., Mirivel, 2011; Jacquin, 2017; Heller, 2018) and drawing on a multimodal approach to situated interaction (Goodwin, 2000), the sequential analysis describes how both speakers and recipients combine various resources to create a complex multimodal gestalt that embodies "doing thinking." It is proposed that what will be called thinking postures together with imaginative gaze and vivid eye movement are constitutive components of these displays. Furthermore, it is shown that these displays frequently occur in hypothetical scenarios where they are combined with lexical, morphological and syntactical markers of epistemic stance. In these conversational contexts, embodied displays of "doing thinking" serve not only interactive but also epistemic functions. It will be argued that they contribute to organizing thinking as public practice and to creating epistemic ecologies for collaborative reasoning. Such ecologies can be considered essential for establishing the "jointness of emerging decisions" (Stevanovic et al., 2017) in argumentative processes.

I begin by discussing previous research on facial expressions, thinking faces, and argumentation as an embodied discursive practice. Subsequently, I present the data and explain the analytical approach to the description of embodied displays. The analysis is divided into two parts. The first section examines the displays of speakers, the second the displays of recipients. A discussion of the findings concludes the paper.

\section{THEORETICAL PERSPECTIVE}

As yet, thinking displays were conceptualized as a facial expression. Accordingly, Goodwin and Goodwin (1986) coined the term "thinking face." As an essential component of what Goffman calls "the personal front" (1963, p. 25), the face is an integral part of social interaction. Through facial movements participants show themselves "to be situationally present" (ibid., p. 27) and responsive to the obligations of their involvements with others. Compared to other body parts, the face is particularly mobile and flexible. As noted by Kidwell (2013, p. 104), these properties make it "an especially useful resource as both a standin for, and elaborator of, talk."

Previous research on facial expressions can be categorized into two major strands of research that approach facial expressions as an externalization of inner emotional or cognitive states (e.g., Darwin, 1872; Ekman and Friesen, 1969; Ricci Bitti, 2014) or as interactive resources (e.g., Birdwhistell, 1970; Kendon, 1976; Chovil, 1991; Peräkylä and Ruusuvuori, 2006; Bavelas et al., 2014; Crivelli and Fridlund, 2018). While the former tends to focus on the individual, the latter investigates facial expressions as a social phenomenon.

Although Ekman and colleagues examine facial expressions both as emotional expressions and as conversational signals, their focus is on the face as "the primary site of affect displays" (Ekman and Friesen, 1969, p. 71), i.e., on the ways in which "internal" emotional states are expressed and recognized in and through the face. The assumption is that emotional expressions have inherent and stable meanings whereas conversational signals only emphasize, underline, and modulate verbal talk. According to Ekman, emotional expressions are spontaneous and occur early in ontogenesis. In contrast, conversational signals are used intentionally and acquired only after children have developed some "intentional language" (Ekman, 1979, p. 191). Methodically, Ekman has approached the study of facial expression either through detailed description of the muscles that are involved in producing a specific facial expression or through judgement tasks based on photographs. As Goodwin et al. (2012, p. 17) clearly show, this approach has considerable shortcomings: the face is examined in isolation from the speaker's body and the bodies of the co-participants; second, the "unfolding flow of an action in interaction" is ignored.

Interactional traditions conceive facial expressions as "visible acts of meaning" and examine the ways in which they "are part of the integrated message with words" (Bavelas and Chovil, 2000, p. 166). Emphasizing the functional similarities between facial expressions and gestures, Bavelas et al. (2014, p. 16-17) adopt Kendon's (2004, p. 310) notion of "facial gesture" to refer to "any configuration or movement of the face or head (including the eyes) that is synchronized with speech in both timing and meaning." In addition, they apply Kendon's distinction between referential, interactive (or interpersonal), and pragmatic gestures to facial expressions. In a study on facial gestures in storytelling, Bavelas and Chovil (2018) observe that the majority of facial gestures serve pragmatic rather than referential functions.

While Bavelas and colleagues examine facial gestures by relating them to individual utterances, Peräkylä and Ruusuvuori (2006) investigate facial displays within the framework of Conversation Analysis. Inspired by the work on mutual monitoring and organization (Goodwin, 1980, 1981), they focus on the interplay and temporal organization of facial and other forms of expressions. Furthermore, they are interested in the role facial expressions play in different conversational activities, e.g., 
assessments (Peräkylä and Ruusuvuori, 2006) and storytelling (Ruusuvuori and Peräkylä, 2009; Peräkylä and Ruusuvuori, 2012). They show that facial expressions can project, accompany, and follow lexical elements that encode the speaker's stance and thus extend the boundaries of the spoken turn of talk. Furthermore, they examine how recipients respond to the speaker's facial stance displays and how they produce facial expressions themselves to shift the speaker's stance (Kaukomaa et al., 2015). This research demonstrates that facial expression is a highly flexible interactional resource that can be easily adapted to the contingencies of the activity-in-progress. Following this line of research, the present study analyzes embodied displays of "doing thinking" as social phenomena that are sequentially and interactively organized.

\section{The Thinking Face}

The thinking face is one of several displays with which participants enact "doing thinking" and convey their stance toward what is being said. For instance, (facial) shrugs are used to display a distanced, less than committed stance (Streeck, 2011, p. 189f.) or to claim that something is obvious (Kendon, 2004; p. 275). Raised eyebrows (together with other facial actions) are reported to display disbelief, mock astonishment, or sophisticated skepticism (Ekman, 1979, p. 188f.). Frowns provide a resource to mark something as problematic and thus help preserve intersubjectivity in problematic conversational moments (Kaukomaa et al., 2014).

Thinking faces have occasionally been mentioned in research, though under different terms. Darwin (1872, p. 228f.) noted the "vacant expression of the eyes" that typically occurs "when a man is completely lost in thought." He observes that the unfocused eyes can be accompanied by other movements or gestures, such as raising the hands to the forehead, mouth, or chin. Given the fact that Darwin did not have the opportunity to examine the temporal unfolding of interaction, it seems remarkable that he actually draws attention to a number of relevant components beyond facial expressions. Yet for him, this facial expression reflects an actual state of mind and is associated with processes of "abstraction" or "meditation" (ibid., p. 228).

Unlike Darwin, Goodwin and Goodwin (1986) conceptualize what they call "thinking face" not as the expression of an inner state, but as a sedimented and socially shared conversational resource. They observe that speakers who are involved in a word search withdraw their gaze and produce a characteristic, stereotypic thinking face. In such moments, the gaze is not focused on persons or objects in the immediate surroundings but instead assumes an "out of focus "middle-distance" look" (Goodwin, 1987, p. 117). Goodwin and Goodwin suggest that the thinking face is used as an interactional resource rather than being an adjustment to the cognitive demands that a word search entails: during a moment when the speaker is not speaking, the thinking face serves as a visible display of the speaker's continued involvement in the joint activity (storytelling). Through small changes in the facial expression and other resources such as fillers, pursing and slackening of the lips, opening or dropping the hand, and wh-questions such as "What the heck was it?", the speaker visualizes distinct stages in his search for a word.
In this way, the display works to prevent the co-participants from entering the unfinished turn. Furthermore, as speakers move through these stages, they can change the participation framework by resuming eye contact to solicit help from the recipients, thereby contextualizing the word search as "a visible activity that other participants not only recognize but can also participate in" (Goodwin and Goodwin, 1986, p. 52). This is why Bavelas et al. (2014) assume that thinking faces serve interactive functions as opposed to other pragmatic functions (i.e., modal, performative, parsing, cf. Kendon, 2004, p. 158f.). However, in another study on "remembering" as an interactional resource in storytelling, Goodwin (1987) conceptualizes thinking faces as displays of uncertainty. Thus, it seems that they can also serve modal functions, i.e., alter the frame in terms of which an utterance is to be interpreted.

Other studies examine thinking faces in elicited talk. Chovil (1991, 1997) studies the frequency with which different facial expressions occur in different activities (planning a meal, retelling a conflict, and a close-call experience). In her data, only speakers produce thinking faces. In what she calls "nonredundant" facial displays, thinking faces account for more than a quarter of all facial expressions. Furthermore, the multimodal composition of thinking faces varies slightly depending on the conversational context. In addition to withdrawing gaze and looking "thoughtful," speakers sometimes also "lower eyebrows in a frown, or raise them while looking off in the distance, close their eyes, pull one side of their mouth back or twist their mouth to one side" (ibid., 182f). Building on a study by Clark and Fox Tree (2002), who describe "uh" and "um" as "collateral signals," Bavelas and Chovil (2018) examine thinking faces in elicited telling. Thinking faces that occur at the beginning of the story are usually long. In addition, thinking faces are usually produced at transitions to other details or word searches. In most cases, they are introduced or accompanied by some form of verbal collateral signal; therefore, the authors suggest that the thinking face itself is a "collateral signal."

In summary, it can be noted that thinking faces serve the recipient as recognizable displays that the speaker is currently involved in a word search or engaged in "remembering." These observations are related to word searches and storytelling activities. Further functions mentioned by previous studies include the projection of new topics or thematic transitions and the display of epistemic uncertainty. To date, only speakers' thinking faces have been described, and only few studies have focused on the temporal unfolding of thinking faces in interaction. Addressing this gap, the present study describes how multiple modalities-beyond the face-are temporally coordinated to create multimodal gestalts (Mondada, 2014) of "doing thinking." Furthermore, it investigates thinking displays in children's argumentative activities.

\section{Argumentation as an Embodied Discursive Practice}

In face-to-face interaction, argumentative activities are a multimodal and multiparty field of activity. From the perspective 
of the sociology of knowledge and linguistic anthropology, arguing can be considered a sedimented discursive practice, i.e., as a socioculturally evolved procedural solution for recurrent communicative problems in a speech community (Bergmann and Luckmann, 1995; Hanks, 1996). The communicative problems argumentations are designed to overcome are the interactive management of divergent validity claims as well as the articulation and exploration of (potential) problems (Knoblauch, 1991; Antaki, 1994; Quasthoff et al., 2017; Arendt, 2019). Arguing is thus closely related to the co-construction of knowledge: it can be a vehicle for exploring proposals, negotiating divergent viewpoints, and making joint decisions (e.g., Stevanovic, 2012; Heller, 2018). At the same time, arguing enables participants to constitute social orders and negotiate identities (e.g., Goodwin and Goodwin, 1987; Danby and Theobald, 2014).

Recent research suggests that epistemic stancetaking (e.g., Kärkkäinen, 2006; Heritage, 2012) provides a crucial resource for argumentative activities (Keisanen, 2007; Heller, 2018; Kreuz and Luginbühl, 2020; Morek, 2020). Here, the display of a tentative, uncertain or determined stance is especially consequential not only for shaping local participant frameworks but also for the larger framing of the activity as persuasive and competitive or as exploratory and collaborative (Sterponi, 2009; Ehlich, 2014; Bova and Arcidiacono, 2015; Heller, 2018; Mundwiler and Kreuz, 2018; Hannken-Illjes and Bose, 2019). These frames entail different epistemic orders that differ in the degree to which the jointness of an emerging decision (Stevanovic et al., 2017) is established.

In addition to epistemic stance displays, embodied resources are also vital for decision-making processes. Stevanovic et al. show that body-sway patterns and pitch register provide important resources for interpersonal coordination in joint decision-making. Hannken-Illjes and Bose (2019) show how children use the synchronization of bodily actions and paraverbal resources such as loudness to frame their argumentation as cooperative whereas what they call "agonistic situations" exhibit a rather arhythmical or discontinuous coordination. Manual actions and gestures are other important resources to make "embodied arguments" (Mirivel, 2011). For example, reciprocal palm up open hand gestures (Kendon, 2004; Müller, 2004; Streeck, 2007) serve as publicly visible resources to embody the give-and-take of arguments (Schönfelder and Heller, 2019), thus facilitating the orderly production of contiguous responses. In a similar way, interlocutors use gestures as a device to facilitate understanding by segmenting structural parts of their arguments (Jacquin, 2017). These studies indicate that arguing is an embodied discursive practice. The present paper investigates the thinking face as a potential resource for interpersonal coordination in children's argumentative activities.

In a previous study on embodied resources in children's argumentative decision-making, Heller (2018) examined a sequence in which the thinking face served as a framing device for organizational problems on various interactional planes. First, by projecting that the performer of the thinking face is going to claim the floor, the display facilitates the organization of turn-taking. The publicly visible performance mobilizes the recipientsvisual attention. The latter shift their gaze to the speaker and refrain from taking the turn. Second, by giving the audience a clue to what kind of action the turn will be doing, the thinking display provides an important device for action formation. Together with lexical and morphological resources (e.g., verba dicendi, subjunctive mood), it serves to display a thoughtful stance and thus instructs the recipients to expect the ensuing action to encompass a disclosure of the incipient speaker's thoughts, i.e., a proposal and the "thoughts behind it." Third, with regard to the larger activity, the placement at the beginning of the sequence projects how the speaker conceptualizes the nature of the joint projectargumentative decision-making - as one that involves thorough thought. The thinking face thus also helps to frame the activity as collaborative reasoning ${ }^{1}$. The study indicates that thinking faces can fulfill several functions for the framing of argumentative decision-making and the coordination between the participants. However, it is based on a single instance. The present study therefore examines interactional and epistemic functions of embodied displays of "doing thinking" in a systematic approach.

\section{MATERIALS AND METHODS}

\section{Participants and Data}

The data for this study come from a larger corpus of video recordings of 90 monolingual and multilingual children aged 7 13 years. All children attended inclusive classes in primary and secondary schools, located in different socio-economic milieus in Germany. For the present study, only 10 groups of 32 older children (aged 10-13 years) were selected. Within the school setting, groups of three to five children were video recorded as they dealt with different problems. One of these problems concerned a fictitious shipwreck and required the children to make a joint decision on essential survival items (for a similar setting see Kreuz and Luginbühl, 2020). Another task entailed a moral dilemma that arises in the course of an attempt to cheat in a painting competition. Both problems provoked argumentative activities in the groups. For each task, there was a handout with a graphic illustration on the table. The children sat in a semicircle around the table and pulled the handout toward them, pushed it into the middle and pointed to individual illustrations. To minimize disturbance of the groups, only one camera was used. Because the children sat in a semicircle, occurrences of thinking displays were usually easy to identify, but not always visible in detail. The analysis is therefore limited to children at whom the camera was directed frontally. The video recordings used for the present study comprise $58 \mathrm{~min}$ in total.

\section{Analytical Approach}

Videos were pre-coded for all occurrences of thinking faces. Each instance was coded to indicate whether it was

\footnotetext{
${ }^{1}$ The term "reasoning" is used here synonymously with that of conversational argumentation; "collaborative reasoning" refers to an exploratory mode of jointly developing and weighing arguments.
} 
produced by the speaker or the recipient. After the analysis of different cases, the data were reviewed a second time. This way, a total of 29 thinking displays of speakers and 28 of recipients were identified. All occurrences were analyzed in detail, using a multimodal approach to situated interaction (Goodwin, 2000; Streeck et al., 2011; Heath and Luff, 2013). By examining how participants themselves orient to each other's actions sequentially, the analysis reconstructs how they use and interpret thinking faces and accomplish particular activities. In analyzing the total of 57 occurrences, five practices of embodying "doing thinking" emerged. The following section presents a prototypical example for each practice.

The present paper has two interrelated aims. Given that embodied displays of thinking have received only little empirical attention, the first aim is to describe how multiple modalitiesbeyond the face-are temporally coordinated to create various multimodal gestalts of "doing thinking." A second aim is to generate knowledge about the interactive and epistemic functions of thinking faces in children's argumentative activities.

The detailed description of embodied displays of "doing thinking" tries to reconstruct what is visible to the interlocutor as a whole. Since not only facial expressions but also other modalities are involved, I refer to these displays not as thinking faces, but as embodied displays of "doing thinking." They are best conceived as multimodal gestalts (Mondada, 2014, p. 139), i.e., a "web of resources formatting an action." Accordingly, the analytic orientation is on the multiple modalities that contribute to embodied displays of thinking, e.g., typical body postures, particular gaze practices, silence or specific linguistic resources. Since in the data thinking displays were closely associated with the use of (other) epistemic stance markers, special attention is paid to lexical (e.g., modal adverbs and particles) and morphological (e.g., subjunctive) markers of epistemic modality as well as syntactical formatting (e.g., conditional constructions). The focus is on how these resources are temporally coordinated not only for the publicly visible display of "doing thinking," but also for the organization of turn-taking, the shaping of embodied participation frameworks (Goodwin, 2003), the participants' positionings, and the framing of the larger activity.

The transcription follows the GAT 2 conventions proposed by Selting et al. (2011). The multimodal annotation was adapted from Mondada (2018). The description of facial and other bodily actions is informed by the emic approach and based on easily recognizable colloquial descriptions (Birdwhistell, 1970; Peräkylä and Ruusuvuori, 2006). To represent relevant bodily actions and action components, stills were extracted from the videos and temporally aligned with the emerging verbal utterance. For reasons of anonymization, the stills had to be converted into drawings ${ }^{2}$ that capture the most relevant aspects of bodily behavior. Bold print of single words or syllables marks the exact point in time when the frame grab was taken. Since the analytical focus is not on gesture, I do not

${ }^{2}$ Adobe Photoshop was used to create a sketch effect and to erase or change identifying features. transcribe different gesture phases to ease the readability of the transcript.

\section{ANALYSIS: VARIOUS EMBODIED DISPLAYS OF "DOING THINKING" IN ARGUMENTATIONS}

This section reconstructs different practices of embodying "doing thinking." The thinking displays in the data varied in their multimodal gestalts, their turn position, and their alignment with verbal talk and thus serve different interactive and epistemic functions. Furthermore, both speakers and recipients have produced them. Speakers' practices entail embodiments of (1) envisioning and embodying a hypothetical scenario, (2) thoughtful searching for (a part of) an argument, and (3) presenting a position as well-reasoned and thoughtthrough. Recipients' practices comprise embodiments of (4) co-imagining and exploring a scenario described by the current speaker, and (5) independent and critical thinking. The next section describes speakers' practices of using thinking displays.

\section{Speaker Displays of "Doing Thinking"}

Speaker displays of doing thinking occur both in singleunit and multi-unit turns (i.e., "big packages," cf. Sacks and Jefferson, 1992: II). When they are produced within multiunit turns, they are often part of developing a hypothetical scenario and involve solitary or joint origo displacements (Bühler, [1934] 1999). In these contexts, they serve to establish a more or less shared responsibility for developing an argument. In contrast, their use in single-unit turns such as "simple" proposals or statements of opinion does not involve a displacement of the origo. In these contexts, thinking displays are used to present a position as already being well-reasoned and determined.

\section{Envisioning and Exploring a Hypothetical Scenario}

In multi-unit turns, thinking displays can take the form of an extended performance that accompanies speech. This type of temporal alignment implies that the audience participates in the development of a hypothetical scenario from the outset and that speaker and listener share the epistemic responsibility for its exploration. Extract 1 is taken from a group that consists of four children, Anna, Jona, Marko, and Sara. Only Jona and Sara are involved in the following sequence. Jona and Sara first disagreed on the question as to whether the tent is essential for survival. Then Jona establishes an obligation to provide reasons (Heller, 2014). As part of her reasoning, Sara produces a longer-lasting thinking display.

While Jona challenges Sara's position (line 30: “why?"), he cups his chin in his hand, thus assuming a thinking posture (see below) and conveying a thoughtful stance. Looking at each other, he and Sara establish a facing-formation (henceforth: F-formation, Kendon, 1990), entailing that participants "orient their bodies in such a way that each of them has an easy, direct, and equal 


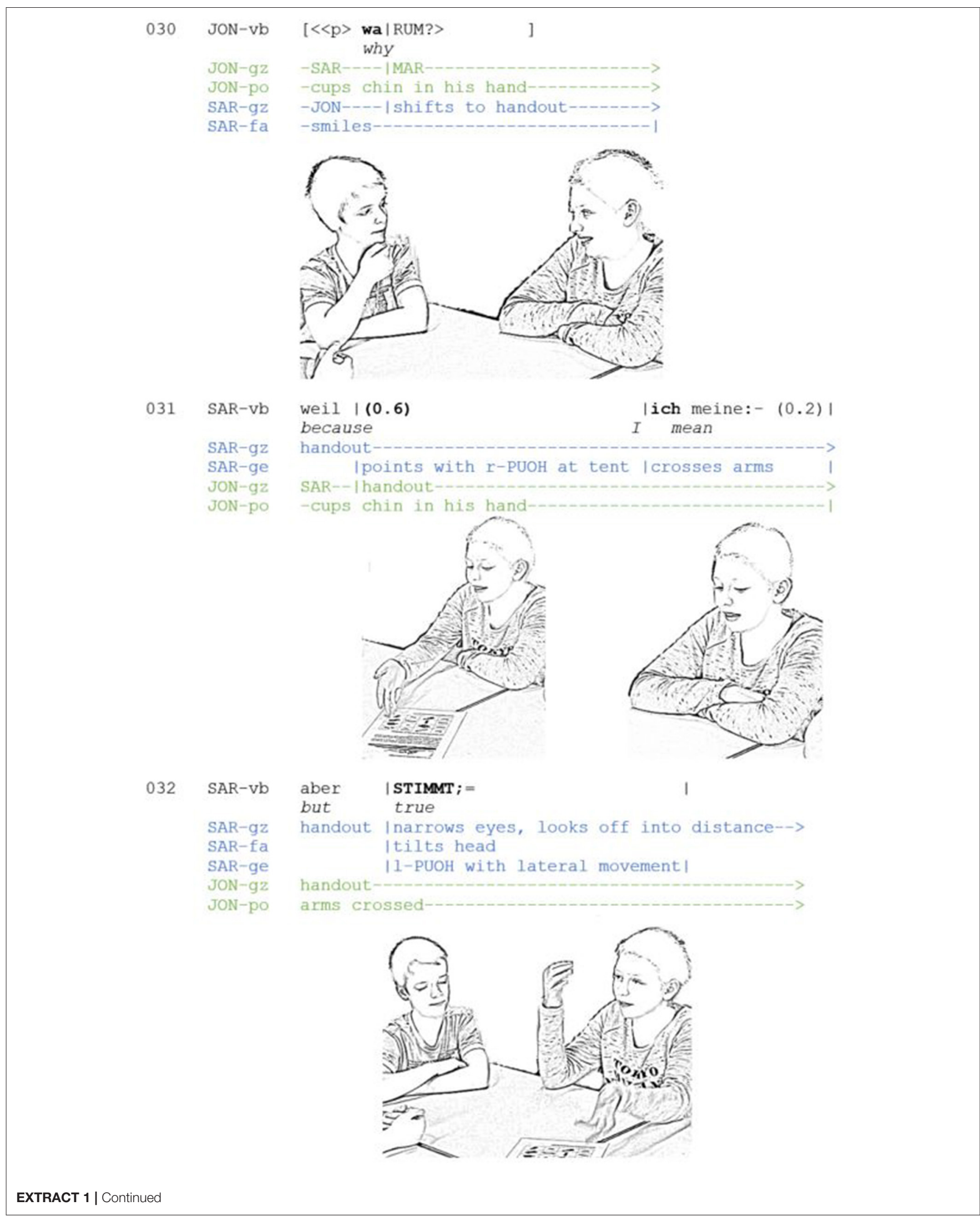




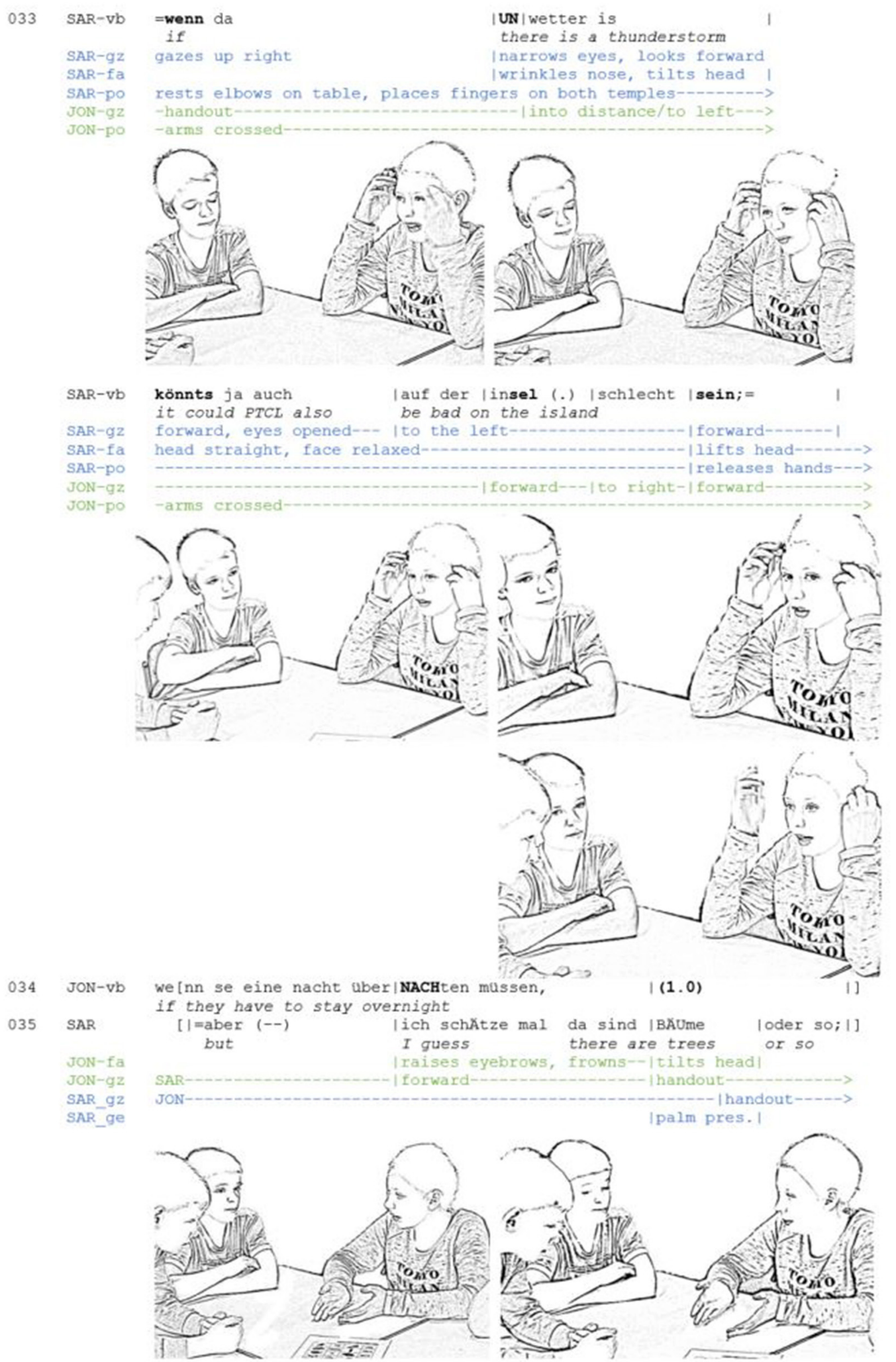

EXTRACT 1 | AE_G10_FB_30-34 (JON: Jona, SAR: Sara). 
access to each other's transactional segment" (including mutual gaze; Kendon, 1990, p. 239). When Sara begins to provide a reason, her pointing to the handout also shows that she is engaged with the objects and participants in the here-and-now (line 31). Overlapping with "I mean:-" she crosses her arms and leans back, thus gradually moving out of the F-formation. After that, she abandons her justification and concedes "but true." At the same time she tilts her head, looks off into the distance, with her eyes narrowed and moves her open hand sidewards ("palm lateral," cf. Kendon, 2004, p. 275). Note that she changes from the right to the left hand. According to Müller (2004, p. 249), such antagonistic lateral movements of the open hand add the idea of "cutting" to the core meaning of the so-called "palm up open hand gestures" (cf. Kendon, 2004; Streeck, 2007). Here, the gesture conveys that the speaker's original position is no longer pursued. Together with the other resources, the gesture thus indexes a change of mind. This embodied change of mind serves as a transition into the display of "doing thinking." Resting the right elbow on the table, the speaker moves into an inflexible body posture.

The thinking face is produced in the context of a hypothetical scenario, which serves to explore the implications of a proposed action. The following resources are progressively assembled and combined to embody the process of envisioning and exploring a hypothetical scenario: first, the conditional "if" is temporally coordinated with another change in the bodily posture. The speaker rests the second elbow on the table and places her fingers on her temples, assuming an inflexible posture. In this posture, head, and hands are fixed, signaling that neither gestures nor head movements are to be expected for the duration of this bodily configuration. Furthermore, the posture entails that the speaker touches herself. As opposed to social touch that allows us to engage with others (cf. Goodwin, 2017), self-touch implies that the individual gets entangled in the haptic-kinetic perception of her own body and shields herself from other stimuli. This kind of posture can be considered stylized; similar postures are known, for instance, in arts (e.g., "The Thinker" by Rodin) and are called thinking posture here. Assuming this posture, the speaker creates a marked contrast between speaking and silence as well as movement and immobility, indicating that she is temporarily absorbed by her own thoughts. She thus brings about a change in her involvement with others (Kendon, 1990, p. 187). The posture is combined with looking up into an "empty space," as if the idea were to be found on the ceiling (cf. Ekman, 1979, p. 186). This gaze withdrawal has the result that the F-formation is temporarily suspended. Instead, the speaker embodies a change in the direction of her attention: she indicates to her co-participants that she no longer perceives the external surroundings, but rather directs her attention inwards, toward a world of thought, in which she first needs to make up her mind before she can share her idea with her co-participants. Since the gaze is not focused on objects or persons in the here-and-now but instead on entities that only exist in the speaker's mind, I refer to this gaze behavior as imaginative gaze. The latter is interpreted by the participants with respect to the ongoing activity.
This implies that this gaze practice assumes an interactional function (Rossano, 2012, 2013) and informs the participants' mutual understanding.

In her description of the scenario, Sara demarcates condition and consequence through observable changes in the emerging multimodal gestalt. The condition "there is a thunderstorm" (line 33) is accompanied by a display that conveys that the speaker is envisioning the scene in the moment of her own description. The head is tilted, the nose wrinkled and the eyes are narrowed and looking forward. In this way, the process of "zooming in" or "focusing on" a virtual image that gradually emerges before her mind's eye is embodied. In contrast, the hypothetical consequence "it could PTCL also be bad on the island" is temporally aligned with a relaxation of the face. At the same time, the head returns to an upright position; the eyes are opened and wandering to the left, conveying the impression that the scene is now clearly visible and explored in more detail. On the verbal level, the untranslatable German modal particle "ja" implies that what is just being said can be assumed to be shared knowledge (Reineke, 2016, p. 96). Altogether, the alignment and juxtaposition of these resources embody a change of state (Heritage, 1984) from an incomplete to a complete image, from an uncertain to a certain stance. While the thinking posture with the facial self-touch remains stable and thus functions as a bracket (Scheflen, 1965) for the protasis and apodosis, the changes in the facial expression and the wandering eyes embody two processes, envisioning and exploring a scenario. The whole process is completed when the speaker reaches the turn-final word "sein." In this moment, she lets go of her temples and shifts her gaze to the front.

I would like to argue that the thinking posture and the imaginative gaze serve to indicate that the current speaker is "stepping out" of the here-and-now and displaces her origo, i.e., the "here-now-I system of subjective orientation" (Bühler, [1934] 1999, p. 117), to an imaginary space. It is important to note that this displacement is not accomplished privately but as a publicly visible performance. The temporal organization of the multimodal resources ensures that the recipients can likewise displace their origo. Through the simultaneity of verbal description and embodied imagination, they are enabled to jointly imagine the hypothetical scene. This can be clearly evidenced by the recipient's behavior: resting his crossed arms on the table, Jona signals that he assumes the role of the listener and will not interfere (line 32). When Sara begins to sketch the condition ("thunderstorm"), he lifts his gaze from the handout and starts to look into the distance. While Sara goes on to draw a consequence, he first continues to look to the empty space in front of him and then shifts his gaze to the right and back (line 33), conveying that he, just like Sara, is envisioning and considering the scenario from different sides. By refraining from looking at Sara, he demonstrates that he is not simply agreeing with her argument but responsibly exploring the scenario on his own. The recipient's reciprocation of the thinking face shows that the participants have established a participation framework for 
joint imagination (Stukenbrock, 2017; Heller, 2019; Kinalzik and Heller, 2020).

In this way, the argumentation is staged as not yet completed or unchangeable, but as being in the process of formation. Furthermore, the speaker enables the recipients to participate in "the gradual production of thoughts whilst speaking" (Kleist, 1805 ) and therefore invites them to explore the imagined scenario together with her. As fellow thinkers they share the epistemic responsibility (Stivers et al., 2011) for examining a hypothetical scenario. This is also underlined by the fact that Jona elaborates the scenario in his next turn: "if they have to stay overnight" (line 34). This co-construction (Kreuz and Luginbühl, 2020) further supports the reasoning and turns it into a shared argument. In comparison to the second example, the speech-accompanying embodiment of thinking has the effect that the listeners are involved in the process of imagination from the beginning and thus participate more intensely in developing the argument.

\section{Thoughtful Searching for (a Part of) an Argument}

In the data, thinking displays also occur when the speaker is searching for (a part of) an argument. In these cases, the thinking displays only last for the duration of the search. They also differ in terms of their multimodal gestalts and their coordination with speech from the displays described in the previous section.

Extract $\mathbf{2}$ is taken from a discussion about the moral dilemma. While Jona suggested that Tom should only talk to his parents about Marie's cheating (Tom and Marie both belong to a fictional scenario) at the painting contest and leave it up to them to decide whether to inform the teacher, Sara proposed that Tom himself should take action on the cheater. After Jona has abandoned his original position and supported Sara’s proposal, Sara develops a justification for the now shared position. In the course of formulating her justification, Sara hesitates and engages in an embodied search for the second part of her argument.

Sara projects her argumentation through verbal and bodily means. The causal connective because is temporally aligned with moving the open hand palm up toward the table. By extending the open hand into the participants' interactional space the speaker enacts the idea of "giving" or presenting an "abstract discursive object" (Müller, 2004, p. 233; also cf. Kendon, 2004, p. 264) - here: a reason -, and offers it for joint inspection. Placed at the beginning of a new unit of her multi-unit turn, the combined resources thus foreshadow the pragmatic function (cf. Streeck, 2009 , p. 171) of "providing a reason." In the present case, the reason includes a hypothetical scenario, which is marked by the conditional "if" (line 68) and the subjunctive. In this way, the speaker not only projects the type of action but also indicates her epistemic stance: what she is going to say has a hypothetical and tentative status. Sara uses the hypothetical scenario to play through the consequences of her proposal (getting Marie to confess her deceit). As she formulates the protasis ("if she would admit it herself,"), her gaze is focused on the handout, and with a pointing gesture, she disambiguates the reference of "she." The recipient, Jona, follows her gesture. Thus, both participants' visual attention is oriented toward an object in their immediate here-and-now (Bühler, [1934] 1999). After the condition has been verbalized, two pauses and the lengthened "then:" indicate a hesitation. In the ensuing pause, Sara withdraws her gaze from the handout and shifts it to an empty space. Such gaze withdrawal while searching for a word has frequently been noted (Kendon, 1967; p. 38; Goodwin and Goodwin, 1986; Goodwin, 1987; Weiß and Auer, 2016). In addition to the imaginative gaze, Sara also retracts her arm and supports her head in her hands. This thinking posture emphasizes that the speaker is temporarily absorbed by her own thoughts or temporarily "away" (Goffman, 1963, p. 69)—engaged in solitary thinking and not ready to interact. In this way, it also serves to create an embodied participation framework for "forming an opinion before speaking."

Decisive for the embodiment of searching for (a part of) an argument is the dynamic of the inwardly directed gaze, which is particularly prominent against the background of the immobility of the body. At first, the gaze is directed forward, then moved to the left and finally downwards, with the eyes almost closed. The eye movements are temporally coordinated with verbal resources or with their absence. The gaze forward coincides with the first pause; the wandering of the eyes to the right is aligned with "then:" i.e., with the verbal resource prefacing the consequence or apodosis. The downward oriented gaze, which also concludes the eye wandering, occurs in the second pause. This temporally aligned and dynamical wandering of the gaze embodies that a thinking process is currently taking place, involving that the speaker "sees" something with her mind's eye. While the forward gaze creates the impression that the speaker is searching for or developing an idea, the wandering of the eyes to the left, temporally coordinated with "then," conveys that the idea is being advanced. The downward gaze, toward the handout on the table, where the eye movement halts, embodies that the line of thought is now so far developed that it can be shared with the co-participants. It also marks a transition, indicating that the attention is now turned away from the inner world and directed toward the world of discourse. At this moment, Sara releases her posture and performs another forward-gesturing palm presentation gesture to signal that the second part of her scenario, the consequence, is now going to be formulated. Simultaneously, she reorients her gaze to the co-participants and objects within the here-and-now.

Like the embodied envisioning and exploring of a hypothetical scenario, the thoughtful search involves an origo displacement. However, the displacement differs in one important point. Like in Extract 1, the freezing of bodily movements and the reorientation of the gaze serve to indicate that the current speaker is "stepping out" of the here-and-now and temporarily directs her attention to a world of thought that is only accessible to her. The publicly visible performance of "doing thinking," embodied through the dynamically wandering gaze, instructs the recipients to interpret the speaker's displacement as a phenomenon that is nevertheless related to the current argumentative activity and therefore prevents them from taking the turn. In contrast to Extract 1, the speaker first performs a solitary displacement. The placement of the thinking display during the pauses (line 69) ensures that initially only she alone is able to "see" the imagined consequences. Only when she verbally formulates the apodosis, the recipients are enabled to co-imagine the scenario. By granting 


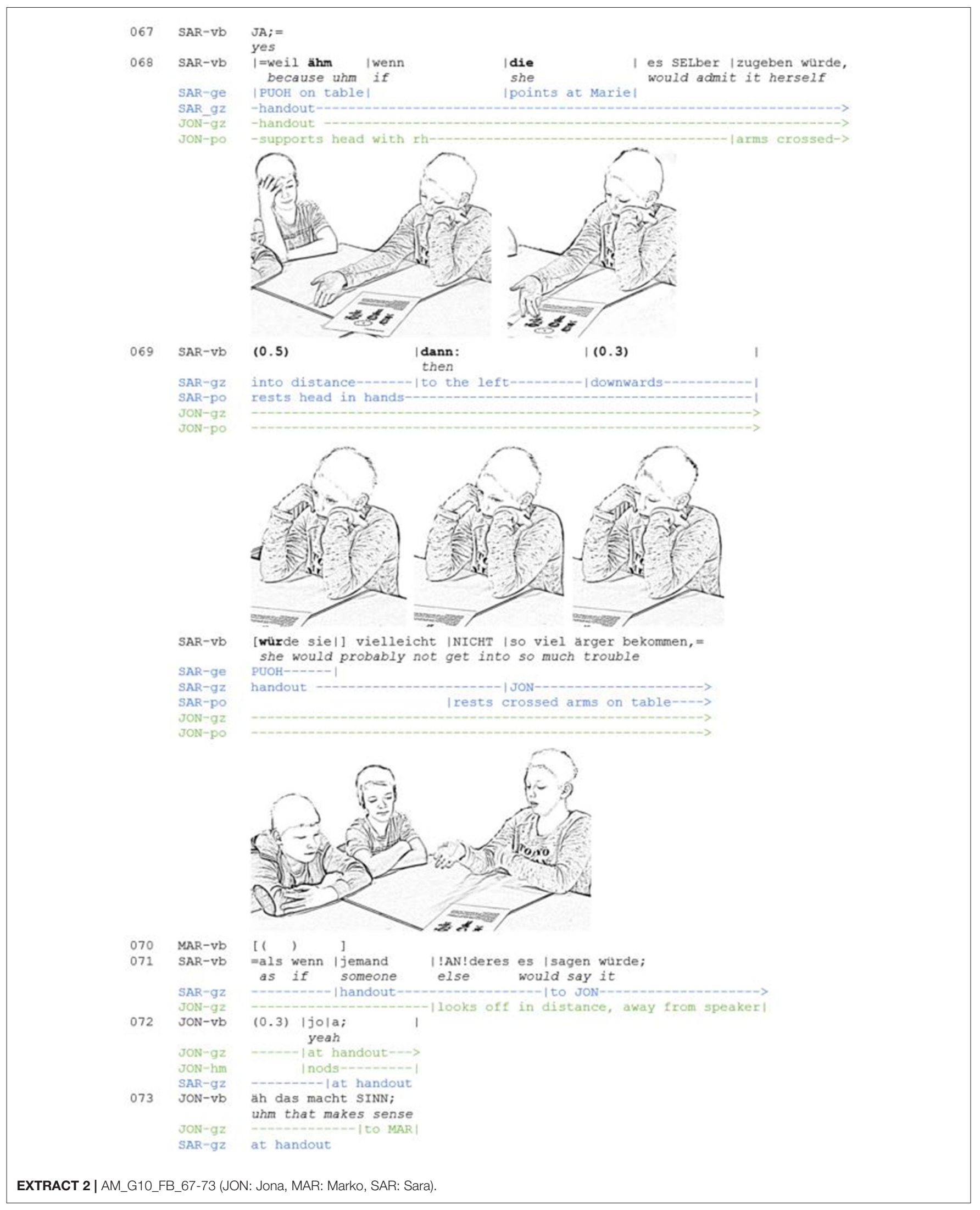


the recipients only delayed epistemic access, the speaker presents the consequence she has drawn as independently tested and weighed, thus positioning herself as a responsible thinker. At the same time, the display of uncertainty (the adverb "perhaps" in the apodosis) conveys that the co-participants have the right and responsibility to be involved in the decision-making process. In this way, the speaker balances the relationship between individual and shared epistemic responsibility. It can be observed that the recipients align with the speaker's embodied search: only when the argument is completed, Jona produces a thinking face himself (line 72), and then agrees with the argument (line 73).

In summary, it can be said that the thinking face here fulfills a similar function as in the word searches examined by Goodwin and Goodwin (1986). The main difference is that the search does not only cover one word, but a component of an argument. The fact that embodied searches are also successful in argumentative activities is remarkable, since proponents and opponents often compete for the turn. The framing of the argumentation and the sequential position in which the embodied search occurs is revealing in this respect. Sara initiates the search for a not yet available reason after an agreement has already been reached. Furthermore, for the most part, the argumentative activity is not framed as competitive but as collaborative (Heller, 2018). The search for a justification that is now taking place is not only made possible by this framing, but at the same time reflexively maintains it

\section{Presenting One's Position as Well-Reasoned and Thought-Through}

A number of thinking displays are found in rather short turns, in which the speakers present their position without further supporting it. In these cases, embodied displays serve to present one's position as well-reasoned and already thought-through. Extract 3 represents the very beginning of the argumentative activity. After Sara has initiated the discussion (line 24: "so"), all parties involved allow a pause to arise (line 25), before Jona takes a stand and produces an accompanying thinking display (line 26). In contrast to the first two examples, his display does not evoke an ongoing thinking process but rather indicates that the process is already completed.

Jona does not simply state his position, but projects it metadiscursively with an epistemic preface (Heller, 2018), using a verbum dicendi and the subjunctive ("I would say"). The preface serves to explicate the pragmatic function of "taking a position" and frames the contribution as a proposal. This means that the proposed decision is presented as one conceivable among possible others and thus as contingent upon the recipients' approval (Sidnell, 2012; Stevanovic, 2012). However, this tentative stance is modified by further epistemic markings in the course of the utterance (see below).

Temporally aligned with the preface, the speaker adopts an inflexible body posture. He rests his elbows on the table and cups his chin in his hands. At the same time his gaze is drawn away from the persons in his immediate interactional space and shifted toward the handout. Additionally, he starts to stroke his chin. Again, this form of self-touch can be considered to be a stylized thinking posture. Against the background of the inflexible body, the self-directed movement serves to mobilize the co-participants' (visual) attention, as can be seen in Sara's reaction: she shifts her gaze to Jona (line 26). Note that in this example the speaker does not shift his gaze while speaking (as in Extract 1). By keeping his visual focus on the handout, the speaker indicates that he is not displacing his origo to an imagined scene in order search or develop an argument. Instead, the constant focus of the eyes contributes to the impression that the speaker has already reached a decision. This is consequential for the epistemic order and will prove to be a major difference to the previous two examples.

In addition, the sequential placement of the thinking display is important: by adopting the thinking posture in turn-initial position and maintaining it throughout the turn's production, the speaker conveys that his or her position has already been thoroughly thought out and does not require further elaboration by the other participants. The speaker thus asserts epistemic primacy (Heritage, 2012). This is also emphasized through the epistemic idiom "in any case" and the nodding which concludes the embodied display. Both resources are used to express epistemic certainty and present the proposal as well-founded and not requiring further justification. By making only an agreement of the other participants relevant, they steer into closing the discussion of the item in question. In their subsequent turns, the co-participants refer directly to the position expressed by Jona. While Marc agrees with the opinion (line 27), Sara establishes a playful dissent by means of a format-tied response (Goodwin and Goodwin, 1987), which is accompanied by smile and mutual gaze (line 28). This friendly challenge to the speaker's pre-determined stance (Heller, 2018, p. 285) shows that such embodied epistemic positionings are not necessarily "successful" and may result in dissent and a rearrangement of the epistemic order.

To summarize, the thinking posture is adopted at the beginning of the turn and maintained for the time of its production. The multimodal gestalt of the display is mainly based on the thinking posture, steady gaze to the handout and the nodding of the head. Verbal resources entail an epistemic preface and markers of epistemic certainty. All these resources serve to present a position as already thought-through. The speaker indicates that he is no longer in the process of searching or developing ideas but has already come to a decision.

The embodied displays described in this section typically occur in short argumentative turns, in which a position is stated without further argumentative support. In contrast to the first two examples, the thinking displays occur in single-unit turns and are temporally organized in ways that do not involve the recipients in the development of an argument.

\section{Summary}

The analyses showed that "doing thinking" was organized as a public practice: the embodied displays of "doing thinking" involve multiple modalities. The components of the different multimodal gestalts are summarized in Table $\mathbf{1}$.

Each of the multimodal gestalts entailed a stylized thinking posture. Although they took different forms-supporting the head or chin in the hand, grasping the temples-, they had in common that the body assumed a rather inflexible posture, while 


$\begin{array}{ll}024 & \text { SAR-vb } \\ & \text { SAR-gz } \\ & \text { JON-gz } \\ 025 & \\ 026 & \text { JON-vb } \\ & \\ & \text { JON-po } \\ & \text { JON-ge } \\ & \text { JON-hm } \\ & \text { JON-gz } \\ \text { SAR-gz } \\ \text { SAR-pO }\end{array}$

Al | so:;

so

handout------>

SAR | handout $->$

$(0.5)$

ich würd

I would

sagen auf JEden |fall

say in any case

|das | ZELT;

rests elbows on table--

strokes his chin-

the tent
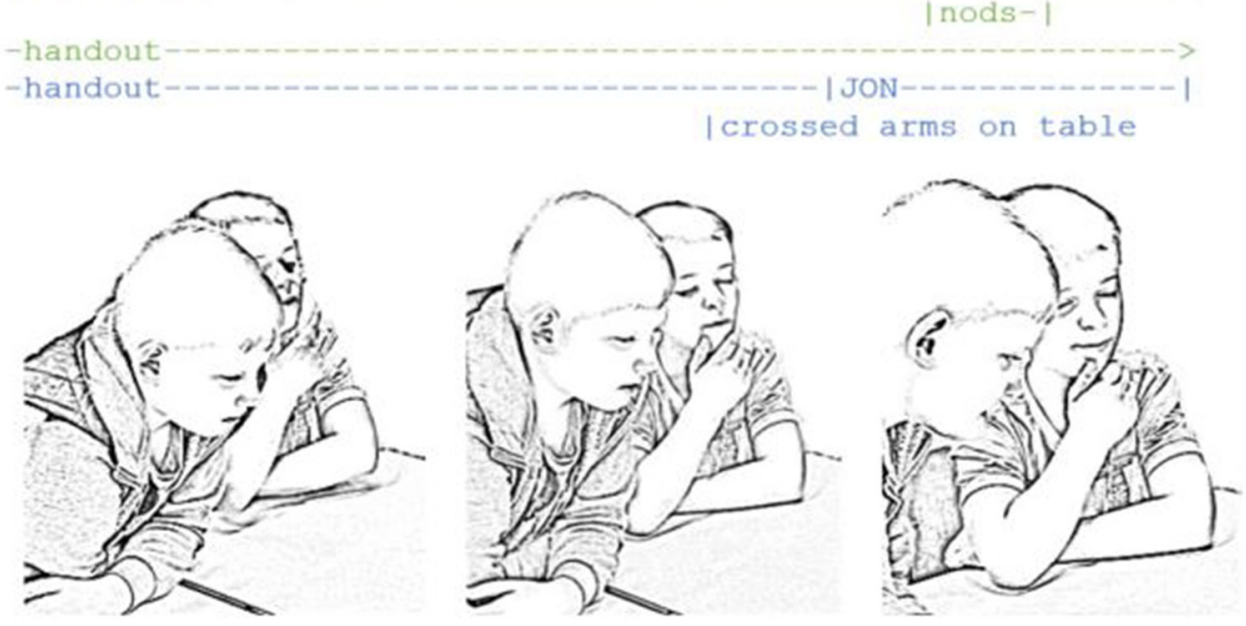

027

MAR-vb

$[<<\mathrm{p}>$ das ZELT; $>$ ]

the tent

MAR-fa

028

$S A R-v b$

nods-----------1

[ich wurd sagen auf] |KEInen

I would say

in no
SAR-gz

SAR-fa

SAR-po

JON-gz

JON-po

handout

handou to

cups chin in hand
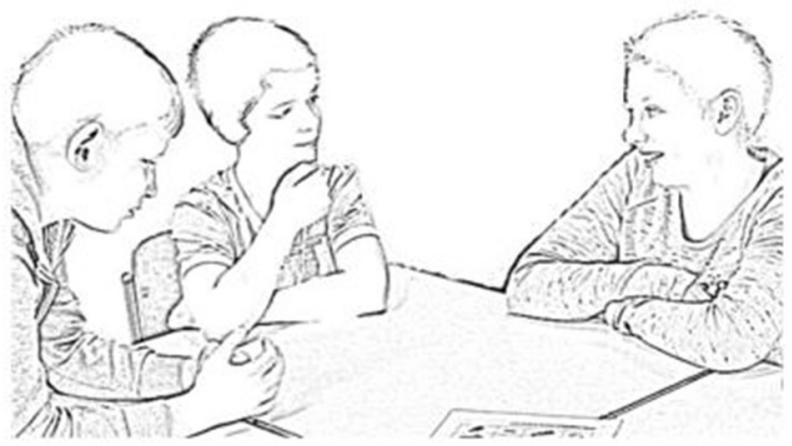

029 MAR-vb

MAR - gz

MAR-hm $[<\langle\mathrm{p}\rangle$ doch $\mid$ das ZELT; $>]$

-handout----_-------->

nods----- 1

EXTRACT 3 | AE_G10_FB_24-28 (JON: Jonah, MAR: Marko, SAR: Sara). 
TABLE 1 | Speakers' embodied displays of "doing thinking."

\begin{tabular}{|c|c|c|c|c|}
\hline $\begin{array}{l}\text { Envisioning and exploring } \\
\text { a hypothetical scenario }\end{array}$ & thinking posture & $\begin{array}{l}\text { imaginative gaze and } \\
\text { focusing, wandering of the } \\
\text { eyes }\end{array}$ & $\begin{array}{l}\text { accompanying speech (in a } \\
\text { multi-unit turn that develops } \\
\text { a hypothetical scenario) }\end{array}$ & $\begin{array}{l}\text { joint imagination, shared } \\
\text { epistemic responsibility }\end{array}$ \\
\hline $\begin{array}{l}\text { Thoughtful searching for } \\
\text { (a part of) an argument }\end{array}$ & thinking posture & $\begin{array}{l}\text { imaginative gaze and } \\
\text { wandering of the eyes }\end{array}$ & $\begin{array}{l}\text { during hesitation (in a } \\
\text { multi-unit turn that develops } \\
\text { a hypothetical scenario) }\end{array}$ & $\begin{array}{l}\text { first solitary, then joint } \\
\text { imagination, independent } \\
\text { and shared epistemic } \\
\text { responsibility }\end{array}$ \\
\hline $\begin{array}{l}\text { Presenting one's position } \\
\text { as well-reasoned and } \\
\text { thought-through }\end{array}$ & thinking posture & steady gaze to the handout & $\begin{array}{l}\text { accompanying speech (in a } \\
\text { single-unit turn in which a } \\
\text { position is stated) }\end{array}$ & $\begin{array}{l}\text { no } \\
\text { displacement/imagination, } \\
\text { epistemic primacy }\end{array}$ \\
\hline
\end{tabular}

the eyes moved vividly. The analysis of the entire corpus shows that the type of posture is not decisive for the functions the thinking display fulfills. The functions rather depend on the activity of the eyes, the coordination with speech or silence and the sequential placement.

Two displays were characterized by imaginative gaze and eye movements that evoked a progress in thought. When imaginative gaze was coordinated with silence, it enacted a solitary displacement; when it accompanied the verbal description of the scenario, it had the effect of involving the listeners in the process of imagination. The absence of the imaginative gaze served to convey that the speaker was no longer in the process of searching or developing ideas but had already come to a decision. The different uses of thinking displays were consequential for the epistemic ecology of the activity in that they constituted an independent or shared epistemic responsibility for argumentative decision-making.

\section{Recipient Displays of "Doing Thinking"}

This section examines recipient displays of "doing thinking" in multi-unit turns. With regard to displays of emotion, Kaukomaa et al. (2015) have demonstrated that the recipients' facial expressions not only display understanding of what is said but may perform systematic operations on the speaker's turn and the emerging activity. In the present data, recipients use thinking displays to demonstrate their alignment with the ongoing activity. In addition, they signal either agreement or disagreement, or an exploratory or critical stance. In this way, they provide an ongoing feedback not only for the current speaker, but also for the other co-participants. Thus, displays of "doing thinking" are a resource to shape the emerging participation framework while listening.

\section{Embodying Independent and Critical Thinking}

In the data, recipients were found to use embodied thinking displays to embody a critical stance and project that they are going to claim the floor. Extract 4 stems from a group of five children; only three of them are involved in the following sequence: Deana, Yeliz, and Zarif. Several proposals are made on how to deal with the cheating in the painting competition. When
Deana makes an alternative proposal, Zarif visually displays that he takes an independent and critical stance on it.

Shortly after Deana has projected an alternative proposal (line 145), Zarif agrees with a suggestion made by another participant (line 146). When Deana goes on to formulate her idea (line 147) and arrives at the semantic core element ("new picture"), Zarif does multiple things at once: he assumes a thinking posture, with his elbows rested on the table and his head supported by his right hand. Additionally, he frowns. Together with the thinking posture, the frown serves as a resource for the recipient to mark an element of the speaker's utterance as problematic (for speaker frowns see Kaukomaa et al., 2014). Looking at Deana, who shortly afterwards establishes mutual gaze, Zarif checks whether Deana notices his display. Establishing mutual gaze with Zarif while speaking, Deana is in fact able to perceive visually that Zarif is not only listening carefully, but also displaying a critical stance toward her proposal. At the end of the unit of the multi-unit turn, both participants dissolve eye contact. By withdrawing his gaze from the objects and participants in the interactional space and looking off into the distance, Zarif demonstrates to the other parties that he thinks independently about the proposal (Stevanovic, 2012) and forms his own opinion. In this way, he positions himself as a responsible and critical thinker. At the same time, Zarif demonstrates that he listens to Deana attentively: as the latter continues her turn (line 148) and disambiguates the reference with deictic gestures to the fictitious protagonists, Zarif's gaze follows Deana's hand. Toward the end of the turn, i.e., at the transition place, Deana and Zarif again establish mutual gaze. In this moment of mutual perception, Zarif frowns again and also presses his lips together, thus indicating stronger doubt. Simultaneously, Deana produces a two-handed palm lateral gesture, with which she declares the discursive object to be obvious (Kendon, 2004, p. 275f.; Müller, 2004, p. 243f.). Without speaking, Zarif has thus projected a dissent.

In the ensuing pause (line 149), Zarif transforms the frown into a prolonged display of "doing thinking" with which he projects, among other things, that he going to take the turn. The trajectory of facial expressions thus enables a smooth transition from the role of the recipient to that of the speaker. Furthermore, they serve as a pre-element for the ensuing disagreement (Pomerantz, 1984) and thus project that type of next action is to be expected. Maintaining the thinking posture, Zarif looks off in the distance, thus indicating a change in the direction 


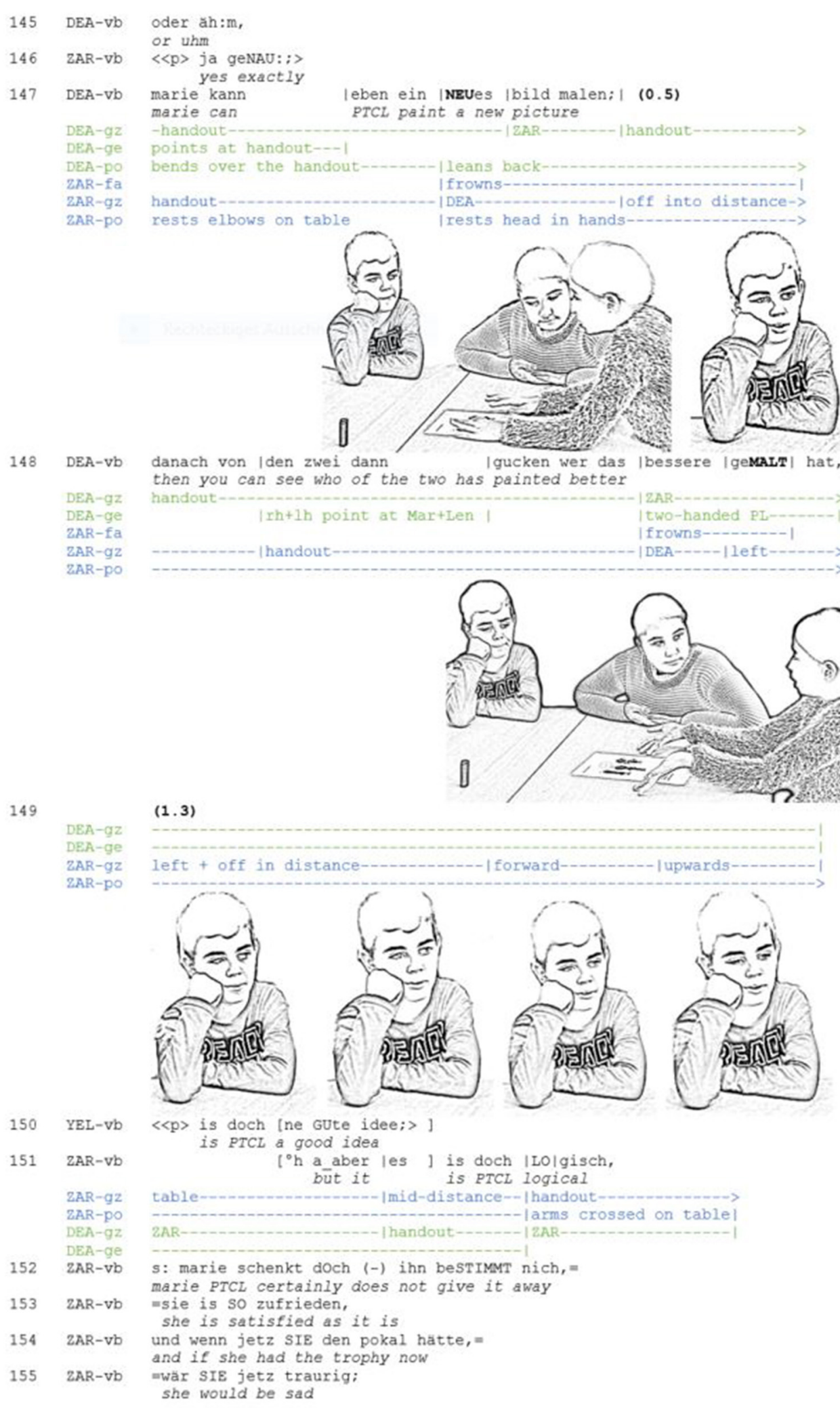

EXTRACT 4 | AM_G11_S_145-155 (DEA: Deana, YEL: Yeliz, ZAR: Zarif). 
of his attention. Then his imaginative gaze describes a circle. The thinking posture and the wandering of the eyes yield a multimodal gestalt that embodies the recipient's displacement to the scene previously described by the speaker. In contrast to the next example, the embodied displacement is solely performed by the recipient. Initiating the embodied display only at the end of the speaker's turn and maintaining it throughout the pause, the speaker demonstrates that he is currently engaged in independent and critical thinking about the speaker's proposal.

Together with the succession of frowns the embodied display of independent thinking that accompanies the speaker's turn serves a number of functions on multiple interactional planes: first, it provides an online-feedback both for the current speaker and for the other co-participants on how the listener received the current speaker's argument. The publicly observable formation of the recipient's epistemic stance enables the other recipients to assess even during turn production how the perspectives of the individual participants, including their own, relate to each other. Obviously, this facilitates the coordination between the participants. With regard to the organization of turn-taking, the embodied display projects that the recipient is going to claim the floor. This is in line with Kendon's (1973) observation with regard to what he calls "long utterances" [e.g., "when people are exchanging points of view, (...) or exploring one another's knowledge of something," p. 61]. Before the expectable end of the current speaker's turn, recipients regularly look away in order to signal readiness to take the turn. Producing thinking displays at turn-final position is thus a practice of self-selection (Sacks et al., 1974), which may be especially functional in multiparty conversations. In addition, the embodied display of critical thinking also prefigures what type of next action is to be expected: a multi-unit turn with which the plausibility of the speaker's position is challenged (line 151-155). It is thus a resource for the recipient to shape the emerging participation framework and to position himself as an attentive and aligning, yet autonomous thinker.

The data show that embodied displays of independent and critical thinking can also cause the current speaker to change his or her epistemic stance in the course of utterance production (cf. Kaukomaa et al., 2015 for emotional stances). This underlines the fact that recipient displays fulfill essential functions for the coordination between the participants.

\section{Co-imagining and Co-exploring a Scenario Described by the Current Speaker}

In contrast to the previous example, recipients may also embody that they co-imagine a scenario described by the current speaker and agree with his or her conclusion. These displays accompany the speaker's multi-unit turn. The following and final Extract 5 again stems from a discussion about the moral dilemma. Zarif suggested to return the trophy to the teacher and to organize a new competition (line 126-127), thus constructing a new scenario. When Yeliz expands Zarif's proposal, he accompanies her multi-unit turn with an embodied display of "doing thinking."

After Deana has agreed with Zarif's proposal, Yeliz produces a multi-unit turn (prefaced with "or," line 129 and 130) in which she co-constructs but also slightly modifies Zarif's proposal. She first reformulates the first part of the scenario (line 130) and then adds a new idea to it: the teacher should award two trophies (line 131), with the consequence that both children win the competition (line 132). In this way, Yeliz makes the proposal a shared one. While she elaborates the scenario, Zarif reciprocates her posture and gaze behavior. First, Yeliz and Zarif adopt reciprocal postures: they lay their arms on the table and none of them reaches for the handout. Thus, both of them assume a rather inflexible posture. Likewise, both of them engage in imaginative gaze. While Yeliz formulates her proposal, she gazes in the direction of the handout. Yet she does not seem to focus something specific but rather gazes into the void, thus indicating a change in the direction of her (visual) attention. Zarif first shifts his gaze to the left, then in the empty space in front of him. This multimodal gestalt embodies that the scenario described by the current speaker gradually emerges before his mind's eye. Subsequently, his embodied display of co-imagining and co-exploring a scenario changes dynamically; each change is aligned with one element the semantic-pragmatic structure of the speaker's multi-unit turn. At end of the second turnconstructional unit, when Yeliz formulates the semantic core element of her alternative ("another/a second trophy"), Zarif lifts his head and shifts his gaze upwards, thus enacting that he has taken a new step in thought. In this way, he provides the other participants with a visible clue that he is following the speaker's idea step-by-step. In fact, this clue does not go unnoticed: Deana shifts her gaze toward Zarif (line 131). When Yeliz draws the consequence (line 132), Zarif raises his gaze again and lets it wander first to the left. Overlapping with the final element "both [...] a trophy," he looks forward again and also opens his eyes and mouth - a multimodal gestalt that Heath et al. (2012, p. 217) refer to as "surprised mouth" -, giving the impression of having come to an insight. This is followed by a "个YE:S" which is lengthened and also produced with a small pitch upstep. The prosodic design results in the fact that the "yes" is not only heard as a confirmation, but as an indication of an insight or "aha" moment. Together with the "yes" the facial expression thus embodies a change of state (cf. Mondada, 2011). Similar to "oh," which is produced as a response to information of some kind and enacts a change in its producer's state of knowledge (Heritage, 1984), the embodied change of state described here serves not only to accept the prior talk as informative but also to register that the proposal developed by the speaker was persuasive. This way, it displays both a change of state and a change of stance.

Until now, the participants have not established mutual gaze. Instead, the recipient's ongoing and vivid wandering of the eyes served as an embodied display of co-imagining and coexploring the scenario that the speaker currently describes. The participants have thus created a participation framework for joint imagination, within which each participant envisions and inspects the scenario. Only after the scenario was concluded and Zarif indicated a change of state and epistemic stance, the participants establish an F-formation: Zarif initiates mutual gaze with Yeliz, while Deana also looks at Zarif. Overlapping with Deana, Zarif then begins to elaborate Yeliz' idea by explicating the consequence in more detail. His palm presentation gesture 


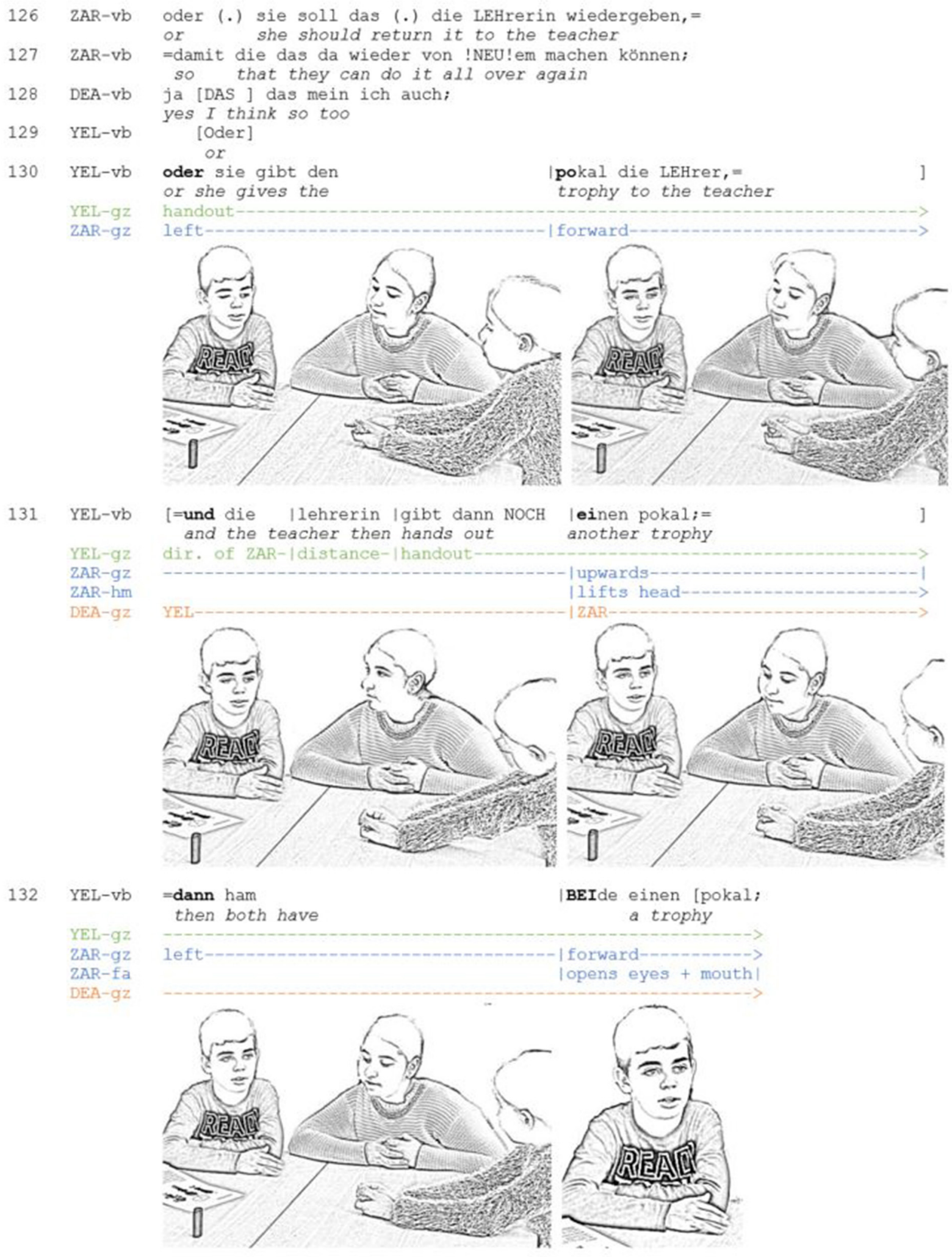

EXTRACT 5 | Continued 


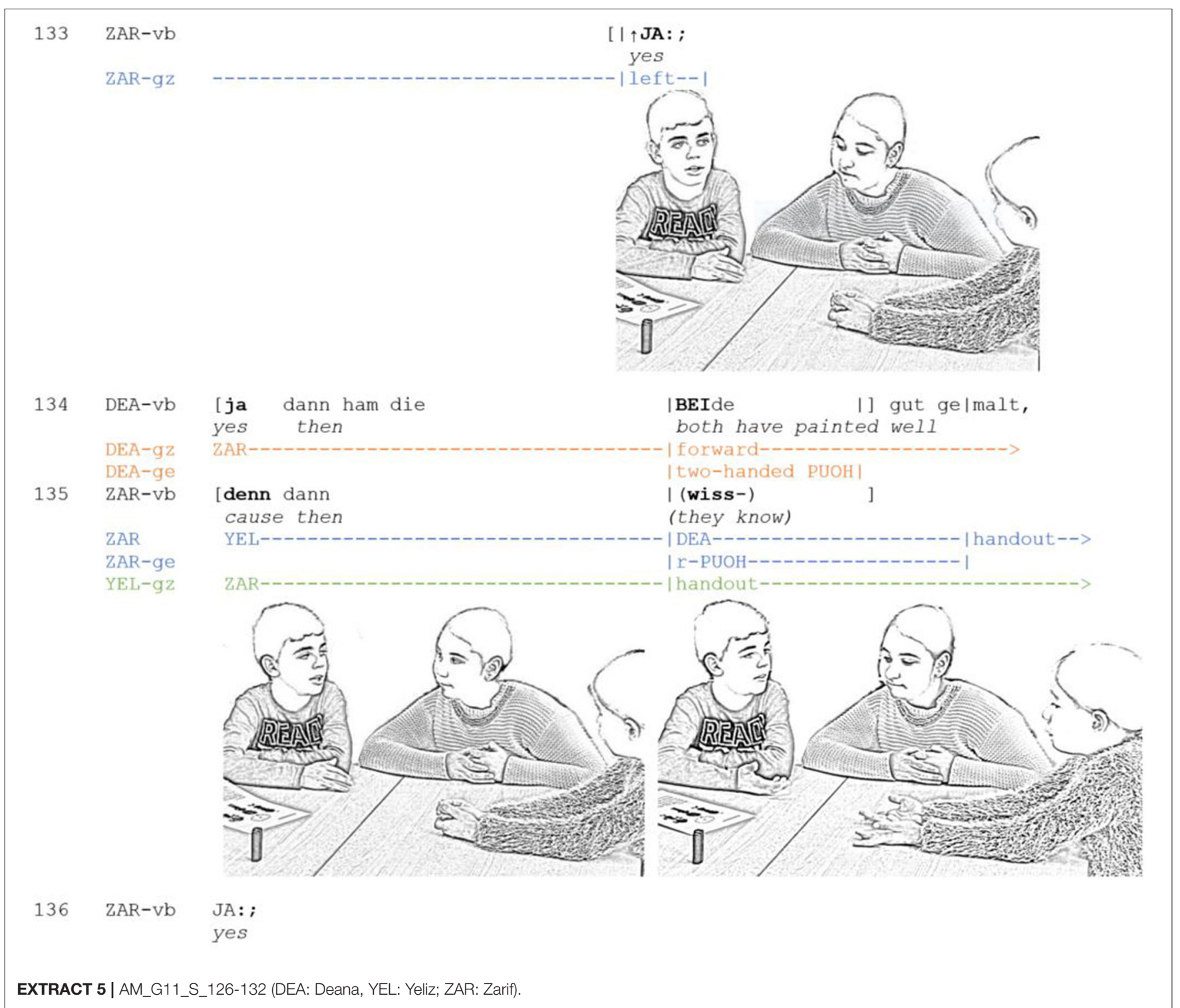

(line 135) is produced in concert with Deana's two-handed palm presentation gesture. Both gestures serve to project a conclusion or concluding comment on the prior proposal (cf. Kendon, 2004, p. 270). By temporally aligning their gestures and directing them at each other, both participants mutually demonstrate to each other that they have reached a similar conclusion at the same time (Schönfelder and Heller, 2019). Although Zarif abandons his turn, it is clearly visible that both turns were designed to further co-construct the shared argument. The sequence is thus framed as a collaborative reasoning, in which hypothetical scenarios are jointly explored.

To summarize, within this process of co-constructing an argument, constant wandering of the eye is used by the recipient to demonstrate that he co-imagines the scenario described by the current speaker. Avoiding eye contact is important to convey that the recipient first envisions and explores the scenario on his own. Facial expressions serve to embody the progress of the thought process and a change of stance. The visible formation of the recipient's stance enables the other participants to observe "online" how an argument is received by one party. This enables them to anticipate at an early stage how the perspectives of those involved relate to each other. In the present case, this resulted in a concerted display of consent (line 134/135). Recipient displays of "doing thinking" thus have the potential to act as a catalyst for the decision-making processes of groups.

\section{Summary}

The multimodal gestalts of the recipients' embodied displays of thinking resembled those of the speakers. Table 2 summarizes the findings.

On the whole, the embodiment of critical thinking is more prominent than that of co-imagining a scenario described by the current speaker: it contains not only a stylized thinking posture, but is also preceded by frowns. The greater prominence allows the recipient to point out problematic aspects while the speaker is still talking. In contrast, the lower salience of embodiments of 
TABLE 2 | Recipients' displays of "doing thinking."

\begin{tabular}{|c|c|c|c|c|}
\hline $\begin{array}{l}\text { Embodying independent } \\
\text { and critical thinking }\end{array}$ & thinking posture & $\begin{array}{l}\text { imaginative gaze and } \\
\text { wandering of the eyes }\end{array}$ & $\begin{array}{l}\text { prefaced by frowns, } \\
\text { emerging at the end of the } \\
\text { speaker's multi-unit turn and } \\
\text { lasting over the pause }\end{array}$ & $\begin{array}{l}\text { solitary } \\
\text { displacement/imagination; } \\
\text { projecting disagreement } \\
\text { and claim of the floor }\end{array}$ \\
\hline $\begin{array}{l}\text { Co-imagining a scenario } \\
\text { described by the current }\end{array}$ & inflexible posture & $\begin{array}{l}\text { imaginative gaze and } \\
\text { wandering of the eyes }\end{array}$ & $\begin{array}{l}\text { accompanying the } \\
\text { speaker's multi-unit turn }\end{array}$ & $\begin{array}{l}\text { joint imagination; } \\
\text { demonstrating agreement }\end{array}$ \\
\hline
\end{tabular}

speaker

co-imagining ensures that the attention of the current speaker is not distracted and that he can finish his multi-unit turn relatively undisturbed.

Both practices show that recipients are actively shaping the emerging interaction. The visible formation of the recipient's stance or knowledge enables both the current speaker and the other recipients to observe "online" how an argument is received by one party. In this way, recipient displays of "doing thinking" enable participants to anticipate different perspectives and to plan their next moves. They may thus act as a catalyst for the decision-making processes of groups.

\section{DISCUSSION}

Based on the seminal paper by Goodwin and Goodwin (1986) on thinking faces in word searches and recent conversational analytical studies on facial expressions (Peräkylä and Ruusuvuori, 2006; Ruusuvuori and Peräkylä, 2009; Kaukomaa et al., 2013, 2014, 2015) in face-to-face interactions, this paper investigated the epistemic and interactive functions of embodied displays of "doing thinking" in processes of argumentative decision-making. Using a corpus of video-recorded peer interactions, the study uncovered different practices of displaying thinking. The analysis showed that the embodied displays are context-sensitive and temporally coordinated with speech. Another finding is that thinking displays are not restricted to the face, but involve multiple resources. Among them are stylized thinking postures and imaginative gaze. Through marked contrasts between mobile and inflexible postures, the performers indicate the alignment of their attention to a world of thought, and vivid eye movements are used to evoke the impression of an ongoing thinking process. This shows that embodied displays of thinking are complex and highly dynamic multimodal gestalts.

The ways in which the embodied displays were used in interaction suggests that they should not be conceptualized as an external manifestation of internal processes. Regardless of whether they were produced by the speaker or the recipient, "doing thinking" was always organized as a public practice and multiparty activity: they were performed for the co-participants and served to mobilize their (visual) attention. In all examples presented here, it could be observed that the co-participants oriented toward the performer. In multiparty interactions, they are therefore an important resource for involving different parties in the activity-in-progress and shaping the emerging participation framework. In this respect, thinking displays fulfill essential interactive functions as assumed by Bavelas et al. (2014).

I shall argue, however, that embodied displays of "doing thinking" have other repercussions as well. By mobilizing the participants' attention, they create a space for the speaker to envision a hypothetical scenario and involve the participants in imagining potential consequences. Thinking displays thus seem to be ideally suited to constitute an epistemic ecology for exploring ideas and collaborative reasoning. This is of crucial importance for an exploratory framing of processes of argumentative decision-making.

Furthermore, investigating thinking displays in argumentative activities revealed that they also fulfill epistemic functions. When they were temporally aligned with lexical, syntactical, and/or morphological markers of epistemic modality, they were used to signal a thoughtful and tentative, independent yet cooperative, determined, affirmative or critical stance. Most importantly, due to their capacity to accompany longer stretches of talk and to change dynamically, they lend themselves to enact changes in the state of knowledge or stance. When recipients used thinking displays to mark their epistemic stance with regard to the speaker's position, this also implied showing that the other's ideas somehow affected their own thinking. This was particularly evident when recipients reciprocated the speaker's thinking displays (examples 1, 2, and 5). In this way, they emphasized the "jointness of emerging decisions" (Stevanovic et al., 2017).

The analysis is based on interaction data from children. However, there is little reason to assume that the practices reconstructed here are child-specific. For one thing, the analysis deliberately focused on older children who already have welldeveloped discursive skills. For another, the functions that the displays perform are also relevant in adult decisionmaking discourses. In this respect, the embodied displays are likely to prove a highly functional resource for adults as well.

This paper has presented multimodal and sequential analyses of embodied displays of "doing thinking" in a particular discursive practice. In order to fully understand thinking displays in naturally occurring interaction, future studies should examine their use in a range of different settings and discursive practices. Another area of future research concerns the acquisition of embodied argumentation. Assuming that the acquisition of discursive skills inherently involves the coordination of multimodal resources, the question arises as to how younger 
children come to use embodied thinking displays in different discursive activities. It can be expected that the conversational use of the "personal front" (Goffman, 1963) is a rather late achievement, because while multimodality is a resource, it is also a complex skill that itself needs to be acquired. This seems to apply especially to complex and dynamic thinking displays. Moreover, their interactive and epistemic functions are intricately interwoven with the discursive practice they are used for. Therefore, their acquisition should be closely related to the development of discursive skills. By describing the interactive and epistemic functions of thinking displays, the present article hopes to have created a first basis for investigating their acquisition.

\section{DATA AVAILABILITY STATEMENT}

The datasets presented in this article are not readily available because participants did not give written consent to share the data. Requests to access the datasets should be directed to Vivien Heller, vheller@uni-wuppertal.de.

\section{ETHICS STATEMENT}

Ethical review and approval was not required for the study on human participants in accordance with the local legislation and institutional requirements. Written informed consent to

\section{REFERENCES}

Antaki, C. (1994). Explaining and Arguing. The Social Organization of Accounts. London: Sage.

Arendt, B. (2019). Discourse acquisition in peer talk-the case of argumentation among kindergartners. Learn. Cult. Soc. Interact. 23:100341. doi: 10.1016/j.lcsi.2019.100342

Bavelas, J., and Chovil, N. (2000). Visible acts of meaning: an integrated message model of language in face-to-face dialogue. J. Lang. Soc. Psychol. 19, 163-194. doi: 10.1177/0261927X00019002001

Bavelas, J., and Chovil, N. (2018). Some pragmatic functions of conversational facial gestures. Gesture 17, 98-127. doi: 10.1075/gest.00012.bav

Bavelas, J., Gerwing, J., and Healing, S. (2014). "Including facial gestures in gesture-speech ensembles," in From Gesture in Conversation to Visible Action as Utterance, eds M. Seyfeddinipur and M. Gullberg (Amsterdam: Benjamins), 15-34.

Bergmann, J. R., and Luckmann, T. (1995). "Reconstructive genres of everyday communication," in Aspects of Oral Communication, ed U. M. Quasthoff (Berlin: de Gruyter), 289-304.

Birdwhistell, R. L. (1970). Kinesics and Context. Essays on Body Motion Communication. Philadelphia, PA: University of Pennsylvania Press.

Bova, A., and Arcidiacono, F. (2015). Beyond conflicts. Origin and types of issues leading to argumentative discussions during family mealtimes. J. Lang. Aggress. Confl. 3, 263-288. doi: 10.1075/jlac.3.2.02bov

Bühler, K. ([1934] 1999). Sprachtheorie. Die Darstellungsfunktion der Sprache. Stuttgart: Lucius and Lucius.

Chovil, N. (1991). Discourse-oriented facial displays in conversation. Res. Lang. Soc. Interact. 25, 163-194. doi: 10.1080/08351819109389361

Chovil, N. (1997). "Facing others: a social communicative perspective on facial displays," in The Psychology of Facial Expression, eds J. A. Russell and J. M. Fernández Dols (Cambridge: Cambridge University Press), 321-333. participate in this study was provided by the participants' legal guardian/next of kin.

\section{AUTHOR CONTRIBUTIONS}

VH developed the theoretical framework, collected the data with her team, and carried out the empirical analysis.

\section{FUNDING}

This research was funded by the ZEFFT of the University of Wuppertal as part of the project Epistemic Stance and Multimodal Discourse Acquisition.

\section{ACKNOWLEDGMENTS}

I would like to thank the participants of this study and the members of my project team, in particular Noelle Kinalzik, Nora Schönfelder and Denise Wakke, for data collection and helpful comments on the analyses. Further thanks go to Jan Schürmann for anonymizing the images.

\section{SUPPLEMENTARY MATERIAL}

The Supplementary Material for this article can be found online at: https://www.frontiersin.org/articles/10.3389/fpsyg. 2021.636671/full\#supplementary-material

Clark, H. H., and Fox Tree, J. E. (2002). Using uh and um in spontaneous speaking. Cognition 84, 83-111. doi: 10.1016/S0010-0277(02)00017-3

Crivelli, C., and Fridlund, A. J. (2018). Facial displays are tools for social influence. Trends Cogn. Sci. 22, 388-399. doi: 10.1016/j.tics.2018.02.006

Danby, S., and Theobald, M. (eds.). (2014). Disputes in Everyday Life. Social and Moral Orders of Children and Young People. Bingley: Emerald Publishing.

Darwin, C. (1872). The Expression of Emotions in Man and Animals. London: Murray.

Ehlich, K. (2014). "Argumentieren als sprachliche ressource des diskursiven Lernens," in Diskursive und Textuelle Strukturen in der Hochschuldidaktik, eds A. Hornung, G. Carobbio, and D. Sorrentino (Münster: Waxmann), 41-54.

Ekman, P. (1979). "About brows: emotional and conversational signals," in Human Ethology, eds M. von Cranach, K. Foppa, W. Lepenies, and D. Ploog (Cambridge: Cambridge University Press), 169-249.

Ekman, P., and Friesen, W. (1969). The repertoire of nonverbal behavior: categories, origins, usage, and coding. Semiotica 1, 49-98. doi: 10.1515/semi.1969.1.1.49

Ekman, P., and Friesen, W. (1975). Unmasking the Face. Englewood, CO: Prentice Hall.

Goffman, E. (1963). Behavior in Public Places. New York, NY: Free Press.

Goodwin, C. (1981). Conversational Organization: Interaction Between Speakers and Hearers. New York, NY: Academic Press.

Goodwin, C. (1987). Forgetfulness as an interactive resource. Soc. Psychol. Q. 50, 115-135. doi: $10.2307 / 2786746$

Goodwin, C. (2000). Action and embodiment within situated human interaction. J. Pragmat. 32, 1489-1522. doi: 10.1016/S0378-2166(99)00096-X

Goodwin, C. (2003). "The body in action," in Discourse, the Body, and Identity, eds J. Coupland and R. Gwyn (Basingstoke: Houndmills), 19-42.

Goodwin, M. H. (1980). Processes of mutual monitoring implicated in the production of description sequences. Soc. Inq. 50, 303-317. doi: 10.1111/j.1475-682X.1980.tb00024.x 
Goodwin, M. H. (2017). "Haptic sociality. The embodied interactive constitution of intimacy through touch," in Intercorporeality. Emerging Socialities in Interaction, eds C. Meyer, J. Streeck, and J. Scott Jordan (Oxford: Oxford University Press), 73-102.

Goodwin, M. H., Cekaite, A., and Goodwin, C. (2012). "Emotion as stance," in Emotion in Interaction, eds A. Peräkylä and M. L. Sorjonen (Oxford: Oxford University Press), 16-41.

Goodwin, M. H., and Goodwin, C. (1986). Gesture and coparticipation in the activity of searching for a word. Semiotica 62, 51-75. doi: 10.1515/semi.1986.62.1-2.51

Goodwin, M. H., and Goodwin, C. (1987). "Children's arguing." in Language, Gender, and Sex in Comparative Perspective, eds S. Philips, S. Steele, and C. Tanz (Cambridge: Cambridge University Press), 200-248.

Hanks, W. F. (1996). Language and Communicative Practices. Boulder, CO: Westview Press.

Hannken-Illjes, K., and Bose, I. (2019). Children in argumentation between antagonistic and cooperation. Informal Log. 39, 465-495. doi: $10.22329 /$ il.v39i4.6028

Heath, C., and Luff, P. (2013). "Embodied action and organizational activity," in The Handbook of Conversation Analysis, eds J. Sidnell and T. Stivers (Chichester: Wiley-Blackwell), 283-307.

Heath, C., vom Lehn, D., Cleverly, J., and Luff, P. (2012). "Revealing surprise: the local ecology and the transposition of action," in Emotion in Interaction, eds A. and M. L. Sorjonen (Oxford: Oxford University Press), 212-234.

Heller, V. (2014). Discursive practices in family dinner talk and classroom discourse. A contextual comparison. Learn. Cult. Soc. Interact. 3, 134-145. doi: 10.1016/j.lcsi.2014.02.001

Heller, V. (2018). Embodying epistemic responsibility. The interplay of gaze and stance-taking in children's collaborative reasoning. Res. Child. Soc. Interact. 2, 262-285. doi: 10.1558/rcsi.37391

Heller, V. (2019). Embodied displacements in young German children's storytelling. Layering of spaces, voices, and bodies. Res. Child. Soc. Interact. 3 , 168-195. doi: 10.1558/rcsi.37311

Heritage, J. (1984). "A change-of-state token and aspects of its sequential placement," in Structures of Social Action. Studies in Conversation Analysis, eds J. M. Atkinson and J. Heritage (Cambridge: Cambridge University Press), 299-345

Heritage, J. (2012). The epistemic engine: sequence organization and territories of knowledge. Res. Lang. Soc. Interact. 45, 30-52. doi: 10.1080/08351813.2012.646685

Jacquin, J. (2017). "Embodied argumentation in public debates. The role of gestures in the segmentation of argumentative moves," in Multimodal Argumentation and Rhetoric in Media Genres, eds A. Tseronis and C. Forceville (Amsterdam/Philadelphia, PA: John Benjamins Publishing), 239-262.

Kärkkäinen, E. (2006). Stance taking in conversation. From subjectivity to intersubjectivity. Text Talk 26, 699-731. doi: 10.1515/TEXT.2006.029

Kaukomaa, T., Peräkylä, A., and Ruusuvuori, J. (2013). Turn-opening smiles: facial expression constructing emotional transition in conversation. J. Pragmat. 55, 21-42. doi: 10.1016/j.pragma.2013.05.006

Kaukomaa, T., Peräkylä, A., and Ruusuvuori, J. (2014). Foreshadowing a problem. Turn-opening frowns in conversation. J. Pragmat. 71, 132-147. doi: 10.1016/j.pragma.2014.08.002

Kaukomaa, T., Peräkylä, A., and Ruusuvuori, J. (2015). How listeners use facial expression to shift the emotional stance of the speaker's utterance. Res. Lang. Soc. Interact. 48, 319-341. doi: 10.1080/08351813.2015.1058607

Keisanen, T. (2007). "Stancetaking as an interactional activity: challenging the prior speaker, in Stancetaking in discourse," in Subjectivity, Evaluation, Interaction, ed R. Englebretson (Philadelphia, PA: John Benjamins), 253-282.

Kendon, A. (1967). Some functions of gaze-direction in social interaction. Acta Psychol. 26, 22-63. doi: 10.1016/0001-6918(67)90005-4

Kendon, A. (1973). "The role of visible behavior in the organization of social interaction," in Social Communication and Movement: Studies of Interaction and Expression in Man and Chimpanzee, eds C. M. Cranach and I. Vine (New York, NY: Academic Press), 29-74.

Kendon, A. (1976). Some functions of the face in a kissing round. Semiotica 15, 299-334. doi: 10.1515/semi.1975.15.4.299

Kendon, A. (1990). Conducting Interaction. Patterns of Behavior in Focused Encounters. Cambridge: Cambridge University Press.
Kendon, A. (2004). Gesture. Visible Action as Utterance. Cambridge: Cambridge University Press.

Kidwell, M. (2013). "Framing, grounding, and coordinating conversational interaction: Posture, gaze, facial expression, and movement in space," in BodyLanguage-Communication. An International Handbook on Multimodality in Human Interaction, eds C. Müller, A. J. Cienki, E. Fricke, S. H. Ladewig, D. McNeill, and S. Tessendorf (Boston, MA: de Gruyter), 100-112.

Kinalzik, N., and Heller, V. (2020). Establishing joint imagined spaces in game explanations. Differences in the use of embodied resources among primary school children. Res. Child. Soc. Interact. 4, 28-50.

Kleist, H. von (1805). "Über die allmähliche Verfertigung der Gedanken beim Reden," in Sämtliche Werke und Briefe, II, ed H. Sembdner (München: Deutscher Taschenbuch Verlag), 319-324.

Knoblauch, H. (1991). "The taming of foes: the avoidance of asymmetry in informal discussions," in Asymmetries in Dialogue, eds I. Markovà and K. Foppa (Birmingham: Harvester Wheatsheaf; New York, NY: Barnes and Noble), 166-194.

Kreuz, J., and Luginbühl, M. (2020). From flat propositions to deep co-constructed and modalized argumentations: oral argumentative skills among elementaryschool children from grades 2 to 6. Res. Child. Soc. Interact. 4, 93-114. doi: $10.1558 /$ rcsi. 12416

Mirivel, J. C. (2011). "Embodied arguments: verbal claims and bodily evidence," in Embodied Interaction. Language and Body in the Material World, eds J. Streeck, C. Goodwin, and C.D. LeBaron (New York, NY: Cambridge University Press), 254-263.

Mondada, L. (2011). Understanding as an embodied, situated, and sequential achievement in interaction. J. Pragmat. 43, 542-552. doi: 10.1016/j.pragma.2010.08.019

Mondada, L. (2014). The local constitution of multimodal resources for social interaction. J. Pragmat. 65, 137-156. doi: 10.1016/j.pragma.2014.04.004

Mondada, L. (2018). Multiple temporalities of language and body in interaction. Challenges for transcribing multimodality. Res. Lang. Soc. Interact. 51, 85-106. doi: $10.1080 / 08351813.2018 .1413878$

Morek, M. (2020). Learning to modalize is learning to reason: on the role of epistemic modalizations in parent-child-talk and in written argumentation of secondary school students. Res. Child. Soc. Interact. 4, 115-141. doi: $10.1558 /$ rcsi. 12418

Müller, C. (2004). "Forms and uses of the Palm Up Open Hand: a case of a gesture family?" in The Semantics and Pragmatics of Everyday Gestures, eds C. Müller and R. Posner (Berlin: Weidler), 233-256.

Mundwiler, V., and Kreuz, J. (2018). "Collaborative decision-making in argumentative group discussions among primary school children, in Argumentation and Language," in Linguistic, Cognitive, and Discursive Explorations, eds S. Oswald, T. Herman, and J. Jacquin (Cham: Springer), 263-285.

Peräkylä, A., and Ruusuvuori, J. (2006). "Facial expression in an assessment," in Video Analysis: Methodology and Methods. Qualitative Audiovisual Data Analysis in Sociology, ed H. Knoblauch (Frankfurt am Main: Lang), 127-142.

Peräkylä, A., and Ruusuvuori, J. (2012). "Facial expression and interactional regulation of emotion," in Emotion in Interaction, eds A. Peräkylä and M. L. Sorjonen (New York, NY: Oxford University Press), 64-91.

Pomerantz, A. (1984). "Agreeing and disagreeing with assessments: some features of preferred/dispreferred turn shapes," in Structures of Social Action. Studies in Conversation Analysis, eds J. M. Atkinson and J. Heritage (Cambridge: Cambridge University Press), 57-101.

Quasthoff, U., Heller, V., and Morek, M. (2017). On the sequential organization and genre-orientation of discourse units in interaction. An analytic framework. Discourse Stud. 19, 84-110. doi: 10.1177/1461445616683596

Reineke, S. (2016). Wissenszuschreibungen in der Interaktion. Eine gesprächsanalytische Untersuchung impliziter und expliziter Formen der Zuschreibung von Wissen. Heidelberg: Universitätsverlag Winter.

Ricci Bitti, P. E. (2014). "Facial expression and social interaction," in BodyLanguage-Communication. An International Handbook on Multimodality in Human Interaction, eds C. Müller, A. J. Cienki, E. Fricke, S. H. Ladewig, D. McNeill, and S. Tessendorf (Berlin, Boston, MA: de Gruyter Mouton), 1342-1349.

Rossano, F. (2012). Gaze Behavior in Face-to-Face Interaction. Nijmegen: Radboud Repository 
Rossano, F. (2013). "Gaze in conversation," in The Handbook of Conversation Analysis, eds J. Sidnell and T. Stivers (Chichester: Wiley-Blackwell), 308-329.

Ruusuvuori, J., and Peräkylä, A. (2009). Facial and verbal expressions in assessing stories and topics. Res. Lang. Soc. Interact. 42, 377-394. doi: $10.1080 / 08351810903296499$

Sacks, H., and Jefferson, G. (1992). Lectures on Conversation. Oxford: Blackwell.

Sacks, H., Schegloff, E. A., and Jefferson, G. (1974). A simplest systematics for the organization of turn-taking for conversation. Language 50, 696-735. doi: 10.1353/lan.1974.0010

Scheflen, A. E. (1965). The significance of posture in communication systems. Psychiatry 27, 316-331. doi: 10.1080/00332747.1964.11023403

Schönfelder, N., and Heller, V. (2019). "Embodied reciprocity in conversational argumentation. Soliciting and giving reasons with Palm Up Open Hand gestures," in Proceedings of the 6th Gesture and Speech in Interaction GESPIN, eds A. Grimminger (Paderborn: Universitätsbibliothek), 81-86.

Selting, M., Auer, P., Dagmar Barth-Weingarten, D., Bergmann, J. R., Bergmann, P., Birkner, K., et al. (2011). A system for transcribing talk-in-interaction: GAT 2. Translated and adapted for English by Elizabeth Couper-Kuhlen and Dagmar Barth-Weingarten. Gesprächsforschung Online Zeitschrift verbalen Interaktion $12,1-51$.

Sidnell, J. (2012). "Who knows best?": Evidentiality and epistemic asymmetry in conversation. Pragmat. Soc. 3, 294-320. doi: 10.1075/ps.3.2.08sid

Sterponi, L. (2009). Accountability in family discourse. Childhood 16, 441-459. doi: $10.1177 / 0907568209343269$

Stevanovic, M. (2012). Establishing joint decisions in a dyad. Discourse Stud. 14, 779-803. doi: 10.1177/1461445612456654

Stevanovic, M., Himberg, T., Niinisalo, M., Kahri, M., Peräkylä, A., Sams, M., et al. (2017). Sequentiality, mutual visibility, and behavioral matching: body sway and pitch register during joint decision making. Res. Lang. Soc. Interac. 50, 33-53. doi: 10.1080/08351813.2017.1262130

Stivers, T., Mondada, L., and Steensig, J. (2011). "Knowledge, morality and affiliation in social interaction," in The Morality of Knowledge in Conversation, eds T. Stivers, L. Mondada, and J. Steensig (Cambridge: Cambridge University Press), 3-24.

Streeck, J. (2007). Geste und verstreichende Zeit: innehalten und Bedeutungswandel der "bietenden Hand", ' in Gespräch als Prozess. Linguistische Aspekte der Zeitlichkeit verbaler Interaktion, ed H. Hausendorf (Tübingen: Narr), 157-177.

Streeck, J. (2009). Forward-gesturing. Discourse Process. 46, 161-179. doi: $10.1080 / 01638530902728793$

Streeck, J. (2011). Gesturecraft. The Manufacture of Meaning. Philadelphia, PA: John Benjamins.

Streeck, J., Goodwin, C., and LeBaron, C. D. (2011). "Embodied interaction in the material world: an introduction," in Embodied Interaction. Language and Body in the Material World, eds J. Streeck, C. Goodwin, and C. D. LeBaron (New York, NY: Cambridge University Press), 1-26.

Stukenbrock, A. (2017). "Intercorporeal phantasms: kinesthetic alignment with imagined bodies inself-defense training," in Intercorporeality. Emerging Socialities in Interaction, eds C. Meyer, J. Streeck, and J. S. Jordan (Oxford: Oxford University Press), 237-263.

Weiß, C., and Auer, P. (2016). Das Blickverhalten des Rezipienten bei Sprecherhäsitationen: eine explorative Studie. Gesprächsforschung Online Zeitschrift verbalen Interaktion 17, 32-167.

Conflict of Interest: The author declares that the research was conducted in the absence of any commercial or financial relationships that could be constructed as a potential conflict of interest.

Copyright (๑) 2021 Heller. This is an open-access article distributed under the terms of the Creative Commons Attribution License (CC BY). The use, distribution or reproduction in other forums is permitted, provided the original author(s) and the copyright owner(s) are credited and that the original publication in this journal is cited, in accordance with accepted academic practice. No use, distribution or reproduction is permitted which does not comply with these terms. 


\section{The Psychophysiological Experience of Solving Moral Dilemmas Together: An Interdisciplinary Comparison Between Participants With and Without Depression}

\author{
Emmi Koskinen ${ }^{1 *}$, Samuel Tuhkanen ${ }^{3}$, Milla Järvensivu ${ }^{1}$, Enikö Savander ${ }^{2}$, Taina Valkeapää ${ }^{1}$, \\ Kaisa Valkia ${ }^{1}$, Elina Weiste ${ }^{3}$ and Melisa Stevanovic ${ }^{4}$ \\ ${ }^{1}$ University of Helsinki, Faculty of Social Sciences, Helsinki, Finland, ${ }^{2}$ Päijät-Häme Central Hospital, Department of Psychiatry, \\ Lahti, Finland, ${ }^{3}$ University of Helsinki, Faculty of Arts, Helsinki, Finland, ${ }^{4}$ Tampere University, Faculty of Social Sciences, Tampere, \\ Finland
}

OPEN ACCESS

Edited by:

Antonio Bova,

Catholic University of the Sacred

Heart, Italy

Reviewed by:

Ksenija Krstic,

Faculty of Philosophy, University of

Belgrade, Serbia

Marzia Saglietti,

University of Bologna, Italy

${ }^{*}$ Correspondence:

Emmi Koskinen

emmi.ek.koskinen@helsinki.fi

Specialty section:

This article was submitted to

Language Sciences,

a section of the journal

Frontiers in Communication

Received: 04 November 2020

Accepted: 14 January 2021

Published: 26 February 2021

Citation:

Koskinen E, Tuhkanen S,

Järvensivu M, Savander $E$,

Valkeapää $T$, Valkia $K$, Weiste $E$ and

Stevanovic M (2021) The

Psychophysiological Experience of

Solving Moral Dilemmas Together: An

Interdisciplinary Comparison Between

Participants With and

Without Depression.

Front. Commun. 6:625968.

doi: 10.3389/fcomm.2021.625968
Dyads with a depressed and a non-depressed participant $(N=15)$ and two nondepressed participants $(N=15)$ discussed a moral dilemma, during which the participants' gaze direction and skin conductance (SC) were measured. Partner gazing occurred most frequently when a speaker took a strong stance toward saving a person in the dilemma, depressed participants however looking at their co-participants less often than non-depressed participants. The participants' SC response rates were higher during responsive utterances expressing disagreement (vs. agreement) with co-participant ideas or suggesting that a person be sacrificed (vs. saved). We argue that a better understanding of the affective corollaries of human social interaction necessitates a balanced consideration of both contents of talk and behavioral patterns.

Keywords: social interaction, experimentation, interdisciplinary research, gaze, depression, psychophysiology, moral dilemma

\section{INTRODUCTION}

Choosing the least of several evils is a common everyday challenge, which is likely to provoke anxiety and arousal in most individuals. It is therefore only to be expected that people tend to discuss their dilemmatic situations and the different choices that they entail with other people, instead of mulling over them in solitude. This may be assumed to be the case particularly in those situations where the decision-making is intertwined with complex moral considerations. It is therefore remarkable that, although there are large bodies of studies on solving moral dilemmas as an individual phenomenon (see Christensen et al., 2014), there are only few studies addressing moral problem solving in social interactional encounters (see, however, Lavelle et al., 2014). Furthermore, the experience of solving moral dilemmas may be distinct in depression, due to the increased threat arousal and pathological worry that have been associated with the condition (Starcevic, 1995), but not much is known about how this might show when solving these types of problems together with others. In addition, the field of empirical social interaction research has been divided in that the researchers typically focus either on the content of talk (e.g., Bales, 1950; Levenson and Gottman, 1983; Luminet et al., 2000; Zech and Rimé, 2005; Smirnov et al., 2019) or on the patterns of the turn-by-turn unfolding of interaction that are independent of the specific contents of utterances (e.g., Schegloff, 2007; Sidnell, 2014; Arundale, 
2020). Here, we argue that a better understanding of human social interaction necessitates a balanced consideration of both of these aspects of it. Drawing on data from an experiment where participants with and without depression discussed a moral dilemma, which required them to make a decision to sacrifice a person in order to save others, we examine, how both the contents of talk and the patterns of gaze and the turn-by-turn unfolding of conversational utterances are underpinned by the participants' physiological arousal responses during the conversation.

\section{Solving Moral Dilemmas Together}

Moral dilemmas have become a standard methodology for research on moral judgment (Christensen et al., 2014). Moral dilemmas are hypothetical short stories, which describe a situation in which conflicting moral reasons are relevant (e.g., Foot, 1967; also see; Thomson, 1976). Traditional theories of moral development (see Kohlberg, 1969) have emphasized the role of controlled cognition in the maturation of moral judgment. In general, solutions to moral dilemmas have been clarified with reference to two different philosophical ethics: utilitarianism or deontology. Utilitarian judgments (Mill, 1998) focus on "the greater good" in the outcome and aim at maximizing benefits for the largest number of people. The deontological perspective (Kant, 1959), in contrast, highlights one's obligations and responsibilities towards other people (e.g., the imperative not to kill), which can trump utilitarian considerations (Greene et al., 2008).

The conflicting types of moral judgment have been compared and examined in their own right, studies showing that utilitarian judgments are rational and unemotional (Lee and Gino, 2015) and require a high working memory capacity (Moore et al., 2008). Deontological judgments, then again, have been suggested to indicate intuitive and emotional processes (Greene et al., 2008; for a review, see ; Christensen and Gomila, 2012), motivated by one's relationships and personal pursuits towards other people (cf. Scheffler, 2010). The decision-makers' judgments have been shown to be influenced by different parameters of the moral dilemma task, such as psychological and emotional distance, concreteness and visuality, as well as the existence of time pressure (Amit and Greene, 2012; Aguilar et al., 2013; Körner and Volk, 2014). Some researchers have also investigated and compared the task-related emotional arousal of the participants dealing with different types of dilemmas by using self-report measures (Christensen et al., 2014).

In all the diverse above-mentioned studies, the moral dilemmas are directed to one "decision-maker" (Kvalnes, 2019). However, if the moral dilemma task is solved in interaction with another participant, the situation becomes inherently much more complex. In order to address moral decision-making as a social interactional phenomenon with its specific behavioral and emotional processes, some researchers have used the so-called "balloon task" to stimulate conversation between participants in experimental settings (e.g., Purver et al., 2003; McCabe and Lavelle, 2012; Lavelle et al., 2013; Lavelle et al., 2014; Howes et al., 2016). In the balloon task, which we also use in the current study, participants are presented with a fictional scenario in which a hot-air balloon is losing altitude and is about to crash. The only way for any of the three passengers of the balloon to survive is that one of them jumps to a certain death. The three passengers are: a cancer scientist, a pregnant primary school teacher, and the husband of the teacher, who is also the pilot of the balloon. The balloon task has been deemed effective in generating debates between the participants in interaction (Purver et al., 2003, p. 6). In the previous studies using the balloon task, the investigation has however focused merely on the patterns of interactional behavior unrelated to the content of the moral dilemma task, such as the use of clarification questions (Purver et al., 2003) and the practices of participation and nonverbal communication (Lavelle et al., 2013; Lavelle et al., 2014). In this paper, in contrast, our focus will be on the core aspect of solving moral dilemmas in social interaction: making proposals with different contents and varying degrees of expressive strength, defending these proposals through different types of arguments, agreeing or disagreeing with the arguments of the co-participant, and, finally, negotiating a joint decision.

Each of the above-listed conversational actions, which constitute the activity of solving moral dilemmas together, can have significant affective corollaries. A proposal as an "initiating utterance" is a powerful conversational action, which entails, not only a claim of the right to have a word to say in the matter at hand, but also a claim of the right to determine the content of the participants' local interactional agenda. Proposal speakers are sensitive to these implicit claims, orienting to a need to mitigate their proposals and their implicit claims of power in various ways (Stevanovic 2013; Stevanovic 2015). Furthermore, in the context of the balloon task, the mere content of the proposal - that is, the question of who should jump to death - can in itself be an arousing matter to say aloud in the presence of another person. Initiating utterances, in turn, make relevant "responsive utterances", which, in the case of proposals, may either agree or disagree with the arguments presented in the proposal. While there are "sociable arguments" (Schiffrin 1984; Schiffrin 1990) and specific conversational contexts, such as radio or television talk shows (Hutchby, 1996; Thornborrow, 2015), where controversies are highly expected, it may be generally assumed that speakers tend to avoid argument and disagreement. This is shown in the participants orienting to a need to mitigate the facethreatening implications of their differences of opinion (Goffman, 1955; Brown and Levinson, 1987) and to display an overall preference for agreement, for example, by producing their dispreferred responses with delay (Pomerantz, 1984). All this suggests that the production of disagreeing turns in response to proposals is something that the participants themselves perceive as interactionally problematic. Finally, also the reaching of a joint decision can be an arousing interactional event - especially, when the participants feel responsible for its content (Stevanovic et al., under review) and, presumably, also when the content of the decision in itself has affective salience, as is the case in the context of solving moral dilemmas.

One important resource that participants use to regulate the affective corollaries of their utterances is gaze. First, prior literature on the use of gaze in face-to-face suggests that gaze 
can be used to regulate the interactional force of one's utterances. With reference to the notion of "mobilizing response" Stivers and Rossano (2010) suggested that, across various types of utterances, the speaker's gaze on the recipient increases the recipient's pressure to respond to the utterance. The response-mobilizing function of gaze is in line with the psychological literature where gaze directed straight to the co-participant is perceived as an indication of dominance (Argyle and Dean, 1965; Hall et al., 2005). When such dominance co-occurs with an utterance that potentially implies a decision, it may be perceived as strengthening the display of the speaker's commitment to it. It is a different thing to say "Let's do X" when you look at your coparticipants, compared to when you don't. Second, gaze withdrawal may be used to indicate and manage the relative delicacy of the content of the talk. While direct eye contact is common when the topic under the discussion is "easy" - that is, cognitively more straightforward and less personal (Argyle and Dean, 1965), people tend to direct their gaze away from the coparticipant when discussing a difficult topic or feeling uncertain or ashamed (Burgoon et al., 1996; Bente et al., 1998). Avoidance of mutual gaze is also more frequent when the social situation is experienced as threatening or anxiety provoking (Ewbank et al., 2009; Schulze et al., 2013). All this suggests that the investigation of the behavioral and emotional processes associated with moral decision-making should include the examination of the participants' use of gaze as a key aspect of their interactional behavior.

\section{Affective and Psychophysiological Underpinnings of Social Interaction}

Many studies of interaction have considered the specific contents or topics of participants' talk as the main target of investigation. In his pioneering work, Bales (1950) developed systematic methods of group observation and measurement of interaction processes, launching a coding system that classified group behavior into task-oriented and relationship-oriented interactions. Analogous coding schemes have also been used in a range of psychophysiological and neurological studies of social interaction. In a seminal study, Levenson and Gottman (1983) investigated discussions between marital couples. The authors employed a combination of measurements, such as heart rate, skin conductance and movement, to construct a combined measure to assess psychophysiological synchronicity between the participants, showing that this measure was higher when the participants were discussing their marital problems and lower when they were discussing more neutral topics. In a similar vein, Smirnov et al., (2019) investigated the synchronization of brain activity across speakers and listeners during the telling of emotional or neutral autobiographical stories. Contents and topics of social interaction have also been investigated from the point of view of people's subjective needs to talk about specific, affectively salient issues (e.g., Luminet et al., 2000; Zech and Rimé, 2005).

In contrast, empirical social interaction studies utilizing conversation analysis have mainly focused on describing the patterns of the turn-by-turn sequential unfolding of naturally- occurring interaction - that is, the chaining of conversational actions (such as requests, proposals, invitations) and their responses (such as acceptances and rejections) - such "structures of social action" (Atkinson and Heritage, 1984) having been considered as independent of the specific contents of talk (Schegloff, 2007; Sidnell, 2014; Arundale, 2020). A central advantage of the approach lies in its capacity to reveal participants' own orientations (emic) to what is going on in the encounter (see e.g., Garfinkel and Harvey, 1970: 345; Schegloff, 1997), instead of being based on the researcher's $a$ priori assumptions about the social world and interaction (etic).

During the most recent years, however, novel conversationanalytically informed research interests have emerged, which have also given rise to new types of theoretical and methodological challenges. On the one hand, contemporary measurement technologies such as motion capture (Edlund et al., 2013; Stevanovic et al., 2017) and eye-tracking (Dindar et al., 2017; Kendrick and Holler, 2017) have been seen as valuable tools to get detailed knowledge of participant behaviors. Using these technologies in a laboratory, however, involves a shift from naturally-occurring interactions toward more researchercontrolled realizations of the interactional encounters under investigation. Of course, knowledge about the basic structures of interaction may also be gained in these settings, but the task instructions and their potential influence on the results must be carefully considered (for a discussion on the "natural-contrived" continuum of producing social interaction data, see Speer, 2002).

In a similar vein, the rise of conversation-analytically informed interdisciplinary research endeavors concerning, for example, the kinds of prereflective, unconscious, or involuntary phenomena such as body sway (Stevanovic et al., 2017) or psychophysiological reactions (Peräkylä et al., 2015; Stevanovic et al., 2019; Stevanovic et al., 2021; Voutilainen et al., 2014) has made it necessary to move beyond the mere case-by-case qualitative analysis of interactional sequences to approaches involving coding and quantification, which enable the making of generalizations across multiple instances of data. This is necessary to be able to deal with the relatively high level of "noise" that is an inevitable part of these types of data. Coding and quantification, however, involves a risk of an epistemological shift from the emic towards the etic (e.g., Markee, 2012). Conversationanalytically informed researchers nonetheless seek to incorporate participants' own orientations in the coding schemes as far as is possible (for a discussion on the topic, see Stivers, 2015), and their studies have contributed to an increasing understanding of several, inherently emic concerns associated with the turn-byturn sequences of interaction. For example, Peräkylä and colleagues (2015) found that affiliative story reception is associated with a decrease in the storyteller's arousal and an increase in the story recipient's arousal, as measured by the participants' skin conductance (SC) responses. In a similar vein, Stevanovic and colleagues (under review) used a series of food-decision-making tasks, observing that the relinquishing of one's initially established preferences was associated with higher SC response rates than either acceptances or rejections of the coparticipants' proposals. Indeed, building on, extending, and contributing to the initial goal of conversation analysis, which 
is to reveal participants' own orientations to interactional events and behaviors, these studies have shed light on the psychophysiologically underpinned experiential aspects of precisely these types of orientations.

In this study, we consider the psychophysiological underpinnings of moral-decision-making interaction. In our view, the consideration of this type of interaction necessitates consideration of the specific contents of the participants' utterances. Solving a moral dilemma together involves each participant drawing on their own moral judgment, defending and opposing views that come across as justified or objectionable. Proposals in this context are thus not "just" proposals to be treated independent of what has been suggested, but the moral implications of these proposals may be tightly bound to their specific contents (e.g., saving or sacrificing an individual from the crashing balloon). But this context also makes it relevant to consider the more generic patterns of interactional conduct. When co-occurring with affectively salient contents, such as the ones that characterize moral-decision-making interaction, instances of agreement and disagreement may reverberate in the participants' bodies even more than they would do in more neutral everyday settings. In this sense, our study draws on both of the two broad traditions of empirical social interaction research described above, seeking to build a bridge between them.

\section{Depression and Social Interaction}

The Diagnostic and Statistical Manual of Mental Disorders (DSM-5, American Psychological Association, 2013) associates depression with loss of pleasure, feelings of worthlessness, indecisiveness, and thoughts of death. It is therefore not a surprise that text analysis methods have shown that those with symptoms of depression use excessive number of words conveying negative emotions (Tausczik and Pennebaker, 2010). A recent computerized big data text analysis conducted by Al-Mosaiwi and Johnstone (2018) examined the use of absolutist words, such as "always", "nothing" or "completely", and found that absolutist words were even better markers for mental health forums than negative emotion words. This was interpreted in relation to so-called "absolutist thinking", which has been suggested to underlie anxiety and depression (Beck, 1979; Burns, 1989; Williams and Garland, 2002). In addition, dichotomous thinking, cognitive rigidity, and problem-solving deficits have been repeatedly found to co-occur in suicidal individuals (see Ellis and Rutherford, 2008 for a review). In a more interactional perspective, studies on storytelling in therapeutic interactions and clinical interviews have identified specific depression-related language use, which highlight the feelings of helplessness, hopelessness, and low personal agency in the narratives of individuals with depression (Vanheule and Hauser, 2008; Angus and Greenberg, 2011; Ekberg and LeCouteur, 2015; Muntigl, 2016). It is an open question, however, whether the above-mentioned findings regarding expressions of negative affect and low personal agency characterize the conversational interactions of individuals with depression in non-clinical contexts, especially when individuals with depressive symptoms can be very skillful in hiding their condition (Kirk et al., 2000).
There is much research on how gaze behavior is altered in depression. Results of eye-tracking studies reveal that, compared to non-depressed controls, individuals with depression spend more time viewing negative images (e.g., sad faces) and less time with positive or neutral images (Kellough et al., 2008; Sanchez et al., 2013; Isaac, et al., 2014). Furthermore, research on clinical interviews has shown that patients with depression display less eye contact with mental health professionals than the patients with other psychiatric conditions such as schizophrenia (Jones and Pansa, 1979) and non-depressed controls (Hinchliffe et al., 1970; Sobin and Sackeim, 1997; Fiquer, et al., 2018). Interestingly, the avoidance of eye contact has been observed to emerge regardless of the severity of depression and to persist relatively long after treatment (Fiquer et al., 2018). In the context of social interaction, the gaze behavior of individuals with depression has been interpreted as withdrawal from social contacts and as avoidance of intimacy (Hinchliffe et al., 1970). Less is known, however, of whether and how the complexities of gaze behavior during the micro-phenomena of the turn-by-turn sequential unfolding of interaction might be modulated by depression. Conversation analytic research on social interaction deficits, such as autism spectrum disorder, has suggested that the deviances attributable to the clinical condition may sometimes instantiate themselves particularly at very specific moments of the interactional sequences (Wiklund, 2012). What is not yet known, however, is whether something like this could also characterize gaze behavior in depression.

Given the overall anchoredness of social interaction in embodied, emotional and psychophysiological processes (see Peräkylä et al., 2015), there may exist idiosyncratic patterns of psychophysiological responses for participants with depression engaged in social interaction. In general, depression is associated with dysregulation in both parasympathetic and sympathetic branches of the autonomic nervous system (ANS) (e.g., Rottenberg, 2007; Rottenberg et al., 2007; Kemp et al., 2010; Beauchaine 2015; Koenig et al., 2016; Sarchiapone et al., 2018; Brush et al., 2019). These idiosyncrasies include a flat or low SC profile (Vahey and Becerra, 2015), which seems to be a reliable feature of depression and a valid marker of suicidal risk (Sarchiapone et al., 2018), and is consistent with early theorizing considering the behavioral and physiological underarousal as a prominent part of depressive symptomatology (Grossberg, 1972; Benning and Ait Oumeziane, 2017). Many laboratory studies have also associated depression with alleviated reactions to negative and positive cues, such as winning or losing money in mock gambling paradigms (Henriques and Davidson, 1990; Henriques and Davidson, 2000; Sloan et al., 2001) or watching sad or amusing films (Rottenberg et al., 2002). While some researchers have thus considered alleviated reactivity to positive and negative social cues as a hallmark of major depressive disorder (see Henriques and Davidson, 1991; Rottenberg, 2005), this conclusion has been challenged in studies on the reactivity to social cues outside the laboratory. In these studies, individuals with depressions have been, in contrast, observed to display heightened sensitivity to both positive and negative social cues (Needles and Abramson, 
1990; Allen et al., 2004; Gilbert, 2006; Steger and Kashdan, 2009). It is therefore possible that, for example, the phenomena of increased threat arousal and pathological worry, which have been considered as a part of the etiology of depression (Starcevic, 1995), could show in heightened physiological arousal during conversational encounters. This contrasting hypothesis received support from our own earlier study (Stevanovic et al., under review), where we found that, during an affectively neutral conversational decision-making task, participants with depression exhibited generally higher SC response rates than their healthy comparisons. In this study, we consider whether this finding applies also to decision-making interactions with affectively more salient content.

\section{Research Question and Hypotheses}

In this study, we ask how the participants' interactional behavior during a dyadic moral dilemma task is reflected in their psychophysiological responses and gaze behavior. The more specific hypotheses, which we test empirically, are the following:

Hypothesis 1: We assume that transitions between activities necessitate heightened intersubjectivity (Stevanovic et al., 2017), which will be reflected in the participants' higher SC response rates during beginning and end phases of the moral dilemma task, compared to the middle phase.

Hypothesis 2: During the middle phase, the content of talk and the patterns of gaze and the turn-by-turn unfolding of conversational utterances are reflected in the participants' psychophysiological responses. Here, we make the following, more specific predictions: a). Given the previous association between physiological arousal and talk about affectively salient issues (Levenson and Gottman, 1983; Luminet et al., 2000; Zech and Rimé, 2005), we assume that utterances concerning a balloon passenger sacrificing him- or herself by jumping from the balloon, are associated with higher SC response rates in the speaker than utterances concerning the saving of a balloon passenger. b). Drawing on previous literature on the relationship between the delicacy of talk and gazial behavior (Argyle and Dean, 1965; Burgoon et al., 1996; Bente et al., 1998), we assume that strong "initiating utterances" - that is, utterances that present a specific proposal in favor of sacrificing or saving a person - are associated with more gazing towards the co-participant than the weak ones. c). Based on the idea of gaze having specific "response mobilizing" features (Stivers and Rossano, 2010), we assume that more gazing towards the co-participant is associated with faster recipient responses. d). Drawing on the notion of preference (Pomerantz, 1984) and on the assumption that the production of dispreferred actions may thus be experienced as affectively salient, we assume that "responsive utterances" that express disagreement with what the co-participant has said before are produced with delay and associated with higher SC response rates than responsive utterances that represent agreement.

Hypothesis 3: The contents of talk and the patterns of gaze and the turn-by-turn unfolding of conversational utterances, and the SC response rates may be different for participants with and without depression. Here, we make the following, more specific predictions: a). In accordance with earlier literature concerning depressed individuals' excessive use of negative emotion words and language conveying hopelessness and low personal agency (e.g., Tausczik and Pennebaker, 2010; Angus and Greenberg, 2011) we assume that participants with depression diagnosis make fewer strong (vs. weak) and initiating (vs. responsive) utterances in general but have a higher proportion of sacrificing (vs. saving) utterances than their non-depressed comparisons. b). Participants with depression exhibit less coparticipant gazing than their non-depressed comparisons, these differences being possibly most prevalent at specific, critical moments of interaction (e.g., Wiklund, 2012). c). SC response rates may be different for participants with and without depression. Given the mixed evidence so far, involving both the ideas of the physiological underarousal (Grossberg, 1972; Benning and Ait Oumeziane, 2017) and increased threat arousal and worry (Starcevic, 1995; Stevanovic et al., under review) as parts of depressive symptomatology, we refrain from making predictions about the direction of the effect.

\section{METHODS}

\section{Ethics}

Informed, written consent was given by all participants prior to study, after they had been informed about the aims of the study and about their rights to withdraw their consent anytime they wished (see below). Institutional Review Board approval was obtained from the Ethics Committee of the Helsinki University Central Hospital [June 18, 2018].

\section{Participants}

We recruited participants $(N=15)$ who had been diagnosed with middle stage depression within the past 12 months, and, as a comparison group, participants $(N=45)$ who had not got a depression diagnosis within the past ten years. The participants $(N=60)$ had at least five years (3 years if under 25) of working life experience and with at least one bachelor's degree or equivalent level of education. The participants were divided into two groups of pairs: 15 pairs, where one participant had a depression diagnosis ("case pair"), and 15 pairs, where neither participant had been diagnosed with depression within the past ten years (“comparison pair").

Participants were recruited through social media and the University of Helsinki mailing lists. Potential participants were asked for background information (age, education, work history, and earlier depression diagnosis) through a phone interview. Based on this information the candidate was either excluded from the research or guided to the group of participants with depression diagnosis or to the comparison group. The clinical status of the participants with depression diagnosis was confirmed by a medical specialist in psychiatry and general practice, who met each participant privately and did a clinical interview and needed inquiry on symptoms by using the Beck Depression Inventory (Beck et al., 1961) and Montgomery-Åsberg Depression Rating Scale (Montgomery and Åsberg, 1979). The medical specialist also took care of arranging treatment for the participants when needed. 
Before the experiment, both participants were guided to fill out a set of questionnaires and the purpose of the research was clarified verbally and in writing. The participants were informed about our focus being on the structures of decisionmaking interaction and on the impact of mood on its dynamics. The clinical status of those participants with depression diagnosis was not revealed to the interaction partner, because the information could have affected the dynamics of the subject of study and, furthermore, could have unnecessarily stigmatized these participants. At this point we also gave the participants the opportunity to ask questions about the research. The participants were informed about the researchers' obligation to maintain secrecy, the practices of anonymity and data management, the publication of the research results, and the voluntariness of participation in the research. The participants were also told that, even after the written consent, they could still withdraw their participation at any time without this affecting their position or treatment. The participants were also told how to reverse their consent in practice.

\section{Equipment}

Skin conductance (SC), as well as blood volume pulse (BVP), were measured from both participants at a $128 \mathrm{~Hz}$ sampling rate with NeXus-10 (Mind Media, Netherlands) devices. SC was measured via two foam electrodes that were placed on the medial side of the left foot. The BVP sensor was attached to the second digit of the left foot. Binocular head-mounted Pupil Labs eyetrackers (Pupil Labs UG haftungsbeschrnkt, Berlin, Germany) were used to record eye-movements from both participants at a $60 \mathrm{~Hz}$ sampling rate. The eye-trackers were simultaneously calibrated with 16 calibration markers that were presented one by on a LG OLED55C7V 55" monitor. The open-source Pupil Capture software (v1.8 from: https:// github.com/pupil-labs/pupil) was used to record and calibrate the eye tracker. In addition, Shimmer3 IMUs (Shimmer Sensing, Ireland, Dublin) were attached to the right wrist of each participant to record linear acceleration and angular velocity. The NeXus-10, Shimmer3 and Pupil recordings were synchronized via Unix timestamps with a custom-made software (https://github.com/samtuhka/InteractionExperimentController). Only skin conductance data and gaze data are analyzed in this paper.

\section{Experiment}

One pair of participants was studied at a time. As described in the Introduction, the participants were presented with a moral dilemma where they were asked to imagine a fictional scenario where a hot-air balloon with three passengers is losing altitude and about to crash. The only way for any of the passengers to survive is for one of them to jump to a certain death. The three passengers are: a scientist whose research could bring about a revolutionary treatment for cancer, a pregnant primary school teacher, and the husband of the teacher, who is also the balloon pilot. The participants were asked to come up with an agreed-upon decision on which one of the passengers should jump from the balloon.

The instructions and description of the task were presented verbally by one of the experimenters. No further instructions were provided on whether, for example, the two remaining passengers could steer the balloon without the pilot, or how much of his research the scientist may have shared with his colleagues. No time limits or other constraints were placed on the participants.

The participants also completed two other tasks that are not reported in this paper (see Stevanovic et al., 2021; Stevanovic et al., under review). The order of these tasks was counterbalanced across dyads. The eye trackers were calibrated between each trial. The participants sat facing each other at about an $120^{\circ}$ angle from each other. The angle was chosen so that the participants wouldn't have to change position to calibrate the eye-tracker.

At the beginning of each session, the participants were asked to fill in a set of questionnaires: (1) Locus of Control Scale (Rotter, 1966), (2) Self-Monitoring Scale (Snyder and Gandestad, 1986), (3) Empowerment Scale (Rogers et al., 2010), (4) Ten-Item Personality Inventory, TIPI (Gosling et al., 2003), as well as to answer questions about their perceptions and experiences of the task requirements, their interaction partner, and the dynamics of interaction.

\section{Annotations}

We used Praat (Boersma, 2001) to annotate the participants' interactional behavior during the moral dilemma task (see Supplementary Material for a more detailed description of the annotations). First, we broke each task down into three phases. The beginning phase starts when the experimenter stops giving the instructions to the participants and ends after $10 \mathrm{~s}$, during which the participants' usually give their first reactions to the task. The middle phase is where the participants negotiate about who should be sacrificed and who should be saved. In the end phase the participants' give their final reactions to the task after making the decision. The end phase starts at the moment when one of the participants begins to pronounce their final decision, summarizing what has been tentatively agreed upon previously (e.g., "Let's take the pilot") and ends when the participants stop discussing the task.

Second, during the middle phase of the task, we annotated the initiating utterances, where one of the participants presents a specific proposal concerning a person in the balloon. We coded for the content of the utterances based on whether they promoted the saving or sacrificing a target, and also the relative strength of the utterance i.e. whether it was produced in an absolute manner (strong) or whether it was expressed as a question or with a condition (weak). Finally, again during the middle phase of the task, we coded the responsive utterances, where one of the participants reacts to a suggestion made previously (e.g., "Yes that's true, and we don't even know if the medicine works"). Similarly to the initiating utterances, we first coded the content of the responsive utterances based on whether they promoted the saving or sacrificing a target. Furthermore, we coded for the interactional pattern based on whether the responsive utterance supported what the co-participant had said previously (agreement) or was in opposition to what the co-participant had said (disagreement). The responsive turns' annotations as agreeing or disagreeing were determined based on the participants' own orientations: we examined if and how the responsive turn was interactionally produced as (dis)agreeing with the prior. Responsive utterances that neither clearly 
supported nor opposed the prior were considered as ambiguous and excluded from the analysis.

Six dyads (i.e., $20 \%$ of the entire data set) were randomly chosen to be independently annotated by a second rater for validation. Cohen's kappa coefficient was chosen as the statistical measure of interrater reliability. The derived kappa coefficient of 0.77 suggests a substantial amount of agreement (Landis and Koch, 1977), but it should be noted that this does not account for missing cases (approximately $37 \%$ of all annotations) where one rater had no comparable annotation at the spot.

In respect to the SC and gaze analysis, each annotated initiating and responsive utterance was regarded as a $4 \mathrm{~s}$ segment, beginning from $2 \mathrm{~s}$ before the point of annotation and ending $2 \mathrm{~s}$ after. This was chosen to accommodate the fact that there's no clear singular point in time when the participants 'should' physiologically react and that SC responses in particular can have a several second latency from the onset of a stimuli (Dawson et al., 2017).

\section{Skin Conductance Responses}

The SC signal was deconvoluted with the Richardson-Lucy algorithm (Richardson, 1972) in order to distinguish between overlapping SC responses (Bach et al., 2010; Benedek and Kaernbach, 2010). SC responses were identified (see Figure 1) from the deconvoluted signal through peak detection - all local maxima with a minimum prominence of $0.05 \mu \mathrm{S}$ and a height of one standard deviation or higher above the mean level.

\section{Face Detection}

We used the YOLOv3 (Redmon and Farhadi, 2019) object detection algorithm (open-source implementation from: https:/github.com/sthanhng/yoloface) to detect faces in each video frame (videos were produced by the forward cameras of the eye-trackers). Correspondingly, the gaze of each participant was determined using the $3 \mathrm{D}$ calibration and mapping mode of the Pupil Capture software. The gaze signal and the detected face locations were used to determine whether each participant was gazing the other or not on each frame (i.e. whether their gaze was located within the detected face).

In respect to the annotated segments, we determined a gazing rate for each participant by dividing the number of frames where the participant was gazing at their partner by the total number of frames.

\section{RESULTS}

The results section is divided into seven subsections. The first subsection investigates Hypotheses 1 and $3 c$, specifically the SC rates during the three main phases (beginning, middle, end) of the conversation. The next three sections concern Hypotheses 2 and 3b, and describe variables influencing, or at least correlating with, the gazing patterns during initiating and responsive utterances and the amount of time between these utterances. The two following sections feature the SC rates during initiating and responsive utterances in a similar manner. The final subsection concerns the general contents and patterns of talk concerning Hypothesis 3 and the differences between participants with and without a depression diagnosis.

Unless otherwise specified, statistical analyses were conducted via generalized linear mixed models (GLMM) with Gaussian response and identity link to control for the nonindependence of measures from individual dyads and participants. The $p$-values for GLMMs were estimated with the Satterthwaite approximation (Satterthwaite, 1941).

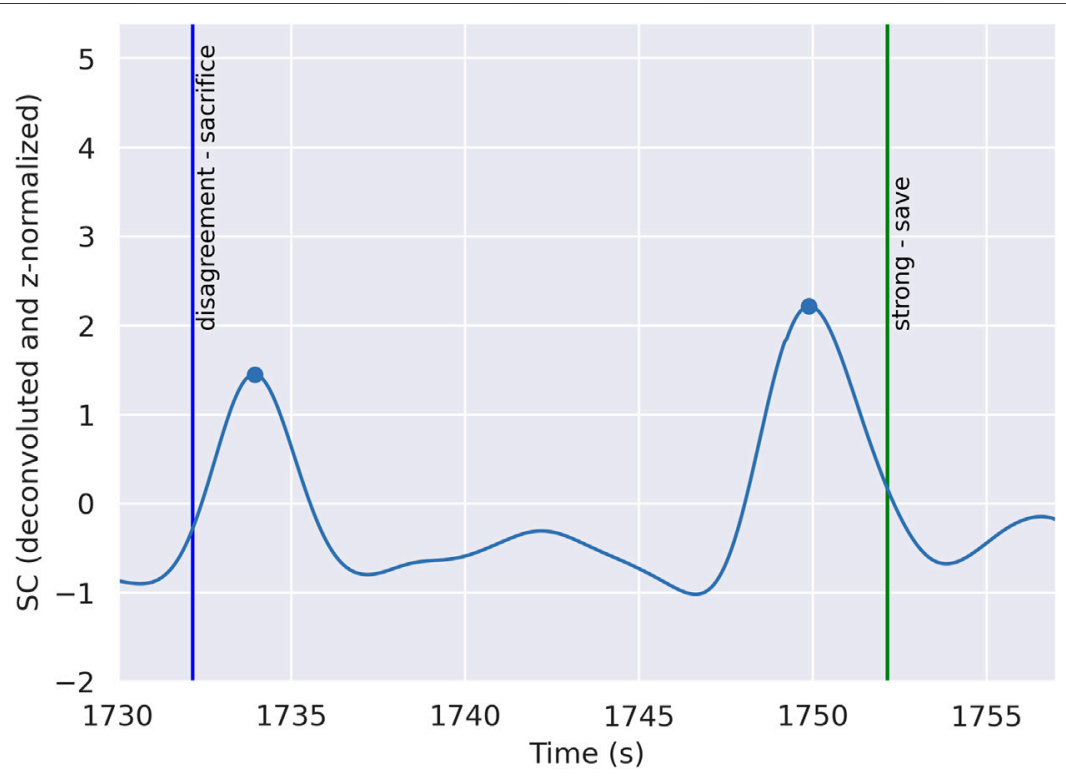

FIGURE 1 | A sample time series of a deconvoluted, z-normalized SC signal (depicted by the blue line). The blue dots indicate the peaks of individual SC responses. The blue vertical line indicates a responsive utterance and the green line an initiating utterance from the participant whose SC signal is depicted in the figure. 


\section{Skin Conductance Response Rates in Different Phases of Conversation}

In the investigation of Hypotheses 1 and $3 c$, we examined the three annotated phases of the conversation to see if mean SC response rates differed between the phase or on the basis of whether the participants were diagnosed with depression or not. Unlike in the other analyses where the utterances are always regarded as 4-s segments, the phases were of varying length (see Supplementary Materials for details on how the length of the phases were determined).

The statistical analysis was conducted via a GLMM. Depression diagnosis and phase (three levels: beginning, middle, end) were included as fixed effects. The dyad and participant were incorporated as nested random effects (random intercepts) to control for the non-independence of participants within a dyad and repeated measures from an individual participant. The model summary can be seen on Table 1 .

In contrast to the middle phase, both the beginning phase ( $p=$ $0.02)$ and end phase $(p<0.001)$ had significantly higher SC response rates as predicted by the hypothesis. The depression diagnosis of the participants, however, had no significant effect. The mean response rates in different phases are visualized in Figure 2.

\section{Gaze Patterns During Initiating Utterances}

To investigate Hypotheses $2 \mathrm{a}, 2 \mathrm{~b}$ and $3 \mathrm{~b}$ in terms of the participants' gaze behavior, we examined the gazing ratios of speakers of initiating utterances in respect to whether the utterance was strong or weak, whether they proposed to sacrifice or save one of the passengers, and whether the speaker was diagnosed with depression or not. As explained in the Methods section, each utterance was regarded as a $4 \mathrm{~s}$ segment for the purpose of the analysis.

In the GLMM analysis, diagnosis status of the speaker, content (sacrifice or save), and strength (weak or strong) of the utterance were chosen as
TABLE 1 | Coefficient table to probe the effect of the depression diagnosis and phase of the conversation (the middle phase was chosen as the baseline category) on SC response rates. The marginal $R^{2}$ for the model was 0.10 and the conditional $R^{2}$ was 0.19 .

\begin{tabular}{lccccc} 
& Estimate & Std. Error & df & $\boldsymbol{t}$ value & $\operatorname{Pr}(>|\mathbf{t}|)$ \\
\hline (Intercept) & 3.3124 & 0.5764 & 53.8627 & 5.7472 & $4.724 \mathrm{E}-08^{\star * \star}$ \\
Diagnosis & 0.3708 & 0.8104 & 58.2086 & 0.4575 & 0.649 \\
Phase_Beginning & 1.7389 & 0.7523 & 114.9546 & 2.3113 & $0.02266^{*}$ \\
Phase_End & 3.2784 & 0.7225 & 110.1424 & 4.5379 & $1.456 \mathrm{E}-05^{\text {** }}$
\end{tabular}

fixed effects with the content ${ }^{\star}$ strength interaction term included. As previously, the dyad and participant were incorporated as nested random effects. The model summary can be seen on Table 2 .

Depression diagnosis had a significant negative effect ( $p=$ 0.024 ) on gazing, indicating that depressed participants gazed at their co-participant less than the non-depressed participants as predicted in the hypotheses. On the other hand, the main effects of content and strength were not significant. However, the content $^{\star}$ strength interaction term had a positive significant effect $(p=0.043)$, indicating that strong initiating utterances had a more positive effect on gazing when the proposal was to save someone. The mean gazing ratios in respect to the depression diagnosis of the speaker, strength and content are visualized in Figure 3.

\section{Gaze Patterns during Responsive Utterances}

Similarly, to probe Hypotheses 2a, 2d and 3b, we investigated gazing ratios during responsive utterances in respect to the responder's depression diagnosis status, whether the responsive utterance aligned with what their co-participants

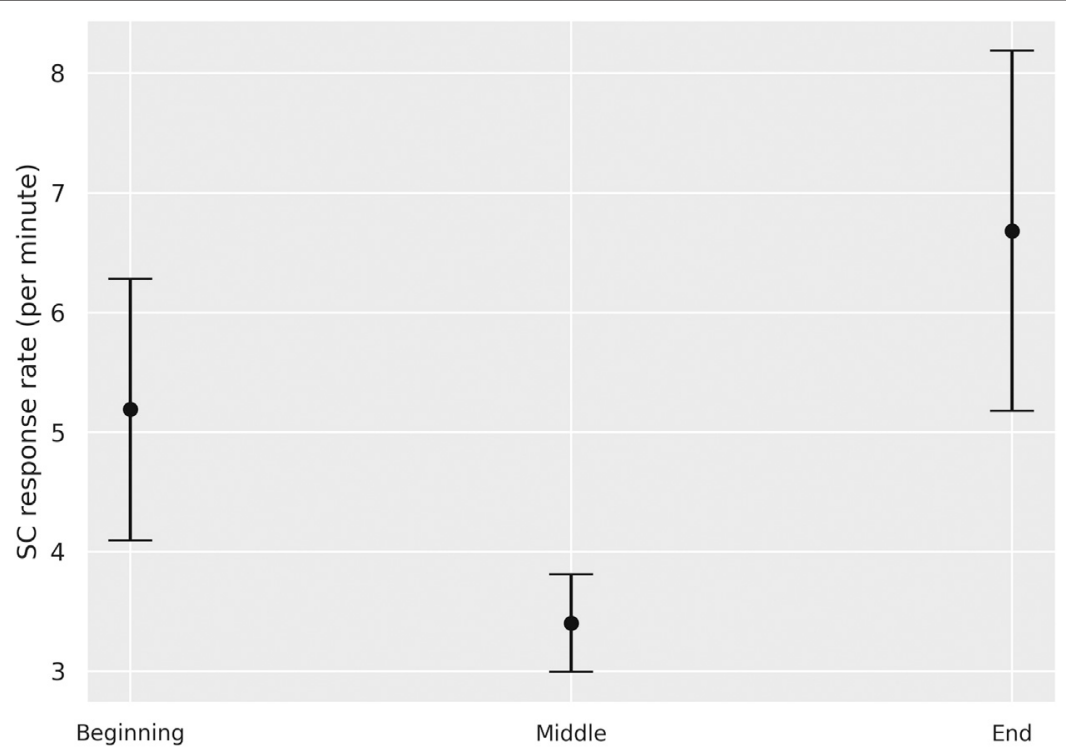

FIGURE 2 | Mean SC response rates and 95\% confidence intervals in the beginning, middle and end phase. The middle phase had significantly lower SC response rates than the beginning and end phase. 
TABLE 2 | Coefficient table of the model used to probe the effect of depression diagnosis (Diagnosis; 0 = no depression diagnosis, 1 = depression diagnosis), content (Content; 0 = sacrifice, 1 = save), and strength (Strength; 0 = weak, 1 = strong) on gazing during initiating utterances. The marginal $R^{2}$ (Nakagawa and Schielzeth, 2013) for the model was 0.09 while the conditional $R^{2}$ was 0.58 , indicating that the fixed effects explained $9 \%$ of the variation.

\begin{tabular}{lccccc} 
& Estimate & Std. Error & df & t value & $\operatorname{Pr}(>|\mathbf{t}|)$ \\
\hline (Intercept) & 0.642 & 0.0623 & 84.4635 & 10.3009 & $1.369 \mathrm{E}-16^{\star \star}$ \\
Diagnosis & -0.2373 & 0.1019 & 46.955 & -2.3285 & $0.02424^{*}$ \\
Content & -0.0855 & 0.0696 & 98.5494 & -1.2293 & 0.2219 \\
Strength & -0.0481 & 0.0613 & 107.0032 & -0.785 & 0.4342 \\
Content:Strength & 0.1945 & 0.0949 & 98.6213 & 2.0493 & $0.04309^{*}$
\end{tabular}

had suggested previously and whether the utterance was in favour of sacrificing or saving one of the passengers.

In a comparable manner to how the initiating utterances were examined, the gaze behavior analysis was conducted via a GLMM with diagnosis status of the speaker, agreement (disagreeing or agreeing), and content (sacrifice or save) of the utterance as fixed effects. The model summary can be seen on Table 3. No significant effects were found.

\section{Response Time Patterns}

In respect to Hypothesis 2c, we investigated if there was a correlation between the time interval between initiating utterances and responses and how much the speaker of the initiating utterance had gazed at their co-participant. In addition, we examined if there might be an effect regarding response times in terms of how strong the initiating utterance was, the depression status of the participants, and whether the responsive utterance aligned with the original proposal. The summary of the constructed GLMM can be seen on Table 4.

Both the gazing ratio of the speaker of the initiating utterance $(p=$ $0.02)$ and the agreement of the response $(p=0.002)$ had a significant negative effect on the response time. In other words, higher amount of gaze (by the speaker of the initiating utterance) and agreeing responsive utterances were associated with faster responses.

\section{Skin Conductance Response Rates During Initiating Utterances}

Comparably to the gaze analysis, in investigation of Hypotheses $2 \mathrm{a}, 2 \mathrm{~b}$ and $3 \mathrm{c}$ in respect to skin conductance, we examined the SC response rates of the participants during initiating utterances in terms of whether the utterance was strong or weak, whether the proposal was to sacrifice or save one of the passengers, and whether the speaker was diagnosed with depression or not. As before, each initiating utterance was regarded as a $4 \mathrm{~s}$ segment.

As in the gaze pattern analysis, diagnosis, strength, content and the strength* ${ }^{*}$ content interaction were included as fixed effects in the GLMM. Dyad and participant were chosen as nested random effects. The model summary can be seen on Table 5.

No statistically significant effects were found. However, it may be worth noting that the magnitudes of the estimated coefficients of strength and content are relatively large.
TABLE 3 | Coefficient table of the model used to probe the effect of depression diagnosis (Diagnosis; 0 = no depression diagnosis, 1 = depression diagnosis), content (Content; 0 = sacrifice, 1 = save), and agreement (Agreement; 0 = disagreement, 1 = agreement) on gazing during responsive utterances. The marginal $R^{2}$ for the model was 0.02 and the conditional $R^{2}$ was 0.69 .

\begin{tabular}{lccccc}
\hline & Estimate & Std. Error & df & $\boldsymbol{t}$ value & $\operatorname{Pr}(>\mid \mathbf{t})$ \\
\hline (Intercept) & 0.6223 & 0.0757 & 144.5104 & 8.2248 & $\begin{array}{c}1.033 \mathrm{E}- \\
13^{\star \star \star}\end{array}$ \\
& & & & & 0.4114 \\
Diagnosis & -0.0852 & 0.1029 & 54.4076 & -0.8279 & 0.09121 \\
Agreement & -0.1088 & 0.064 & 142.5186 & -1.7006 & 0.0912 \\
Content & -0.0314 & 0.0702 & 139.5484 & -0.4467 & 0.6558 \\
Agreement! & 0.1297 & 0.0877 & 145.6609 & 1.4783 & 0.1415 \\
Content & & & & &
\end{tabular}

\section{Skin Conductance Response Rates during Responsive Utterances}

To probe Hypotheses 2a, 2d and 3c, SC response rates during responsive utterances were investigated in a comparable manner. As in the gaze analysis, diagnosis, agreement (disagreeing or agreeing), and content (sacrifice or save) of the utterance were chosen as fixed effects for the GLMM (see Table 6).

Both agreement $(p=0.034)$ and content $(p=0.023)$ had a negative significant effect, indicating that both disagreeing and sacrificing responsive utterances were associated with higher SC response rates compared to agreeing and saving utterances. This is in line with our hypotheses. The mean SC response rates in respect to agreement and content of the utterances are visualized in Figure 4.

\section{General Contents and Patterns of Talk}

In total, each participant made on average $2.3(\mathrm{SD}=1.8)$ initiating utterances (see Table 7 for relative frequencies in respect to the different classifications). There was no significant difference between participants with or without depression (participants with depression made on average 2.2 initiating utterances vs. 2.33 by participants without depression. Wilcoxon signed rank test for the case pairs, $p=0.55$ ). Nor was there a large difference among depressed and non-depressed participants in the proportion of initiating utterances ( $70 \%$ among participants with depressions vs $60 \%$ among those without. Wilcoxon signed rank test for the case pairs, $p=0.24$ ) that were to sacrifice one of the passengers as

TABLE 4 | Coefficient table of the model used to probe the effect of the gazing ratio of the initiating utterance for the speaker (Gazing_Ratio_IU ), the depression diagnosis of the speaker of the initiating utterances (Diagnosis_IU) and the speaker of the responsive utterance (Diagnosis_RU), strength of the original initiating utterance (Strength_IU; 0 = weak, 1 = strong), and agreement of the responsive utterance (Agreement_RU; 0 = disagreement, 1 = agreement) on how long (in seconds) it takes for a response to take place. The marginal $R^{2}$ for the model was 0.17 and the conditional $R^{2}$ was 0.35 .

\begin{tabular}{lccccc} 
& Estimate & Std. Error & df & $\boldsymbol{t}$ value & $\operatorname{Pr}(>|\mathbf{t}|)$ \\
\hline (Intercept) & 29.4291 & 5.0662 & 62.3832 & 5.8089 & $2.297 \mathrm{E}-07^{\star \star \star}$ \\
Gaze_IU & -10.5804 & 4.3668 & 67.2887 & -2.4229 & $0.01809^{\star}$ \\
Strength_IU & -2.0817 & 2.8222 & 63.2213 & -0.7376 & 0.4635 \\
Agreement_RU & -11.2896 & 3.5027 & 64.7889 & -3.2231 & $0.001987^{\star \star}$ \\
Diagnosis_IU & -2.6507 & 4.3077 & 36.2257 & -0.6153 & 0.5422 \\
Diagnosis_RU & -3.3169 & 4.1973 & 38.8602 & -0.7903 & 0.4342
\end{tabular}


TABLE 5 | Coefficient table of the model used to probe the effect of depression diagnosis (Diagnosis; 0 = no depression diagnosis, 1 = depression diagnosis), content (Content; 0 = sacrifice, 1 = save), and strength (Strength; 0 = weak, 1 = strong) on skin conductance rates during initiating utterances. The marginal $R^{2}$ for the model was 0.05 and the conditional $R^{2}$ was 0.20 .

\begin{tabular}{lccccc} 
& Estimate & Std. Error & df & $\boldsymbol{t}$ value & $\operatorname{Pr}(>|\mathbf{t}|)$ \\
\hline (Intercept) & 5.1837 & 1.2342 & 64.6334 & 4.2001 & $8.339 \mathrm{E}-05^{\star \star \star}$ \\
Diagnosis & -1.3089 & 1.5256 & 100.2212 & -0.858 & 0.393 \\
Content & -2.3385 & 1.7294 & 118.9236 & -1.3522 & 0.1789 \\
Strength & 2.2376 & 1.4767 & 123.6218 & 1.5153 & 0.1323 \\
Content:Strength & -0.2716 & 2.38 & 117.9644 & 0.1141 & 0.9093 \\
\hline
\end{tabular}

opposed to saving them. There was no difference in terms of the strength of the utterance either (approximately $50 \%$ of the utterances were strong among both participants with and without depression. Wilcoxon signed test for the case pairs, $p=0.96)$.

No differences were found in the patterns of the responsive utterances (see Table 8 for relative frequencies) either in respect to participants' diagnostic status - approximately $70 \%$ of the responses were agreeing for both groups (Wilcoxon signed test for the case pairs, $p=0.39$ ).

In terms of the final choice on who should be jump, the balloon pilot was chosen by 16 dyads (case pairs: 8 , control pairs: 8 ), the cancer scientist by 12 dyads (case pairs: 6, control pairs: 6) and the pregnant primary school teacher by two dyads (case pairs: 1 , control pairs: 1 ). The two distributions between control and case pairs were identical.

In summary, in terms of the general contents and patterns of talk we observed none of the effects (in respect to the depression diagnosis of the participants) that were predicted by Hypothesis 3 .

\section{DISCUSSION}

In this study, we have considered how the participants' interactional behavior during a dyadic moral dilemma task is reflected in their psychophysiological responses and gaze behavior. Here we will discuss the results in relation to our specific research hypotheses.

Our Hypothesis 1 was informed by the assumption that transitions between activities necessitate "heightened intersubjectivity", which can show in the participants' bodies as higher psychophysiological arousal during the beginning and end phases of the conversational task than during the middle phases of the task (see Stevanovic et al., 2017). Our data of the

TABLE 6 | Coefficient table of the model used to probe the effect of depression diagnosis (Diagnosis; 0 = no depression diagnosis, 1 = depression diagnosis), content (Content; 0 = sacrifice, 1 = save), and agreement (Agreement; 0 = disagreement, 1 = agreement) on skin conductance rates during responsive utterances. The marginal $R^{2}$ for the model was 0.06 while the conditional $R^{2}$ was 0.15 , indicating that the fixed effects explained $6 \%$ of the variation.

\begin{tabular}{lccccc}
\hline & Estimate & Std. Error & df & $\boldsymbol{t}$ value & $\operatorname{Pr}(>\mid \mathbf{t})$ \\
\hline (Intercept) & 8.2859 & 1.5796 & 151.1039 & 5.2456 & $5.177 \mathrm{E}-07^{\star \star \star}$ \\
Diagnosis & -1.2827 & 1.1765 & 163.4066 & -1.0902 & 0.2772 \\
Agreement & -3.5819 & 1.68 & 179.8155 & -2.1321 & $0.03435^{\star}$ \\
Content & -4.294 & 1.8698 & 176.8382 & -2.2965 & $0.02282^{\star}$ \\
Agreement:Content & 2.5064 & 2.2251 & 179.5985 & 1.1265 & 0.2615
\end{tabular}

TABLE 7 | Relative frequency of initiating utterances made by depressed $(N=15)$ and non-depressed $(N=45)$ participants in respect to their strength (strong or weak) and content (sacrifice or save).

\begin{tabular}{lcc}
\hline & Depression diagnosis & \\
\hline & Strong & Weak \\
\hline Save & $18 \%$ & $12 \%$ \\
Sacrifice & $30 \%$ & $39 \%$ \\
\hline & No diagnosis & \\
& Strong & Weak \\
\hline Save & $22 \%$ & $18 \%$ \\
Sacrifice & $29 \%$ & $31 \%$ \\
\hline
\end{tabular}

particicipants' SC response rates clearly support that conclusion. It is during the beginning and end phases of the conversational task that the participants need to pay particular attention to each other and determine how to start the decision-making activity and bring it coordinatedly to a close. In addition to reaching a common understanding of what the actual and binding decision ultimately is, the participants also need to manage their interaction then and there and know when it is appropriate to move on.

Next, we assumed that, during the middle phase of the moral dilemma task, the content of talk and the patterns of gaze and the turn-by-turn unfolding of conversational utterances are reflected in the participants' psychophysiological responses. With regard to the SC response rates (Hypothesis 2a), we hypothesized that utterances which suggest a balloon passenger to sacrifice him- or herself by jumping from the balloon will be associated with higher SC response rates in the speaker than the utterances concerning the saving of a balloon passenger. Our results support this conclusion. While making a conversational contribution in itself always entails speakers to put something of him- or herself "out there" for others to judge, and thus to submit into a vulnerable position (see Goffman, 1959; Goffman, 1967), our results show that the specific contents of utterances play a significant role in how they are psychophysiologically underpinned. Our results suggest that, in the context of solving a moral dilemma, suggesting a

TABLE 8 | Relative frequency of responses made by depressed $(N=15)$ and nondepressed participants $(N=45)$ in respect to their agreement (disagreeing or agreeing) and content (sacrifice or save).

\begin{tabular}{lcc}
\hline & Depression diagnosis & \\
\hline & Agreeing & Disagreeing \\
\hline Save & $37 \%$ & $15 \%$ \\
Sacrifice & $35 \%$ & $13 \%$ \\
\hline & No diagnosis & \\
& Agreeing & Disagreeing \\
Save & $22 \%$ & $19 \%$ \\
Sacrifice & $47 \%$ & $11 \%$
\end{tabular}




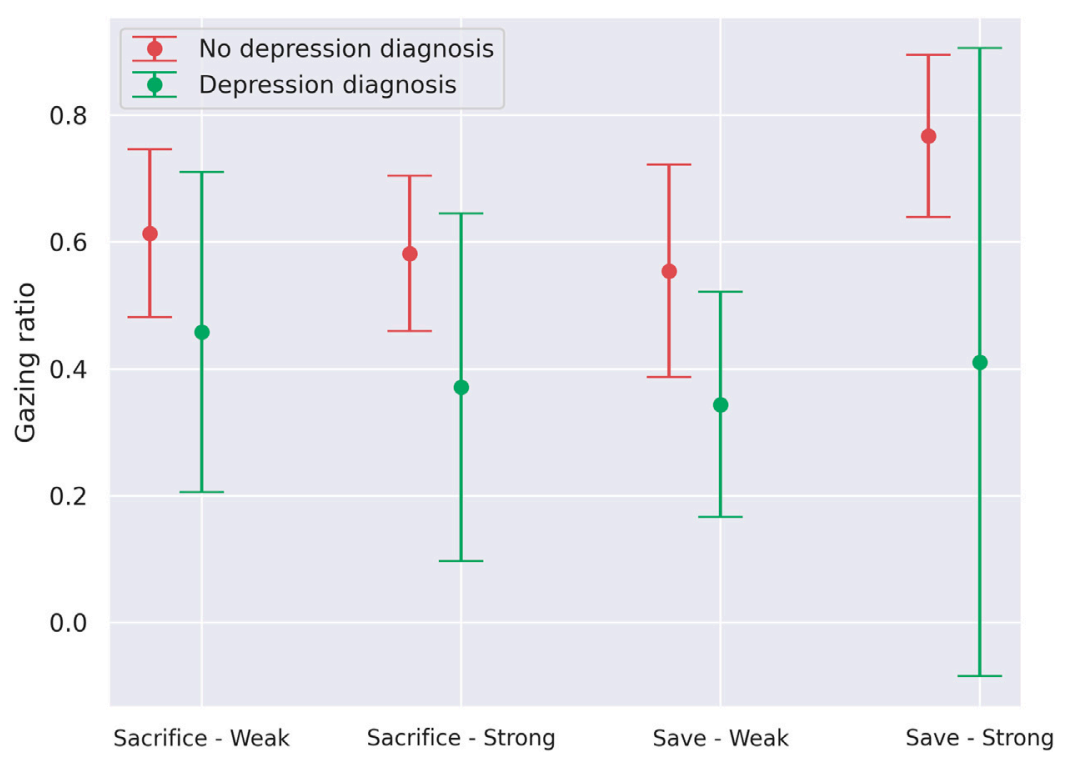

FIGURE 3 | Mean gazing ratios and 95\% confidence intervals during initiating utterances both in respect to their strength (weak or strong) and content (sacrifice or save) and whether the speaker had a depression diagnosis (green bars) or not (red bars).

person (pregnant woman, scientist or balloon pilot) to sacrifice themselves can be experienced as an interactionally more risky or threatening move than suggesting that someone should be saved. Notably, however, our results on the proportionately higher SC response rates in the sacrificing utterances are differentiated depending on the status of the utterance as an initiating vs. responsive one. The observed higher SC response rates were statistically significant only with regard to the responsive utterances, while the effect was smaller in the initiating utterances. At this point, we may only speculate why this might be the case. One possibility is that, in this particular context, responsive sacrificing utterances are one step closer to the reaching of the final moral decision (of who should jump from the balloon), which might show in an elevated SC response in the producer of the responsive utterance.

We hypothesized that strong initial utterances are associated with more gazing towards the co-participant than the weak ones

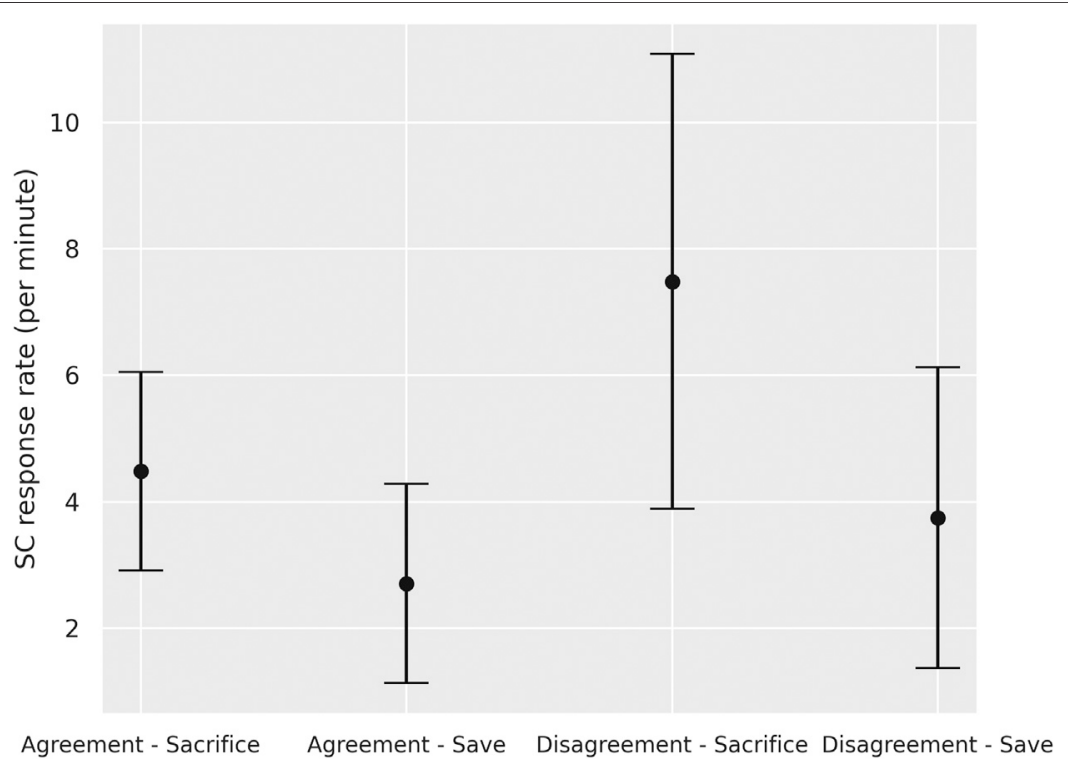

FIGURE 4 | Mean SC response rates and 95\% confidence intervals during responsive utterances in respect to whether they were agreeing or disagreeing, and to save or sacrifice. Both saving and agreeing had a negative effect in respect to SC response rate. 
(Hypothesis 2b). Our eye tracking results support this conclusion only partially: the initiating utterances exhibited an interaction effect between the relative strength of the verbal expression (e.g., "should we spare the woman?" vs. "the pregnant woman cannot possibly jump") and the content of the expression (saving vs. sacrificing a person), with partner gazing occurring most frequently during those utterances in which the speaker argued strongly for saving a person. In other words, the effect was not observable in strong utterances where a person was suggested to be sacrificed (e.g., "we don't need the pilot"). Prior literature has pointed to the function of gaze as a way to increase the strength of one's utterance, which gets support from our result of more partner gazing leading to faster co-participant responses (Hypothesis 2c). This is linked to what Stivers and Rossano (2010) have described as "mobilizing response": gaze increases the pressure on the recipient to respond to an utterance. This is also in line with the notions in psychological literature according to which gaze directed straight to the co-participant is perceived as more dominant than gaze withdrawal (Argyle and Dean, 1965; Hall et al., 2005). Studies have also shown that the content of the talk affects gazing behavior. In general, there is usually more direct eye contact when the topic under the discussion is more "easy" and cognitively more straightforward (Argyle and Dean, 1965). When discussing difficult topics, feeling uncertain or ashamed, people tend to direct their gaze away from their coparticipant (Burgoon et al., 1996; Bente et al., 1998). This literature is also very much in line with our results, as strong utterances proposing the saving of a person were associated with more gazing towards the co-participant than sacrificing utterances, which can be seen as topically more delicate.

With regard to the patterns of talk, we hypothesized that responsive utterances that express disagreement with the initial proposal are produced with a delay and associated with higher SC response rates in the participant (Hypothesis 2d). This hypothesis was supported by our data. The result can be clarified with reference to the fundamental notion of "preference organization" most famously promoted in the field of conversation analysis (Pomerantz, 1984; Bilmes, 1988). Disagreement in decision-making is often expressed with a delay, which conveys that something problematic is going on, whereas acceptance is done straight away (Houtkoop, 1987; also see; Davidson, 1984; Pomerantz, 1984). Furthermore, in this context of moral stance-taking, displaying agreement with the co-participant's stance can be described as an affiliative action (Stivers, 2008; Stivers et al., 2011; Lindström and Sorjonen, 2013), whereas disagreement conveys disaffiliation. Our results suggest that the problematic interactional experience associated with disagreement and disaffiliation may have a psychophysiological correlate, leading to increased arousal in the speaker. Even though the anxiety-provoking and stressful nature of these disaffiliative actions seems intuitively plausible, the finding is not self-evident. In their study of storytelling interaction, Peräkylä and colleagues (2015) found that it was the empathetic and affiliative displays of recipiency to a story that led to increased psychophysiological arousal, which the authors interpreted with reference to the notion of "emotional labor" (Hochschild, 1979). As an activity, however, storytelling can be considered to be fundamentally different from solving moral dilemmas together. In this context, we argue, agreement, in the sense of going along with the co-participant's proposal, is not specifically a taxing interactional task, whereas disagreeing with the co-participant's moral stance might require emotional work (e.g., cautious formulations of disagreement, vigilant monitoring of co-participant reactions) to limit the damage that the disagreement might cause to the solidarity and affiliation between the participants.

Finally, we suggested that the contents and patterns of talk (Hypothesis 3a), patterns of gaze behavior (Hypothesis 3b), and the SC response rates (Hypothesis 3c) may be different for participants with and without depression. As for the contents and patterns of talk, we found no differences between the participant groups. While we assumed that participants with depression diagnosis would make fewer proposals with a higher proportion of sacrificing (vs. saving) proposals than participants without depression, our results did not lend support to such conclusions. As previous studies have shown (Kirk et al., 2000), participants diagnosed with depression can be highly skilled in concealing their condition, which may also have been the case in our sample. Concealing depressive symptoms can be motivated, for example, by a desire to maintain normality in front of other people (Draucker, 2005) and cultural patterns where emotional control, self-esteem, and invulnerability are central virtues (Emslie et al., 2006). We also hypothesized that participants with depression diagnosis would differ in their psychophysiological responses to conversational phenomena, but this prediction was not supported by our data. Our results therefore cannot shed light on the mixed evidence so far, involving both the ideas of the physiological underarousal (Grossberg, 1972; Benning and Ait Oumeziane, 2017) and increased threat arousal and worry (Starcevic, 1995; Stevanovic et al., under review) as parts of depressive symptomatology. However, we did find differences in the patterns of gaze behavior between the groups of depressed and non-depressed participants. In line with our hypothesis, the participants with depression were gazing less towards their co-participants, this result being statistically significant specifically during the production of initiating utterances, which may be argued to be most critical utterances in determining which direction the conversation will take. Hence, as all interactional resources, also gaze behaviors have distinct consequences depending on their precise location within interactional sequences (Rossano, 2012), which means that also interactional deficits should be examined by bearing in mind that it is specifically during those moments where partner gazing is most critical that also a lack of gaze may have quite drastic interactional corollaries (Wiklund 2012).

Our study has at least five key limitations, which we will discuss below. First, all the participants in our study were female. If our sample had included male dyads or crossgender dyads, the results might have been different. For example, Tang and Schmeichel (2015) found that direct eye contact with a target face especially affected the men's behavior, who acted in a more dominant fashion when making decisions in a hypothetical ultimatum game. Second, the fact that the participants were strangers to each other may have generated different results from what dyadic interactions between everyday acquaintances, friends, or family members would have brought about. Third, the participant sample for this research consists of volunteers, which can lead to a selfselection bias. As the participants were socially courageous 
enough to decide to volunteer in a study where one is expected to be talking with a stranger, it is likely that those who find such situations particularly stressful did not take part in our study, which may have influenced the results concerning stress-related physiological responses. Fourth, it should be noted that, though skin conductance measures provide powerful tools for assessing the level of arousal in participants, they provide no direct information about the valence of that arousal. Finally, the methodology we have utilized to investigate the psychophysiological experience of individuals with depression is somewhat limited and it should be complemented by other methods, such as in-depth studies of their everyday living environment, to reach a more comprehensive picture about their interactional competences and experiences in solving moral dilemmas with other people. In addition, the interdisciplinarity of our approach is associated with a set of theoretical and methodological contradictions that call for commenting. As pointed out at the beginning of this paper, conversation analysis is essentially about investigating naturally-occurring interactions in order to identify the participants' own orientations to interactional patterns and events (see e.g., Garfinkel and Harvey, 1970: 345; Schegloff, 1997). Our study involved two different types of compromises in this regard. First, our investigation of the psychophysiological underpinnings of interaction was conducted in a laboratory environment, where the realization of the interaction was under the control of the researchers. Our study was, however, informed by previous case-by-case conversation analytic studies on joint decision-making, proposals, and agreements (e.g., Davidson, 1984, Pomerantz, 1984; Stevanovic, 2013; Stevanovic, 2015) and we sought to design our task instructions to maintain the essential natural dynamics of these particular interactional phenomena as far as possible. Second, the relatively high level of "noise" that is an inevitable part of psychophysiological signal made it necessary for us to resort to coding and quantification and thus to go beyond the case-by-case qualitative analysis of interactional sequences, where the focus is on how the meaning of each behavior is collaboratively negotiated throughout the sequence. While our coding of "strong" and "weak" proposal forms was thus essentially a matter of applying previous conversation-analytic findings on this dataset in an $a$ priori fashion, as for agreements and disagreements, in contrast, the participants' own orientations were incorporated in our coding scheme. From this point of view, we believe that our findings are not entirely foreign to the emic concerns that conversation analysts are generally interested in.

Earlier psychological studies investigating moral dilemmas have either focused on the individual and his/her moral judgment (e.g., Christensen et al., 2014) or, in contrast, studied the interactional patterns that the task generates without reference to the specific content of the conversational actions (e.g., Lavelle et al., 2014). This same tension can be found more generally in different social psychological domains, where the main focus is often in either the content of talk (e.g., Bales, 1950) or in the patterns of the turn-by-turn unfolding of interaction (e.g., Schegloff, 2007). Our study has shown that both of these aspects of human interaction are highly relevant, as they resonate in the participants' physical bodies. Furthermore, our research can contribute to the field of studying human moral judgment. Studies utilizing hypothetical moral dilemmas have been criticized for having little predictive value for actual behavior (see Bostyn et al., 2018), as participants in real-life situations refer to their "commonsense morality" (Kahane, 2015) instead of following purely deontological or utilitarian rules. What the discussion has been lacking, however, is the fact that moral decisions in the real world are rarely mulled over in solitude. Instead, people tend to discuss their dilemmatic situations and the different choices that they entail with other people and when they do, our study suggests that the contents and patterns of their interactional contributions reverberate in their physical bodies. Thus, to increase understanding of how moral decisions, which may sometimes have profound consequences, come to being, we need a deeper understanding of the affective and psychophysiological processes that underlie social situations.

\section{DATA AVAILABILITY STATEMENT}

The raw data supporting the conclusions of this article will be made available by the authors, without undue reservation.

\section{ETHICS STATEMENT}

The studies involving human participants were reviewed and approved by Ethics Committee of the Helsinki University Central Hospital [18.06.2018]. The patients/participants provided their written informed consent to participate in this study.

\section{AUTHOR CONTRIBUTIONS}

EK is the main author of the paper and together with ST and MS was responsible for the analysis and interpretation of the results. ST was responsible for data collection and ES for the verification of the participants' depression diagnoses. Experiments were run by ST, KV and MJ. The writing of the first draft was accomplished by EK, MS, ST, TV and EW, and the revised version by EK, MS, ST and TV. MS was responsible for the conception and design of the study. All authors have approved the submitted manuscript and agreed to be personally accountable for their own contributions and for the integrity of the study.

\section{FUNDING}

This work was supported by the Academy of Finland (grant number 307630) and the University of Helsinki. 


\section{REFERENCES}

Aguilar, P., Brussino, S., and Fernández-Dols, J.-M. (2013). Psychological distance increases uncompromising consequentialism. J. Exp. Soc. Psychol. 49 (3), 449-452. doi:10.1016/j.jesp.2013.01.002

Al-Mosaiwi, M., and Johnstone, T. (2018). In an absolute state: elevated use of absolutist words is a marker specific to anxiety, depression, and suicidal ideation. Clin. Psychol. Sci. 6, 529-542. doi:10.1177/ 2167702617747074

Allen, N. B., Gilbert, P., and Semedar, A. (2004). "Depressed mood as an interpersonal strategy: the importance of relational models," in Relational Models Theory: A Contemporary Overview, Editor N. Haslam (Mahwah, NJ: Lawrence Erlbaum), 309-334.

American Psychiatric Association (2013). Diagnostic and statistical manual of mental disorders. 5th ed. Washington, DC: American Psychiatric Association.

Amit, E., and Greene, J. D. (2012). You see, the ends don't justify the means: visual imagery and moral judgment. Psychol. Sci. 23 (8), 861. doi:10.1177/ 0956797611434965

Angus, L. E., and Greenberg, L. S. (2011). Working with narrative in emotionfocused therapy: changing stories, healing lives. Washington, DC: American Psychological Association.

Argyle, M., and Dean, J. (1965). Eye-contact, distance and affiliation. Sociometry, 28, 289-304. doi:10.2307/2786027

Arundale, R. B. (2020). Communicating and relating: constituting face in everyday interacting. New York: Oxford University Press.

Atkinson, J. M., and Heritage, J. (1984). Structures of Social Action: Studies in Conversation Analysis. Cambridge, UK: Cambridge University Press.

Bach, D. R., Flandin, G., Friston, K. J., and Dolan, R. J. (2010). Modelling eventrelated skin conductance responses. Int. J. Psychophysiol. 75 (3), 349-356. doi:10.1016/j.ijpsycho.2010.01.005

Bales, R. F. (1950). Interaction process analysis. Cambridge: Addison-Wesley.

Beauchaine, T. P. (2015). Respiratory sinus arrhythmia: a transdiagnostic biomarker of emotion dysregulation and psychopathology. Curr. Opin. Psychol. 3, 43-47. doi:10.1016/j.copsyc.2015.01.017

Beck, A. T., Ward, C. H., Mendelson, M., Mock, J., and Erbaugh, J. (1961). An inventory for measuring depression. Arch. Gen. Psychiatry 4, 561-571. doi:10. 1001/archpsyc.1961.01710120031004

Beck, A. T. (1979). Cognitive therapy and the emotional disorders. London, England: Penguin.

Benedek, M., and Kaernbach, C. (2010). A continuous measure of phasic electrodermal activity. J. Neurosci. Methods 190 (1), 80-91. doi:10.1016/j. jneumeth.2010.04.028

Benning, S. D., and Ait Oumeziane, B. (2017). Reduced positive emotion and underarousal are uniquely associated with subclinical depression symptoms: evidence from psychophysiology, self-report, and symptom clusters. Psychophysiology 54 (7), 1010-1030. doi:10.1111/psyp.12853

Bente, G., Donaghy, W. C., and Suwelack, D. (1998). Sex differences in body movement and visual attention: an integrated analysis of movement and gaze in mixed-sex dyads. J. Nonverbal Behav. 22 (1), 31-58.

Bilmes, J. (1988). The concept of preference in conversation analysis. Lang. Soc. 17 (2), 161-181. doi:10.1017/S0047404500012744

Boersma, P. (2001). Praat, a system for doing phonetics by computer. Glot International. 5:9/10, 341-345.

Boersma, P., and Weenink, D. (2015). Praat: doing phonetics by computer (v. 6.0.05), Available at: http://www.praat.org.

Bostyn, D. H., Sevenhant, S., and Roets, A. (2018). Of mice, men, and trolleys: hypothetical judgment versus real-life behavior in trolley-style moral dilemmas. Psychol. Sci. 29 (7), 1084-1093. doi:10.1177/0956797617752640

Brown, P., and Levinson, S. (1987). Politeness: some universals in language usage. Cambridge: Cambridge University Press.

Brush, C. J., Olson, R. L., Ehmann, P. J., Bocchine, A. J., Bates, M. E., Buckman, J. F., et al. (2019). Lower resting cardiac autonomic balance in young adults with current major depression. Psychophysiology 56 (8), e13385. doi:10.1111/psyp.13385

Burgoon, J. K., Buller, D. B., and Woodall, W. G. (1996). Nonverbal communication: the un-spoken dialogue. 2nd Ed. New York: McGraw-Hill.

Burns, D. D. (1989). The feeling good handbook: using the new mood therapy in everyday life. New York, NY: William Morrow.
Christensen, J. F., Flexas, A., Calabrese, M., Gut, N. K., and Gomila, A. (2014). Moral judgment reloaded: a moral dilemma validation study. Front. Psychol. 5 , 607. doi:10.3389/fpsyg.2014.00607

Christensen, J. F., and Gomila, A. (2012). Moral dilemmas in cognitive neuroscience of moral decision-making: a principled review. Neurosci. Biobehav. Rev. 36 (4), 1249-1264. doi:10.1016/j.neubiorev.2012.02.008

Davidson, J. A. (1984). "Subsequent versions of invitations, offers, requests, and proposals dealing with potential or actual rejection," in Structures of Social Action: Studies in Conversation Analysis, Editors J. M. Atkinson and J. Heritage (Cambridge, UK: Cambridge University Press), 102-128.

Dawson, M. E., Schell, A. M., and Filion, D. L. (2017). "The electrodermal system," in Cambridge Handbooks in Psychology. Handbook of Psychophysiology, Editors J. T. Cacioppo, L. G. Tassinary, and G. G. Berntson (Cambridge, UK: Cambridge University Press), 217-243.

Dindar, K., Korkiakangas, T., Laitila, A., and Kärnä, E. (2017). An interactional 'live eye tracking' study in autism spectrum disorder: combining qualitative and quantitative approaches in the study of gaze. Qual. Res. Psychol. 14 (3), 239-265. doi:10.1080/14780887.2017.1290174

Draucker, C. B. (2005). Interaction patterns of adolescents with depression and the important adults in their lives. Qual. Health Res. 15, 942-963. doi:10.1177/ 1049732305277859

Edlund, J., House, D., and Beskow, J. (2013). "Gesture movement profiles in dialogues from a Swedish multimodal database of spontaneous speech," in Prosody and Embodiment in Interactional Grammar, Editors P. Bergmann, J. Brenning, M. Pfeiffer, and E. Reber (Berlin, Boston: De Gruyter). doi:10.1515/ 9783110295108

Ekberg, K., and LeCouteur, A. (2015). Clients' resistance to therapists' proposals: managing epistemic and deontic status. J. Pragmat. 90, 12-25. doi:10.1016/j. pragma.2015.10.004

Ellis, T. E., and Rutherford, B. (2008). Cognition and suicide: two decades of progress. Int. J. Cogn. Ther. 1, 47-68. doi:10.1521/ijct.2008.1.1.47

Emslie, C., Ridge, D., Ziebland, S., and Hunt, K. (2006). Men's accounts of depression: reconstructing or resisting hegemonic masculinity? Soc. Sci. Med. 62, 2246-2257. doi:10.1016/j.socscimed.2005.10.017

Ewbank, M. P., Jennings, C., and Calder, A. J. (2009). Why are you angry with me? Facial expressions of threat influence perception of gaze direction. J. Vis. 9 (12), 16-17. doi:10.1167/9.12.16

Fiquer, J. T., Moreno, R. A., Brunoni, A. R., Barros, V. B., Fernandes, F., and Gorenstein, C. (2018). What is the nonverbal communication of depression? Assessing expressive differences between depressive patients and healthy volunteers during clinical interviews. J. Affect. Disord. 238, 636-644. doi:10. 1016/j.jad.2018.05.071

Foot, P. (1967). The problem of abortion and the doctrine of double effect. Oxf. Rev. 5, 5-15.

Garfinkel, H., and Harvey, S. (1970). "On formal structures of practical actions," in Theoretical sociology: Perspectives and developments, Editors J. C. McKinney and E. Tiryakian (New York, NY: Appleton-Century-Crofts, 337-366.

Gilbert, P. (2006). Evolution and depression: issues and implications. Psychol. Med. 36, 287-297. doi:10.1017/S0033291705006112

Goffman, E. (1967). Interaction ritual: essays on face-to-face interaction. Garden City, NY: Doubleday.

Goffman, E. (1955). On face-work; an analysis of ritual elements in social interaction. Psychiatry 18, 213-231. doi:10.1080/00332747.1955.11023008

Goffman, E. (1959). The presentation of self in everyday life. New York: Anchor Books.

Gosling, S. D., Rentfrow, P. J., and Swann, W. B. (2003). A very brief measure of the Big-Five personality domains. J. Res. Pers. 37, 504-528. doi:10.1016/S00926566(03)00046-1

Greene, J., Morelli, S., Lowenberg, K., Nystrom, L., and Cohen, J. (2008). Cognitive load selectively interferes with utilitarian moral judgment. Cognition 107 (3), 1144-1154. doi:10.1016/j.cognition.2007.11.004

Grossberg, S. (1972). A neural theory of punishment and avoidance, II: quantitative theory. Math. Biosci. 15(3), 253-285. doi:10.1016/0025-5564(72)90038-7

Hall, J. A., Coats, E. J., and LeBeau, L. S. (2005). Nonverbal behavior and the vertical dimension of social relations: a meta-analysis. Psychol. Bull. 131, 898-924. doi:10.1037/0033-2909.131.6.898

Henriques, J. B., and Davidson, R. J. (1991). Left frontal hypoactivation in depression. J. Abnorm. Psychol. 100, 535-545. doi:10.1037//0021-843x.100.4.535 
Henriques, J. B., and Davidson, R. J. (1990). Regional brain electrical asymmetries discriminate between previously depressed and healthy control subjects. J. Abnorm. Psychol. 99, 22-31. doi:10.1037//0021-843x.99.1.22

Henriques, J. B., and Davidson, R. J. (2000). Decreased responsiveness to reward in depression. Cogn. Emot. 711-724. doi:10.1080/02699930050117684

Hinchliffe, M., Lancashire, M., and Roberts, F. J. (1970). Eye-contact and depression: a preliminary report. Br. J. Psychiatry 117, 571-572. doi:10.1192/ bjp.117.540.571

Hochschild, A. (1979). Emotion work, feeling rules, and social structure. Am. J. Sociol. 85 (3), 551-575. doi:10.1086/227049

Houtkoop, H. (1987). Establishing agreement: an analysis of proposal-acceptance sequences. Dordrecht, NL: Foris Publications.

Howes, C., Lavelle, M., Healey, P., Hough, J., and McCabe, R. (2016). "Helping hands? Gesture and self-repair in schizophrenia," in Proceedings of LREC 2016 workshop: resources and processing of linguistic and extra-linguistic data from people with various forms of cognitive/psychiatric impairments Portoroź, Slovenia.

Hutchby, I. (1996). Confrontation talk: arguments, asymmetries, and power on talk radio. Mahwah, NJ: Lawrence Erlbaum.

Isaac, L., Vrijsen, J. N., Rinck, M., Speckens, A., and Becker, E. S. (2014). Shorter gaze duration for happy faces in current but not remitted depression: evidence from eye movements. Psychiatry Res. 218 (1-2), 79-86. doi:10.1016/j.psychres.2014.04.002

Jones, I. H., and Pansa, M. (1979). Some nonverbal aspects of depression and schizophrenia occurring during the interview. J. Nerv Ment. Dis. 167, 402-409. doi:10.1097/00005053-197907000-00002

Körner, A., and Volk, S. (2014). Concrete and abstract ways to deontology: cognitive capacity moderates construal level effects on moral judgments. J. Exp. Soc. Psychol. 55, 139-145. doi:10.1016/j.jesp.2014.07.002

Kahane, G. (2015). Sidetracked by trolleys: why sacrificial moral dilemmas tell us little (or nothing) about utilitarian judgment. Soc. Neurosci. 10 (5), 551-560. doi:10.1080/17470919.2015.1023400

Kant, I. (1959). Foundation of the metaphysics of morals. Editors L. W. Beck, Indianapolis: Bobbs-Merrill.

Kellough, J. L., Beevers, C. G., Ellis, A. J., and Wells, T. T. (2008). Time course of selective attention in clinically depressed young adults: an eye tracking study. Behav. Res. Ther. 46, 1238-1243. doi:10.1016/j.brat. 2008.07.004

Kemp, A. H., Quintana, D. S., Gray, M. A., Felmingham, K. L., Brown, K., and Gatt, J. M. (2010). Impact of depression and antidepressant treatment on heart rate variability: a review and meta-analysis. Biol. Psychiatry 67 (11), 1067-1074. doi:10.1016/j.biopsych.2009.12.012

Kendrick, K., and Holler, J. (2017). Gaze direction signals response preference in conversation. Res. Lang. Soc. Interac. 50 (1), 12-32. doi:10.1080/08351813.2017. 1262120

Kirk, L., Haaga, D. A. F., Solomon, A., and Brody, C. (2000). Perception of depression among never-depressed and recovered-depressed people. Cognit. Ther. Res. 24, 585-594. doi:10.1023/A:1005518229707

Koenig, J., Kemp, A. H., Beauchaine, T. P., Thayer, J. F., and Kaess, M. (2016). Depression and resting state heart rate variability in children and adolescents - a systematic review and meta-analysis. Clin. Psychol. Rev. 46, 136-150. doi:10. 1016/j.cpr.2016.04.013

Kohlberg, L. (1969). "Stage and sequence: the cognitive-developmental approach to socialization," in Handbook of Socialization Theory and Research, Editors D. Goslin (Chicago: Rand McNally), 347-480.

Kvalnes, Ø. (2019). Moral reasoning at work. Cham: Palgrave Pivot.

Landis, J. R., and Koch, G. G. (1977). The measurement of observer agreement for categorical data. Biometrics 33, 159-174. doi:10.2307/2529310

Lavelle, M., Healey, P. G., and McCabe, R. (2013). Is nonverbal communication disrupted in interactions involving patients with schizophrenia? Schizophr. Bull. 39, 1150-1158. doi:10.1093/schbul/sbs091

Lavelle, M., Healey, P. G., and McCabe, R. (2014). Participation during first social encounters in schizophrenia. PLoS One 9 (1), e77506. doi:10.1371/journal.pone. 0077506

Lee, J. J., and Gino, F. (2015). Poker-faced morality: concealing emotions leads to utilitarian decision making. Organ. Behav. Hum. Decis. Process. 126. 49-64. doi:10.1016/j.obhdp.2014.10.006

Levenson, R. W., and Gottman, J. (1983). Marital interaction: physiological linkage and affective exchange. J. Pers. Soc. Psychol. 45 (3), 587-597. doi:10.1037//00223514.45.3.587
Lindström, A., and Sorjonen, M.-L. (2013). "Affiliation in conversation," in The Handbook of Conversation Analysis, Editors J. Sidnell and T. Stivers (Malden, MA: Wiley-Blackwell), 250-369.

Luminet, O., Bouts, P., Delie, F., Manstead, A. S. R., and Rimé, B. (2000). Social sharing of emotion following exposure to a negatively valenced situation. Cogn. Emot. 14, 661-688. doi:10.1080/02699930050117666

Markee, N. (2012). "Emic and etic in qualitative research," in The encyclopedia of applied linguistics. New York: Wiley-Blackwell.

McCabe, R., and Lavelle, M. (2012). "Participation in multiparty interaction: a single case analysis of a patient with schizophrenia," in Proceedings of Video Analysis on Work, Interaction and technology: A Festshrift for Christian Heath. (Bayreuth: Landratesaal), 97-108.

Mill, J. S. (1998). Utilitarianism Editors R. Crisp, New York: Oxford University Press.

Montgomery, S., and Åsberg, M. (1979). A new depression scale designed to be sensitive to change. Br. J. Psychiatry 134, 382-389. doi:10.1192/bjp.134.4.382

Moore, A. B., Clark, B. A., and Kane, M. J. (2008). Who shalt not kill? Individual differences in working memory capacity, executive control, and moral judgment. Psychol. Sci. 19 (6), 549-557. doi:10.1111/j.1467-9280.2008.02122.x

Muntigl, P. (2016). "Storytelling, depression, and psychotherapy," in The Palgrave Handbook of Adult Mental Health, Editors M. O'Reilly and J. N. Lester (London: Palgrave Macmillan), 577-596.

Nakagawa, S., and Schielzeth, H. (2013). A general and simple method for obtaining R2 from generalized linear mixed-effects models. Ecol. Evol. 4 (2), 133-142. doi:10.1111/j.2041-210x.2012.00261.x

Needles, D. J., and Abramson, L. Y. (1990). Positive life events, attributional style, and hopefulness: testing a model of recovery from depression. J. Abnorm. Psychol. 99, 156-165. doi:10.1037//0021-843x.99.2.156

Peräkylä, A., Henttonen, P., Voutilainen, L., Kahri, M., Stevanovic, M., Sams, M., et al. (2015). Sharing the emotional load: recipient affiliation calms down the storyteller. Soc. Psychol. Q. 78 (4), 301-323. doi:10.1177/0190272515611054

Pomerantz, A. (1984). "Agreeing and disagreeing with assessments: some features of preferred/dispreferred turn shapes," in Structures of social action: Studies in conversation analysis, Editors J. M. Atkinson and J. Heritage (Cambridge, UK: Cambridge University Press), 57-101.

Purver, M., Healey, P. G. T., King, J., Ginzburg, J., and Mills, G. J. (2003). "Answering clarification questions," in Proceedings of the 4th SIGdial Workshop on Discourse and dialogue (Sapporo, Japan: ACL), 23-33.

Redmon, J., and Farhadi, A. (2019). Yolov3: an incremental improvement. arXiv 2018. arXiv preprint arXiv:1804.02767.

Richardson, W. H. (1972). Bayesian-based iterative method of image restoration. J. Opt. Soc. 62 (1), 55. doi:10.1364/JOSA.62.000055

Rogers, E. S., Ralph, R. O., and Salzer, M. S. (2010). Validating the empowerment scale with a multisite sample of consumers of mental health services. Psychiatr. Serv. 61, 933-936. doi:10.1176/ps.2010.61.9.933

Rossano, F. (2012). Gaze Behavior in face-to-face interaction. $\mathrm{PhD}$ dissertation. Nijmegen: Radboud University.

Rottenberg, J., Clift, A., Bolden, S., and Salomon, K. (2007). RSA fluctuation in major depressive disorder. Psychophysiology 44 (3), 450-458. doi:10.1111/j. 1469-8986.2007.00509.x

Rottenberg, J., Kasch, K. L., Gross, J. J., and Gotlib, I. H. (2002). Sadness and amusement reactivity differentially predict concurrent and prospective functioning in Major Depressive Disorder. Emotion 2, 135-146. doi:10.1037/1528-3542.2.2.135

Rottenberg, J. (2007). Cardiac vagal control in depression: a critical analysis. Biol. Psychol. 74 (2), 200-211. doi:10.1016/j.biopsycho.2005.08.010

Rottenberg, J. (2005). Mood and emotion in major depression. Curr. Dir. Psychol. Sci. 14, 167-170. doi:10.1111/j.0963-7214.2005.00354.x

Rotter, J. (1966). Generalized expectancies for internal versus external control of reinforcement. Psychol. Monogr. 80 (1), 1. doi:10.1037/h0092976

Sanchez, A., Vazquez, C., Marker, C., Lemoult, J., and Joormann, J., (2013). Attentional disengagement predicts stress recovery in depression: an eyetracking study. J. Abnorm. Psychol. 122 (2), 303-313. doi:10.1037/ a0031529

Sarchiapone, M., Gramaglia, C., Iosue, M., Carli, V., Mandelli, L., Serretti, A., et al. (2018). The association between electrodermal activity (EDA), depression and suicidal behaviour: a systematic review and narrative synthesis. BMC psychiatry 18 (1), 22. doi:10.1186/s12888-017-1551-4

Satterthwaite, F. E. (1941). Synthesis of variance. Psychometrika. 6 (5), 309-316. doi:10.1007/BF02288586 
Scheffler, S. (2010). "Morality, and reasonable partiality," in Partiality and Impartiality: Morality, Special Relationships, and the Wider World, Editors B. Feltham and J. Cottingham (Oxford: Oxford University Press).

Schegloff, E. A. (2007). Sequence organization iin interaction: a primer $n$ conversation analysis. Cambridge, UK: Cambridge University Press, 1.

Schegloff, E. A. (1997). Whose text? Whose context? Discourse Soc. 8 (2), 165-187. doi:10.1177/0957926597008002002

Schiffrin, D. (1990). "Management of a co-operative self during argument," in Conflict Talk, Editors A. D. Grimshaw (Cambridge, UK: Cambridge University Press, 241-259.

Schiffrin, D. (1984). Jewish argument as sociability. Lang. Soc. 13 (3), 311-335. doi:10.1017/S0047404500010526

Schulze, L., Renneberg, B., and Lobmaier, J. S. (2013). Gaze perception in social anxiety and social anxiety disorder. Front. Hum. Neurosci. 7, 872. doi:10.3389/ fnhum.2013.00872

Sidnell, J. (2014). "The architecture of intersubjectivity revisited," in The Cambridge Handbook of Linguistic Anthropology, eds. N. Enfield, P. Kockelman, and J. Sidnell (Cambridge: Cambridge University Press), 364-399. doi:10.1017/CBO9781139342872.018

Sloan, D. M., Strauss, M. E., and Wisner, K. L. (2001). Diminished response to pleasant stimuli by depressed women. J. Abnorm. Psychol. 110, 488-493. doi:10. 1037//0021-843x.110.3.488

Smirnov, D., Saarimäki, H., Glerean, E., Hari, R., Sams, M., and Nummenmaa, L. (2019). Emotions amplify speaker-listener neural alignment. Hum. Brain Mapp. 40, 4777-4788. doi:10.1002/hbm.24736

Snyder, M., and Gangestad, S. (1986). On the nature of self-monitoring: matters of assessment, matters of validity. J. Pers. Soc. Psychol. 51, 125-139. doi:10.1037/ 0022-3514.51.1.125

Sobin, C., and Sackeim, H. A. (1997). Psychomotor symptoms of depression. Am. J. Psychiatry 154, 4-17. doi:10.1176/ajp.154.1.4

Speer, S. A. (2002). "Natural" and "contrived" data: a sustainable distinction? Discourse Stud. 4 (4), 511-525. doi:10.1177/14614456020040040601

Starcevic, V. (1995). Pathological worry in major depression: a preliminary report. Behav. Res. Ther. 33, 55-56. doi:10.1016/0005-7967(93)e0028-4

Steger, M. F., and Kashdan, T. B. (2009). Depression and everyday social activity, belonging, and well-being. J. Couns. Psychol. 56(2), 289-300. doi:10.1037/ a0015416

Stevanovic, M. (2013). Constructing a proposal as a thought: a way to manage problems in the initiation of joint decision-making in Finnish workplace interaction. Pragmatics 23(3), 519-544. doi:10.1075/prag.23.3.07ste

Stevanovic, M. (2015). Displays of uncertainty and proximal deontic claims: the case of proposal sequences. J. Pragmat. 78, 84-97. doi:10.1016/j.pragma.2014.12.002

Stevanovic, M., Himberg, T., Niinisalo, M., Kahri, M., Peräkylä, A., Sams, M., et al. (2017). Sequentiality, mutual visibility, and behavioral matching: body sway and pitch register during joint decision-making. Res. Lang. Soc. Interact. 50 (1), 33-53. doi:10.1080/08351813.2017.1262130

Stevanovic, M., Henttonen, P., Koskinen, E., Peräkylä, A., Nieminen vonWendt, T., Sihvola, E., et al. (2019). Physiological responses to affiliation during conversation: comparing neurotypical males and males with Asperger syndrome. PLoS ONE. 14 (9), e0222084. doi:10.1371/journal. pone. 0222084
Stevanovic, M., Tuhkanen, S., Järvensivu, M., Koskinen, E., Savander, E., Valkia, K., et al. (2021). Physiological responses to proposals during dyadic decisionmaking conversations. PLoS ONE. 16 (1), e0244929. doi:10.1371/journal.pone. 0244929

Stivers, T. (2015). Coding social interaction: a heretical approach in conversation analysis? Res. Lang. Soc. Interact. 48 (1), 1-19. doi:10.1080/08351813.2015.993837

Stivers, T., Mondada, L., and Steensig, J. (2011). The Morality of Knowledge in Conversation. Cambridge: Cambridge University Press.

Stivers, T., and Rossano, F. (2010). Mobilizing response, Res. Lang. Soc. Interact. 43 (1), 3-31. doi:10.1080/08351810903471258

Stivers, T. (2008). Stance, alignment, and affiliation during storytelling: when nodding is a token of affiliation. Res. Lang. Soc. Interact. 41 (1), 31-57. doi:10. 1080/08351810701691123

Tang, D., and Schmeichel, B. J. (2015). Look me in the eye: manipulated eye gaze affects dominance mindsets. J. Nonverbal Behav. 39 (2), 181-194. doi:10.1007/ s10919-015-0206-8

Tausczik, Y. R., and Pennebaker, J. W. (2010). The psychological meaning of words: LIWC and computerized text analysis methods. J. Lang. Soc. 29(1), 24-54. doi:10.1177/0261927X09351676

Thomson, J. J. (1976). Killing, letting die, and the trolley problem. Monist. 59 (2), 204-217. doi:10.5840/monist197659224

Thornborrow, J. (2015). The discourse of public participation media. New York: Routledge.

Vahey, R., and Becerra, R. (2015). Galvanic skin response in mood disorders: a critical review. Rev. Int. Psicol Ter Psicol. 15 (2), 275-304.

Vanheule, S., and Hauser, S. T. (2008). A narrative analysis of helplessness in depression. J. Am. Psychoanal. Assoc. 56(4), 1309-1330. doi:10.1177/ 0003065108325969

Voutilainen, L., Henttonen, P., Kahri, M., Ravaja, N., Sams, M., and Peräkylä, A. (2014). Affective stance, ambivalence and psychophysiology. J. Pragmat. 68, 1-24. doi:10.1016/j.pragma.2014.04.006

Wiklund, M. (2012). Gaze behavior of pre-adolescent children afflicted with Asperger Syndrome. Commun. Med. 9, 173-186. doi:10.1558/cam.v9i2.173

Williams, C., and Garland, A. (2002). A cognitive-behavioural therapy assessment model for use in everyday clinical practice. Adv. Psychiatr. Treat. 8, 172-179. doi:10.1192/apt.8.3.172

Zech, E., and Rimé, B. (2005). Is talking about an emotional experience helpful? Effects on emotional recovery and perceived benefits. Psychol. Psychother. 12(4), 270-287. doi:10.1002/cpp.460

Conflict of Interest: The authors declare that the research was conducted in the absence of any commercial or financial relationships that could be construed as a potential conflict of interest.

Copyright $\odot 2021$ Koskinen, Tuhkanen, Järvensivu, Savander, Valkeapää, Valkia, Weiste and Stevanovic. This is an open-access article distributed under the terms of the Creative Commons Attribution License (CC BY). The use, distribution or reproduction in other forums is permitted, provided the original author(s) and the copyright owner(s) are credited and that the original publication in this journal is cited, in accordance with accepted academic practice. No use, distribution or reproduction is permitted which does not comply with these terms. 


\section{OPEN ACCESS}

Edited by:

Antonio Bova,

Catholic University of the Sacred

Heart, Italy

Reviewed by:

Yuchen Shi,

East China Normal University, China Céline Miserez-Caperos, Haute École Pédagogique BEJUNE,

Switzerland

*Correspondence:

Kalypso Iordanou Klordanou@uclan.ac.uk

Specialty section: This article was submitted to

Language Sciences, a section of the journal Frontiers in Psychology

Received: 19 November 2020 Accepted: 26 January 2021

Published: 04 March 2021

Citation:

lordanou K and Rapanta C (2021) "Argue With Me": A Method

for Developing Argument Skills.

Front. Psychol. 12:631203.

doi: 10.3389/fpsyg.2021.631203

\section{"Argue With Me": A Method for Developing Argument Skills}

\author{
Kalypso lordanou ${ }^{1 *}$ and Chrysi Rapanta ${ }^{2}$ \\ 1 School of Sciences, University of Central Lancashire, Larnaka, Cyprus, ${ }^{2}$ Faculty of Social Sciences and Humanities, \\ Universidade NOVA de Lisboa, Lisbon, Portugal
}

Philosophers, psychologists, and educators all acknowledge the need to support individuals to develop argument skills. Less clear is how to do so. Here, we examine a particular program, the "Argue with Me" dialogue-based pedagogical approach, having this objective. Reviewing approximately 30 studies that have used the "Argue with Me" (AWM) method with students of different backgrounds and educational levels-primary, middle, high school, and university-across five different countries, we examine its strengths and limitations in terms of what develops and how this development occurs. Dense engagement in goal-based activities involving extended dialogic practice and reflection is shown to be effective in fostering argument skills and dispositions. Studies examining the mechanisms of such development identify the role of meta-level understanding regarding the purpose of argument. This understanding is epistemological in nature and supports the development of dialogic skills at the strategic level. In addition to examining the AWM method as a means for supporting the development of argument skills, this review examines how empirical research employing the method in varying contexts provides insights into the nature of argument skills and their development, as well as the relations between argument skills and other skills or forms of understanding. For instance, we examine how studies employing the AWM method answer questions such as "How general or content-specific are argument skills?" or "How do dialogic argument and individual written or spoken argument connect as they develop?" We address these questions by examining evidence regarding the transfer of gains across topics, domains, and individual vs. dialogic modes of expression. Finally, the pedagogical implications of the "Argue with Me" approach are discussed, especially with regard to its potential both as a standalone method for developing argument skills and integrated into traditional literacy and social studies curricula.

Keywords: argumentation, literacy, epistemology, critical thinking, writing, curriculum, citizenship

\section{INTRODUCTION}

The topic of argument skills is as old as the existence of human thought about reasoning, which came to light with some early philosophers' work in ancient Greece and Rome, with Aristotle and Cicero the most representative examples. Among other contributions, Aristotle distinguished between the different types of common places (topoi) for logical premises to be drawn on, while 
Cicero highlighted the indispensable connection between the logical construction of arguments (invenire) and their rhetorical elaboration (orare). The art of dialectics ( $\delta\llcorner\alpha \lambda \varepsilon \kappa \tau \iota \kappa \dot{)}$ ) was born and, along with it, the need for methods to ensure the construction of better (more logical and more persuasive) arguments. The need to gain an understanding of the links between logic, rhetoric, reasoning, and cognitive development has been a pressing one since ancient times.

In recent years, there is an increased interest in research on argumentation (Nussbaum, 2008; Garcia-Mila et al., 2013; Reznitskaya and Gregory, 2013; Murphy et al., 2018; Resnick et al., 2018; Larrain et al., 2020). In this article, we focus on the ideas and research data reflected in a particular line of research, the Argue with $\mathrm{Me}$ (AWM) approach, to developing argument skills and dispositions developed by Kuhn and colleagues (Kuhn et al., 2016a). The need for this theoretical and empirical overview emerges from a current lack of a qualitative synthesis explicitly focusing on studies that have implemented this innovative pedagogical method, on one hand, and an increasing evidence that this method works when it comes to argument skills' development, on the other. One aim was to add to the understanding of what develops and the mechanisms that support this development. In addition, we aimed to identify pedagogical implications as well as directions for current and future research into the still underexplored paths of argument skill development.

The article is structured as follows. Firstly, a synthesis of the major theoretical assumption behind Kuhn's dialogical argumentation pedagogical method will be presented. After this theoretical framing, we will pass to the empirical part of our review, making explicit its concrete questions that guided our analysis of approximately 30 studies implementing the AWM curriculum until today. Conclusions and recommendation emerging from this analysis will be presented at the end.

\section{THEORETICAL ASSUMPTIONS OF THE ARGUE WITH ME CURRICULUM}

Influenced by the twentieth-century psychologist Billig (1987) and the argumentation theorist Douglas Walton (1989), Kuhn makes the following series of claims.

\section{Everyday Thinking Is by Nature Argumentative}

Rooted in the early origins of informal reasoning, as a form of reasoning aiming at dealing with everyday problems and decisions, there lies the idea that the greatest part of human thinking is about ill-defined issues, and as such, a kind of thinking appropriate for resolving those is necessary. This thinking must focus on assessing, weighing, and using the information available as relevant and adequate to support one's position leaning toward an action or a belief. Many have named this thinking critical thinking, with argument construction and evaluation being one of its main goals. Kuhn, however, takes a step further: combining critical thinking and informal reasoning theories, she claims that a view of thinking as argumentation is necessary, one that goes beyond a thinking performance using valid arguments and sees argumentation as a main practice path toward the development of skills necessary for citizenship in a democratic society. The idea of argument as thinking (Kuhn, 1992) gained further insights later as a more comprehensive view of a dual relationship between argument as a critical thinking practice leading to more argumentative thinking products translated into better (more reasoned, sophisticated, and weighed) decisions and proposed solutions to everyday problems. Her most recent book titled Building our Best Future: Thinking Critically about Ourselves and Our World (Kuhn, 2018b), written directly to adolescents, explicitly focuses on the critical thinking practice of argumentative reasoning aiming at the informed decisionmaking and problem-solving practices of adolescents.

\section{The Argumentative Nature of Thinking Needs Dialogue}

Kuhn (1992) made the distinction between a rhetorical and a dialogic argument, the former referring to "a course of reasoning aimed at demonstrating the truth or falsehood of something" (American Heritage Dictionary, 1981, cited in Kuhn, 1992, p. 157) and the latter referring to an argument in course, meaning between at least two people. The same distinction is described by O'Keefe (1992) with the terms argument1 and argument2 types of argument and, later (Johnson, 2002), as argument-as-product (i.e., something that a person makes) and argument-as-process (i.e., something that a person engages in). Moreover, from an informal logic point of view, the argument structures (i.e., a set of propositions with certain characteristics) manifested within arguments-as-products, or rhetorical arguments, presuppose the process of argumentation within which they are produced (Johnson, 2002), i.e., the argument-as-process in which they emerge. In other words, rhetorical arguments are necessarily dialogic, in a twofold sense: (a) as part of their structure, as any reasoned argument at least implies an opposite or alternative viewpoint; otherwise, it would be a mere inference or reasoning, without an argument notion expressed within (for a distinction between argument and reasoning, see Walton, 1990), and (b) as part of their function, as an argument cannot be identified and/or assessed out of its context, and this context is necessarily communicative even when the argument is expressed intrapsychologically and not inter-psychologically, as for example in a speech/lecture or even in written discourse. These two assumptions have formed the basis of Kuhn's most recent thought, especially when it comes to the development of the idea that critical thinking is necessarily dialogic and manifested through the practice of argumentation (Kuhn, 2018a, 2019).

\section{Dialogue to Be Nurtured Needs Intentional Thinking}

If argument is necessarily dialogic, as explained above, then what characteristics does dialogue need to have in order to be productive? Although Kuhn and her colleagues do not use the term "productive" as a characteristic of educational dialogue as other scholars do (see for example Resnick et al., 2010), they imply that dialogue is productive when it leads to the 
development of argument skills, in oral or written discourse form. In other words, the productivity of dialogue is not a characteristic to be judged a priori or in the course of dialogue itself, for example when certain norms of dialogic behavior are met, as in the case of Mercer's (1995) exploratory talk, but only a posteriori, after the arguments produced are assessed as being reasoned and of a certain dialogic quality (i.e., dual, integrated, etc., in the case of written arguments; transactive, dialectical, etc., in the case of oral argument moves). However, what can and should be done a priori, in Kuhn's perspective, is the design of the argument learning environment on the basis of one main assumption: the goal-orientedness of the activities through which the gradual immersion in the argumentas-process nature and objectives is achieved. Although many educational researchers (e.g., Nussbaum et al., 2005; Nussbaum, 2008; Garcia-Mila et al., 2013) have suggested that the goal of argumentation made explicit to students must be one of collaboratively reaching a consensus, rather than convincing the other party (i.e., a "win-win" rather than a "win-lose" situation), Kuhn has opted for maintaining the dialectical nature of persuasive argumentation while at the same time framing it as a collaborative activity. In Kuhn's account, an argument is like a ball that needs to be successfully hit from one side to the other in order for the game (argument-as-process) to be on. At the end, the party that has made the best hits wins. This view of persuasive argumentation as a participation in a collaborative, yet competitive, game has several pedagogical implications: (a) that each party needs to have several instances of intra-team collaboration in order for their hits to be as successful as possible; (b) that each party needs to anticipate the other party's hits in order to receive them and respond to them adequately; and (c) that both parties are interested in the game going on, therefore receiving and replying to the other party's hits throughout the course of the game. From an argumentation theory point of view (Walton, 1989), the several "hits" aim at increasing the strength of the argument at hand, when it comes to one's own arguments, or decreasing it, when it comes to responding, implicitly or explicitly, to the other side. Therefore, the goal of argumentation is dual: on one hand, one must search to "secure commitments from the opponent that can be used to support one's own argument"; on the other hand, (s)he must "undermine the opponent's position by identifying and challenging weaknesses in his or her argument" (Kuhn and Udell, 2003, p. 1246).

The preceding ideas gave gradual rise to the development of a method aiming to support individuals' argument skills, called "Argue with Me" (AWM). The method, first fully implemented by Kuhn et al. (2008), with earlier versions by Felton (2004) and Udell (2007), involves extensive practice in argumentation and reflection in the context of a goal-based activity that keeps participants' motivation high. Since 2008, the method has been implemented in many schools, with consistent findings. Currently, there are 29 empirical papers describing studies where the AWM method has been implemented. The duration of the AWM intervention ranged from as short as six intervention hours over 2 days (Iordanou et al., 2019) to longitudinal twiceweekly implementations, up to 3 years (Crowell and Kuhn, 2014).
AWM is structured into three main phases-Pregame, Game, and Endgame-with different cognitive and dialogic objectives in each. Table 1 presents a summary of the activities and specific cognitive and dialogic goals at each phase, while Figure $\mathbf{1}$ depicts the sequence.

The goal of this paper was to provide an overview of the major outcomes of the 29 empirical studies that have applied the AWM method thus far.

\section{WHAT DEVELOPS?}

The development of argument skill is multifaceted and gradual (Kuhn et al., 2013). The work by Kuhn and colleagues implementing the AWM method in different contexts and with the manipulation of different variables sheds light on the complex nature of argument skill development and its underlying mechanisms. In this section, we seek to identify the particular gains in argumentive competence empirically related to the AWM implementation, as well as the specific characteristics of the dialogue-based method that may promote one gain or another. Table 3 shows the gains of engagement in the AWM curriculum in all the studies that the AWM method has been implemented.

Argumentive reasoning development encompasses two main manifestations: the first is related to the production of valid arguments, either individually or interactively, while the second is related to relevant forms of participation in argumentive dialogue. These two manifestations, previously described in the Introduction as argument-as-product and argument-as-process, will now be given a closer look in terms of their specific development reported in the AWM-related empirical research. We then proceed from skill development to gains in content knowledge.

\section{Constructing Valid Arguments}

A valid argument can be represented by an idea unit containing a claim supported by a piece of information supporting that claim. This idea of functional support is highly important as it represents informal logic criteria, such as sufficiency and acceptability, as described by Blair and Johnson (1987). For an idea to be sufficiently supported, the selected information must be linked to it clearly and explicitly enough for the logical relation between the two to be revealed. According to Toulmin (1958), this kind of logical relation can be of two types: (a) an explanation of how what is claimed to be a support naturally links to the claim itself (warrant in Toulminian terms) or (b) a justification of why this specific linkage between claim and support must be considered as evidence that the claim is true (backing in Toulminian terms). Without establishing connections neither with the informal logic criteria nor with Toulmin's theoretical contribution of a valid argument structure, Kuhn and colleagues seem to be claiming something very similar with their simplified notion of a functional unit: for a claim to be characterized as evidencebased, the connection between the alleged evidence and the 
TABLE 1 | Cognitive and dialogue objectives of the three phases of the "Argue with Me" (AWM) method and its main activities (adapted from Kuhn et al., $2016 a)$.

\begin{tabular}{|c|c|c|}
\hline Cognitive objectives & Dialogue objectives & Main activities \\
\hline \multicolumn{3}{|l|}{ Pregame } \\
\hline $\begin{array}{l}\text { Understand that reasons underlie opinions, different } \\
\text { reasons exist for the same opinion, and some reasons are } \\
\text { better than others. }\end{array}$ & $\begin{array}{l}\text { Elaborate argument blocks using } \\
\text { reasons (evidence) to support opinions. }\end{array}$ & $\begin{array}{l}\text { Small-group brainstorming, one-to-one ideas' } \\
\text { elaboration and synthesis, and small-group analytical } \\
\text { discussion }\end{array}$ \\
\hline \multicolumn{3}{|l|}{ Game } \\
\hline $\begin{array}{l}\text { Understand that opponents have reasons too, reasons can } \\
\text { be countered, and counters to reasons can be rebutted. }\end{array}$ & $\begin{array}{l}\text { Generate counterarguments to other's } \\
\text { reasons and rebuttals to one's } \\
\text { counterarguments. }\end{array}$ & $\begin{array}{l}\text { Dyadic written or semi-oral discourse, pair-to-pair } \\
\text { confrontation, and within-pair reflection }\end{array}$ \\
\hline \multicolumn{3}{|l|}{ Endgame } \\
\hline $\begin{array}{l}\text { Understand that the same information can be used as } \\
\text { evidence to support or weaken different claims. }\end{array}$ & $\begin{array}{l}\text { Weigh opposing positions in a } \\
\text { framework of alternatives and evidence. }\end{array}$ & $\begin{array}{l}\text { Small-group reflection, one-to-one debate, whole-class } \\
\text { reflection, and individual writing }\end{array}$ \\
\hline
\end{tabular}

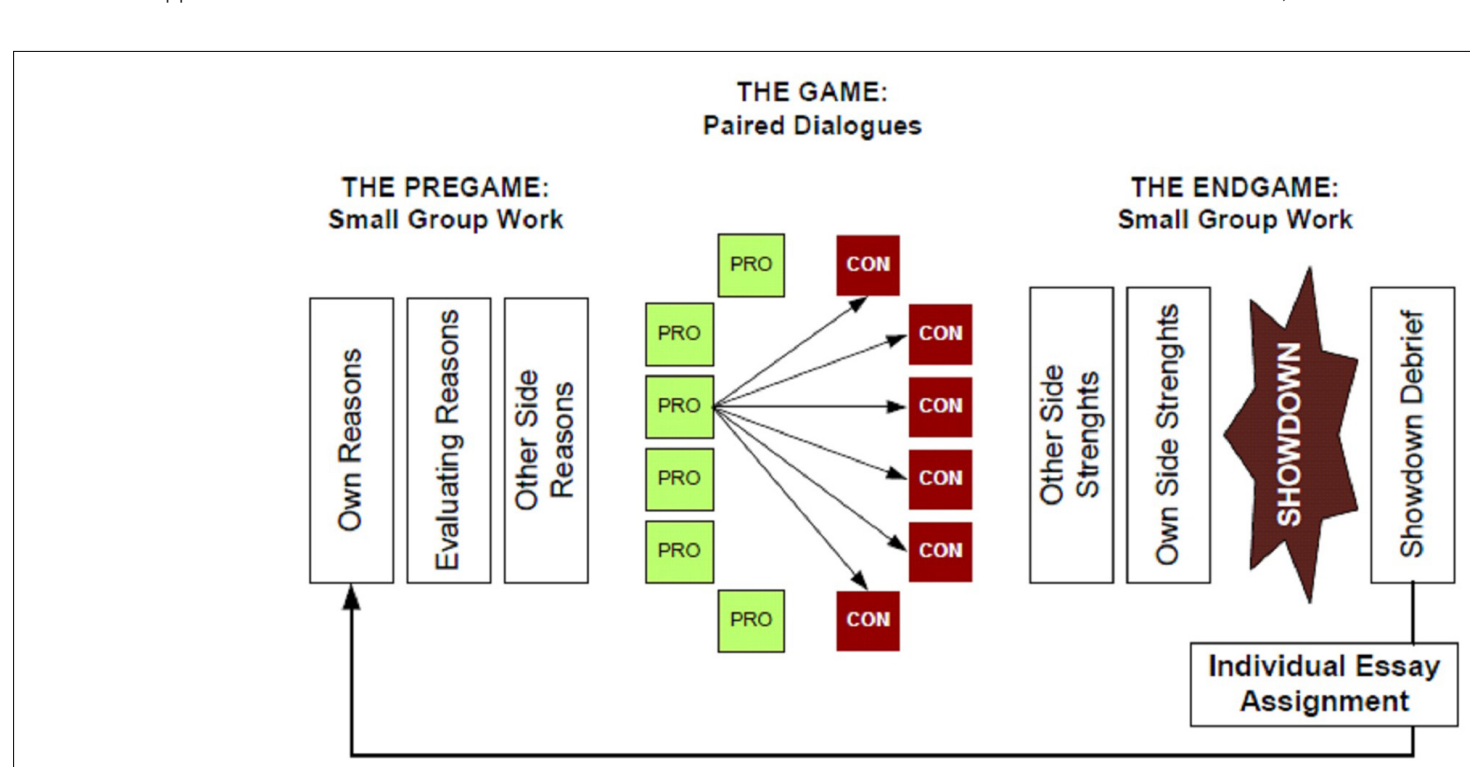

FIGURE 1 | The AWM structure.

claim must be explicit, and the evidence must be accurate (not "mischaracterized;" Shi et al., 2019, p. 118), meaning that the original meaning and context of the information serving as evidence must not be altered, as in the case of falsification of information in social media fake news and stories. Moreover, the use of factual information as evidence must be done in a way that is logically acceptable, meaning not violating the standards of soundness and cohesion (Blair and Johnson, 1987). This, in Kuhn's terms (Kuhn et al., 2013), corresponds to a commitment to "accountable talk" (Michaels et al., 2008), implying that the claims put forward, both proposing and supporting ideas, are based on shared standards of reasoning and knowledge (otherwise the use of information would be fallacious or paralogical; see Rapanta and Walton, 2016). All of the above aspects constitute what can be called evidencebased reasoning. What makes Kuhn's contribution unique, however, is her conceptualization of evidence not as a static entity with an a priori given status but as a functional unit itself, subset to pragmatic modifications according to its use. Information becomes evidence when employed in relation to a claim.
Pragmatic modifications can be of four main types, namely data used to support one's own view, data used to support the other party's view, data used to weaken the other side's view, and data used to weaken one's own view. These different functional uses of information as evidence give validity to an argument, as it is not the evidence itself that is more valid than another. This view explains also the fact that, for Kuhn (see Kuhn and Moore, 2015; Kuhn, 2015), two types of evidence (or better said information used as evidence) are possible: the shared, i.e., based on a set of information made available to students, and the personal, i.e., generated from the students' own personal knowledge. ${ }^{1}$ For both types, the same criteria of functionality apply. Table 2 illustrates the identification of functional units in an 11 year-old female Portuguese student essay, after she participated in the AWM curriculum, on the topic of whether we should immediately end the use of plastic or not.

\footnotetext{
${ }^{1}$ This openness to also consider personally known evidence in functional units' construction is highly important as it leaves space for pre-argumentation forms to emerge and develop throughout school-based practice. Examples of children's pre-argumentation strategies can be found in Bova and Arcidiacono (2014) and in Arcidiacono and Bova (2015).
} 
TABLE 2 | Rationale behind the identification of functional units (i.e., valid arguments) in a student's essay.

Text
"I think we should ban the plastic because it kills many animals, it pollutes the
environment, causes fires, and it causes the global warming."

"There are many alternatives to replace it, for example glass, metal, bambu?"

"Some time ago, I saw a documentary with my mum that the straws (among other plastics) because they are light they fly with the wind, they go to the sea, and the penguins (among other animals) were eating them thinking that they were food and it stayed in their bellies it was giving them the feeling that they were full and they didn't manage to eat."

"And the animals are food of other animals."
Functional (FU) and non-functional (NF) unit identification with explanation

This sequencing of reasons without a further connection to the claim would have been coded as NF if the student did not continue to explain each one subsequently. Therefore, we assume it is just an introduction to her reasoning that follows.

The existence of alternatives to the plastic was among the information provided to students in a Q\&A format. Therefore, we consider this as a shared FU.

Here, the student presents a piece of personal knowledge as evidence for her claim previously made in her short introduction (i.e., it kills many animals). Therefore, we consider this as a personal FU.

This further reasoning misses an important link (i.e., if some animals die, more animals would do so) to be considered as FU. Therefore, it is NF.
A necessary counterpart of evidence-based reasoning is the skill of antilogos, namely the ability to identify contrary commonplaces to one's own assumptions and positions, which may lead to totally different or even oppositional claims and positions (Billig, 1987). Coherent to the idea that evidence relates to one's own understanding of something that "if found and correctly understood, could change one's knowledge, one's beliefs, concerning some matter" (Buckland, 1991, p. 353), reasoning deriving from evidence may lead toward one conclusion or another based on its interpretation and use each time. This is why for Kuhn and colleagues, any construction of a valid argument implies coordination between claim and evidence (Kuhn and Moore, 2015; Hemberger et al., 2017). Such coordination, if successful, allows dialogue participants not only to argue against an opponent but also to adequately reply to his/her counterarguments by means of a rebuttal (Kuhn et al., 2008). Both counterarguments and rebuttals, and the different strategies used to express them in a dialogue, are manifestations of the antilogos skill, which in turn is an essential ingredient of critical thinking and argumentation (Walton, 1989).

Addressing the question of what aspects of evidencebased reasoning and antilogos skills are promoted as result of the AWM curriculum, a common finding across the empirical studies reviewed is that, following participation, students more often search for and use evidence in their efforts primarily to support their own and undermine the other's position, but also to a lesser extent to address evidence and arguments incongruent with their own position (Kuhn et al., 2008, 2016b; Iordanou and Constantinou, 2015; Kuhn and Moore, 2015; Hemberger et al., 2017; Shi, 2019; Shi et al., 2019; Iordanou and Kuhn, 2020), and more efficiently (Iordanou, 2010, 2013; Crowell and Kuhn, 2014; Kuhn and Crowell, 2011; Iordanou and Constantinou, 2014; Papathomas and Kuhn, 2017; Matos, 2021). This behavioral, as contrasted to the epistemological (discussed below), increased facility with what counts as evidence and how it can serve one's argumentive reasoning is a central benefit of the AWM curriculum.

But what are the particular aspects of the dialoguebased method that render these gains possible? The AWM method is a complex, multicomponent intervention, and specific experimental dissection is required to isolate its effective components. Some of the reviewed studies suggest the dyadic intense dialogic interaction taking place during the Game, and characteristics of it thereof, as a major factor leading to argument gains, whereas others focus on particular elements of the AWM method to address this question, examining for example the role of reflective activities or the type and order of relevant information made available to the students during Pregame and Game. Each of these components is examined below.

\section{Dyadic Intense Dialogic Interaction}

The idea that dyadic argumentation is a means of cognitive engagement is rooted in the Vygotskian tradition highlighting the complementarity of social and internal thinking. A pioneering study by Kuhn et al. (1997) not only supports this view but also highlights the types of cognitive gains the dialogical argumentive engagement with a peer may lead to. This study, which was a predecessor of the Game phase of the AWM method, showed that, after their systematic immersion in dyadic argumentation over 5 weeks, both adolescents and adults showed evidence of reasoning improvement. Gains included shift from one-sided to two-sided arguments, arguments based within an alternatives framework, and metacognitive awareness of the coexistence of multiple views. The study additionally suggested how different forms of dialogic interaction contributed to different forms of change.

Subsequent studies implementing the AWM method further confirmed the role of intensive dialogic engagement in argument skill development. For example, Iordanou and Constantinou (2015) compared students who engaged in the AWM method in the context of a web-based learning environment, SOCRATES, which included a rich database on the topic of climate change. Eleventh graders serving in the experimental condition engaged in the AWM method, while a group of peers studied the same database for the same amount of time but did not engage in an argumentive discourse activity. Students in the experimental condition increased use of evidence in their dialogues, used more evidence that functioned to weaken the opponents' claims, and used evidence more accurately. Iordanou 
and Kuhn (2020) examined which forms of dyadic interaction are more beneficial, comparing individuals who engage in discourse with peers who hold an opposing view with individuals who engaged in discourse with peers who hold the same positions as themselves. Young adolescents were given access to identical relevant evidence and engaged in dialogues on a physical science topic. In the experimental condition, electronic dialogues were conducted with a series of peers who held an opposing view; in the control condition, dialogues were confined to same-side peers. The results showed differences in the extent and types of functional evidence-based argumentive idea units in individual final essays on both the intervention and a transfer topic, favoring the experimental condition. Extension of the study longitudinally to a second year with a new topic showed continued gains and condition differences, with the experimental group surpassing the control group. This study further suggests that adversarial argumentation, employing the aim to persuade, is a more productive means, compared to coalescent or collaborative argumentation, to support the development of argument skills. Matos (2021) showed that incorporating a collaborative writing activity with opposing side pairs in the AWM method yielded greater gains in terms of using evidence and integrating belief-incongruent and beliefcongruent statements compared to engagement in the AWM method without this additional element.

Another group of studies aimed at examining whether the skills of the partner that one collaborates with while engaging in the AWM method function as a further scaffold for the observed individual argument gains. Zillmer and Kuhn (2018) found that peer collaboration, that is, having same-side peers to collaborate while they engage in discourse with other pairs holding opposing position, supported individuals' argumentation skills. The benefits of collaboration extended to equal-as well as unequal-ability peers, a condition not emphasized in Vygotsky's writing. Same-ability partners could flexibly scaffold one another with metacognitive support, taking on the role of support provider and support recipient interchangeably and as needed.

\section{Reflective Activities}

Another core element of the AWM method is engagement in reflective activities about one's own argumentation. Significant advancements in students' use of evidence were observed after their engagement in reflective activities that prompted them to reflect on the use of evidence in their arguments, when students' progress was examined using a microgenetic method (see Iordanou and Constantinou, 2015). When an additional reflective activity about evidence was included in the AWM method, it proved more effective in promoting students' use of both types of evidence (congruent and incongruent) and therefore a superior argument performance compared to the AWM method without this additional reflective activity or to a regular school curriculum (Shi, 2019). This was particularly evident in the construction of "However" arguments, i.e., pairs of two units with one unit supporting the opposing side or weakening one's own side immediately accompanied by a unit supporting one's own or weakening the other side. In their essays on the last intervention topic (topic 3) in Shi's study, the majority of students successfully coordinated claims and (incongruent) evidence at least once. Iordanou (submitted) directly investigated the role of reflection in the AWM method by comparing a group who engaged in the AWM curriculum with another one that engaged in the AWM method but not in reflective activities. The results similarly showed that the condition that engaged in both reflective and dialogic activities outperformed the condition that engaged only in dialogic activities.

\section{Use of Scaffold Prompts}

Some of the reviewed studies used prompts for reflection or to encourage the use of evidence. Incorporating scaffold prompts that exemplified functions of evidence in relation to a claim accelerated the prevalence of evidence-based claims in essays of low-performing middle schoolers compared to participants in the same year-long dialogue-based intervention who received no or a limited form of evidence prompts (Hemberger et al., 2017) or compared to participants who engaged in their regular school curriculum (Shi et al., 2019). Iordanou et al. (2019) gave particular attention to the specific types of prompts accompanying the use of questions and answers (Q\&As) provided to students. In one experimental condition, the standard prompt "Try to use this information in your arguments" was used, whereas for the second experimental condition, the subtracted prompt "Here's some information about the topic" was used. They found no significant difference between these conditions; rather, the gradual presentation of evidence both congruent and incongruent to one's own position was the condition that had some significant impact on students' performance. This mixed evidence presentation was further accompanied by a prompt of the type "Not all of the evidence is going to support your side; if it doesn't, see if you can deal with it." This prompt to consider incongruent evidence showed the greatest effect in furthering mastery of a critical argument skill-to acknowledge and address, rather than ignore, evidence that counters one's favored position.

\section{Type and Order of Relevant Information (Evidence) Made Available to Students}

Hemberger et al. (2017) tested the hypothesis of whether the type of information presented to students in a Q\&A format had an impact on the type and quality of arguments produced. The results showed that in topic 1 , the students who received pieces of evidence supporting "own side" only did better than the students who received multiple kinds of evidence-supporting own position, weakening other position, supporting other position, and weakening own position-in terms of producing functional (evidence-based) statements, reflecting the easier task they had of using only supporting evidence. However, in the subsequent topics, the latter group, who received multiple kinds of evidence, surpassed the first one, who received only "own side" evidence, in the frequency of evidence-based statements despite their more challenging task of using multiple kinds of evidence. Students who were not given any evidence showed relatively little evidence use, of the self-generated (from the individual's personal knowledge) type, only in the later topics. 
Iordanou et al. (2019) compared the traditional information text format of presenting relevant knowledge to students with the intentionally structured Q\&As gradually provided during the AWM Pregame and Game phases. Although students in both groups became successful in making functional use of the evidence available to them, greater improvement was observed in the Q\&A condition. This improvement was significant also in the use of the "weaken other" type of evidence, which is noteworthy given the fact that the use of evidence to weaken a claim is more challenging than the use of evidence to support a claim (Hemberger et al., 2017). A subsequent study (also reported in Iordanou et al., 2019) focused on the order of the presented Q\&As, i.e., whether the facilitative order previously presented (Hemberger et al., 2017) made a difference. They found that although the order did not play a significant role, the gradual presentation of evidence that is both congruent and incongruent with one's own position did indeed result in greater student gains, as further explained in the next section.

A subsequent study by Shi (2019) focused on the prompted mixed evidence condition. Instead of presenting the congruent and incongruent evidence all at once, Shi (2019) opted for sharing one Q\&A piece of evidence at a time, for each Game dialogue session. In addition, students were also encouraged to formulate questions they wished to have answered, which the research team provided at a subsequent session. This addition increased the use of claim-congruent evidence ("support own" and "weaken other").

\section{Participating in Argumentive Discourse}

Relevance as a characteristic of discourse participation implies more than that contributions are on the topic and coherent. In skilled argumentive dialogue, an important role is played by structural relevance, i.e., how the argument components logically interrelate (Macagno, 2016). Another aspect of relevance refers to consistency with pragmatic function, i.e., the purpose of the dialogue and the different forms it may take throughout interaction (Macagno, 2016, 2019). In skill development terms, the structural form of relevant participation is captured in strategic and metacognitive skills, whereas pragmatic relevance is expressed in terms of metastrategic and epistemological awareness. Both are of core importance within the AWM curriculum and are manifested in particular gains, as elaborated below.

A recent review by Iordanou et al. (2016) shows how argumentive reasoning, including both the construction and evaluation of arguments, and epistemic cognition, i.e., "an umbrella term encompassing all kinds of explicit or tacit cognitions related to epistemic or epistemological matters" (Chinn et al., 2011, p. 141), are intertwined. In this section, we show how this interrelation is manifested and achieved within the AWM curriculum, and in particular through components that promote students' metacognitive and epistemological development.

According to Moshman (2015), epistemic cognition can be both domain-specific and domain-general, and the same applies for epistemic development, which is the progress in epistemic cognition. Similarly, for Kuhn (1999), there are three types of meta-knowing, namely the metacognitive, the metastrategic, and the epistemological knowing. The differences between the three are subtle but important to consider, especially when it comes to distinguishing the metacognitive from the other two types. For Kuhn (1999, 2001) and others (e.g., Swanson, 1990; Schraw, 1998), metacognition has a declarative component, namely a verbal aspect that directly contributes to one's (self-)regulation of knowing. Therefore, when we talk about metacognitive skills, we assume that there is an explicitly verbal behavior that shows that an individual is aware of his/her own knowledge. Manifestations of such verbal behavior occur when participants label the argument components (e.g., "here are my counterarguments against your position," "that's my evidence against yours," etc.) or when they talk about their own understanding of the reasons and evidence to support those (e.g., "this evidence supports that reason," "I don't know how to use this information to counter the other party," etc.). However, very often, these explicit verbal expressions do not take place, and self-regulation of knowledge is implicit and occurs together with other verbal behaviors that imply such self-regulation. This is so in the case of strategic use of evidence or of language to defend one's own position. Examples of these strategic metacognitive skills include the use of discursive strategies to defend one's own or counter another's position, which can vary from a simple clarification of one's own premises to more advanced counterargument and rebuttal strategies (e.g., undermining).

Empirical research applying the AWM curriculum has shown that students' immersion in a sequence of dialogic activities with a different setting and goal has been proven effective in the use and development of both oral and written strategic argumentive discourse. ${ }^{2}$ For example, the structured engagement in pair-to-pair dialogue, often mediated by electronic means, has been shown effective in immediate (e.g., Mayweg-Paus et al., 2015; Papathomas and Kuhn, 2017) and gradual (Kuhn et al., 2008, 2013; Kuhn and Crowell, 2011; Crowell and Kuhn, 2014; Iordanou et al., 2019; Iordanou, submitted) advancement in students' counterargument and rebuttal strategies.

When it comes to writing, extensive research (Crowell and Kuhn, 2014; Kuhn et al., 2016a; Hemberger et al., 2017; Iordanou et al., 2019; Iordanou, in press; Shi et al., 2019) involving implementation of the AWM curriculum has shown that dense engagement in oral argumentive interaction benefits the construction of two-sided texts. Such two-sidedness is also reflected in the formation of either a dual or the more advanced integrated perspective. Texts adopting a dual perspective recognize at least once the existence of a contrary or alternative perspective to one's own, whereas integrated argumentive texts include at least one sequence of adjacent statements of opposing perspectives. Emergence of this "However" argumentive structure (Kuhn et al., 2016b) has been a frequent marker of argumentive reasoning gains.

\footnotetext{
${ }^{2}$ In Kuhn's approach, argumentative writing is highly dialogic. This is why we opt for including it in the section titled "Participating in Argumentative Discourse." Similar to academic writing, writing an argumentative essay is like entering a room in which discussion on a debatable topic has already started (Graff and Birkenstein, 2010), and therefore, it is the writer's responsibility to be relevant to this previous and ongoing discussion.
} 
Another group of epistemic skills, more representative of the "meta" aspects of reasoning, are the so-called metastrategic skills. These refer to: (a) meta-level awareness of the goals of argumentive discourse and the strategies used to achieve them (Kuhn and Udell, 2003; Iordanou and Constantinou, 2014), also called metastrategic awareness (Shi et al., 2019), and (b) epistemological understanding of what constitute acceptable claims, what are acceptable forms to advance those in discourse, and what are the dialogue norms that need to be respected for this to take place (Kuhn et al., 2013; Kuhn and Zillmer, 2015). Empirical research implementing the AWM curriculum has shown that participants develop their epistemic understandings regarding argumentation (Kuhn et al., 2008, 2013; Iordanou, submitted) during their participation, but also their more general epistemological understanding of what is knowledge and how one knows (Iordanou, 2010, 2016; Kuhn et al., 2013; Zavala and Kuhn, 2017; Shi, 2020b).

\section{Acquiring Knowledge}

The "learning to argue" and "arguing to learn" distinction (Von Aufschnaiter et al., 2008; Muller-Mirza and Perret-Clermont, 2009) implies that any intervention primarily aiming to develop argument reasoning gains belongs to the former, while any study primarily seeking knowledge acquisition gains belongs to the latter. The AWM method is representative of a method designed with argument reasoning gains as a primary objective, but content knowledge gains have also been significantly observed, showing that learning-to-argue and arguing-to-learn objectives can be the result of engagement in a single curriculum (Iordanou et al., 2019).

For example, Rapanta (submitted) examined the implementation of the AWM method across four different subject areas by middle-grade teachers in Portugal after a $12 \mathrm{~h}$ professional development training focusing on the structure, goals, and activities of the method. As the method was adapted and integrated as part of the curriculum in history, language, citizenship education, and science, the goal of students also achieving content-related gains was explicit. In total, 145 adolescents ranging from the seventh to the 10th grades significantly improved their written answers to an open-ended test question in their corresponding subject areas. This question, chosen by the teachers, was used twice as a pre/post-test assessment and was unrelated to the topics of the intervention. This result, further supported in teacher interviews, shows that argument knowledge construction goes hand in hand with content knowledge construction, as reasoning and knowledge are highly interconnected with one "serving" the other (Iordanou et al., 2016).

In another study, Iordanou et al. (2019) examined the effectiveness of the AWM curriculum in fostering middle-school students' knowledge acquisition as well as dialogic and written argumentation skills with respect to a content-rich, socially significant topic. The results of two studies, one involving a physical science topic (study I; Iordanou et al., 2019) and the other a social topic (study II; Iordanou et al., 2019), showed that a single intervention could meet both objectives-"learning to argue" and "arguing to learn." The findings of Iordanou et al. (2019) showing argumentation skill and knowledge gains in the context of a single curriculum have been replicated later by other researchers (Larrain et al., 2020).

\section{MECHANISMS OF ARGUMENT SKILL DEVELOPMENT}

The strength and value of instructional approaches lie in their ability to promote knowledge or skill gains beyond the specific context of instruction, given that transfer of learning is considered the ultimate objective of teaching (McKeough et al., 2013). Participants who engage in the AWM curriculum exhibit evidence of transfer beyond the intervention context in which development occurred (see Table 3).

Following engagement in dialogic argumentation in the context of the AWM curriculum, individuals' transition from egocentric presentation of their own perspective to addressing the other side's perspective and using counterarguments was apparent not only in peer discussion but also in students' individual writing (Kuhn et al., 2008; Iordanou and Constantinou, 2014; Hemberger et al., 2017; Iordanou et al., 2019; Shi, 2019; Shi et al., 2019; Iordanou and Kuhn, 2020) as well as in solitary dialogues (Shi, 2020a) the participants constructed. The gains developed during practice on the social plane transferred to the individual plane, consistent with Vygotsky's sociocultural framework of the interiorization of action from the social plan to the internal, individual, plane (Vygotsky, 1978).

Transfer of strategic gains was also observed to a novel, nonintervention topic. For example, gains in using counterarguments transferred from one physical science topic, e.g., alternative sources for producing electricity, to another physical science topic, e.g., genetically modified food (Iordanou and Kuhn, 2020), or from a social science topic, e.g., homeschooling, to another social topic, e.g., capital punishment (Kuhn et al., 2008). Transfer was also observed across knowledge domains, that is, across topics from different knowledge domains. A study by Iordanou (2010) comparing the AWM method in a physical science domain and in a social domain showed that transfer was evident in both directions; that is, students whose intervention focused on a social topic showed transfer to a post-intervention assessment of dialogic skill in a science topic and vice versa. However, a difference in the magnitude of transfer was observed, with only students in the science intervention condition able to transfer their skill gain to the social topic to the degree that these skills were mastered in the science topic. Thus, argumentive competence in the science domain is amenable to the same development as in the social domain, although specific engagement and practice within the science domain is warranted. The evidence for transfer across topics and generality observed is considerable, but we should not draw the conclusion that content makes no difference (Kuhn et al., 2013).

Furthermore, transfer was observed across communication modes. Many studies showed transfer from arguing with another person online via computer to writing, usually involving handwritten individual essays (Kuhn et al., 2013, 2019; Kuhn and Moore, 2015; Hemberger et al., 2017; Shi et al., 2019; Iordanou and Kuhn, 2020; Shi, 2020a). A study by 
Iordanou (2013) showed transfer from arguing on the computer to arguing face-to-face. Primary school students engaged in the AWM method via an instant messaging software on the computer. Although the participants initially exhibited limited ability in arguing both face to face and on the computer, by the end of the intervention, they exhibited significant advances

TABLE 3 | Gains of engagement in the "Argue with Me" (AWM) curriculum and evidence of transfer.

\begin{tabular}{|c|c|}
\hline Study & Intervention gains \\
\hline Kuhn et al. (2008): US sample & $\begin{array}{l}\text { Antilogos (counterargument and rebuttal) Quantity and } \\
\text { quality of reasons (genuine justifications) Increasing } \\
\text { meta-level usage (meta-directive) during the course of the } \\
\text { intervention }\end{array}$ \\
\hline Iordanou (2010): Cyprus sample & $\begin{array}{l}\text { Number and length of utterances and rebuttal strings } \\
\text { Antilogos (counterarguments, counter-critiques) }\end{array}$ \\
\hline Kuhn and Crowell (2011): US sample & $\begin{array}{l}\text { Antilogos (two-sided essays) Epistemological gains: greate } \\
\text { awareness of the relevance of evidence to argument }\end{array}$ \\
\hline Iordanou (2013): Cyprus sample & Antilogos (counterarguments and rebuttals) \\
\hline Kuhn et al. (2013): US sample & $\begin{array}{l}\text { Epistemological gain: metatalk becomes more reciprocal, } \\
\text { sustained with time, and focused on the argumentation } \\
\text { process Antilogos (counterarguments) }\end{array}$ \\
\hline Crowell and Kuhn (2014): US sample & Antilogos (counterarguments) \\
\hline
\end{tabular}

Iordanou and Constantinou (2014): Cyprus sample

lordanou and Constantinou (2015): Cyprus sample

Kuhn and Moore (2015): US sample Kuhn and Zilmer (2015): US sample

Iordanou (2016): Cyprus sample

Kuhn et al. (2016b): US sample

Mayweg-Paus et al., 2015: US sample Hemberger et al. (2017): US sample

Papathomas and Kuhn (2017): US sample

Zillmer and Kuhn (2018): US sample

Iordanou et al. (2019): Cyprus and US samples

Kuhn et al. (2019): US sample

Rapanta and Trovão (2019):

Portuguese sample

Shi (2019): Chinese sample

Shi et al. (2019): US sample

Iordanou and Kuhn (2020): Cyprus sample

Shi (2020b): Chinese sample

Shi (2020a): Chinese sample
Increase in evidence use (functional units to weaken the opponent's claims) Increase in meta-level talk about evidence

Greater use of evidence to weaken opponents' claims More accurate evidence and meta-level communication about evidence

More evidence-based claims New evidence integration

Gradual increase of metatalk statements and their acknowledgment by the opposing pair

Epistemological understanding

Number of functional idea units increase Antilogos (counterarguments)

Antilogos (counter-critique, counter-alternative, underminer) Evidence use

Antilogos (counterarguments)

Metatalk as a result of metacognitive regulation

Content knowledge acquisition Acknowledging and addressing incongruent evidence

Antilogos (counterarguments)

Increased use of functional units (with reasoning fallacies related to social representations still present) Increased use of "However" compound units

Use of evidence: greater use in a condition engaged in reflective activities devoted to evidence, in addition to the AWM reflective activities

Greater use of support own and weaken other evidence on intervention topic

Gains in evidence used to weaken other, weaken own, and support other

Metatalk grows more frequent, becoming increasingly focused on evaluating the source of evidence and better sustained over successive turns Gains in epistemological understanding (Livia task) and intellectual disposition to engage in argument and recognize its value

Counterargument and evidence use to justify claims

\section{Transfer of gains}

Transfer from social topics to a novel social topic and transfer from online dialogue to individual essays

Transfer across content domains: from social to physical science and vice versa

Transfer from online dialogue to two individual essays on new social topics (two-sided essays)

Transfer from electronic dialogue to face-to-face dialogue

Transfer of the two types of gains to dialogue evaluation and dialogue construction tasks

Transfer from a social intervention topic to new social topics and transfer from dialogue to an evaluation task of a dialogic argumentation sequence

Transfer from a physical science topic to a novel physical science topic

Transfer from a physical science topic to a novel socioscientific topic

Transfer from dialogue to individual essays

Transfer across social and science topics

Transfer across social topics and from dialogue to individual essays

Transfer from dialogue to individual essays on the intervention topic and a novel topic

Transfer from dialogues with a more capable other to peer-only dialogues on a new topic

Transfer from dialogue to individual essays on intervention topic

Transfer from dialogue to individual essays within citizenship education curriculum

Transfer from dialogue to written essays within citizenship education curriculum, two grades (seventh and 10th)

Transfer from dialogue to written essays on intervention and transfer topics

Transfer from dialogue to individual essays

Transfer from first to second year, with a new topic in the physical science domain

Transfer from dialogue to written constructed dialogue 
TABLE 3 | Continued

\begin{tabular}{|c|c|c|}
\hline Study & Intervention gains & Transfer of gains \\
\hline Iordanou (in press): Cyprus sample & $\begin{array}{l}\text { Greater diversity of arguments, taking multiple } \\
\text { considerations (both social and science-related) into } \\
\text { account Two-sided arguments }\end{array}$ & $\begin{array}{l}\text { Transfer from dialogue to individual essay on intervention } \\
\text { topic }\end{array}$ \\
\hline Iordanou (submitted): Cyprus sample & $\begin{array}{l}\text { More evidence used to weaken other's position } \\
\text { Improvement in metastrategic and epistemological } \\
\text { awareness }\end{array}$ & \\
\hline $\begin{array}{l}\text { lordanou and Fotiou (submitted): } \\
\text { Cyprus sample }\end{array}$ & Multiple-text comprehension Use of weaken-other evidence & Transfer from dialogue to individual essays \\
\hline Matos (2021): Brazilian sample & $\begin{array}{l}\text { More frequent evidence-based arguments and integration } \\
\text { of belief-incongruent statements with belief-congruent ones }\end{array}$ & Transfer from dialogue to essay on a novel topic \\
\hline $\begin{array}{l}\text { Rapanta (submitted): Portuguese } \\
\text { sample }\end{array}$ & $\begin{array}{l}\text { Increased use of functional units and "However" compound } \\
\text { units }\end{array}$ & $\begin{array}{l}\text { Transfer from "learning to argue" to "arguing to learn": } \\
\text { improvement in content-related reasoning tasks without } \\
\text { other type of training Gains observed across four different } \\
\text { disciplinary areas (natural science, history, language, and } \\
\text { citizenship education) }\end{array}$ \\
\hline
\end{tabular}

in both modes. The gains of practice in the electronic modeincreased levels of counterargument and rebuttal-successfully transferred to the face-to-face mode.

Transfer of AWM gains was also observed in other tasks. Iordanou and Fotiou (submitted) examined the effectiveness of engagement in dialogic argumentation in relation to its ability to promote multiple-text comprehension. The multipletext comprehension of primary school students who engaged in the AWM method was compared with that of a control group, who engaged in business-as-usual school curriculum. Only the experimental group improved in multiple-text comprehension. They showed progress in both argument skill and multipletext integration skill throughout engagement in the intervention. Engagement in dialogic argumentation can thus serve as a promising pathway toward multiple-text comprehension. Dialogic argumentation, in which a contrasting perspective is embodied in a "real" person, as in the AWM method, may have benefited thinking about the issue, probably by emphasizing that there indeed exists a flesh-and-blood other who supports such views (Mill, 1859/1996; Iordanou and Kuhn, 2020). Recognizing that alternative positions exist on an issue, an epistemological achievement, is fundamental for multiple-text comprehension (Britt and Rouet, 2012; List and Alexander, 2019). The integration of contrasting views requires one to appreciate the need for and recognize the value of engaging in this task in order to expend the effort to engage in it, a recognition only mature epistemological understanding can provide (Kuhn, 2020).

\section{Explaining Transfer of Gains}

The transfer of gains observed from the intervention context to novel ones shows that something is developing that, once developed, is then transferable to a context different from the one in which it has originally developed. What is developing that supports the transfer of gains in argument skills? What is the mechanism of transfer? Studies using the microgenetic method suggest that a metastrategic understanding of the norms of argumentation is developing and supports the development of argument skills (Kuhn et al., 2008, 2013; Iordanou and Constantinou, 2015; Kuhn and Zillmer, 2015; Shi, 2020b; Iordanou, submitted).
Kuhn et al. (2008) examined young adolescents' development of better meta-level understanding about argumentive discourse and its goals. They particularly examined the claim that developing a meta-level understanding of the goals of argumentation, namely engaging in one another's claims and undertaking to weaken them, as well as seeking acceptance of one's own claims, what Walton (1989) identifies as the dual goal of argumentation, is likely to support progress in the procedural aspect. In an attempt to heighten such awareness, Kuhn et al. (2008) have implemented three techniques. Firstly, they ask participants who shared the same position to work in pairs in engaging in dialogues with opposing pairs, promoting planning and evaluation within the same-side pair. Secondly, they ask participants to engage in an explicit reflective activity in which they contemplate a transcript of their own previous dialogues, made possible by the record preserved by the electronic medium. Using a microgenetic method, they examined the processes of change during a year-long intervention program involving the AWM method. Observation captured both argumentive strategies within the discourse, but also metatalk, defined as talk about the discourse as opposed to talk about the topic. Participants showed significant gains in meta-level talk over the course of the intervention, in addition to gains in the use of counterargument strategies aiming to weaken others' positions. These findings suggest that metal-level awareness and understanding of argumentation is developing through engagement in dialogic argumentation and supports development at the performance level.

Kuhn et al. (2013), further examining individuals' meta-level understanding while engaging in the AWM method over the course of 3 years, confirmed and extended these earlier findings. Increasing metatalk revealed students becoming more explicit in their understanding of argumentation norms over time. This metatalk became more reciprocal and sometimes took a directive character, with one member of the pair providing meta-level scaffolding for the other. Shi (2020b) recently provided further support of the findings of developing meta-level understanding, reporting also an increasing prevalence of metatalk in evaluating sources of evidence. 
Iordanou and Constantinou (2015); Shi (2019), and Iordanou (submitted) revealed the unique contribution of reflective activities within the AWM method in promoting individuals' meta-level understanding of argumentation. Iordanou and Constantinou (2015) asked students to engage in reflective activities, which, in addition to prompting students to reflect on whether they constructed counterarguments and rebuttals, as was the case in the Kuhn et al. (2008) study, prompted them to reflect also on whether they had used evidence in their counterarguments or rebuttals. The results showed significant gains in primary school students' meta-level talk as well as greater use of evidence-based counterarguments immediately after students engaged in reflective activities within the AWM method. Shi (2019) examined a group who engaged in additional reflective activities focusing on evidence, in addition to engaging in the reflective activities already a part of the AWM method that focus on the use of counterarguments and rebuttals. Compared with a group who engaged only in the reflective activities of the AWM method, Shi found that the additional reflection led to additional gains in argumentive writing, particularly in coordinating evidence and claim. Iordanou (submitted) employed an experimental design comparing two conditions: the AWM method vs. the AWM method minus its reflective activities (control condition). Participants who engaged in reflective activities related to argumentation, in addition to practice in argumentation-the AWM method-outperformed participants who only engaged in argumentation practice. The AWM group showed greater gains in argument skill-particularly in employing evidence to weaken an opposing position. Microgenetic analysis of dialogues during the interventions revealed a different pattern of progress across the two conditions. Experimental condition participants exhibited gradual and ultimately greater improvements at both the strategic and meta levels compared to the control condition participants.

This line of work shows that meta-level awareness and understanding of argumentation develop with extended practice in and reflection on argumentive discourse. Gains in meta-level awareness appear hand in hand with gains at the strategic level, suggesting a bootstrapping relation between performance at the strategic level and meta-level awareness and understanding of argumentation (Kuhn and Pearsall, 1998). The meta-level awareness and understanding that are developing involve both metastrategic knowledge, which is an understanding about how to produce, for example, a counterargument or use a piece of evidence, and epistemological understanding. The latter entails appreciation of counterargument and evidence as critical components of argumentation as well as recognition of the point of argument and value argumentation in influencing others (Kuhn and Crowell, 2011; Kuhn et al., 2013). Shi (2020b) examined Chinese middle school students' disposition to engage in argumentive discourse after participating in a program employing the AWM method. She found that participants showed greater endorsement of argumentive discussion as a valuable activity than did peers in a non-participating control group.

Another prominent epistemological achievement observed to develop among adolescents engaged in the AWM method is the appreciation of the subjective nature of human knowledge and acknowledgment of alternative interpretations. Growing attention to others' positions implies an implicit developing understanding that these are worthy of consideration. More direct evidence comes from an experimental study (Iordanou and Kuhn, 2020) comparing the collaborative (same-side) and adversarial (mixed-position) discourse conditions. The adversarial condition prompted more attention to evidence pertinent to the opposing position and greater gains in the use of evidence weakening the opposing position. Finally, examination of meta-level communication provides evidence of growing epistemological understanding reflected, for example, in requests to clarify whether an opponent's claim represents a personal view or is based on evidence (Kuhn et al., 2013; Shi, 2020b). Even more direct evidence, based on explicit measures of epistemological understanding, is also available, confirming advances in epistemological understanding following engagement and practice in dialogic argumentation (Iordanou, 2010, 2016; Zavala and Kuhn, 2017; Shi, 2020b).

\section{PEDAGOGICAL IMPLICATIONS AND OPEN PATHS FOR FUTURE RESEARCH AND APPLICATION}

The review of the literature on the dialogue-based curriculum proposed by Kuhn and her colleagues indicates that it constitutes a powerful approach to fostering dialogic skills and for extending those skills to new content and to students' individual writing. The remarkable consistency with which the findings of the AWM curriculum have been replicated in almost 30 studies, in different countries worldwide-the United States, Europe, and Asiasince its early implementations shows that it has broad potential either as a stand-alone curriculum for developing argument skills or contextualized within different knowledge domainssocial, physical science, socioscientific-and the gains have the potential to transfer to novel topics, domains, communication modes, and tasks.

According to Wilson and Bai (2010), for students to become metacognitive and therefore self-regulated learners, teachers should engage students in problem-solving activities, allowing them to share their thinking and discuss their problem solving, to generate their own questions, and to explain their answers. All of these activities are present in the AWM curriculum. In addition, the AWM method goes a step further: not only do students learn how to work independently and collaboratively, as may happen in many structured inquiry-based learning environments, but they also become epistemic learners, or in Resnick's and colleagues' perspective, members of a community in which each one is accountable to the other (Michaels et al., 2008; Resnick et al., 2010). This fostering of epistemic accountability is a major product of the AWM curriculum.

Although some research examining the unique contribution of particular features of the AWM curriculum has been conducted to better understand how gains are achieved, there remain many questions to be addressed to gain a fuller understanding of how the curriculum functions at both the individual and social levels, its pedagogical implications, and its full potential. There are components of the multicomponent AWM curriculum whose 
roles have not yet been fully examined, such as the role of sameside peer collaboration in arguing against peers holding opposing views and which forms of collaboration are most effective. The roles of group work, peers' feedback, and visual aids, such as the use of different colored cards to represent the different components of an argument and their connection in the form of a sequence (argument-counterargument-rebuttal), remain to be established. Future research can also examine the specific challenges of different forms of evidence. Evidence in questionand-answer format has been shown to be more effective than traditional text (Iordanou et al., 2019); however, the effects of other forms of information, such as graphs, tables, and images (Iordanou and Constantinou, 2015), have yet to be examined. Also, what are the effects on the ability to evaluate evidence, as well as on inquiry skills, as different epistemic standards regarding evidence develop?

The AWM method, which centers around dense engagement in peer dialogue, has been proven sufficient with little in the way of adult instruction. Future work can compare the achievements of the AWM method to those of more explicit direct instruction, in particular with respect to the development of writing. Also, determining how the AWM method can be best integrated into traditional curricular subjects and at different developmental levels requires more work. The method has been implemented thus far largely by researchers, or teachers who worked in close collaboration with researchers. We should not take for granted that all teachers will be interested in learning and implementing this method. Some teachers feel uncomfortable not having full control of what goes on in their classrooms (such as talk between students that they do not hear). Some may not be convinced that student-to-student talk is productive. Therefore, future work should explore methods of professional development of teachers that enable them to try methods that may fall outside of their present comfort zones. The connection found between teachers' own argument skills and their facility in supporting students' argument skill development (Lytzerinou and Iordanou, 2020), as well as evidence of gains in pre-service teachers' argument skills following engagement in the AWM method (Iordanou and Constantinou, 2014), suggests use of the AWM method itself in the professional development of teachers. Whether such experience is sufficient to transfer to their teaching practice remains to be seen.

Another avenue for future research is to study how the curriculum can be adapted to different cultural contexts. The AWM method has been implemented with success in Eastern as well as Western cultures (Shi, 2019), with consistent findings. Yet, the question remains open of the extent to which the method will yield the same findings across a wider range of socioeconomic and academic backgrounds in non-western cultures. Even more importantly, future research is needed to investigate how the method can be adapted in order to be more suited to different cultural settings. Which are those components of the method that can or should be adapted in different cultural settings and which are essential to its effectiveness and therefore should constitute an integral part of the method across contexts? The two, Kuhn (2019) has claimed in this category, are deep engagement with a topic and dense peer-to-peer discourse.
Finally, future research can examine the transfer of gains fostered by the AWM curriculum to real-life settings and its potential to affect not only thinking but also behavior. Do the gains of the AWM curriculum transfer to whole-class discussion and to discussions, as well as individual thinking, outside of the classroom? Although there is evidence that the AWM curriculum fosters gains in intellectual values and epistemological understanding, as assessed in paper-and-pencil measures, its potential to support critical thinking in real-life contexts needs further investigation. According to Halpern (1998), “a critical thinker exhibits the following dispositions or attitudes: (a) willingness to engage in and persist at a complex task, (b) habitual use of plans and the suppression of impulsive activity, (c) flexibility or open-mindedness, (d) willingness to abandon non-productive strategies in an attempt to self-correct, and (e) an awareness of the social realities that need to be overcome (such as the need to seek consensus or compromise) so that thoughts can become actions" (p. 452). Although the connection between AWM gains and critical argumentation is clear, such gains can be further contextualized to everyday real-life decision-making. For example, does engagement in the AWM curriculum on pressing topics, such as climate change, affect individuals' immediate or longer-term attitudes and decisions on this issue? Unlike traditional school curriculum, the AWM curriculum focuses on deep engagement with contemporary social issues, such as immigration, an engagement that prepares students for engaged, active citizenship (Kuhn et al., 2019; Rapanta and Trovão, 2019). Yet, the question of whether and how engagement in the AWM method can support more responsible citizenship is an open one. In addition to more engaged, responsible citizenship, can the curriculum promote or change fundamental values, in particular the intellectual value of appreciation for the power of argument and evidence in resolving differences? Only future research can explore the full potential of the AWM curriculum and how it can further be developed to promote among the next generation the valuing of dialogue within and across societies.

\section{AUTHOR CONTRIBUTIONS}

Both authors listed have made a substantial, direct and intellectual contribution to the work, and approved it for publication.

\section{FUNDING}

CR would like to thank the ArgLab at the Institute of Philosophy of the Universidade NOVA de Lisboa. This work was funded by national funds through the FCT - Fundação para a Ciência e a Tecnologia, I.P., under the strategic project UIDB/00183/2020 and the Norma Transitória - DL 57/2016/CP1453/CT0066.

\section{ACKNOWLEDGMENTS}

The authors would like to express their appreciation to Deanna Kuhn for her thoughtful comments. 


\section{REFERENCES}

Arcidiacono, F., and Bova, A. (2015). Activity-bound and activity-unbound arguments in response to parental eat-directives at mealtimes: differences and similarities in children of 3-5 and 6-9 years old. Learn. Cult. Soc. Interact. 6, 40-55. doi: 10.1016/j.lcsi.2015.03.002

Billig, M. (1987). Arguing and Thinking: A Rhetorical Approach to Social Psychology. Cambridge: Cambridge University Press.

Blair, J. A., and Johnson, R. H. (1987). Argumentation as dialectical. Argumentation $1,41-56$.

Bova, A., and Arcidiacono, F. (2014). Types of arguments in parentschildren discussions: an argumentive analysis. J. Appl. Psycholinguistics 14, 43-66.

Britt, M. A., and Rouet, J. F. (2012). "Learning with multiple documents: component skills and their acquisition," in Enhancing the Quality of Learning: Dispositions, Instruction, and Learning Processes, eds J. R. Kirby and M. J. Lawson (Cambridge: Cambridge University Press), 276-314. doi: 10.1017/ CBO9781139048224.017

Buckland, M. K. (1991). Information as thing. J. Am. Soc. Inform. Sci. 42, 351-360.

Chinn, C. A., Buckland, L. A., and Samarapungavan, A. L. A. (2011). Expanding the dimensions of epistemic cognition: arguments from philosophy and psychology. Educ. Psychol. 46, 141-167. doi: 10.1080/00461520.2011.58 7722

Crowell, A., and Kuhn, D. (2014). Developing dialogic argumentation skills: a three-year intervention study. J. Cogn. Dev. 31, 456-496.

Felton, M. K. (2004). The development of discourse strategies in adolescent argumentation. Cogn. Dev. 19, 35-52. doi: 10.1016/j.cogdev.2003.09.001

Garcia-Mila, M., Gilabert, S., Erduran, S., and Felton, M. (2013). The effect of argumentive task goal on the quality of argumentive discourse. Sci. Educ. 97, 497-523. doi: $10.1002 /$ sce. 21057

Graff, G., and Birkenstein, C. (2010). They Say, I Say. the Moves that Matter in Academic Writing. New York, NY: W. W. Norton \& Company.

Halpern, D. F. (1998). Teaching critical thinking for transfer across domains: disposition, skills, structure training, and metacognitive monitoring. Am. Psychol. 53, 449-455. doi: 10.1037/0003-066x.53.4.449

Hemberger, L., Kuhn, D., Matos, F., and Shi, Y. (2017). A dialogic path to evidencebased argumentive writing. J. Learn. Sci. 26, 575-607. doi: 10.1080/10508406. 2017.1336714

Iordanou, K., and Fotiou, C. (submitted). Supporting Multiple-Text Comprehension Through Argumentation. Manuscript submitted for publication.

Iordanou, K. (2010). Developing argument skills across scientific and social domains. J. Cogn. Dev. 11, 293-327. doi: 10.1080/15248372.2010.485335

Iordanou, K. (2013). Developing face-to-face argumentation skills: does arguing on the computer help? J. Cogn. Dev. 14, 292-320. doi: 10.1080/15248372.2012. 668732

Iordanou, K. (2016). Developing epistemological understanding through argumentation in scientific and social domains. Zeitschrift für Pädagogische Psychologie 30(2-3), 109-119. doi: 10.1024/1010-0652/a00 0172

Iordanou, K. (in press). "Supporting critical thinking through engagement in dialogic argumentation: the case of discussing genetically modified food," in Critical Thinking in Biology and Environmental Education: Facing challenges in a post-truth world, eds B. Puig and M. P. Jiménez-Aleixandre (Berlin: Springer).

Iordanou, K. (submitted). Supporting strategic and meta-strategic development of argument skill: the role of reflection. Manuscript submitted for publication.

Iordanou, K., and Constantinou, C. P. (2014). Developing pre-service teachers' evidence-based argumentation skills on socio-scientific issues. Learn. Instruction 34, 42-57. doi: 10.1016/j.learninstruc.2014.07.004

Iordanou, K., and Constantinou, C. P. (2015). Supporting use of evidence in argumentation through practice in argumentation and reflection in the context of SOCRATES learning environment. Sci. Educ. 99, 282-311. doi: 10.1002/sce. 21152

Iordanou, K., and Kuhn, D. (2020). Contemplating the opposition: does a personal touch matter? Discourse Process. 57, 343-359. doi: 10.1080/0163853x.2019. 1701918

Iordanou, K., Kendeou, P., \& Beker, K. (2016). Argumentative reasoning. in eds J. A. Greene, W. A. Sandoval, \& I. Braten, Handbook of Epistemic Cognition. Milton Park: Routledge.
Iordanou, K., Kuhn, D., Matos, F., Shi, Y., and Hemberger, L. (2019). Learning by arguing. Learn. Instruction 63, 101-207.

Johnson, R. H. (2002). Manifest Rationality: a Pragmatic Theory of Argument. Milton Park: Routledge.

Kuhn, D. (1992). Thinking as argument. Harv. Educ. Rev. 62, 155-179.

Kuhn, D. (1999). A developmental model of critical thinking. Educ. Res. 28, 16-46. doi: $10.3102 / 0013189 \times 028002016$

Kuhn, D. (2001). How do people know?. Psychol. Sci. 12, 1-8. doi: 10.1111/14679280.00302

Kuhn, D. (2015). Thinking together and alone. Educ. Res. 44, 46-53. doi: 10.3102/ 0013189x15569530

Kuhn, D. (2018b). Building Our Best future: Thinking Critically About Ourselves and Our World. New York: Wessex Learning.

Kuhn, D. (2018a). A role for reasoning in a dialogic approach to critical thinking. Topoi 37, 121-128. doi: 10.1007/s11245-016-9373-4

Kuhn, D. (2019). Critical thinking as discourse. Hum. Dev. 62, 146-164. doi: $10.1159 / 000500171$

Kuhn, D. (2020). Why is reconciling divergent views a challenge?. Curr. Dir. Psychol. Sci. 29, 27-32. doi: 10.1177/0963721419885996

Kuhn, D., \& Zillmer, N. (2015). Developing norms of discourse. in eds L. Resnick, C. Asterhan, \& S. Clarke, Socializing Intelligence Through Academic Talk and Dialogue (pp. 77-86). Washington, DC: American Educational Research Association.

Kuhn, D., and Crowell, A. (2011). Dialogic argumentation as a vehicle for developing young adolescents' thinking. Psychol. Sci. 22, 545-552. doi: 10.1177/ 0956797611402512

Kuhn, D., and Moore, W. (2015). Argument as core curriculum. Learn. Res. Pract. $1,66-78$.

Kuhn, D., and Pearsall, S. (1998). Relations between metastrategic knowledge and strategic performance. Cogn. Dev. 13, 227-247. doi: 10.1016/s0885-2014(98) 90040-5

Kuhn, D., and Udell, W. (2003). The development of argument skills. Child Dev. 74, 1245-1260. doi: 10.1111/1467-8624.00605

Kuhn, D., Feliciano, N., and Kostikina, D. (2019). Engaging contemporary issues as practice for citizenship. Soc. Stud. 110, 207-219. doi: 10.1080/00377996.2019. 1625856

Kuhn, D., Goh, W., Iordanou, K., and Shaenfield, D. (2008). Arguing on the computer: a microgenetic study of developing argument skills in a computersupported environment. Child Dev. 79, 1311-1329.

Kuhn, D., Hemberger, L., and Khait, V. (2016a). Argue with Me: Developing Thinking and Writing Through Dialog. Milton Park: Routledge.

Kuhn, D., Hemberger, L., and Khait, V. (2016b). Tracing the development of argumentive writing in a discourse-rich context. Written Commun. 33, 92-121. doi: $10.1177 / 0741088315617157$

Kuhn, D., Shaw, V., and Felton, M. (1997). Effects of dyadic interaction on argumentive reasoning. Cogn. Instruc. 15, 287-315. doi: 10.1207/ s1532690xci1503_1

Kuhn, D., Zillmer, N., Crowell, A., and Zavala, J. (2013). Developing norms of argumentation: metacognitive, epistemological, and social dimensions of developing argumentive competence. Cogn. Instruction 31, 456-496. doi: 10. 1080/07370008.2013.830618

Larrain, A., Singer, V., Strasser, K., Howe, C., López, P., Pinochet, J., et al. (2020). Argumentation skills mediate the effect of peer argumentation on content knowledge in middle-school students. J. Educ. Psychol. doi: 10.1037/ edu0000619

List, A., and Alexander, P. A. (2019). Toward an integrated framework of multiple text use. Educ. Psychol. 54, 20-39. doi: 10.1080/00461520.2018.1505514

Lytzerinou, E., and Iordanou, K. (2020). Teachers' ability to construct arguments, but not their perceived self-efficacy of teaching, predicts their ability to evaluate arguments. Int. J. Sci. Educ. 42, 617-634. doi: 10.1080/09500693.2020.1722864

Macagno, F. (2016). Argument relevance and structure. assessing and developing students' uses of evidence. Int. J. Educ. Res. 79, 180-194. doi: 10.1016/j.ijer. 2016.07.002

Macagno, F. (2019). Coding Relevance. Learning, Culture and Social Interaction. Berlin: Springer.

Matos, F. (2021). Collaborative writing as a bridge from peer discourse to individual argumentative writing. Read. Writ. doi: 10.1007/s11145-020-10 $117-2$ 
Mayweg-Paus, E., Macagno, F., and Kuhn, D. (2015). Developing argumentation strategies in electronic dialogs: Is modeling effective? Discourse Process. 53, 280-297. doi: 10.1080/0163853x.2015.1040323

McKeough, A., Lupart, J. L., and Marini, A. (2013). Teaching for Transfer: Fostering Generalization in Learning. Milton Park: Routledge.

Mercer, N. (1995). The Guided Construction of Knowledge: Talk Amongst Teachers and Learners. Bristol: Multilingual Matters.

Michaels, S., O'Connor, C., and Resnick, L. B. (2008). Deliberative discourse idealized and realized: accountable talk in the classroom and in civic life. Stud. Philosophy Educ. 27, 283-297. doi: 10.1007/s11217-007-9071-1

Mill, J. S. (1859/1996). “On liberty," in Modern political thought: Readings from Machiavelli to Nietzsche, ed. D. Wootton (Hackett: Indianapolis/Cambridge).

Moshman, D. (2015). Epistemic Cognition and Development: The Psychology of Justification and Truth. East Sussex: Psychology Press.

Muller-Mirza, N., and Perret-Clermont, A. N. (2009). Argumentation and Education: Theoretical Foundations and Practices. Berlin: Springer.

Murphy, P. K., Greene, J. A., Allen, E., Baszczewski, S., Swearingen, A., Wei, L., et al. (2018). Fostering high school students' conceptual understanding and argumentation performance in science through quality Talk discussions. Sci. Educ. 102, 1239-1264. doi: 10.1002/sce.21471

Nussbaum, E. M. (2008). Collaborative discourse, argumentation, and learning: preface and literature review. Contemp. Educ. Psychol. 33, 345-359. doi: 10. 1016/j.cedpsych.2008.06.001

Nussbaum, E. M., Kardash, C. M., and Graham, S. E. (2005). The effects of goal instructions and text on the generation of counterarguments during writing. J. Educ. Psychol. 97, 157-169. doi: 10.1037/0022-0663.97.2.157

O’Keefe, D. J. (1992). Two concepts of argument. in eds W. L. Benoit, D. Hample, \& P. Benoit, Readings in Argumentation. Berlin: Walter de Gruyter.

Papathomas, L., and Kuhn, D. (2017). Learning to argue via apprenticeship. J. Exp. Child Psychol. 159, 129-139. doi: 10.1016/j.jecp.2017.01.013

Rapanta, C. (submitted). Can teachers implement a student-centered dialogical argumentation method across the curriculum?

Rapanta, C., and Trovão, S. (2019). Shall we receive more refugees or not? a comparative analysis and assessment of Portuguese adolescents' arguments and social representations. Pedagogy Cult. Soc. 28, 581-603 doi: 10.1080/14681366. 2019.1676298

Rapanta, C., and Walton, D. (2016). Identifying paralogisms in two ethnically different contexts at university level. Infancia y Aprendizaje 39, 119-149. doi: 10.1080/02103702.2015.1111610

Resnick, L. B., Asterhan, C. S., and Clarke, S. N. (2018). Accountable Talk: Instructional Dialogue That Builds the Mind. Geneva: The International Academy of Education.

Resnick, L. B., Michaels, S., \& O’Connor, C. (2010). How (well structured) talk builds the mind. in eds D. Pressis, \& R. Sternberg, Innovations in Educational Psychology: Perspectives on Learning, Teaching and Human Development (163194). Berlin: Springer.

Reznitskaya, A., and Gregory, M. (2013). Student thought and classroom language: examining the mechanisms of change in dialogic teaching. Educ. Psychol. 48, 114-133 doi: 10.1080/00461520.2013.775898
Schraw, G. (1998). Promoting general metacognitive awareness. Instructional Sci. 26, 113-125.

Shi, Y. (2019). Enhancing evidence-based argumentation in a mainland China middle school. Contemp. Educ. Psychol. 59:101809. doi: 10.1016/j.cedpsych. 2019.101809

Shi, Y. (2020b). Talk about evidence during argumentation. Discourse Process. 57, 770-792. doi: 10.1080/0163853x.2020.1777498

Shi, Y. (2020a). Constructed dialogs reveal skill development in argumentive writing. Read. Writing 33, 2311-2335. doi: 10.1007/s11145-020-10 045-1

Shi, Y., Matos, F., and Kuhn, D. (2019). Dialog as a bridge to argumentative writing. J. Writing Res. 11, 107-129. doi: 10.17239/jowr-2019.11.01.04

Swanson, H. L. (1990). Influence of metacognitive knowledge and aptitude on problem solving. J. Educ. Psychol. 82, 306-314. doi: 10.1037/0022-0663.82.2.306

Toulmin, S. E. (1958). The Uses of Argument. New York: Cambridge University Press.

Udell, W. (2007). Enhancing adolescent girls' argument skills in reasoning about personal and non-personal decisions. Cogn. Dev. 22, 341-352. doi: 10.1016/j. cogdev.2007.02.003

Von Aufschnaiter, C., Erduran, S., Osborne, J., and Simon, S. (2008). Arguing to learn and learning to argue: Case studies on how students' argumentation relates to their scientific knowledge. J. Res. Sci. Teach. 45, 101-131. doi: 10. $1002 /$ tea. 20213

Vygotsky, L. (1978). Mind in Society: the Development of Higher Psychological Processes. Cambridge, MA: Harvard University Press.

Walton, D. N. (1989). Dialogue theory for critical thinking. Argumentation 3, 169-184. doi: $10.1007 /$ bf00128147

Walton, D. N. (1990). What is reasoning? what is an argument?. J. Philosophy 87, 399-419. doi: 10.2307/2026735

Wilson, N. S., and Bai, H. (2010). The relationships and impact of teachers' metacognitive knowledge and pedagogical understandings of metacognition. Metacogn. Learn. 5, 269-288. doi: 10.1007/s11409-0109062-4

Zavala, J., and Kuhn, D. (2017). Solitary discourse is a productive activity. Psychol. Sci. 28, 578-586. doi: 10.1177/0956797616689248

Zillmer, N., and Kuhn, D. (2018). Do similar-ability peers regulate one another in a collaborative discourse activity? Cogn. Dev. 45, 68-76. doi: 10.1016/j.cogdev. 2017.12.002

Conflict of Interest: The authors declare that the research was conducted in the absence of any commercial or financial relationships that could be construed as a potential conflict of interest.

Copyright (C) 2021 Iordanou and Rapanta. This is an open-access article distributed under the terms of the Creative Commons Attribution License (CC BY). The use, distribution or reproduction in other forums is permitted, provided the original author(s) and the copyright owner(s) are credited and that the original publication in this journal is cited, in accordance with accepted academic practice. No use, distribution or reproduction is permitted which does not comply with these terms. 
OPEN ACCESS

Edited by:

Antonio Bova,

Catholic University of the Sacred

Heart, Italy

Reviewed by:

Arianna D'Ulizia,

National Research Council (CNR), Italy

Emmi Koskinen,

University of Helsinki, Finland

*Correspondence:

Marilena Fatigante

marilena.fatigante@uniroma1.it

Specialty section:

This article was submitted to

Language Sciences,

a section of the journal

Frontiers in Psychology

Received: 05 February 2021

Accepted: 22 April 2021

Published: 03 June 2021

Citation:

Fatigante $M$, Zucchermaglio $C$ and Alby F (2021) Being in Place: A

Multimodal Analysis of the

Contribution of the Patient's

Companion to "First Time"

Oncological Visits.

Front. Psychol. 12:664747.

doi: 10.3389/fpsyg.2021.664747

\section{Being in Place: A Multimodal Analysis of the Contribution of the Patient's Companion to "First Time" Oncological Visits}

\author{
Marilena Fatigante*, Cristina Zucchermaglio and Francesca Alby \\ Department of Social and Developmental Psychology, Sapienza University of Rome, Rome, Italy
}

Companions to medical visits have been alternatively viewed as members who "support" or "inhibit" and "interfere" with the doctor-patient interaction. One way of looking at the companions' contribution to medical visits is by coding roles or functions of their communicative behavior. Our paper aims at reconsidering these findings and analyzing how the companion participation is a local and sequential accomplishment, changing from time to time in the consultation. The paper relies upon an overall collection of 58 videorecordings of first oncological visits. Visits were conducted in two different hospitals, one of which a University hospital, and by different oncologists, including both senior professionals and (in the second setting) medical students in oncology. Visits were fully transcribed according to the Jeffersonian conventions and authors examined the transcripts and video according to the methodology of Conversation Analysis. The aim of the paper focused on how patient's companions orient and contribute to the accomplishment of the different aims and activities at different stages of the visit as an institutional speech event. The multimodal analysis of turns and actions (such as, gaze shifts, prosodic modulation, bodily arrangements), and the close examination of the sequential and temporal arrangements of companions' and their co-participants' turns revealed that companions finely attune to the multiparty framework of the encounter and the institutional constraints that govern the oncological first visit. Overall, results show two relevant features: that companions act as to preserve the doctor-patient interaction and to maintain the patient as the most responsible and legitimate agent in the interaction; that companions' contributions are relevant to the activities that sequentially unfold at different stages in the consultation (e.g., history taking, problem presentation, treatment recommendation etc.). The study complements earlier findings on the companion's roles, showing how these are highly mobile, multimodal and multiparty accomplishments, and they are tied to the specific contingencies of the visit. The results solicit to consider the value of multimodal analysis in understanding the complexity of multiparty communication in medical setting, and make it usable also in medical education.

Keywords: doctor-patient communication, oncology, companion, conversation analysis, multimodality, participation, Italy 


\section{INTRODUCTION}

Communicating about cancer poses a huge burden upon patients, due to the high complexity of the information that they have to process (Davis et al., 2002; Han et al., 2011), the intense and potentially frightening emotions that may arise, primarily in the first stages of apprehending the illness (Nail, 2001; Singer, 2018), the variety and relevance of cognitive and decision making processes that have to engage, including the consideration of treatment options, the assessment of benefits and risks, the practical issues related to the beginning of the treatment (Epstein and Street, 2007; Fatigante et al., 2020). In this context, the presence of the patients' companions to the visit can have a strong impact on various aspects of doctorpatient communication, including doctors' and patients' chances to understand each other and/or attune to each other (Pino et al., 2020), and to engage in decision-making (Hubbard et al., 2010; Laidsaar-Powell et al., 2013; Laryionava et al., 2018).

Overall, companions present in the oncological visit-who are most often family members (Lamore et al., 2017) are reported to facilitate the communication between the doctor and the patient and provide instrumental and emotional support to the cancer patients (Ellingson, 2002; Del Piccolo et al., 2014). However, they are also reported as being obtrusive, and inhibiting patient's participation, in settings where the patient is elderly or vulnerable, as well as, at advanced or terminal stages of the patient's illness (Mazer et al., 2014; Pino and Parry, 2019). Relevant to our investigation, is the mention that existing studies on the topic mostly rely upon the ascription of specific individual actions and behaviors of the companions to preassigned role categories in coding systems (cf. Street and Gordon, 2008). Verbal behavior and, particularly, self-initiating moves such as, questions, are taken as the primary indicator of their participation (cf. Street and Gordon, 2008; Del Piccolo et al., 2014); less attention is dedicated to the way they engage - and are engaged by their co-participants- through other communicative modalities (e.g., by gaze, gestures, and actions). Further, no distinction is made with regards the moment in the visit where the companion intervenes, overlooking that the medical visit is a sequential, institutional event (Drew and Heritage, 1992) that temporally and orderly unfold through multiple stages (Robinson and Stivers, 2001; Robinson, 2003).

Our work adds to existing literature (Ellingson, 2002), confirming that companions play several functions in support of the patient throughout the visit. However, our study uniquely contributes to this field of research by showing that "roles" are in fact highly mobile accomplishments, subject to the coparticipants' responses and ratification; further, we show that the companions' (either discourse and bodily) moves are deeply tied to the specific stages and institutional aims of the visit, and it is only in light of the specific aims and constraints of the visit's stage that the companions' (as well as the other participants') actions can be relevantly interpreted.

We take the example from the oncological setting as an opportunity to indicate that the close analysis of participants' publicly visible and reflexive actions in talk (including not only discourse but gaze, gestures, material arrangements of artifacts and tools available to them) is an extremely rich and viable methodology in psychological research interested in the study of communication in sensitive environments.

\section{BACKGROUND}

There exist extensive evidences that family members, friends, or acquaintances who accompany the patients at the medical visit facilitate the communication between the doctor and the patient and overall play a supportive role, particularly as complex information are delivered and may be difficult for the patient to comprehend (Laidsaar-Powell et al., 2016).

Companions appear to be involved more with elderly patients, patients with increased needs (such as, in pain or in advanced chronic stage of the illness) (Clayman et al., 2005; Ishikawa et al., 2005; Wolff and Roter, 2008; Jansen et al., 2010; Legare et al., 2014; Wolff et al., 2017) or minority patients (Mitchell et al., 2019).

In a 2002 article on interdisciplinary oncological visits with elderly patients, Ellingson identified several roles of the companions, including: aiding in memory, providing emotional support, transcribing information for the patient, aiding in decision making, providing companionship, providing elaboration and context of the patient's response, advocating reasons for patient, and interpreting the doctors' words for the patient.

Other studies indicated that the presence of the companion can generate ambiguities and tensions, due to the fact that companions may sometimes censor the patients' voice, acting as if they were not present (Mazer et al., 2014 call this acting as a pseudo-surrogate of the patient) and display more involvement than desired (by patients) or expected (by doctors) in decision making (Shepherd et al., 2007; Eggly et al., 2013; Laidsaar-Powell et al., 2013, among others).

In cancer visits with newly diagnosed patients, authors have particularly considered the extent to which companions ask questions (Eggly et al., 2006, 2011; Street and Gordon, 2008; Del Piccolo et al., 2014), taken as an indicator of active engagement and support to patients' needs for information.

With particular regards to a collection of Italian visits with (breast cancer) patients, Del Piccolo et al. (2014), reported that most of the (breast cancer) patients' companions in their study helped the patient report or ask for information (e.g., completing the patient's reports, checking or validating the completeness of information), while not inhibiting the patient's involvement in the interaction. In line with what found by Street and Gordon (2008), this study also reported that the companion does not significantly affect the degree of verbal engagement by the patients.

These observational studies, although based on audio- and video- recorded interactions, have subsumed their results via coding schema, that is, systems which assign pre-defined values to contributions, namely, utterances or, statements. Basing on pre-assigned categorization of interactional "moves," authors have, in turn, identified different "roles" to the companion, such as, that of "passive observer," advocate, partner or "shared role" 
(Street and Gordon, 2008; Del Piccolo et al., 2014), surrogate or pseudo-surrogate of the patient (Mazer et al., 2014).

Whereas, coding schemas help differentiate among several diverse conducts and positions that may be enacted by the companion in relation to the patient, they suffer from two limitations: (1) they consider the actor's behavior, that is, an individual, self-contained unit, almost independent from the sequential context, as the target of analysis and (2) they assign the target behaviors to pre-assigned labels, based on the researcher's hypotheses. As such, they do not capture the interactional details, unfolding via verbal and non-verbal resources and the fine coordination among them, which the participants in any ordinary or institutional setting attend to. Focusing on the sequential environment in which participants' turns are allocated (Schegloff, 2007), studies conducted within Conversation Analytic paradigm have demonstrated that the interlocutors' positions in a conversation are highly mobile and always open to negotiation by co-present parties, particularly in a multiparty encounter (Goodwin, 1979, 1984, 1987; Schegloff, 1995, 2000; Goodwin and Goodwin, 2004; among others). Also, to look at how participants coordinate verbal and non-verbal resources is crucial. Despite Mazer et al. (2014) attempted to study the conversational context of the companion's utterances, they only looked at conversational turns (limited to 2), respectively, preceding and following companion statements, and they did not take into account non-verbal, multimodal cues, which have been demonstrated in other contexts as relevant signals for participants to negotiate their initiative at talk (Goodwin, 2000; Mondada, 2007; Stivers, 2008; Ruusuvuori and Peräkylä, 2009).

To date, only few studies have examined companions' initiatives as sequential accomplishments.

Explicitly grounding on Conversation Analytic principles, Pino and Parry (2019) examined the companions' contribution on talk in visits with (terminally ill) patients. In these cases, the sensitivity of topics related to the end-of-life and the pervasive worries that affect both the patients and their significant others, appear to solicit a more active engagement of the companions. The in-depth, sequential analysis conducted by the authors show that, more than binding and simply replacing the patient's opportunity to respond to certain doctor's question, companions' contributions are managed as to sequentially open the relevant conversational slot for the patient to produce a request by herself, i.e., about life-expectancy estimate. Conversation Analysis is also applied by another study by Pino et al. (2020) to analyze healthcare providers' responses to companions' turns in the context of palliative care. Authors show that healthcare providers precisely monitor the sequence of patient- companion's turns in order to avoid to be heard as siding with one or another, and to express a position on an independent, expert basis.

Drawing on the literature examined so far, this article applies a conversation-analytic methodology to the analysis of the contribution of the patient's companion in first oncological visits. These are visits, which occur between cancer patients and oncologists who meet together for the first time, after the patients have already got the cancer diagnosis, and they have also often undergone surgery for that. Basing on the diagnostic assessment, which is routinely reviewed in the visit, these encounters are primarily aimed at presenting and considering the treatment options for the patient, in order to get to a decision.

The study aims at providing an in-depth examination of the companions' participation in the oncological visit. In contrast to coding participants' single behavior, we examine how actions are allocated in sequences and we analyze participants' turns (either verbal or non-verbal) as the result of complex sequential, multiparty arrangements by all participants. We also take into account the placement of the companions' contribution in the multi-staged structure of the visit. To our knowledge, no study has considered the companions' contribution in relation to the particular structure and the specific institutional tasks and activities that this kind of visit (see Fatigante et al., 2021) involve. Further, we include multimodal cues (such as, gaze and gaze shifts, posture, gestures, modulation of the tone of voice) as essential to indicate how the participants orient to the talk in progress and convey their own understanding of their actions.

\section{MATERIALS AND METHODS}

\section{Methodology}

The study grounds on the methodological framework of Conversation Analysis (CA). Conversation Analysis is a qualitative method of the analysis of interaction, which uniquely dedicates attention to the sequence of turns and actions, considered as the site for the production of participants' mutual intelligibility (Sacks et al., 1974; Heritage, 1984a).

Accordingly to CA methodology, members' contribution to the talk are only comprehensible within the sequential environment in which the turn was built. Members' contributions are mutually related in minimal sequential units called "adjacency pair," such as question-answer, greeting pairs, and other sequences such as invitation-response, assessments pairs, formulation-response (confirmation or rejection). In all these conversational pairs, the first pair part instantiates the expectation that the second pair part of a relevant "type" will follow. The absence of the second pair, however possible, will be treated by participants as "relevantly absent," and can mobilize repair moves (Schegloff et al., 1977) by participants (such as, a re-formulation of the question in the absence of a response) in order to re-establish mutual intelligibility. Not only verbal strings of talk but also, multimodal cues (such as, gaze and gaze shifts, posture, gestures, modulation of the tone of voice) are captured and analyzed by Conversation Analysis as relevant resources by the participants to orient to the talk in progress and reach mutual understanding of their actions. For this reason, transcription is essential. A specific system of notational symbols, named as Jeffersonian system after Gail Jefferson who first implemented it (Jefferson, 2004) ensures that formal aspects of talk production, both intonational and sequential, upon which the analysis is based are made available in the transcripts, constituting the public evidences supporting the validity of the analysis.

The use of Conversation Analysis reveals particularly fruitful as a methodology capable of showing in detail how participants adjust interactional resources as to sophisticatedly pursue 
different activities and tasks, albeit delicate and complex, in the medical interaction (Heath, 1986; Heritage and Maynard, 2006).

\section{Data Collection}

The collection of videorecordings of first visits took place in the Oncology Departments (Day-Hospital) of two different settings: a medium size hospital (Site 1) and a large University hospital (Site 2) both located in Rome, Italy. The overall corpus counts 58 videorecorded visits, 33 visits collected in Site 1, and 25 collected in Site 2.

Prior to the collection of videorecordings, ethnographic fieldwork was conducted, in order to consider organizational features of the context, e.g., agenda of the visits, availability of the doctors, spatial characteristics of the waiting room, workflow of the visits across the day. Fieldwork lasts overall 2 months, and it comprised taking notes, collection of photographs and formal and informal interviews with the doctors. All this material was also useful to assess appropriate places and times in which to recruit candidate patients and present them the informed consent to the study. Consent was always taken the same day of the patient's appointment with the oncologist.

\section{Ethics}

The study received approval from the Ethical Committee of both hospitals. Written informed consents were obtained from all participants (doctors, patients, and patients' companions). Patients (and companions) were approached and offered the informed consent during their waiting time prior to the visit. Upon the patient's and companion's agreement, a video-camera was positioned in the visiting room. Video-recordings were then safely stored and used for analytical purposes. Images were used, when useful to illuminate how participants' actions and gestures were arranged in relation to talk, as to produce a certain outcome. Due to issue of privacy, images were blurred in order to avoid participants' facial recognition.

\section{Participants}

Participants included 2 senior oncologists (one in each site, with more than 35 years of experience in that specialty) and 4 junior residents in oncology (3 females and one male) in Site 2. In Site 1 , all the visits were conducted by the senior oncologist; in Site 2, visits were conducted by resident only $(9 / 25)$ and by the senior oncologist and one of the resident in the rest of the visits.

Fifty-eight patients and 46 companions participated in the study.

Most patients in the two data corpus are women (80\%) who received a breast cancer diagnosis (77\%). Their average age (across the two corpora) is 55 years, ranging from 23 as for the youngest to 81 as for the oldest patient. 10/58 were foreign patients, able to comprehend Italian although they did not speak fluently and, in one case, the patient was not able to speak Italian at all.

\section{Transcription}

Videorecorded visits were fully transcribed according to Jeffersonian conventions (Jefferson, 2004), which account for both prosodic and sequential formal aspects of turn production.
The transcription of speakers' verbal turns were complemented with the annotation of multimodal aspects (such as, gaze) and bodily actions (Mondada, 2018), co-occurring with the speaker's or co-participants' words. Multimodal markers were taken into account as powerful resources that signal, particularly in multiparty conversations, changes in participation framework (Goodwin, 1984; Goodwin and Goodwin, 2004) and shifts in participants' orientation to the activity in progress (Mondada, 2007), or their mutual understanding and alignments. Names and other references to places (e.g., hospitals) have been modified into fictional ones. Transcription symbols are provided in the Appendix A.

\section{Data Analysis}

The conversation analytic literature now widely available on medical discourse (Heritage and Maynard, 2006) has shown how visits are organized in a particular fashion: they develop accordingly to a series of stages that develop sequentially and orderly, although this order can sometimes admit variations (Robinson and Stivers, 2001; Robinson, 2003; Koenig, 2011; Fatigante et al., 2021). Accordingly, we examined companions' turns in relation to the specific stage of the visit in which they occurred, analyzing whether and how they supported its related aims and activities.

As mentioned, we also dedicated a particular attention to the sequential and temporal management of multimodal cues (such as, shifts in gaze and posture, gestures, modulation of the tone of voice) in the construction of participants' turns and we made available in the transcripts those aspects, which were treated as relevant by the participants to orient to the talk in progress and reach mutual understanding of their actions.

As for the analytic aim of this paper, transcription of each visit was read independently by each author, who sorted out all instances in which the companions contributed to the accomplishment of the different stages of the visits (cf. Table 2; as for how stages were identified, see Fatigante et al., 2021). We removed from this analysis one visit only, in which the patient could not speak Italian at all. We considered that the specificity of the companion's role as language broker in this case (however she was not a formal interpreter) made the visit and the participants' arrangements in turn taking much different from the others and required examination in a different paper.

In line with the qualitative methodological perspective adopted, we focused on the sequential development of the conversational excerpts and paid attention to what the companion's turn (either expressed by discourse moves or bodily resources) responded, and what "next" relevant action it originated (Sacks et al., 1974).

\section{RESULTS}

A companion was present in $38(66 \%)$ of the visits of our data corpus. Accompanied visits rate higher in Site 1 (75\%), in which the mean age of all patients is also higher (60.9 vs. 50.5$)$, while in Site 2 the number of accompanied and unaccompanied visits is similar (52\%). 
TABLE 1 | Relationship of the companion to the patient.

\begin{tabular}{lc}
\hline Family members & $34(73.9 \%)$ \\
Friends/acquaintances & $11(23.9 \%)$ \\
Paid caregiver & $1(2.2 \%)$
\end{tabular}

Notably, among patients older than 75 years old (6 in both corpora), $83 \%$ were accompanied. As regards the gender of accompanied vs. unaccompanied patients, female cancer patients tend to be accompanied more than their male counterparts.

The overall number of companions is 46 , a figure that exceeds the number of patients, indicating that some visits included more than 1 companion. Table 1 describes the relationship that the companions had with the patient.

Family members included the patient's spouse for the most part (53\%), an adult child for a smaller proportion (28\%), a sibling and one (or both) parents of the patient.

\section{The Stages of the Oncological Visit}

In order to delineate a few quantitative coordinates of the visit as a spatio temporal communicative event, we first provide some background information about the average length of the visits and its different stages. Visits in the corpus last $27.5 \mathrm{~min}$ on average, with a maximum length of $40^{\prime}$ and a minimum of $10^{\prime}$, a feature that varied in relation to the time pressure that participant oncologists experienced in the specific day of the data collection (apprehended by the researchers' field notes and participant observation). Daily timetables filled with first oncological appointments generally spanned between 7.30 a.m. and $1.30 \mathrm{p} . \mathrm{m}$. and they included 7 visits per day on average, a number that sometimes varied, to reach up to 12 visits.

As regards the structural organization of the oncology first visits, prior work on this data corpus has delineated different stages (Zucchermaglio et al., 2016; Fatigante et al., 2021), each aimed at performing a different and institutional activity of the visit, and thus also implying a different opportunities, rights, and responsibilities (or, status) of participation (e.g., answering doctor's questions vs. listening to his explanations; cf. Heritage and Maynard, 2006).

In the table below we provide a brief overview of each stage.

Parentheses \{\} indicate that included stages are not always present.

Previous analyses (Fatigante et al., 2021) have also evidenced the relative length of each stage in this visit. So, we mention that the longest, most prominent stage in this visit is the Outline of future actions (19\% of the visit total length), immediately followed by the Treatment recommendation stage (18\%) and the Cancer diagnostic assessment (14\%). The stage of Cancer problem presentation stands for the $13 \%$ of the visit total length, with the other stages rating almost equally (Openings 9\%, History taking $8 \%$, Closings 8\%; when present, Physical examination rates $5 \%$ ).

These percentages inform us that the most significant activities in this kind of visit are those, in which the oncologist delivers information, explanations, recommendations and advice to the patients and companions. This feature would support the evidence, gathered since pioneering research on medical interaction (Hall et al., 1987; Bensing and Dronkers, 1992; Emanuel and Emanuel, 1992), for which the instrumental dimension of talk exceeds the socio-emotional one in this kind of setting (cf. also Eide et al., 2003).

Given this picture, which would see the doctor mainly providing information, and the patient (correspondingly) in the position of his main addressee, what the companion can contribute to the development of such an event, and to the different activities that unfold therein?

The excerpts that follow were chosen and selected as particularly clear illustrations of the ways in which the companion contributes differently to the unfolding of the sequence of activities of the visit (see Table 2). We will now provide some examples extracted from each different stage of the visit, which are particularly representative of the strategies, carried out by means of verbal and non-verbal resources, used by the companions to engage in conversation.

\section{Companion's Participation in Opening the Visit: Engaging in Small (Sociable) Talk}

However routinized, openings in interaction imply a complex coordination by interactants (Schegloff, 1968; Duranti, 1997): particularly in institutional exchanges such as a medical visit, members have to concurrently and timely manage their entrance onto the official business of the encounter, thus rapidly traversing each other's self-presentation. Despite this, we have found that visit openings do not only include greetings but also small talk sequences (Laver, 1975; Coupland, 2000; Holmes, 2000). These are sequences, often found in correspondence to "boundaries" of the interaction (Laver, 1975), which are not necessary to the instrumental task of the interaction, but they help participants to establish a common ground and ultimately test that they will be mutually cooperative partners (Maynard and Hudak, 2008).

In the next excerpt, the oncologist (Site 1) is filling the patient's record with his personal data: he has already asked the patient's name and what his job is. The oncologist's inquiry reveals that the patient works as seller of a coffee company, whose brand the oncologist knows. This originates a sequence of small talk.

The initial configuration of the participants' bodies and gaze is the following (see picture): the oncologist is writing down the patient's data, the patient sits with a folder and his jacket on his lap, the companion sits at the left of the patient, with her jacket on.

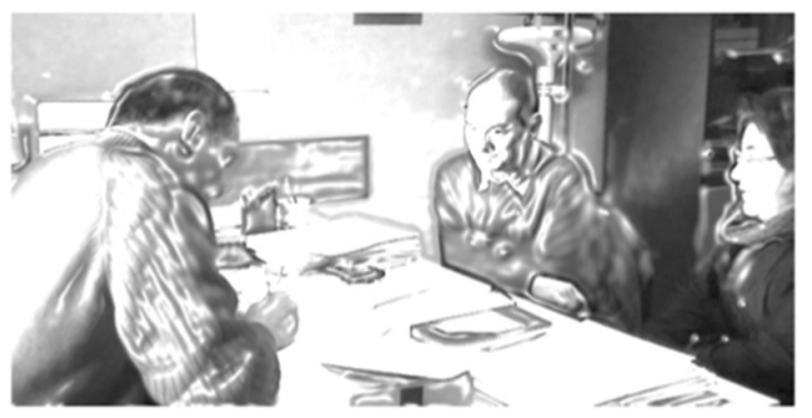




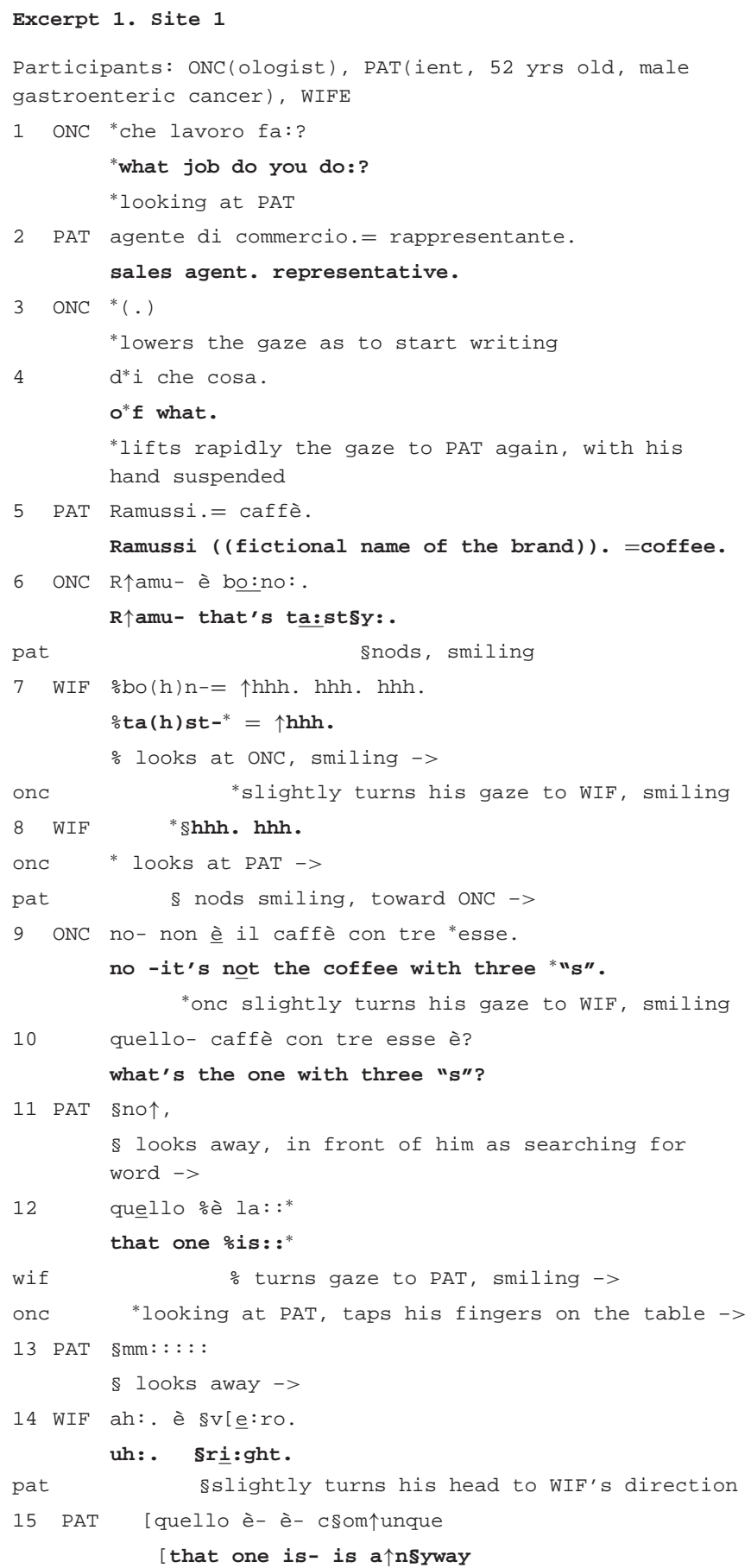

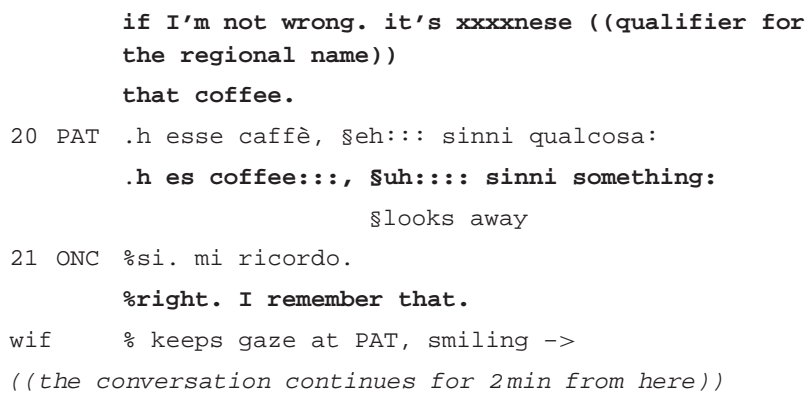

This excerpt shows how the companion monitors the oncologist's action since the beginning, while the oncologist writes down the information and continues as the oncologist asks the patient about his job (lines 1-6). The wife enters the conversation, when the oncologist pronounces his assessment of the coffee as "tasty" (line 6): here, the patient's wife immediately lifts her gaze to him, she starts repeating his same assessment and infuses it with laughter, that she continues through subsequent lines. By starting to repeat the doctor's assessment, the wife sides with him (Goodwin and Goodwin, 1992). Furthermore, by initiating and then continuing to laugh (lines 7-8), she self- candidates as an affiliate audience of the doctor's ironic performance, which in turn is indexed by the doctor's choice of the word "bono" (this comes from the Roman jargon and replace the Italian "buono," meaning "good). By using that term, the doctor is trespassing his identity from the institutional one, of oncologist, to an informal one of a common inhabitant and speaker of Rome (see Ochs and Schieffelin, 1989, for how language indexes geographical provenience). So doing, the patient's wife aligns with the doctor twice: agreeing with his assessment and affiliating with the ironic key implicit in his formulation (on laughter and affiliation, cf. Jefferson et al., 1987; Glenn, 2003).

From the positions taken by the participants relatively to the videocamera, we cannot make sure whether the oncologist is "responding" to the wife's turn. Yet, it is visible that he slightly moves his head toward her (corresponding to line 7), thus indicating that he acknowledges her initiative.

In the subsequent turns, the oncologist continues to question the patient's "expertise" upon the coffee brand, while the companion takes the position of audience to the talk in progress. She turns to her husband as he manifests uncertainty in the word searching (line 12), and she only utters a formulation later on (line 14: "uh: right"): this, tough, does not impose any constraint upon the others' contributions. By acknowledging the evidence, recalled by the doctor, that another brand exists, which might be confused with the one they are discussing, she communicates that she is fully "on topic," but she does not recruit attention by any of the participants.

Despite this, her presence is visibly acknowledged and used by the patient. Overlapping with the wife's intervention (line 14), the patient turns his gaze in her direction.

In a 1987 remarkable paper "Forgetfulness as an interactive resource," Charles Goodwin showed that participants engaging in word search use gaze as a "framing device" capable of converting what would otherwise be a private thinking activity onto a "social 
TABLE 2 | Stages of the oncological visit (Fatigante et al., 2021).

\begin{tabular}{|c|c|}
\hline Stage & Definition \\
\hline Openings & $\begin{array}{l}\text { It includes greeting sequences, sequences of small talk that bridge the participants' official entrance into the business of the visit, } \\
\text { followed by identification sequences (such as, the request and registration of the patient's name and address). It is routinely } \\
\text { accompanied by the opening and writing of the patient's record. }\end{array}$ \\
\hline History taking & $\begin{array}{l}\text { It includes the oncologist's activity of questioning regarding the clinical history of the patient (including present and past illnesses, } \\
\text { surgical interventions, current pharmacological treatments, etc.), beyond the recent cancer diagnosis. It is relevant in order for the } \\
\text { oncologist to assess cancer comorbidities, useful to plan a treatment recommendation that has no harmful consequences for that } \\
\text { particular patient (Zucchermaglio et al., 2016; Pino et al., 2021) }\end{array}$ \\
\hline Cancer problem presentation & $\begin{array}{l}\text { This stage includes the patient's description and narrative regarding the current cancer problem: when it has been discovered, how, } \\
\text { when the patient has undergone surgery etc. It is quite short in Site 1, where the oncologist only asks how the patient discovered it } \\
\text { and then asks the patient to see the documents; in Site 2, the patients and companions are left more time to build narratives of the } \\
\text { realization of the tumor and events that follow that, which can develop across several turns }\end{array}$ \\
\hline Cancer diagnostic assessment & $\begin{array}{l}\text { Also corresponding for the most part to what in oncology is referred to as the "staging" of the cancer, the diagnostic assessment } \\
\text { stage includes the examination of tests brought by the patient (mammography, ultrasounds, surgical reports, and primarily the } \\
\text { histological exam) and the explanations given to the patient about the figures and tests }\end{array}$ \\
\hline Treatment recommendation & $\begin{array}{l}\text { It comprises the presentation and discussion about the treatment options. It includes even lengthy and highly complex explanations } \\
\text { about the risks and benefits of the treatment. It also sometimes, but not routinely, include reference to collateral effects and } \\
\text { prognostic assessments. }\end{array}$ \\
\hline \{Physical examination\} & $\begin{array}{l}\text { Physical examination may occur either to aid in the diagnosis of the cancer size, location or progression or to assess the } \\
\text { post-surgical scar on patient's body }\end{array}$ \\
\hline Outline of future actions & $\begin{array}{l}\text { It comprises the oncologist's verbal recommendation and written prescriptions of next appointments, exams; it also includes } \\
\text { instructions about the practical management of the illness (e.g., changes in work agenda, whom to call if the patient feel sick after the } \\
\text { treatment etc.) }\end{array}$ \\
\hline Closings & $\begin{array}{l}\text { It is marked by the participants' orientation to the closing of the official business of the visit, such as, closing, removing documents } \\
\text { from the table and folding them, acknowledgments, greeting sequences }\end{array}$ \\
\hline
\end{tabular}

activity, one that parties other than the speaker can actively participate in" (p. 118). Something similar happens here. Yet, here the patient does not fully complete the action of gazing at her wife. His gaze remains mid-way, something, that allow him to maintain availability to the oncologist only. On the other hand, the wife restrains her participation in such a way, as to not inhibit the interaction flow between the patient and the doctor.

However, small talk sequences may appear quite inessential to the institutional unfolding of the main tasks of the visit, their analysis helps to highlight and anticipate what will reveal as the main feature exhibited by the companions' contribution in the visits of our corpus: the placement of their turns in a position, which manifests their effort to avoid taking the floor and engaging directly in the main stream of interaction with the doctor.

Via her almost inaudible, interstitial comments, the companion projects her role as ancillary and "appended" to the current speaker's contribution. We will find examples of this in several other parts of the visit.

We show another excerpt, which has different background features from the previous one, and that, however, leads to similar findings.

The next excerpt occurs at $0^{\prime} 40^{\prime \prime}$ from the patient and companion's entrance in the oncological cabinet. The companion, who is a doctor herself, and the oncologist have just found out that they already met before (in a medical conference) and the companion is telling him some details about the site where she works. As she stops, the oncologist asks:

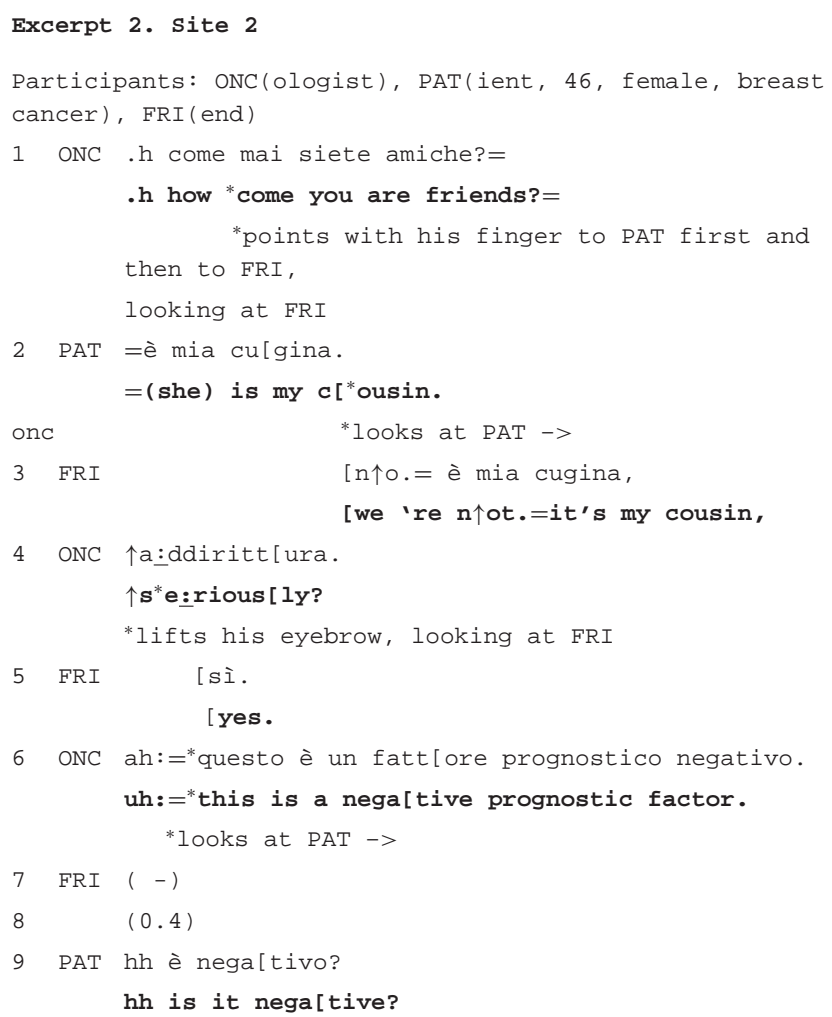


10 ONC

[parenti di medici, pare(h) [nti di psi(h)logi, =

[doctors' relatives,

psyc(h)ologists' relat(h)ives,

*looks at PAT ->

11 FRI e si

oh right

$12 \mathrm{ONC}{ }^{*} \mathrm{e}=\mathrm{e}=\mathrm{e}$ è ' $\mathrm{n}$ macello. [.hhh no scherzo.

$*^{*} i t=i t=i t^{\prime} s$ a mess. [.hhh I'm joking.

*turns gaze to the computer at his left

13 PAT $\$\left[v^{2} a b b e^{\prime}(h)\right.$ è un supporto morale.

\$[well (h) it's a moral support.

slifting her shoulders and looking upward

Despite the oncologist recruits both the companion and the patient in asking what is the current relationship between them, and notwithstanding the fact that he was engaging with the companion, and not the patient, in the previous conversation, the companion does not respond to his question. Rather, she leaves the floor to the patient, and only adds her contribution after her, coming as "second." This poses one of the most common feature of our analyses. The companions' placement of their turns after the patient's one, also when they were fully involved in talk and they would have chance to take the floor.

It is also visible in the junctures of the turns the oncologist's effort to concurrently look at one or the other. At line 4, the oncologist responds looking at the companion, while immediately after (line 6), he shifts gaze and maintains it on the patient, who accounts by telling him that the companion she brought is a "moral support." Therefore, it is clear from the beginning of the visit that the presence of the companion requires that all participants make efforts to attend to the multiparty framework of this encounter.

\section{The Companion's Contribution to the History Taking. Monitoring the Patient's Participation}

Facilitating information exchange has been documented by studies on companions' and, particularly, family members' contribution to medical visit (Wolff and Roter, 2011). In history taking, this impies that the companions help the patient recall information (Jansen et al., 2010), provide directly information to the doctor, check and validate the patient's report, solve and help repair the doctor-patient's mutual understanding. But how do they do so? We have consistently found in our corpus that, even in cases in which the companions take turn to address the doctor themselves, they pay attention to maintain the patients as the legitimate tellers of their medical history.

Excerpt 3 shows one instance of this practice. Here, the companion contributes in the accomplishment of the activity of recollecting the current medical history of the patient, unfolding through the anamnestic stage. The patient is addressed by the doctor with a question about other non-oncological relevant illnesses.
Excerpt 3. Site 1

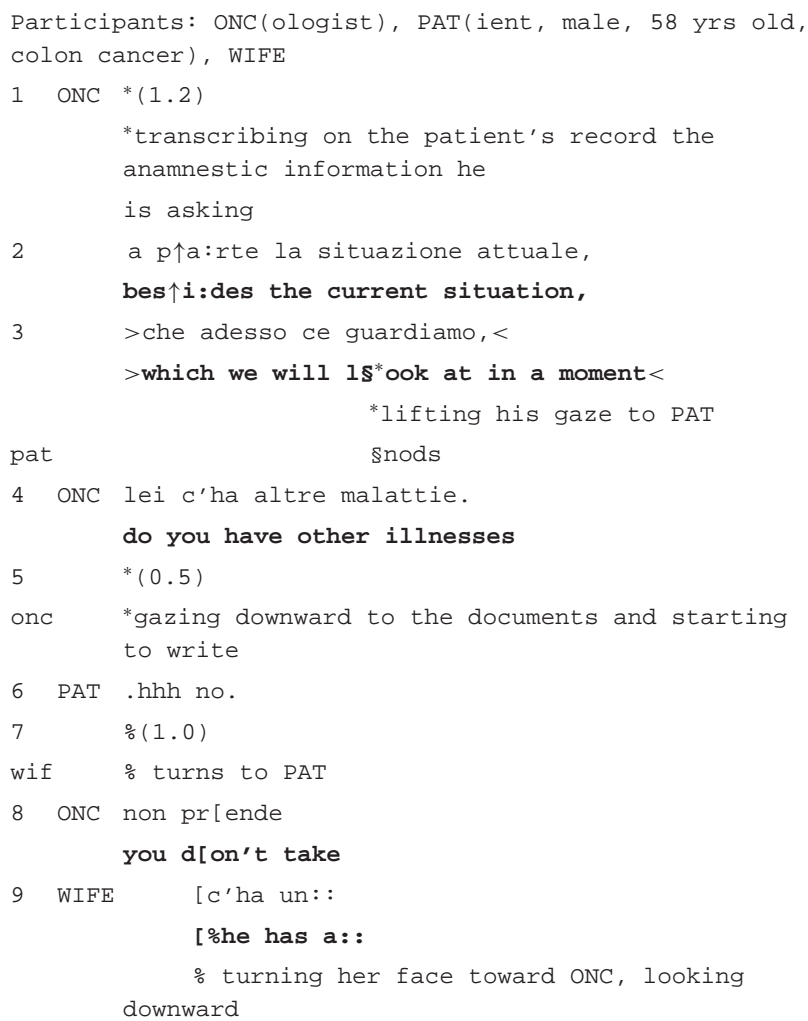

As visible in the transcript, the companion (i.e., wife) gazemonitors the patient's response (line 7 ). She only intervenes and prompts the patient's response at line 11 , when it is clear that the information provided by the patient, denying the presence of other medical conditions beside the cancer, is about to be registered as valid and permanent (for the oncologist is writing the patient's response on the record). 
It might also be noted that the companion revises the patient's answer in such a way (whispering, and avoiding to gaze to any interlocutor in particular, lines 11 and 14) that her turn can be heard as not fully claiming public "visibility," whereas, it can be captured as a "prompt" by the patient, who produces in fact a change-of-state token (Heritage, 1984b) and starts to repeat (line 17) the name of the illness, incorporating his wife's suggestion.

So doing, while powerfully influencing the content of the response to the doctor's question, the companion has shielded her role of author of the report.

The companion only looks at the oncologist (line 17), after this one has explicitly solicited the patient himself to report information, and by adding an informal token ("come on," line 15) he ironically treats him as someone who has ostensibly and consciously resisted to tell him (see Craven and Potter, 2010 and Pauletto and Fatigante, 2015 for the use of, respectively, "come on" and "dai" in Italian). By smiling (line 17), the wife conveys her ironic assessment about her husband's inattention and invites the doctor to align with her on this (Jefferson, 1984; Glenn, 2003). The doctor, indeed, maintains his gaze on the patient and will pursue from him, not the companion, the report of the glaucome illness (lines not reported).

In sum, the companion's contribution here was essential in completing and validating anamnestic information. These are usable to assess co-morbidity, which would otherwise be lost, with potential harmful consequences for the patient's cure. Notwithstanding this, it is the patient who is recognized as primary reporter and ratified by both the doctor and the companion as the most relevant addressee and character of the institutional activity of history taking.

We also found evidences of this preference even in cases, where the companion is explicitly ascribed the role of talking "on behalf" of the patient. This is clearly the case, when the patient is not able to speak for himself, due to some impediments related to illness or, to language issue.

The next example provides such a case. The sequence develops during the history taking stage. The patient, a 70 years old man, has undergone a surgery on his tongue and is not so much able to speak fluently. He has identified explicitly the wife as talking on behalf of him at the very beginning of the interaction. Notwithstanding this prior agreement, we observe that: (1) the doctor continues to look at the patient when he asks questions and (2) the wife gazes at the patient as to check for accuracy of information, despite she has access to that.

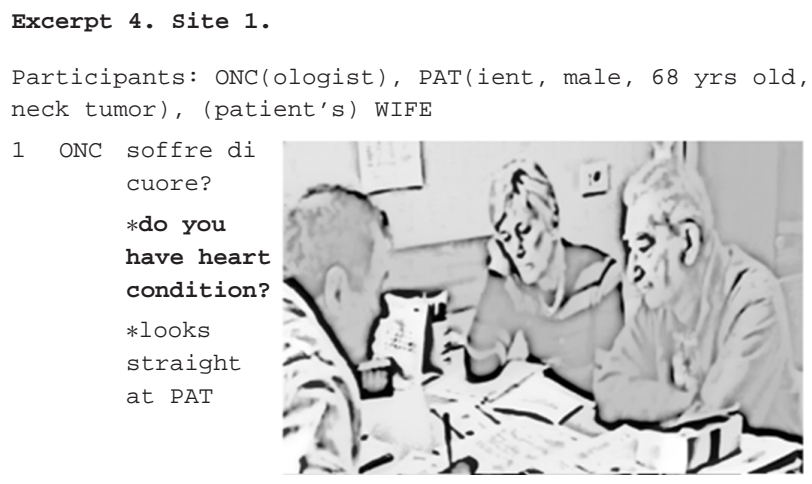

2 (1.0)
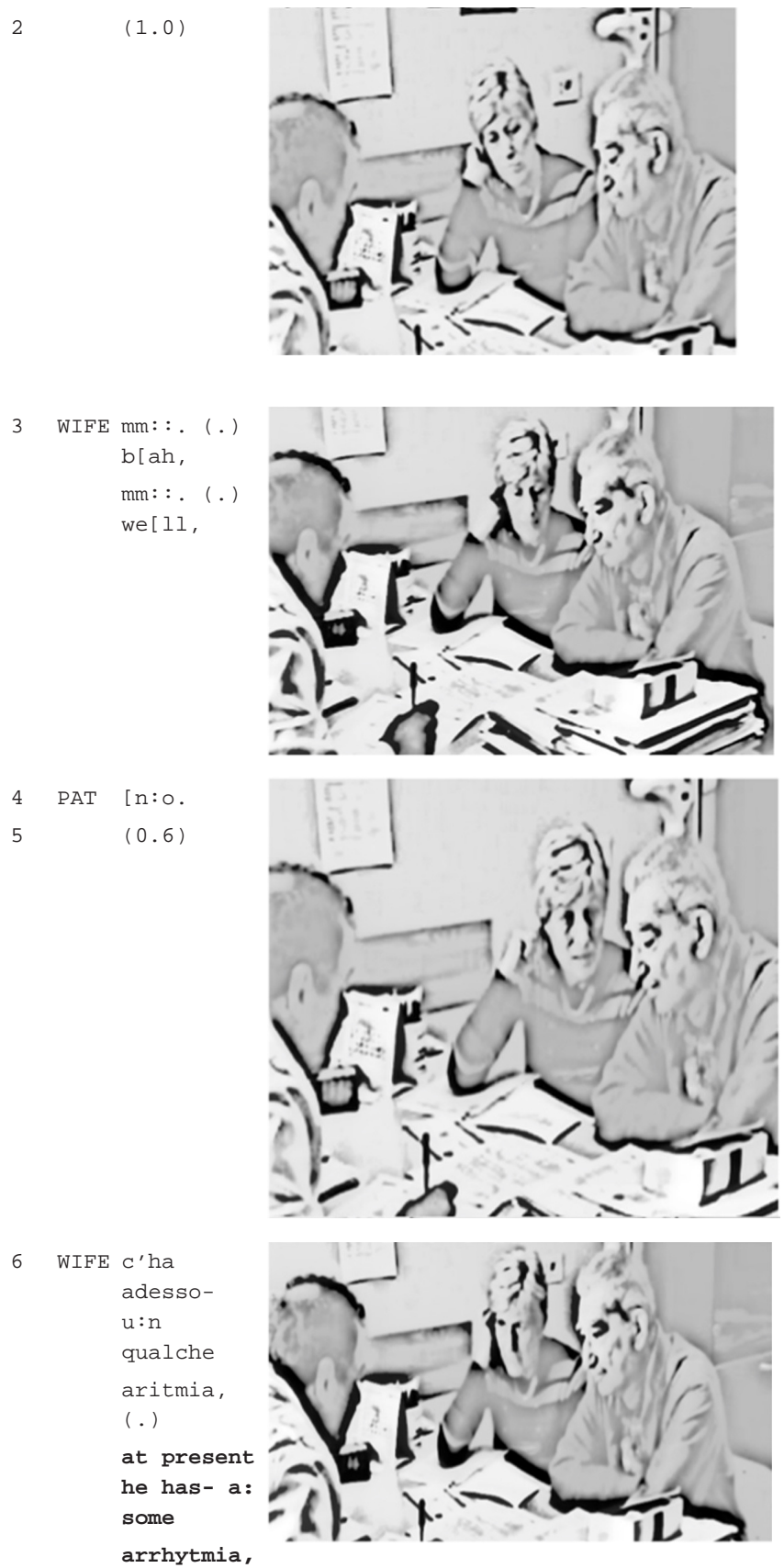

(.)

First of all, the doctor gazes at the patient when addressing the question to him. The patient is then considered as the most legitimate teller of his own experience and illness history. Be the one who is entitled to talk on behalf of the patient, it is the wife who starts responding. We see that from line 3, where the wife assumes a partial posture of what Schegloff calls "body torque" (Schegloff, 1998): this is a kind of postural configuration whose main capability is 'to display engagement with multiple course of action and interactional involvements, and differential ranking of those courses of action and involvement' (p. 536). As a matter of fact, in the course of her response, the wife is concurrently 
engaged in the interaction with the doctor and the one with her husband, whom she continuously addresses her gaze, as monitoring that he can check and validate the truthfulness of what she tells.

At line 3, the wife's hesitation (mm::.) and the interjection "well," conveys the sense of a problematic delivery of her response. As she starts vocalizing, the patient slightly bends toward his right (the wife's direction), exhibiting an almost imperceptibly shake of his head, indexing a negative response. He then utters "no" toward his wife, and then looks again at the oncologist. Also, the wife maintains a prolonged look on the patient. As a matter of fact, the wife finally revises the patient's response, by reporting that the patient has in fact "some" arrhythmia.

Yet, there has been a mutual monitoring within the intimate couple, and an orientation to check each other and build upon their mutual knowledge, in order to provide an exhaustive and valid response to the doctor.

What is interesting is that as the definitive response is produced, the wife and the patient co-orient toward the doctor, gazing simultaneously at him.

The response given ultimately to the doctor builds temporally and incrementally upon a mutual coordination of gaze and actions done by both the patient and the companion.

\section{Negotiating Entitlement to Tell in Cancer Problem Presentation}

Cancer problem presentation shares some features with the history taking stage, and related allocation of participants' status: the patient, in fact, is the one who is entitled to report about information $\mathrm{s} /$ he has derived from previous visits and from surgery. The main difference is that this stage entails careful examination of the patient's narrative which, in this specialty field, is particularly assisted by medical reports such as, ultrasound, magnetic resonance, surgical reports. These are mentioned and provided as reference throughout the whole narrative about the discovery and diagnosis of the illness.

Excerpt 5 below shows how the companion actively contributes to the accomplishment of the presentation of the cancer problem. Here, the patient's husband revises and validates information provided by the patient, contributing to a precise reconstruction of the tumor first detection.

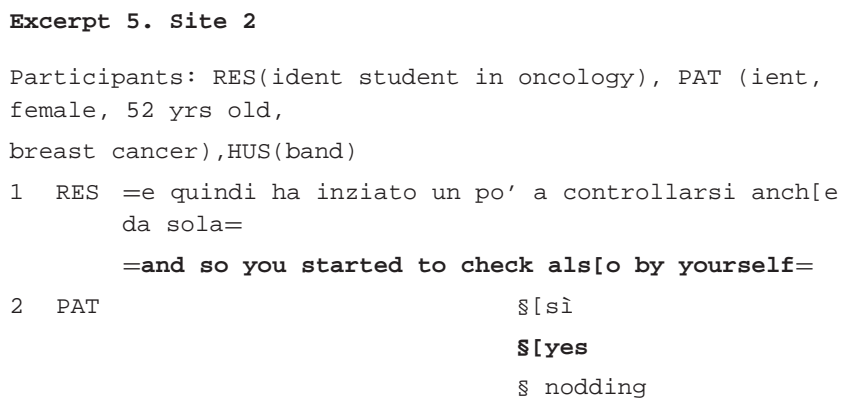

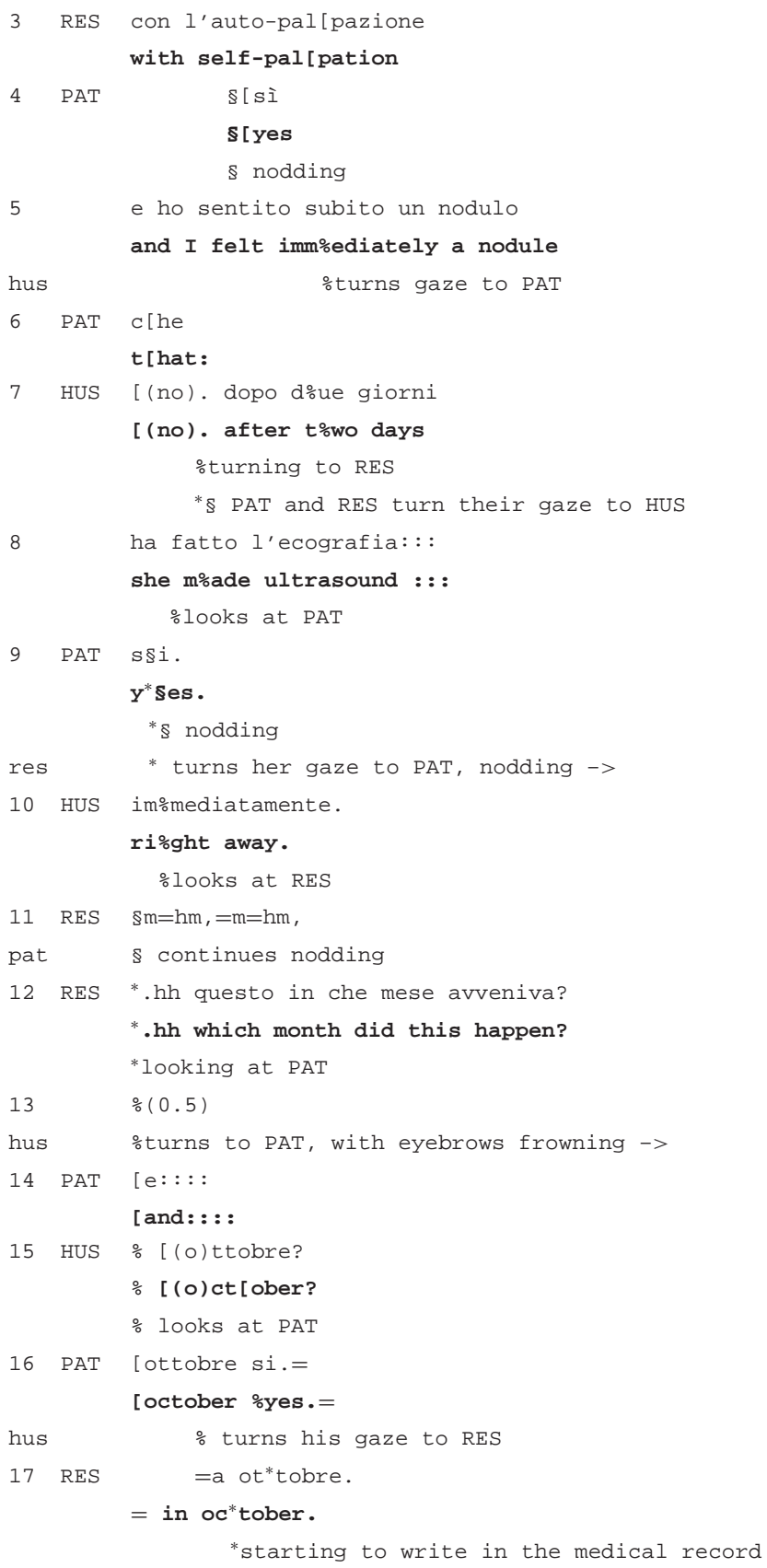

The patient's husband (who is gaze monitoring the patient during her report) enters the conversation in the midst of the patient's turn (line 6), revising her information about the timing of the realization of the tumor first discovered by (subjective) self-palpation and, later, by (objective) ultrasound. Despite he formally "interrupts" the patient, the husband's multimodal displays and particularly, gaze, work as signals that ensure that, throughout the course of all his turns, the patient's alignment and collaboration is constantly checked. Specifically at this regard, the husband turns gaze to the patient when he adds information about ultrasound. The gaze shift manifests the husband's sensitivity to the different knowledge statuses and entitlements of participants in this multiparty encounter 
(Goodwin, 1979). Throughout his turn addressed to the resident, the companion pursues the patient's validation of the information he is reporting, legitimating her as teller and experiencer of the story. Also, he mentions that the patient did ultrasound "immediately" (line 10): a reference that appears to account for a representation of his wife as a "moral" person who is able to understand the seriousness of a health condition and to engage in reasonable actions to take care of herself with no delay.

As a matter of fact, the resident continues to take the patient as her primary addressee (line 12). This does not discourage the husband to respond (line 15) and collaborate, this way, with the patient helping her to remember and provide accurate information. Yet, by addressing gaze to his wife rather than looking at the resident, he casts his contribution, rather than as a prompt for the patient, a check for validation from her, and he ascribes the wife as the one who has the ultimate right to provide a definitive response.

In his attempts to report the events in the right order and as much detailed as they happened, the companion shows to support the institutional aim of this stage, meaning, collecting information about the tumor as much reliably as possible. He does so, while also supporting the patient as a responsible agent in her illness story.

In the following excerpt, the resident is collecting the history of the illness: this routinely includes questioning the patient about when and how she discovered the cancer, how the diagnosis was made and other questions related to surgery, if the meeting is a post-surgical one. A quite high stake imposed over the patient during the problem presentation stage is that the patient needs to provide a reliable telling of the series of events and the evidences that $s /$ he collected prior to the meeting. Particularly when the companions are intimate partners of the patient, such as, spouses, who presumably shared the experience of their illness, they can entitle themselves to supply information asked to the patient, to add, elaborate, or revise them. The problem that surfaces in the example shown, is that the companion and the patient disagree over the timing of an event, which might prove important to tell the oncologist.

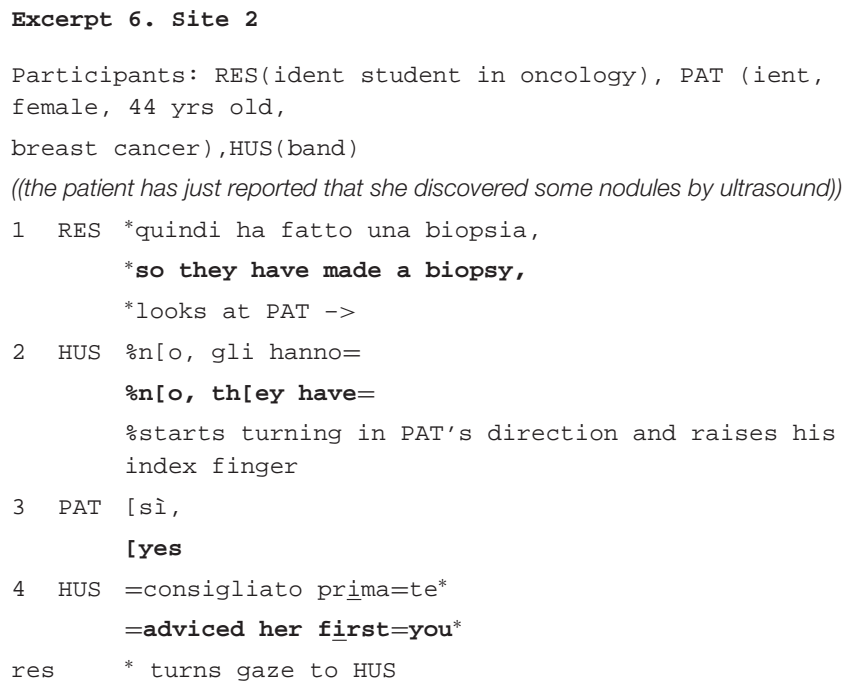

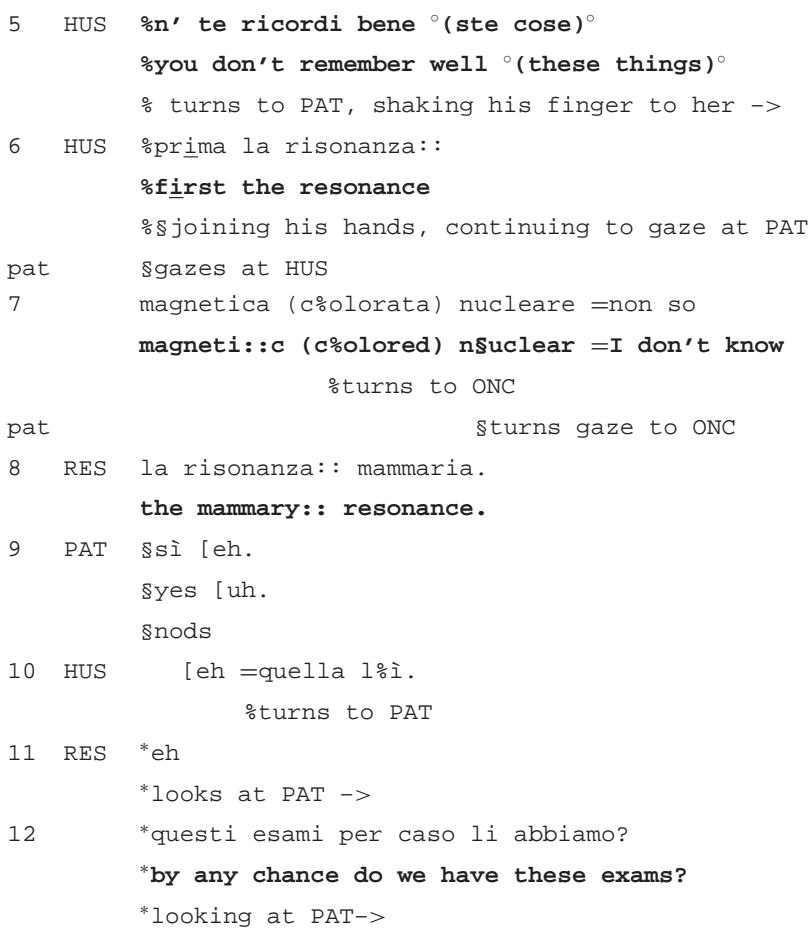

Despite the doctor's question was addressed to the patient, it is the husband who responds first (line 2). In partial overlap with the husband's turn, the patient provides her response (line 3), which confirms the resident's implication, and instead contradicts the husband. Prior to continuing in his telling, the husband turns to the patient and formulates to her that she does not remember well (line 5). What is of analytical interest is that the husband continues to look at the patient throughout his turn, and only turns to the doctor in correspondence with the word search for the exact name of the resonance (line 7). He also frames his turn with an evidential expression ("I don't know"), a stance marker by which he delivers to the doctor the ultimate responsibility and authority to tell about a medical matter.

The gaze shift to the doctor, together with the topical shift (from telling about when the magnetic resonance was exactly done to telling about the specific typology of the resonance) obtains that the husband subsides the disagreement he posed with his wife, with regard the temporal placement of the biopsy in relation to the magnetic resonance. From here on, the official interviewing between doctor and patient is restored: evidences of this are that the companion's response comes after the patient's one (line 10), that the resident only looks at the patient and she puts the sequence with the companion at a close, asking instead the patient about the availability of the documentation.

These analytical findings attest how the companion is capable to orient to the relevant aims proper of this stage, that is, providing information that is as much reliable, relevant and detailed as possible, to the doctor; however, while doing so, he also concurrently orients to not replacing or overshadowing the patient's voice. 


\section{"Sneaking" in Patient's Action During Cancer Diagnostic Assessment}

The next series of excerpts show instances where companions help the patient handling the documentation to the oncologist during the cancer diagnostic assessment stage. This stage has been reported a central stage of the whole visit. It entails the careful examination of, particularly, the histological test. This constitutes the bridging document to the recommendation for a treatment option and it implies that the oncologist engages in private diagnostic reasoning (Fatigante et al., 2020), particularly to assess the biological characteristics of the tumor and identify the most suitable treatment.

We have seen that companions closely monitor the patient's activity of handling the documents to the doctor, and sometimes their actions solely prevent a chaotic delivery of the documents.

The next example shows an extract from the consultation with a young patient, who came to the consultation with his wife and two other companions. He meets the doctor for a neck tumor, and he reported, during the history taking, that he was already treated some years before for an Erwing sarcome.

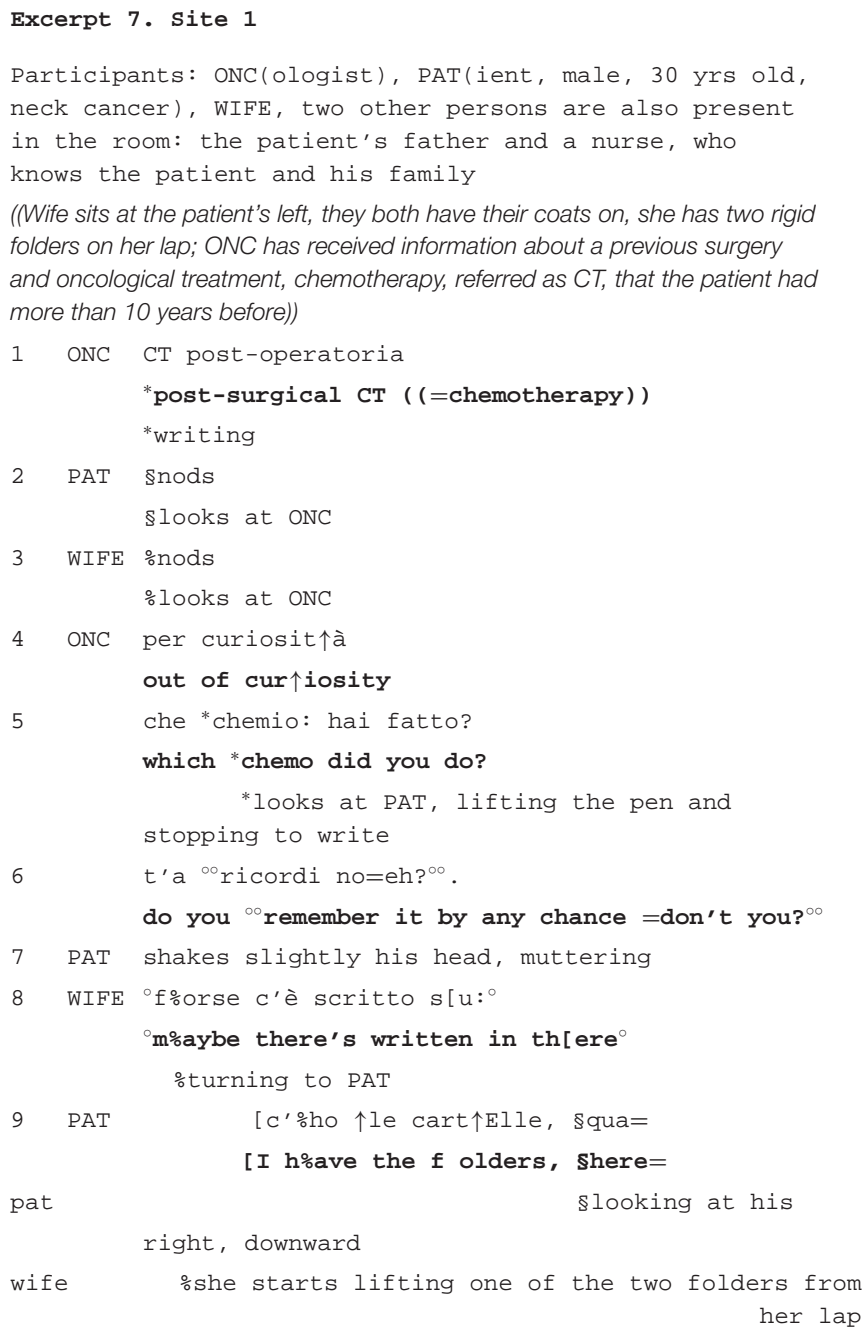

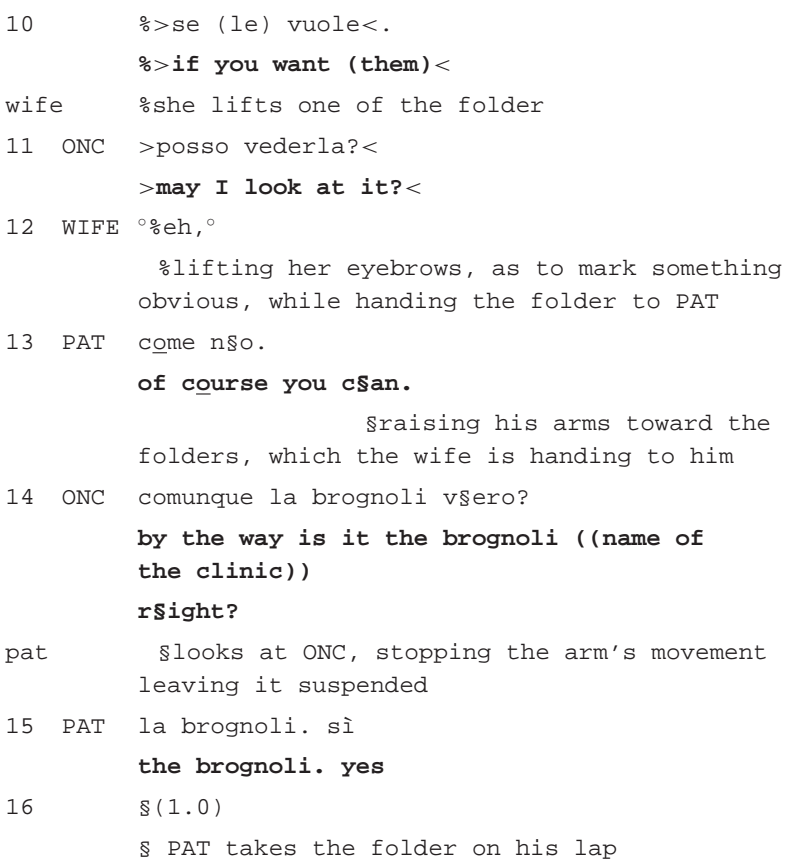

As we have observed in other visits of our corpus, the oncologist oralizes the information he writes (here, line 1), this way offering it at the scrutiny and confirmation of the patient (Sterponi et al., 2017; Fatigante et al., 2020). Following the oncologist's turn, the patient and companion nod concurrently, thus exhibiting themselves as having equal access to the information questioned and, further, to be equally legitimated to confirm it. In response to the oncologist's question whether the patient remembers the type of chemotherapy he did (several years before), the patient communicates that he does not remember. The patient's nonverbal token-the head shake (line 7)- undoubtedly conveys a clear reply, which would put to a close the inquiry. However, the patient's wife comes in the conversation (line 8) indicating the chance that what the doctor is looking for might be found in the written documents, contained in one (of the two) rigid, plastic folders which she carries on her lap.

Note that the companion whispers to the patient, and shows immediate proneness to hand the folders to him, but she never mobilizes (Stivers and Rossano, 2010) the patient's gaze to herself, nor she pursues in any way that he replies to her. That is, the companion's turn is crafted as an interstitial move and it is not expected by her, nor it is responded to by the patient, as a first part of an adjacency pair. Rather, it works as something that help the adjacency pair of question-answer between the doctor and the patient to continue smoothly. The patient, in turn, does not even wait for the end of her wife's turn to address a proposal to the doctor. The patient takes the wife's suggestion as a chance to provide the doctor with material evidences (which he refers as "his own," despite the fact that he did not mention them before nor he noticed them as currently useful) from which the doctor himself can draw the information. So doing, the interaction flow between the doctor and the patient is never halted. Both the patient and the wife contribute to this. Correspondingly to the 
patient's proposal at line 9, and before any solicitation from him, the wife lifts the folders from her lap and starts handing one of them to him. Her move also anticipates the explicit request by the doctor, which she seems to assess as somewhat expected (line 12). It is also noteworthy to observe that, as the oncologist recruits the patient again to verbal interaction, the patient pauses the action he was entertaining with his wife (bringing the documents that she is handling to him), and makes also the wife wait. In this sense, the companion's move adjusts and closely follows the flow of interaction between the patient and the doctor as it goes.

Summing up, the contribution of the patient's companion here was essential, given that she uniquely made material surroundings, which might go "unnoticed" to the patient, available to both him and, ultimately, to the oncologist.

On the other hand, her contribution did not disrupt in any way the patient's interaction with the doctor.

We show another example that displays, however in a different fashion, how the companion is concerned that the patient properly responds to the doctor's questions and expectations.

In the excerpt 7 , the patient (70 years old) is handling the relevant documentation regarding the surgery of the tumor to the doctor, and the doctor is transcribing the information in the medical record. The patient and her husband are monitoring the doctor's writing, looking at his pen on the sheet, remaining silent, while the doctor is oralizing (Sterponi et al., 2017). The doctor stops, quizzing about an incongruence in the chronological order of the documents.

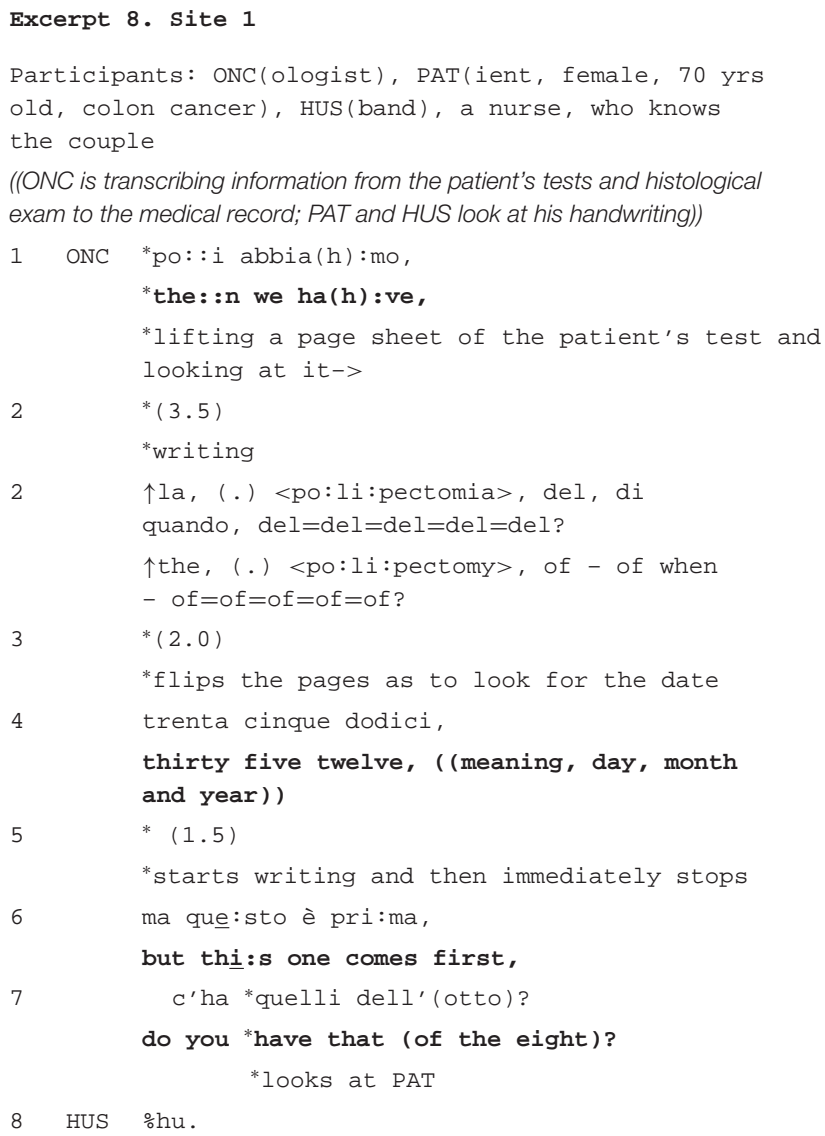

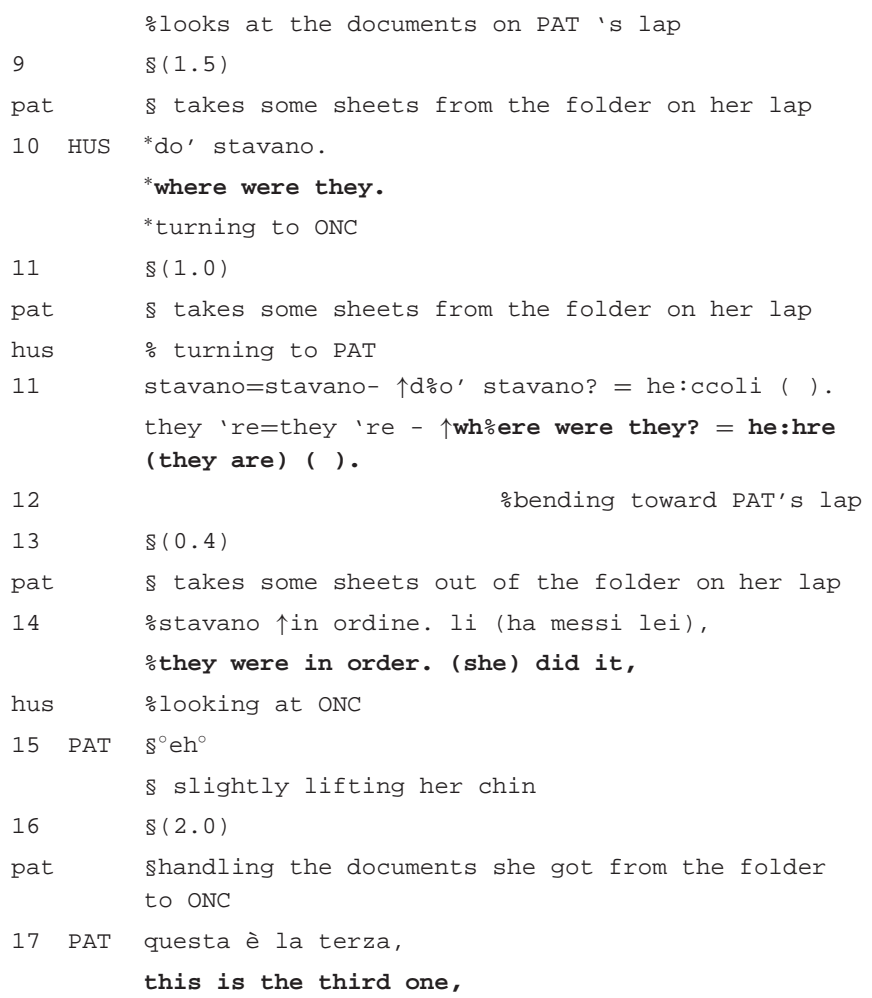

As we have already seen in the previous excerpt, the patient and her companion orient to the doctor's-sometimes longactivity of reading and transcribing remaining silent and gazefollowing the oncologist's gestures throughout the writing. When the oncologist raises the problem about the incongruent order of the documents, the companion follows: first, uttering a change of state token $(h u$, line 8$)$, i.e., an item that marks the acknowledgment of a certain piece of information as new and unexpected (Heritage, 1984b); then, he asks where the (missing) documents are.

In both ways, the patient's husband makes explicit his surprise with the current state of affairs, something, which makes him siding with the doctor's stance. Thorough his question, he looks in the direction of the patient's lap. So doing, it remains unclear whether he takes the wife as accountable for having messed up the documents or, more likely, he only utters the question as a generic marker of disappointment, which casts him as fully aware of the gap in expectations now having surfaced. His attempts would, then, work as to account for the poor performance that his wife and him are giving to the doctor. On the other hand, the wife does not visibly express such a preoccupation "about face" (Goffman, 1959) and provides no justification for the delay she eventually hands the document to the doctor (line 17). This and other similar instances show the companions' work in making relevant (either marking or, repairing) gaps in the patient's conduct, that is normatively expected in this context. It could also be that, being them more "free" from the pressure of both interacting with the doctor and being less emotionally loaded for the matters discussed, the companions can be more alert and they can also be more 
sensitive and available to exhibit a repair work of issues of face and performance.

\section{Brokering the Doctor's Explanations in Treatment Recommendation}

The treatment recommendation is a particularly crucial stage of the visit: it incorporates the main institutional objective of this medical encounter, i.e., delivering a treatment plan for the patient, and it implies relevant efforts at both informational and emotional level for both parties at interaction. ASCO (American Society of Clinical Oncology) guidelines recommend that doctors describe all treatment options available, telling the patient the benefits and burdens of treatment and enabling the patients to understand and weigh them in order to engage in decision making (Gilligan et al., 2017). At the same time, previous studies have demonstrated how doctors are aware of the fears that patients have with regards certain therapies (mainly, chemotherapy) (Mulders et al., 2008; Davies and Yeoh, 2012). This infuses the work of providing information with a commitment to reassure them and lessening these fears, in the service of providing the patients with the most promising option for them (Sterponi et al., 2017; Fatigante et al., 2020). Studies indicate that companions, particularly if family members, play a relevant role in this stage, getting involved in decision making (Albrecht et al., 2010).

During the treatment recommendation stage, the patient is mostly oriented to listening to the oncologist's explanations that account for the different treatment options. Frequent nodding, production of continuers, acknowledgment markers (e.g., sure, right...) are among her/his most frequent contributions. Companions perform a similar work, but also, sometimes engage in more extended turns, that elaborate the doctor's explanation and facilitate doctor and patient's understanding of the (complex) information discussed.

In example 8 , the doctor is anticipating to the patient the probable reason for why the surgeon (who has not yet operated the patient) has referred her to him. This implies the chance to reduce the size of the tumor by recommending the patient to undergo chemotherapy (so-called neo-adjuvant therapy) prior to the surgery. The explanation is not fully grasped by the patient, whereas, it is taken by the husband, who adds further material in order for the patient to understand.

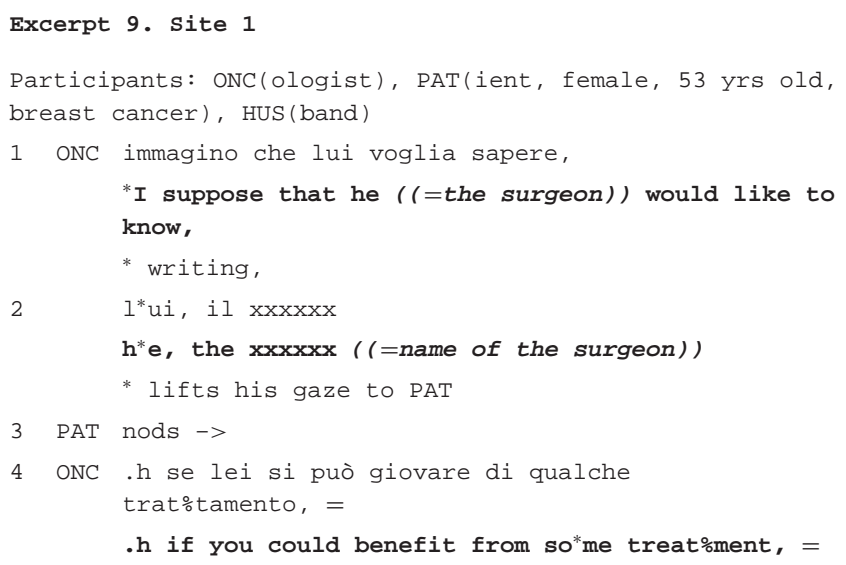

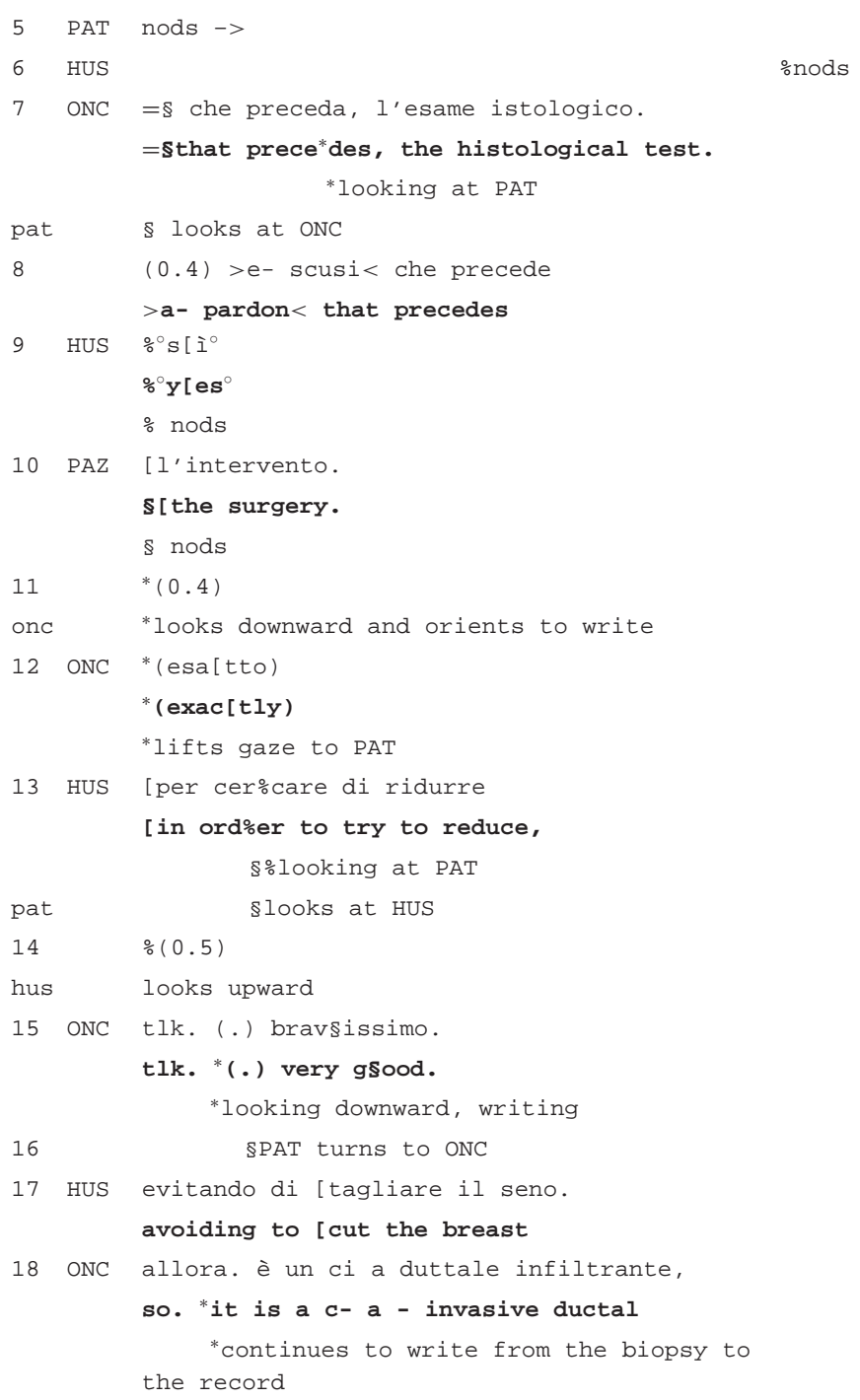

Until line 12, the companion and the patient are aligned as listeners of the doctor's turn, and they show to attend to the agenda of activities displayed by the doctor's both verbal instructions and physical actions, such as, writing. At line 13, the companion self-selects and adds original, medically relevant information, useful for brokering the patient's understanding with regards the benefit of undergoing a treatment before the surgery. This, together with the positive assessment he receives by the doctor, reveals that he has some knowledge regarding the conventional paths followed in the case of an oncological illness (he mentions, in another part of the visit, that his father had cancer and died for it). It is of interest for our discussion, that husband gazes at the patient at the beginning of his turn, addressing her as addressee of his talk. Immediately after, though (line 15), he looks upward, as if disengaging from this role of informant, thus rapidly clearing up the opportunity to engage in an open interaction with the patient, which would compete to the explanatory activity led by the doctor until now. 
The example, then, manifests another evidence on how the companion works in a strictly contingent and situated manner with the local ongoing activity. By making sure that the patient understands the rationale of the oncological actions, he maintains orientation to an interstitial participation in placing his formulation at the margin of the official interaction between the doctor and the patient.

Both verbal and multimodal aspects concurrent to the delivery of the companion's turns attempt to convey a "double affiliation:" respectively, with the doctor, who is still acknowledged the institutional authority, and with the patient, who is helped to understand the implication of the oncologist's talk.

Excerpt 10 also displays how the companion's (here again, represented by the husband) contribution, although minimal, may be heard as overtly affiliating with the oncologist, and supporting the specific activity, i.e., the delivery of a treatment recommendation, that is relevant at this moment in the visit. The excerpt is taken from data in Site 2; the resident has just delivered to the patient the proposal of chemotherapy as the most advantageous treatment for her (lines not reported), and summarizes here the reason that supports such a proposal.

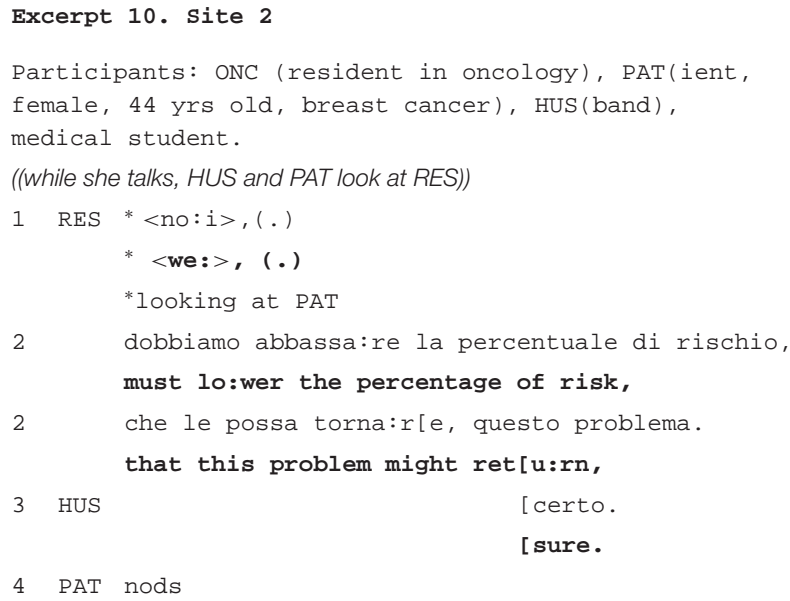

Both the patient and the companion display their availability as recipients of the doctor's explanation and recommendation, in that they maintain a sustained look to the resident throughout her formulation. Yet, the companion does more than that, producing a clear assessment ("sure," also meaning "that's right") of the oncologist's formulation (line 3). By making an assessment of the doctor's pronouncement, he signals that he embodies the oncologist's perspective (Goodwin and Goodwin, 1987) and supports the validity of the doctor's treatment recommendation. So doing, the husband's contribution obtains to sequentially prepare and shape the patient's orientation to eventually agree with the content of the formulation (line 4).

Summarizing, through many different cases and patients with different ages and cancer types, we have found that companions in this stage appear to support the importance for the patient to understand and accept the treatment proposed. Therefore, their contribution may be heard as essential for soliciting the patient to embrace a decision. At the same time, the in-depth analyses of how they intervene add to the main finding of the study, for which they tend to not "grab the spotlight" upon themselves and not interrupt the official interaction between the patient and the doctor.

\section{Introducing Practical Matters in the Outline of Future Actions Stage}

We discuss one example from the stage we labeled "Outline of future actions" that occurs after the main and most delicate business of this kind of encounter (the discussion of the treatment option) has been done. First, we have to highlight that, in this stage, the companions are consistently observed as being more active in recruiting the doctor as their own addressee, asking him questions about practical concerns related to the therapy such as, its duration and the ways in which it will be delivered. Questions about the side effects of the therapy are also voiced by companions more than by patients. ${ }^{1}$ A study by Eggly et al. (2006), which coded both the companions' and patients' questioning behavior, also found that the former more often raised issues about the management and logistics of treatment procedures. The companions' contribution appears as of utmost relevance, due to the fact that patients in first visits may be overwhelmed by the quantity and complexity of information they get in the previous stages, which they also need to process while they are presumably in a state of anxiety and concern (Annunziata and Muzzatti, 2012; Bronner et al., 2018). If we look at these instances, we find that the companion's question is taken by the doctor as a chance to deliver the information to the patient also. See at this regards example 11:

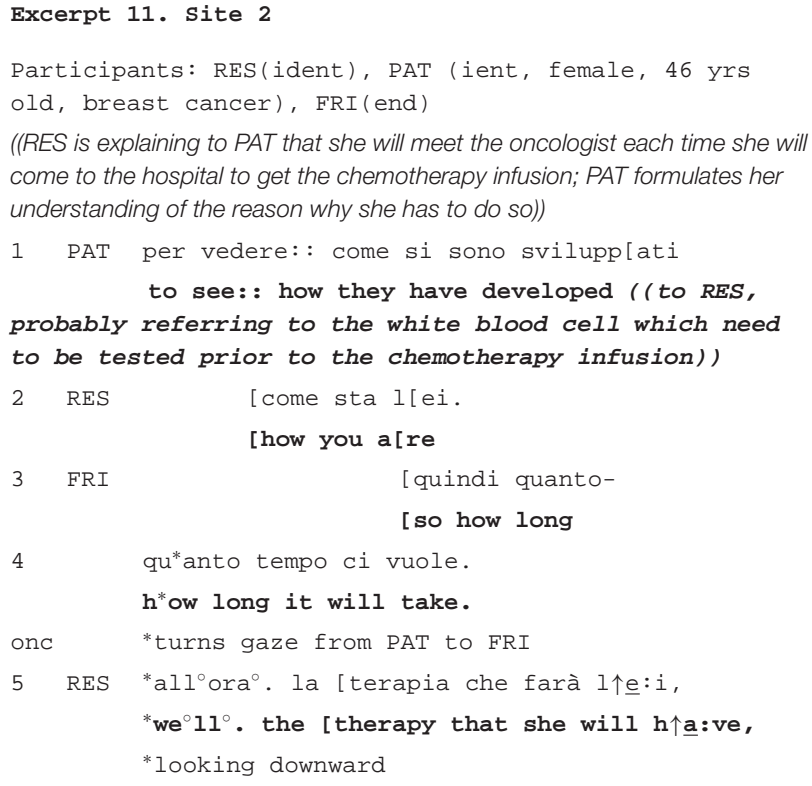

${ }^{1}$ On a subcorpus of 22 visits examined for other purposes, we have done a preliminary examination of the number of the companions' questions in the different stages of the visit, finding that the stage "Outline of future actions" contains the highest number of questions than in any other stage: 116 questions vs. 34 and 24 in, respectively, the stages of Treatment recommendation and the Cancer diagnostic assessment. 
6

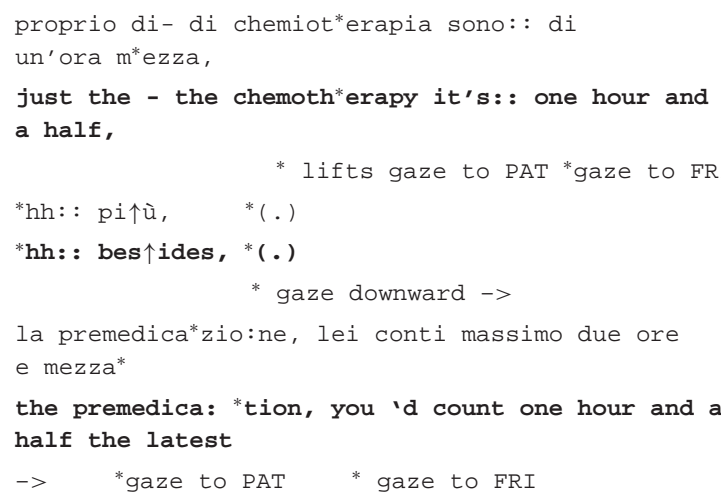

la premedica*zio:ne, lei conti massimo due ore e mezza*

the premedica: *tion, you 'd count one hour and a half the latest

-> *gaze to PAT * gaze to FRI

The companion here self-selects as the resident's previous turn approaches completion and she asks about the duration of each infusion. In responding to her, the resident removes her gaze and starts looking downward, as if planning her response. When she lifts her gaze, she looks at the patient and not at the companion, and she only shifts gaze to the companion when she tells the measure (one hour and a half, line 6 ), which was exactly what the companion asked. Further, as she expands her response, adding details, she explicitly selects the patient (line 8), turning again to the companion only at the end of the turn, and correspondingly to the mention, again, of the exact length.

The next and final example shows a similar case. Here, the patient has shown her distress in apprehending that she will get chemotherapy. The resident is adding information about the way she will get the chemotherapy infusion and she tells the patient that she will be implanted a device, the portacath (Port), which will give access to veins for regular administration of the drug.

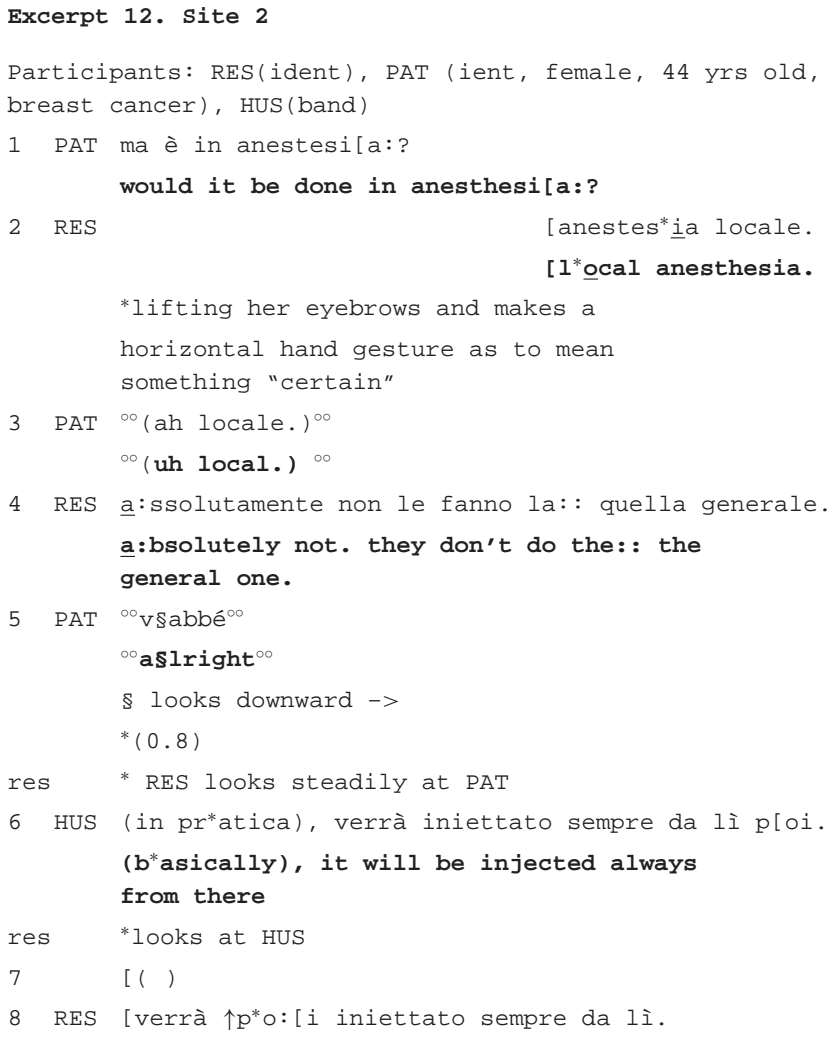

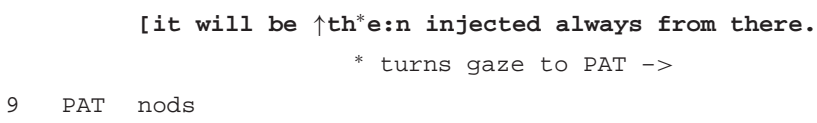

Here again, the companion self-selects to ask a question to the resident. Though, early in the construction of her response, the resident shifts gaze to the patient (line 8 ) and never returns it to the companion, although the topic is maintained for few lines more (lines not reported). This and other examples make clear that, even in cases where the companions clearly constrain doctors to attend to them as primary interlocutors (as in question-answer pairs), the sequential and multimodal construction of the doctor's answer is crafted in such a way, as to maintain companions, and not the patients, as "audience" to the doctor's response.

\section{"Speaking As" the Patient: An Example From the Physical Examination}

Among our excerpts, we found one single instance in which the companion casts the patient in the third person, apparently speaking on her behalf.

We first provide the context of the sequence. The exchange takes place as the visit approaches conclusion (minute $33^{\prime} 47^{\prime \prime}$, where the overall length of the visit is $40^{\prime} 22^{\prime \prime}$ ). In this visit, the patient meets this oncologist for the first time. However, she had already experienced cancer long before. Prior to this sequence, the patient has made repeated attempts to be reassured by the oncologist, regarding a specific concern, i.e., her fear that a certain value, called CA 125 (amount of the protein cancer antigen 125 in the blood), can grow: CA 125 is sometimes used as a marker of cancer recurrence in patients with ovarian cancer, like the patient in this visit. The doctor has engaged in a long explanation about this, where he told the patient that an increase of CA 125 is also observable in a variety of other, non-oncological and non-risky conditions. For this reason, he recommended the patient to stop persisting in what he called an "obsessive" measuring of the marker (the patient also described herself as "crazy" about checking the marker), ironically stating that "otherwise she would spend all her life to check the CA 125." At this point, the doctor invites the patient to undress in order to proceed to the physical examination. Throughout the doctor's explanation that has occurred up to now, which has also included that the doctor has gazed to both the patient and the companion, the companion has remained silent. He only takes turn as the doctor asks the patient to proceed to the physical examination:

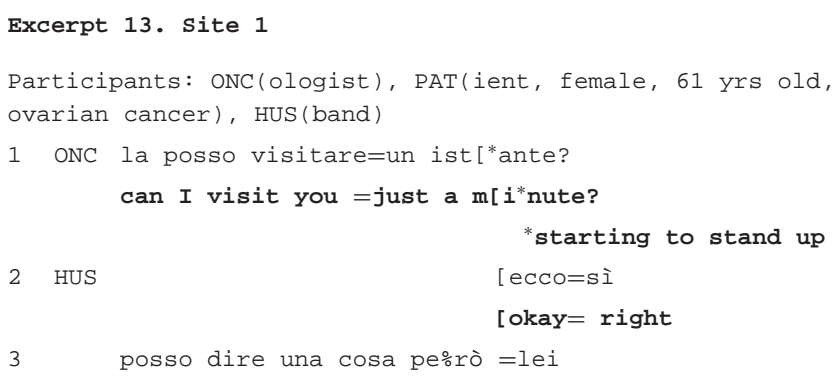


may I say something thoough =she

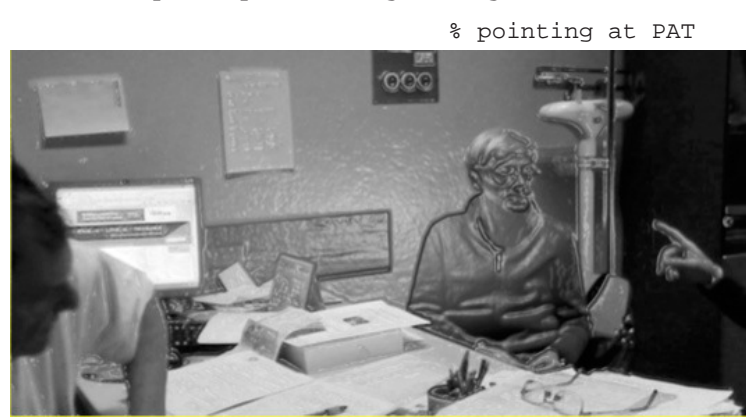

4 ONC eh.

5 HUS però:, dicia:mo

$$
\text { ye: } t \text { I mea:n }
$$

6 I\$e:i, (0.5), a parte che lei dice. so' un po' matta.

s\$he:, let alone the fact that she tells. I'm a little crazy

pat sgets up to undress herself

7 però, sa che cos'è?

yet, you know what?

8 ONC eh. ((standing, out of the camera))

9 HUS in tultti questi anni, dall'ottantaquattro a oggi, =

in all these years, from eighty four till today, = ( (meaning, from

year 1984))

$10=$ si è sẹpre verificato, che:, it has a:lways happened, tha: $t$,

11 lei è particolarmente sensibile, a questo CA centoventicinque.

she is particularly sensitive, to this CA one hundred twentyfive.

$12 \quad(1.0)$

13 ecco >quindi lei si è fatta< una sua teoria

here it is. > therefore she built $<$ her own theory

14 PAT la $x x x x$ ((=name of the radiologist)) (mi rende sensibile).

the $\operatorname{xxxx}((=$ name of the radiologist)) (makes me sensitive).

15 HuS che poi d'altronde, (.) io che le sto vi[ci:no, on the other hand, (.) being close to her,

16 PAT [no ma anche la xxxx mi ha detto,

[no but also the $\mathbf{x x x x -}$

((=name of the radiologist)) told me,

17 HUS noi non siamo- per niente medici >quindi è chiaro:<,

we are absolutely not- doctors $>$ so

it's o:bvious $<$,

18 PAT no ma anche la $\operatorname{xxxxx}$ ((=name of the radiologist)) mi ha detto,

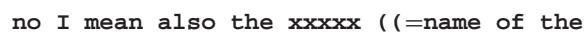

radiologist)) told me,

( (in the lines following, the patient recycles her argument))
We need first recall that the oncologist has already read the series of medical diagnostic documents that the patient has brought to the visit, and formulated his opinion to the patient. Therefore, we can consider that the stages of Cancer Diagnostic Assessment and Treatment Recommendation (here, involving an advice for a "wait -and -see" strategy) have already occurred. The companion's initiative at line 2 is perfectly timed: he enters in correspondence to the TRP (Transitional Relevance Point; Sacks et al., 1974) but also at the juncture point of the activity transition, marked by the oncologist's question and his postural change (he stands up).

At the same time, his intervention poses a problem, in that he recruits the oncologist's attention, in a moment where the oncologist is just leaving from the attentional visual field (the desk) available to interact face to face with the husband. In this sense, the companion's move could be sensed as inappropriate and untimely. The companion anticipates the unwelcomed consequences of his possibly inappropriate move by a presequence (line 1, see Schegloff, 1980, 2007), a polite formulation which works in order to obtain a go-ahead response from the doctor, prior to the telling; by doing so, the companion also shows his reflexive awareness that he might be not fully entitled, in this context, to address original concerns. Last but not the least aspect we want to emphasize, the husband points at his wife with his finger, this way maintaining her-who was also just about to stand up- as involved in the telling. His pointing action solicits, in turn, that the wife turns gaze at him and awaits (until line 6, when she stands up). In the turns that follow (lines 7-13), the husband advocates the patient's reasons to voice her concerns to the doctor, regarding the CA125 marker. Noteworthy, the companion does not support the patient's concern that the marker can indicate a progression of the illness. This argument has been clearly rejected by the scientific explanation that the doctor has provided to her in the lines prior to this sequence (not reported). Rather, the husband supports the patient's entitlement to develop her own theory, due to the evidences that she gathered through time (line 8), on a period, upon which she can uniquely claim experience. In so doing, the husband engages in repair work as regards the potential face loss (Goffman, 1959) that the wife had experienced in the discussion with the doctor; he does so, by strengthening the epistemic bases of her claims which, although typologically different from that of the doctors (see the disclaimer at line 17, "we are not doctors"), are notwithstanding viable to shape a personal explanatory theory of the problem. Note that the husband juggles from emphasizing the wife's oddness (line 6), this way apparently siding with the doctor against her argument, to validating the wife's evidences, by putting them in historical context. In these terms, what the husband does could be righteously captured by what Mazer et al. (2014) call as "speaking about" the patient. Accordingly to that categorization, here the husband's "role" would be that of an external observer, who infers "the patient's cognitive processes" (she built her own theory, line 13) from an external and independent assessment. How such a categorization is vulnerable to instability is, though, clear as we come to line 15 immediately following, when the husband claims to be "close to her" (line 15), and thus invokes 
his own position as co-experiencer of the patient (Mazer et al., 2014).

However, the husband maintains a certain ambiguity (also surfacing in his vague formulations, as in "she is particularly sensitive" or in the elliptical sentences at line 15) as to whether he is embracing the patient's view or just attempting to repair her face loss, his intervention helps indeed the patient to recycle her argument: that is, the patient takes advantage of the companion's turn, to invoke the radiologist's opinion (lines $14,16,18$ ) and build a new confrontational arena for the oncologist to respond again to her issue.

That the oncologist opinion here is called into question again is also demonstrated by the husband's disclaimer "we are not doctors" (line 17), that mitigates (Caffi, 1999) the validity of the propositional content alluded by his intervention.

With this final example, we showed how, during the first oncological visit, taking over the patient's position and speaking for or about her is a complex endeavor for the companion. Particularly, and whether or not it is intended to support or diminish the patient's agency, the companion's move displays as never independent from the sequential context and the existing constraints of the participant framework that are active in the visit at any specific moment. We add, as a final point, that the companion takes active initiative as the main verbal flow of interaction the patient and the doctor is momentarily suspended, in favor of an activity which, involving the body inspection of the patient, is managed as separate and self-bounded. This adds as an ultimate evidence of the companions' attention to act at the margins of the main, official discourse activities or at most, at the interstices between activities.

\section{DISCUSSION}

In this paper, we conducted a close analysis of how the companions of cancer patients who are present at the first oncological visit contributed to the accomplishment of the visit and its different aims. Our results add and complement those, reported from previous studies on the topic, which indicate that, overall, companions present in the visit facilitate the communication between the doctor and the patient (LaidsaarPowell et al., 2016). Our analyses confirm this result in the specific oncological setting examined, and they show how the companions facilitate doctor-patient communication in rather specialized ways, i.e., by supporting the specific institutional aims and activities related to each different stage of the visit.

Summarizing, and precisely focusing on the temporal and sequential arrangement of the verbal and multimodal resources that are employed by all participants in the visit, our analyses uncover some regular features.

Firstly, we showed that the companions design their turns as to acknowledge and preserve the doctor- patient talk as the main and official course of interaction since the very beginning (excerpts 1-2). This is primarily revealed by instances, where the companions deliver their turns-however sometimes essential to the accomplishment of specific activities (excerpts 3, 4, and 7)-in a reduced volume or, where they "mask" or lessen the visibility of their actions in such a way, as to not recruit the participants' overt attention. Companions also allowed themselves to openly enter the conversational floor and publicly recruit all other co-participants' attention, but this only happened at certain moments in the visit, that clearly come after the topical business of the visit (i.e., diagnostic explanations and treatment recommendation) has been discussed (excerpts 11-13).

This maintains the patient as the most responsible and legitimate agent in the interaction and allows that the patient's interaction with the oncologist runs smoothly. The examination of multimodal resources was particularly beneficial to the investigation of this preference. We, thus, discovered that, when the companions speak on behalf of the patient, they finely negotiate with the patient, by means of constant gaze monitoring and gaze shifts, their own entitlement to speak (excerpts 5-6). We also noted that doctors responded to companions' either verbal and non-verbal turns by "rushing through" the interaction with them, and returning rapidly to the patients, as when they reply to the companion's question addressing the patient or the companion-patient pair together (excerpts 3,6). By doing this, they honor the patient's status as their proper interlocutor, at all stages of the interaction and informational flow.

A second strong finding is that companions show to attend to the particular goal orientation (Drew and Heritage, 1992) of each stage of the visit. Gaze shifts, gestures, modulation of the tone of voice (e.g., whispering), pausing, interstitial placement of actions such as, passing documents silently, or muttering, help companions to attune to the specific pragmatic constraints regulating the transitions between stages and activities (Mondada, 2006; Deppermann et al., 2010). By including all these multimodal resources, the analyses pointed out how the companions revised and strengthened the patient's report during the Anamnesis and Problem presentation (excerpts $3-6)$; they helped in the provision of documents to the doctor and monitored carefully both the patient and the doctor's conduct during the screening of the patient's records during the Cancer Assessment stage (excerpts 7-8); they supported the oncologist's formulation and bridged doctor-patient mutual understanding in stages, where the doctor delivered explanations and instructions during the Treatment Recommendation stage (excerpts 9-10); they postponed their more active interventions (e.g, questions) to the end of the visit, orienting to the institutional goal of warranting the patient the more information as possible about how to practically manage the therapy once the visit is over (excerpts 11-12).

Thirdly, contrary to what identified by previous studies on the topic (cf. Street and Gordon, 2008; Jansen et al., 2010), our research does not report enough evidences of the companion's provision of "emotional support" to the cancer patient. That is, we did not observe explicit attempts by the companions to provide reassurance or comfort to the patient, nor instances in which they explicitly check with the patient, or voice to the doctor, the patient's feelings. We may not exclude that companions in our corpus did so, later and outside the consultation room; yet, it appears that, throughout the visit, companions prioritize those contributions, which are beneficial (essential in some cases) to the accomplishment of the instrumental tasks embedded in the first oncological visit, rather than to soothe, or formulate, the patients' possible discomfort 
or anxiety. On the other hand, we also take a cautious stance with regards the term emotional, in that, as Ruusuvuori (2013: 230) maintains, identifying how-and by whom-emotions are dealt or expressed in talk is a challenging task. Keeping close to the evidential ground of our transcripts, we have seen that the companions displayed affiliation toward the doctor (excerpt 1 line 7, excerpt 3 line 17) and sometimes appeared to be openly judgmental of the patient's performance (excerpt 6 line 5, excerpt 13 line 6); yet, when this happened, they managed, via gaze and sequential placement of their turns (such as, withdrawing and waiting that the patient takes her turn or validate their responses, removing gaze from the participants' and leaving that the doctorpatient interaction is resumed, attempting to get the patient involved again etc.) to restore and balance patient's entitlement to participate, thus affiliating to her/his status as primary teller of her/his experience. Excerpt 13, in particular, showed a case in which the companion does "repair work" (Goffman, 1959) toward the patient's face, by supporting her entitlement to have a say upon her own experience. Despite the husband points to the patient's "sensitivity," it is not emotions that are made relevant but, rather, the patient's opportunity (which she takes advantage of) to recycle her argument and ground it onto more authoritative bases (i.e., the support from another doctor).

That is, however the husband's move might be intended to assist the patient in her emotions, the development of the sequence showed how it encountered a different treatment and interpretation. It may be that these results are constrained by the particular institutional and interactional context of this kind of visit: this is an event, occurring between people who are mutually strangers, primarily designed to deliver information and instructions for the patient about what treatment to begin and how to perform that treatment (Sterponi et al., 2017; Fatigante et al., 2020). Emphasis is placed upon the need that the patient understands-and agrees with-the epistemic bases (i.e., the explanation of benefits and risks), which favor certain treatment options instead of others (see also Costello and Roberts, 2001; Collins et al., 2005; Gill, 2019; Tate and Rimel, 2020). The discussion of psychosocial concerns-which may also threaten the patient's availability to agree with the proposalis at most postponed to the accomplishment of the main task of the visit, and they are certainly not dedicated a proper and specific stage.

A final point we discuss in relation to the reviewed literature, is that our study does not support findings, which indicate that the presence of the companion can generate tensions with the patient, due to marginalization or censorship of the patient's voice (cf. Mazer et al., 2014). It might be that such tensions arise more in contexts, where the patient is elderly or more vulnerable than the patients in our corpus. However, we also add that we posed a particular attention to avoid assigning a unilateral judgment over the companions who took turns on behalf of the patient. As shown, the in-depth examination of the sequential multi-party arrangements of participants' turns demonstrate that the companions' actions are in fact always the result of a negotiation with the patients, who are not passive even when they remain silent (Heath, 1986). Furthermore, the doctors in our corpus ultimately appeared to refer to patients, or, at most to the companion-patient dyad as their ultimate addressee.

We conclude, by underlining that our study provide nuances to the role categorizations ascribed to companions by coding schema in previous studies (Ellingson, 2002; Street and Gordon, 2008; Del Piccolo et al., 2014; Mazer et al., 2014). In contrast to the emphasis on verbal conduct as primary means of interaction, and the focus on individual behavior implied by coding schema, the analytical procedure we have chosen closely followed the participants' actions in the local time and place they are produced (the conversational sequence). Within this perspective, we showed that the companions' roles, rather stable positions enacted by the individual actor, are instead highly mobile; they are tied to the specific contingencies occurring within the visit stages and activities, and they are the contextualized results of complex temporal, sequential, multimodal and multiparty arrangements of all participants' actions (these including minimal visual tokens such as eye movements and head/body inclines, or aural tokens such as sighs and hummings, rarely examined by coding systems).

The evidence for which the companions are able, by even small and interstitial actions, to support the accomplishment of the oncological visit's institutional aims and activities, emphasizes the potential relevance of their contribution in affecting both the visit's outcomes and the participants' satisfaction of their communication.

Our findings suggests the need to broaden the concept of the doctor-patient alliance into that of a doctor-patientcompanion alliance. Medical education, and interventions to implement patient's and companion/caregiver's literacy in oncology, have to consider the multiparty nature of the oncological communication. On the other hand, we demonstrated that the richness of doctor-patient-companion communication can be utmost revealed by analytic tools, such as multimodal video analysis of conversations, that shows carefully and preserves the local configuration of actions assembled moment by moment by participants (Mondada, 2006).

\section{DATA AVAILABILITY STATEMENT}

The data analyzed in this study is subject to the following licenses/restrictions: the data that support the findings of this study are not publicly available due to privacy restrictions. Requests can be addressed to the authors, as to only share transcripts of the interactions (with anonymized references). Requests to access these datasets should be directed to Cristina Zucchermaglio, cristina.zucchermaglio@uniroma1.it.

\section{ETHICS STATEMENT}

The studies involving human participants were reviewed and approved by Ethical Committee of Policlinico Umberto I, Ethical Committee of Department of Social and Developmental Psychology, Sapienza University of Rome and ASL (Local Health System) RM A, Rome. The patients/participants provided their written informed consent to participate in this study. Written 
informed consent was obtained from the individual(s) for the publication of any potentially identifiable images or data included in this article.

\section{AUTHOR CONTRIBUTIONS}

MF worked at the first draft of the results, which were revised by CZ first and FA. FA contributed to the writing of the introduction. $\mathrm{CZ}$ contributed to the writing of the Discussion and Implications.

\section{REFERENCES}

Albrecht, T. L., Eggly, S. S., and Ruckdeschel, J. C. (2010). "Communicating with relatives/companions about cancer care," in Handbook of Communication in Oncology and Palliative Care, eds D. W. Kissane, B. D. Bultz, P. N. Butow, and I. G. Finlay (Oxford: Oxford University Press), 157-164.

Annunziata, M. A., and Muzzatti, B. (2012). "Psychological distress in oncology," in Psychological Distress. Symptoms, Causes and Coping, eds M. A. Annunziata and B. Muzzatti (Hauppauge, NY: Novapublisher), 27-48.

Bensing, J. M., and Dronkers, J. (1992). Instrumental and affective aspects of physician behavior. Med. Care 30, 283-298. doi: 10.1097/00005650-199204000-00001

Bronner, M. B., Nguyen, M. H., Smets, E. M. A., van de Ven, A. W. H., and van Weert, J. C. M. (2018). Anxiety during cancer diagnosis: examining the influence of monitoring coping style and treatment plan. Psychooncology 27, 661-667. doi: 10.1002/pon. 4560

Caffi, C. (1999). On mitigation. J. Pragmatics 31, 881-909. doi: 10.1016/S0378-2166(98)00098-8

Clayman, M. L., Roter, D., Wissow, L. S., and Bandeen-Roche, K. (2005). Autonomy-related behaviors companion communication and roles of companions in Japanese geriatric encounters. Soc. Sci. Med. 60, 2307-2320. doi: 10.1016/j.socscimed.2004.08.071

Collins, S., Drew, P., Watt, I., and Entwistle, V. (2005). 'Unilateral'and 'bilateral'practitioner approaches in decision-making about treatment. Soc. Sci. Med. 61, 2611-2627. doi: 10.1016/j.socscimed.2005.04.047

Costello, B. A., and Roberts, F. (2001). Medical recommendations as joint social practice. Health Commun. 13, 241-260. doi: 10.1207/S15327027HC1303_2

Coupland, J. (ed) (2000). Small Talk. Harlow: Pearson Education Limited.

Craven, A., and Potter, J. (2010). Directives. Contingency and entitlement in action. Discourse Stud. 12, 1-24. doi: 10.1177/1461445610370126

Davies, E., and Yeoh, K. W. (2012). Internet chemotherapy information: impact on patients and health professionals. Brotish J. Cancer 10, 651-657. doi: 10.1038/bjc.2011.601

Davis, T. C., Williams, M. V., Marin, E., Parker, R. M., and Glass, J. (2002). Health literacy and cancer communication. CA Cancer J. Clin. 52, 134-149. doi: $10.3322 /$ canjclin.52.3.134

Del Piccolo, L., Goss, C., Bottacini, A., Rigoni, V., Mazzi, M. A., Deledda, G., et al. (2014). Asking questions during breast cancer consultations: does being alone or being accompanied make a difference? Euro. J. Oncol. Nurs. 18, 299-304. doi: 10.1016/j.ejon.2014.02.001

Deppermann, A., Schmitt, R., and Mondada, L. (2010). Agenda and emergence: contingent and planned activities in a meeting. J. Pragmatics 42, 1700-1718. doi: 10.1016/j.pragma.2009.10.006

Drew, P., and Heritage, J. (1992). "Analyzing talk at work: an introduction," in Talk at Work: Interaction in Institutional Settings, eds P. Drew and J. Heritage (Cambridge: Cambridge University Press), 3-65.

Duranti, A. (1997). Universal and culture-specific properties of greetings. J. Linguistic Anthropol. 7, 63-97. doi: 10.1525/jlin.1997.7.1.63

Eggly, S., Harper, F., Penner, L., Gleason, M., Moore, T., and Albrecht, T. (2011). Variation in question asking during cancer clinical interactions: a potential source of disparities in access to information. Patient Educ. Counsel. 82, 63-68. doi: 10.1016/j.pec.2010.04.008

Eggly, S., Penner, L., Hagiwara, N., Gonzalez, R., Harper, F., Heath, E., et al. (2013). Patient, companion, and oncologist agreement regarding information
All authors contributed to the data collection, the concept and design of the study, the planning of the contribution in its aims and methodology, and contributed to the collection and discussion of the analyses.

\section{FUNDING}

This work was supported by Sapienza University (Grant Numbers RM11916B7AD32B81 and C26A13EW2Y).

discussed during triadic oncology clinical interactions. Psycho Oncol. 22, 637-645. doi: 10.1002/pon.3045

Eggly, S., Penner, L. A., Greene, M., Harper, F. W., Ruckdeschel, J. C., and Albrecht, T. L. (2006). Information seeking during "bad news" oncology interactions: question asking by patients and their companions. Soc. Sci. Med. 63, 2974-2985. doi: 10.1016/j.socscimed.2006.07.012

Eide, H., Gravgaard, P., Holgersen, K., and Finset, A. (2003). Physician communication in different phases of a consultation at an oncology outpatient clinic related to patient satisfaction. Patient Educ. Counsel. 51, 259-266. doi: 10.1016/S0738-3991(02)00225-2

Ellingson, L. L. (2002). The roles of companions in geriatric patientinterdisciplinary oncology team interactions. J. Aging Stud. 16, 361-382. doi: 10.1016/S0890-4065(02)00071-3

Emanuel, E. J., and Emanuel, L. L. (1992). Four models of the physicianpatient relationship. JAMA. 267, 2221-2226. doi: 10.1001/jama.267.1 6.2221

Epstein, R., and Street, R. L. (2007). Patient-Centered Communication in Cancer Care: Promoting Healing and Reducing Suffering. Bethesda, MD: National Cancer Institute; U. S. Department of Health and Human Services; National Institutes of Health. doi: 10.1037/e481972008-001

Fatigante, M., Heritage, J., Alby, F., and Zucchermaglio, C., (2020). Presenting treatment options in breast cancer consultations: advice and consent in Italian medical care. Soc. Sci. Med. doi: 10.1016/j.socscimed.2020.1 13175

Fatigante, M., Zucchermaglio, C., Alby, F., and Nutricato, M. (2021). The structure of the first oncological visit: a conversation analytic study. Psicol. della Salute 1 , $53-77$.

Gill, V. (2019). 'Breast cancer won't kill ya in the breast': broaching a rationale for chemotherapy during the surgical consultation for early-stage breast cancer. Patient Educ. Counsel. 102, 207-215. doi: 10.1016/j.pec.2018. 09.002

Gilligan, T., Coyle, N., Frankel, R. M., Berry, D. L., Bohlke, K., Epstein, R. M., et al. (2017). Patient-clinician communication: American society of clinical oncology consensus guidelines. J. Clin. Oncol. 35, 3618-3632. doi: 10.1200/JCO.2017.75.2311

Glenn, P. (2003). Laughter in Interaction. Cambridge: Cambridge University Press.

Goffman, E. (1959). The Presentation of Self in Everyday Life. New York, NY: Doubleday.

Goodwin, C. (1979). "The interactive construction of a sentence in natural conversation," in Everyday Language: Studies in Ethnomethodology, ed G. Psathas (New York, NY: Irvington Publishers), 97-121.

Goodwin, C. (1984). "Notes on story structure and the organization of participation," in Structures of Social Action, eds M. Atkinson and J. Heritage (Cambridge: Cambridge University Press), 225-246.

Goodwin, C. (1987). Forgetfulness as an interactive resource. Soc. Psychol. Q. 50, 115-130. doi: $10.2307 / 2786746$

Goodwin, C. (2000). Action and embodiment within situated human interaction. J. Pragmatics 32, 1489-1522. doi: 10.1016/S0378-2166(99)00096-X

Goodwin, C., and Goodwin, M. H. (1987). Concurrent operations on talk: notes on the interactive organization of assessments. IPRA Papers Pragmatics 1, 1-52. doi: 10.1075/iprapip.1.1.01goo

Goodwin, C., and Goodwin, M. H. (1992). "Assessments and the construction of context," in Rethinking Context: Language as an Interactive Phenomenon, eds A. Duranti and C. Goodwin (Cambridge: Cambridge University Press), 147-190. 
Goodwin, C., and Goodwin, M. H. (2004). "Participation," in A Companion to Linguistic Anthropology, ed A. Duranti (Oxford: Blackwell), 222-244.

Hall, J. A., Roter, D., and Katz, N. R. (1987). Task versus socioemotional behaviours in physicians. Med. Care 25, 399-412. doi: 10.1097/00005650-198705000-00004

Han, P. K., Klein, W. M., and Arora, N. K. (2011). Varieties of uncertainty in health care: a conceptual taxonomy. Med. Decision Making 31, 828-838. doi: $10.1177 / 0272989$ X10393976

Heath, C. (1986). Body Movement and Speech in Medical Interaction. Cambridge: Cambridge University Press.

Heritage, J. (1984a). Garfinkel and Ethnomethodology. Cambridge: Polity Press.

Heritage, J. (1984b). "A change-of-state token and aspects of its sequential placement," in Structures of Social Action, eds J. Maxwell Atkinson and J. Heritage (Cambridge: Cambridge University Press), 299-345.

Heritage, J., and Maynard, D. (2006). Communication in Medical Care: Interaction Between Primary are Physicians and Patients. Cambridge: Cambridge University Press.

Holmes, J. (2000). "Doing collegiality and keeping control at work: small talk in government departments," in Small Talk, ed J. Coupland (Harlow: Pearson Education Limited), 32-61.

Hubbard, J., Illingworth, N., Rowa-Dewar, N., Forbat, L., and Kearney, N. (2010). Treatment decision-making in cancer care: the role of the carer. J. Clin. Nurs. 19, 2023-2031. doi: 10.1111/j.1365-2702.2009.0 3062.x

Ishikawa, K., Roter, D. L., Yamazaki, Y., and Takayama, T. (2005). Physicianelderly patient- of patient companions and their effect on decisionmaking activity in geriatric primary care visits. Soc. Sci. Med. 60, 1583-1591.

Jansen, J., van Weert, J., Wijngaards-deMeij, L., van Dulmen, S., Heeren, T. J., and Bensing, J. M. (2010). The role of companions in aiding older cancer patients to recall medical information. Psycho Oncol. 19, 170-179. doi: 10.1002/ pon. 1537

Jefferson, G. (1984). "On the organization of laughter in talk about troubles," in Structures of Social Action: Studies in Conversation Analysis, ed J. M. Atkinson and J. C. Heritage (Cambridge: Cambridge University Press), 346-369.

Jefferson, G. (2004). "Glossary of transcript symbols with an introduction," in Conversation Analysis: Studies From the First Generation, ed G. H. Lerner (Amsterdam: John Benjamins), 13-31.

Jefferson, G., Sacks, H., and Schegloff, E. A. (1987). "Notes on laughter in pursuit of intimacy," in Talk and Social Organization, eds G. Button and J. R. E. Lee (Clevedon: Multilingual Matters), 152-205.

Koenig, C. J. (2011). Patient resistance as agency in treatment decisions. Soc. Sci. Med. 72, 1105-1114. doi: 10.1016/j.socscimed.2011. 02.010

Laidsaar-Powell, R. C., Butow, P., Bu, S., Dear, R., Fisher, A., Coll, J., et al. (2016). Exploring the communication of oncologists, patients and family members in cancer consultations: development and application of a coding system capturing family-relevant behaviours (KINcode). Psychooncology 25, 787-794. doi: $10.1002 /$ pon. 4003

Laidsaar-Powell, R. C., Butow, P. N., Bu, S., Charles, C., Gafni, A., Lam, W. W. T., et al. (2013). Physician-patient-companion communication and decisionmaking: a systematic review of triadic medical consultations. Patient Educ. Counsel. 91, 3-13. doi: 10.1016/j.pec.2012.11.007

Lamore, K., Montalescot, L., and Untas, A. (2017). Treatment decisionmaking in chronic diseases: what are the family members roles, needs and attitudes? A systematic review. Patient Educ. Counsel. 100, 2172-2181. doi: 10.1016/j.pec.2017.08.003

Laryionava, K., Pfeil, T. A., Dietrich, M., Reiter-Theil, S., Hiddemann, W., and Winkler, E. C. (2018). The second patient? Family members of cancer patients and their role in end-of-life decision making. BMC Palliative Care 17:29. doi: $10.1186 / \mathrm{s} 12904-018-0288-2$

Laver, J. (1975). "Communicative functions of phatic communion," in Organization of Behavior in Face-to-Face Interaction, eds A. Kendon, R. M. Harris, and M. R. Key (The Hague: Mouton), 215-238.

Legare, F., Stacey, D., Briere, N., Robitaille, H., Lord, M. C., Desroches, S., et al. (2014). An interprofessional approach to shared decision making: an exploratory case study with family caregivers of one IP home care team. $B M C$ Geriatrics 14, 1-13. doi: 10.1186/1471-2318-14-83

Maynard, D., and Hudak, P. (2008). Small talk, high stakes: interactional disattentiveness in the context of prosocial doctor-patient interaction. Lang. Soc. 37, 661-688. doi: 10.1017/S0047404508080986

Mazer, B. L., Cameron, R. A., DeLuca, J. M., Mohile, S. G., and Epstein, R. M. (2014). "Speaking-for" and "speaking-as": pseudo-surrogacy in physicianpatient-companion medical encounters about advanced cancer. Patient Educ. Counsel. 96, 36-42. doi: 10.1016/j.pec.2014.05.001

Mitchell, J., Hawkins, J., Williams, E-D., Eggly, S., and Albrecht, T. (2019). Decoding the role of companions in supporting the health communication of older african-american men with cancer. J. Patient Exp. 7:237437351984409. doi: $10.1177 / 2374373519844098$

Mondada, L. (2006). Participants' online analysis and multimodal practices: projecting the end of the turn and the closing of the sequence. Discourse Stud. 8, 117-129. doi: $10.1177 / 1461445606059561$

Mondada, L. (2007). Multimodal resources for turn-taking. Discourse Stud. 9, 194-225. doi: 10.1177/1461445607075346

Mondada, L. (2018). Multiple temporalities of language and body in interaction: challenges for transcribing multimodality. Res. Lang. Soc. Interaction 51, 85-106. doi: 10.1080/08351813.2018.1413878

Mulders, M., Vingerhoets, A., and Breed, W. (2008). The impact of cancer and chemotherapy: perceptual similarities and differences between cancer patients, nurses and physicians. European J. Oncol. Nurs. 12, 97-102. doi: $10.1016 /$ j.ejon.2007.10.002

Nail, L. M. (2001). I'm coping as fast as I can: psychosocial adjustment to cancer and cancer treatment. Oncol. Nurs. Forum 28, 967-970.

Ochs, E., and Schieffelin, B. (1989). Language has a heart. Text 9, 7-25. doi: 10.1515/text.1.1989.9.1.7

Pauletto, F., and Fatigante, M., (2015) 'Dai', 'da' na mano!' Tra il dire e il chiedere: l'uso del segnale discorsivo 'dai' in conversazioni in famiglia. Riv. Ital. Psicoling. Appl. XV, 89-94. doi: 10.1400/232660

Pino, M., Doehring, A., and Parry, R. (2020). Practitioners' dilemmas and strategies in decision-making conversations where patients and companions take divergent positions on a healthcare measure: an observational study using conversation analysis. Health Commun. 22, 1-12. doi: $10.1080 / 10410236.2020 .1813952$

Pino, M., Fatigante, M., Zucchermaglio, C., and Alby, F. (2021). Two sources of miscommunication in oncology consultations: an observational study using conversation analysis. Appl. Ling. (in press).

Pino, M., and Parry, R. (2019). How and when do patients request life-expectancy estimates? Evidence from hospice medical consultations and insights for practice. Patient Educ. Counsel. 102, 223-237. doi: 10.1016/j.pec.2018.03.026

Robinson, J., and Stivers, T. (2001). Achieving activity transitions in primary-care encounters: from history taking to physical examination. Hum. Commun. Res. 27, 253-298. doi: 10.1111/j.1468-2958.2001.tb00782.x

Robinson, J. D. (2003). An interactional structure of medical activities during acute visits and its implications for patients' participation. Health Commun. 15, 27-59. doi: 10.1207/S15327027HC1501_2

Ruusuvuori, J. (2013). "Emotion, affect and conversation," in The Handbook of Conversation Analysis, eds J. Sidnell and T. Stivers (West Sussex: Blackwell Publishing Ltd), 331-349.

Ruusuvuori, J., and Peräkylä, A. (2009). Facial and verbal expressions in assessing stories and topics. Res. Lang. Soc. Interaction 42, 377-394. doi: 10.1080/08351810903296499

Sacks, H., Schegloff, E. A., and Jefferson, G. A. (1974). A simplest systematics for the organization of turn-taking in conversation. Language 50, 696-735. doi: 10.1353/lan.1974.0010

Schegloff, E. (1968). Sequencing in conversational openings. Am. Anthropol. 70, 1075-1095. Available online at: http://www.jstor.org/stable/669510

Schegloff, E., Jefferson, G., and Sacks, H. (1977). The preference for selfcorrection in the organization of repair in conversation. Language 53, 361-382. doi: $10.2307 / 413107$

Schegloff, E. A. (1980). Preliminaries to preliminaries: "Can I ask you a question?". Sociol. Inquiry 50, 104-152. doi: 10.1111/j.1475-682X.1980.tb00018.x

Schegloff, E. A. (1995). "Parties and joint talk: two ways in which numbers are significant for talk-in-interaction," in Situated Order Studies in the Social 
Organization of Talk and Embodied Activities, eds P. ten Have and G. Psathas (Washington, DC: University Press of America), 31-42.

Schegloff, E. A. (1998). Body torque. Social Res. 65, 536-596.

Schegloff, E. A. (2000). Overlapping talk and the organization of turn-taking for conversation. Lang. Soc. 29, 1-63. doi: 10.1017/S0047404500001019

Schegloff, E. A. (2007). Sequence Organization in Interaction: A Primer in Conversation Analysis I. Cambridge: Cambridge University Press.

Shepherd, H., Tattersall, M., and Butow, P. (2007). The context influences doctors' support of shared decision-making in cancer care. Br. J. Cancer 97, 6-13. doi: $10.1038 /$ sj.bjc.6603841

Singer S. (2018) Psychosocial Impact of Cancer. Recent Results Cancer Res. 210:111. doi: 10.1007/978-3-319-64310-6_1

Sterponi, L., Zucchermaglio, C., Alby, F., and Fatigante, M. (2017). Endangered literacies? Affordances of paper- based literacy in medical practice and its persistence in the transition to digital technology. Written Commun. 1-28. doi: $10.1177 / 0741088317723304$

Stivers, T. (2008). Stance, alignment and affiliation during story telling: nodding as a token of preliminary affiliation. Res. Lang. Soc. Interaction 41, 31-57. doi: 10.1080/08351810701691123

Stivers, T., and Rossano, F. (2010). Mobilizing response. Res. Lang. Soc. Interaction 43, 3-31. doi: 10.1080/08351810903471258

Street, R. L., and Gordon, H. S. (2008). Companion participation in cancer consultations. Psychooncology 17, 244-251. doi: 10.1002/pon.1225

Tate, A., and Rimel, B. (2020). The duality of option-listing in cancer care. Patient Educ. Counsel. 103, 71-76. doi: 10.1016/j.pec.2019.07.025
Wolff, J. L., Guan, Y., Boyd, C. M., Vick, J., Amjad, H., Roth, D. L., et al. (2017). Examining the context and helpfulness of family companion contributions to older adults' primary care visits. Patient Educ. Counsel. 100, 487-494. doi: $10.1016 /$ j.pec.2016.10.022

Wolff, J. L., and Roter, D. L. (2008). Hidden in plain sight: medical visit companions as a resource for vulnerable older adults. Arch. Internal Med. 168, 1409-1415. doi: 10.1001/archinte.168.13.1409

Wolff, J. L., and Roter, D. L. (2011). Family presence in routine medical visits: a meta-analytical review. Soc. Sci. Med. 72, 823-831. doi: 10.1016/j.socscimed.2011.01.015

Zucchermaglio, C., Alby, F., and Fatigante, M. (2016). What counts as illness? Anamnesis as a collaborative activity. Test. Psychometr. Methodol. Appl. Psychol. 23, 471-487. doi: 10.4473/TPM23.4.4

Conflict of Interest: The authors declare that the research was conducted in the absence of any commercial or financial relationships that could be construed as a potential conflict of interest.

Copyright (C) 2021 Fatigante, Zucchermaglio and Alby. This is an open-access article distributed under the terms of the Creative Commons Attribution License (CC BY). The use, distribution or reproduction in other forums is permitted, provided the original author(s) and the copyright owner(s) are credited and that the original publication in this journal is cited, in accordance with accepted academic practice. No use, distribution or reproduction is permitted which does not comply with these terms. 


\section{APPENDIX A}

\section{Transcription Conventions (Jefferson et al.,} 1987; Mondada, 2018)

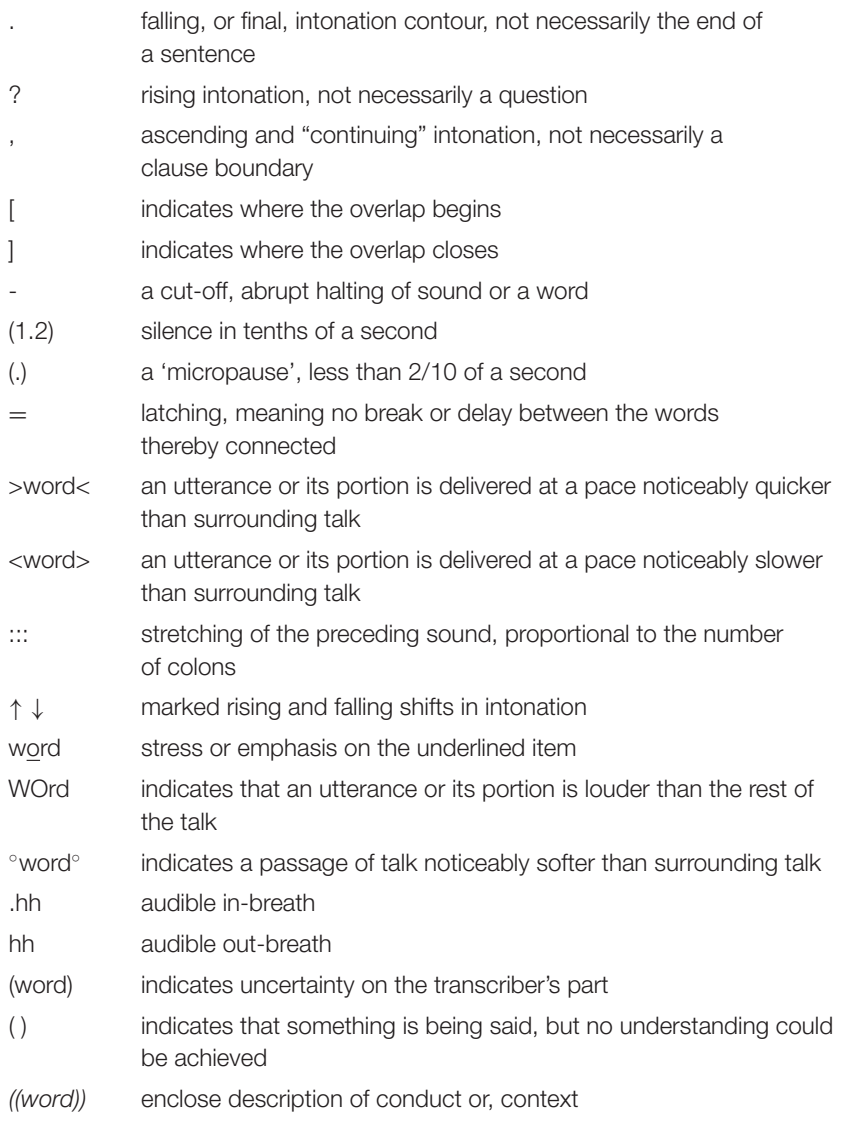

\section{Notation for Visible Actions}

* $\quad$ points where the doctor's visible behavior (e.g., a nod) starts or ends

$\S \quad$ points where the patient's visible behavior (e.g., a nod) starts or ends

$\% \quad$ points where the companion's visible behavior (e.g., a nod) starts

or ends

$\rightarrow \quad$ the action described continues across subsequent lines 
OPEN ACCESS

Edited by:

Antonio Bova,

Catholic University of the Sacred

Heart, Italy

Reviewed by:

Dimitris Serafis,

University of Malta, Malta

Antonia Larrain

Alberto Hurtado University, Chile

*Correspondence:

María Pilar Jiménez-Aleixandre

marilarj.aleixandre@usc.gal

${ }^{\dagger}$ These authors have contributed equally to this work and share first authorship

Specialty section

This article was submitted to

Language Sciences,

a section of the journal

Frontiers in Psychology

Received: 31 January 2021

Accepted: 10 May 2021

Published: 08 June 2021

Citation:

Jiménez-Aleixandre MP and Brocos $P$ (2021) Emotional Tension as a Frame

for Argumentation and Decision-Making: Vegetarian vs.

Omnivorous Diets.

Front. Psychol. 12:662141 doi: 10.3389/fpsyg.2021.662141

\section{Emotional Tension as a Frame for Argumentation and Decision-Making: Vegetarian vs. Omnivorous Diets}

\author{
María Pilar Jiménez-Aleixandre ${ }^{\star \dagger}$ and Pablo Brocos ${ }^{\dagger}$ \\ Departamento de Didácticas Aplicadas, Universidade de Santiago de Compostela, Santiago de Compostela, Spain
}

Argumentative discourse has a complexity that is not entirely captured by purely structural analyses. In arguments about socio-scientific issues (SSI), a range of dimensions, besides scientific knowledge, including values, ethical concerns, cultural habits, or emotions, are mobilized. The relationship between argumentation and emotions is now drawing attention of researchers. Our focus is on the dynamic interactions among emotions and scientific evidence. We draw from Plantin, who proposed that emotions are mobilized as argumentative resources alongside knowledge. The goal of our study is to examine in which ways emotional tension frames the construction of arguments about vegetarian vs. omnivorous diets (ODs) with a group of four preservice teachers. The results suggest that the interactions between the group emotional tension and the evaluation of evidence drive a change toward a decision that would be emotionally acceptable for all participants. Participants attended to the epistemic dimension, weighing evidence, and values about the choices, but the emotional framing took priority. We suggest that the analysis of this emotive framing may be a fruitful approach for sophisticated studies of argumentation beyond structural issues.

Keywords: argumentation, emotions, discourse, decision-making, vegetarianism, sustainable diet

\section{INTRODUCTION: ARGUMENTATION AND EMOTIONS ABOUT DIETS}

The analysis of the structure of arguments-in other words, the number, quality, and relationships between components such as claims, data, justifications, or rebuttals-has yielded relevant insights about how knowledge is justified or, more generally, evaluated (e.g., Berland and McNeill, 2010; Osborne et al., 2016; Bravo-Torija and Jiménez-Aleixandre, 2018). Argumentative discourse has, however, a complexity that is not entirely captured by purely structural analyses. In particular, in arguments about socio-scientific issues (SSI), a range of dimensions, besides scientific knowledge, including values, ethical concerns, cultural habits, or emotions, are mobilized. The relationship between argumentation and emotions, understudied for years, is recently drawing attention of researchers (e.g., Micheli, 2010; Baker et al., 2013a; Pollaroli et al., 2019). Previous work on argumentation about SSI addressed this relationship in terms of distinctions among types of arguments, for instance, Zeidler and Sadler's (2008) description of three argumentative patterns: rational, intuitive, and emotive. This study is framed in a different perspective, for we are interested in the dynamic interactions among emotions, evaluation of scientific evidence, and other dimensions such as cultural identities or ethical concerns, what for Hufnagel $(2015,2019,2021)$ 
constitutes emotional sense-making. Plantin (2011) took a novel approach to the relationships between argumentation and emotions, conceiving emotions as argumentative resources that are mobilized alongside knowledge. We draw from Plantin's (2019) work about variation of interactional tension as a defining feature of emotions in discourse, combining it with Andriessen et al.'s (2011) approach to socio-cognitive tension in argumentative interactions. The balance between engagement in argumentation and sustaining favorable socio-emotional processes has been explored by Isohätälä et al. (2018). By socio-emotional processes, they mean the social and emotional dimensions of collaborative learning: social "in the sense that they are dynamically created within the interpersonal setting through the social interactions that the learners engage in" (p. 2); and emotional in the sense that the learners' perceptions of the social context are related to their emotions. Isohätälä et al. (2018) claim that notions as socio-emotional processes or relational space are attempts to capture the efforts of participants to sustain cohesive social interactions.

Building on these approaches to the role of emotions, our study seeks to add knowledge to argumentation studies by examining how preservice teachers build emotional tension while engaged in argumentation in the context of decisionmaking about the dilemma of omnivorous vs. vegetarian diets (VDs). This SSI context was chosen by considering the growing concerns on how to feed global population (Food and Agriculture Organization of the United Nations (FAO), 2009), the longterm effect of diets on health (International Agency for Research on Cancer, 2015), and the environmental impact of human nutrition. Although for decades the environmental focus has been on energy sources and uses, a growing area of research assesses the impact of diets on sustainability (Stehfest et al., 2009; Thompson et al., 2013; Tilman and Clark, 2014; Hyland et al., 2017) and on climate change. The Intergovernmental Panel on Climate Change (IPCC, 2019) has issued a special report, including a chapter on food security, which attributes about $21-37 \%$ of total greenhouse gas emissions to the food system. In order to reduce this impact, the report suggests that healthy and sustainable diets should be high in vegetables and low in animal products, such as meat, while acknowledging that dietary changes are guided by social, cultural, environmental, and traditional factors; and hence recognizing the influence of affective dimensions related to culture and traditions. It should be noted that environmental disruption and intensive livestock production are also identified as part of the causes involved in the cross-species transmission of the coronavirus in the coronavirus disease of 2019 (COVID-19) pandemic (Cui et al., 2019; IPBES, 2020); as Zheng-Li Shi, a leading expert on coronavirus diseases, claimed on her WeChat: "The 2019 novel coronavirus is a punishment by nature to humans' unsanitary life styles."

Against this backdrop, the goal of our study is to examine in which ways emotional tension frames the construction of arguments about vegetarian vs. omnivorous diets (ODs) using preservice teachers. The research question is:

In which way does emotional tension act as a process of framing in an argumentative debate?
First, we discuss recent approaches to the role of emotions in argumentation; second, we present the data sources and methods; and third, we discuss the findings and their potential research and educational implications.

\section{RATIONALE: EMOTIONS AND ARGUMENTATION}

Argumentation and decision-making about SSI deal with complex issues, demanding the consideration of different dimensions and drawing from multidisciplinary perspectives. In many cases, these arguments address open-ended dilemmas as they do not have a "best" solution that can meet all requirements from different dimensions. As Morin et al. (2014) pointed out, these open-ended issues bring out the complexities and uncertainties embedded in ill-structured problems, reflecting social representations and value systems. In arguments about SSI, a range of dimensions, besides scientific knowledge, such as values, ethical concerns, cultural habits, or emotions, are mobilized. Thus, SSI contexts can be suitable for the study of emotions as argumentative resources (Polo, 2014). We draw from the communication studies that define the framing as a rhetorical process through which communicators (consciously or unconsciously) construct a point of view to promote a particular interpretation of facts (Kuypers, 2009). Our goal is to explore how emotional tension may act as a process of framing in an argumentative debate. Under these premises, first, we discuss research about argumentation and emotions, and second, the relevance of discursive contexts in argumentation.

\section{Argumentation and Emotional Tension}

Aristotle, in his Rhetoric, underlined that emotions are among the three means of persuasion (ethos, pathos, and logos), and nowadays it is generally acknowledged that argumentation involves both justification and persuasion (Jiménez-Aleixandre and Erduran, 2008). However, the relationship between argumentation and emotions has been, for years, an understudied issue. Emotions were absent in the seminal work of Toulmin (1958), one of the foundations for the study of argumentation in science education. This gap may be related to a bias in learning research toward the cognitive at the expenses of the affective (Baker et al., 2013b). Thus, Baker et al. (2013a) pose questions such as how do interpersonal relations, the circulation of emotions, and collaborative learning interrelate. In his pioneer work about emotions, Plantin (2011) questioned the antagonism between reason and emotion, with origins in the stoic philosophers, pointing out that they are inseparable. It should be noted that in the 17th century Baruch Spinoza (1677/2012) challenged the Cartesian mindbody dualism, suggesting a relationship between emotions and rational decisions, a challenge that caused his exclusion from the Jewish community. The relevance of Spinoza's ideas is being reappraised in the field of psychology of emotions (Brown and Stenner, 2001).

An increasing interest in the role of the emotions on the argumentative discourse has arisen, particularly in the French 
linguistic tradition, drawing from the work of Grize (1996) and his notion of schematization, a discursive representation of discourse objects. Within this tradition, there has been a distinction between emotional, the authentic psychological emotions the participants may feel, and emotive, what is discursively expressed. However, there is no evidence that expressed and felt emotions are actually different (Polo et al., 2017; Herman and Serafis, 2019), so we will use them interchangeably. According to Voloshinov (1986), the relationship between discourse and emotions is dialectical, and utterances imply both an evaluative stance toward interlocutors and an emotional positioning. By discourse, drawing from a sociolinguistic perspective, we mean not merely language in use, but language situated in ongoing sociocultural practices with histories, discourses, intertextual references, and social relationships of members of the relevant social group (Kelly, 2021).

It should be noted that the focus of our study is neither on determining participants' felt emotions, nor on the validity of their arguments, but rather on understanding how they mobilize the emotions as argumentative resources for argumentative purposes within the discourse, as proposed by Plantin (2011), and how, in doing so, they sustain socio-emotional interactions (Isohätälä et al., 2018). This entails methodological challenges as emotions can be present in multiple forms in the discourse, being frequently implicit (Herman and Serafis, 2019). Plantin (2011) suggested a need for moving beyond the lexical analysis in order to reach the implicit emotional component of the discourse through the inferences built on cultural stereotypes. We draw from his work about tension in argumentation (Plantin, 2019) to analyze the role of emotions in participants' argumentative exchanges in terms of what we call emotional tension. He characterized tension, from the point of view of argumentation, as "an operator showing that the speaker is highly involved in her speech and wants to share her commitments, that is, wants to persuade her audience" (Plantin, 2019, p. 348). Furthermore, he pointed out that "Tension variation is the defining feature of emotions in general-in discourse as in interactions" (ibidem, p. 362; Plantin's italics). Therefore, we suggest that emotional tension is an adequate term for the analysis of these interactions, as it was in agreement with Plantin (personal communication). The analytical framework and the coding categories derived from it are discussed in the "Methods" section.

Framing their work in collaborative learning, Baker et al. (2013a) addressed the interrelationships among emotions, interpersonal relations, and learning in their edited volume, with one section devoted to argumentation and emotion. Within it, Muller Mirza (2013) focused on the ways interlocutors in argumentative discussions may interpret or frame disagreements and conflicts, in other words on the conflictual dimension of argumentation. She examined how these conflicts may foster or hinder epistemic argumentation, suggesting elements for the design of a "thinking space," which creates opportunities for the integration of identity and affective processes in argumentative practices. The relationships between epistemic, what participants are saying, and interpersonal, how they say it, dimensions of argumentation are the focus of Asterhan's (2013) chapter, also paying particular attention to different attitudes toward conflict resolution, which result in two types of argumentative discourse, consensus seeking and adversarial. Asterhan (2013, p. 255) pointed out how affective concerns "may divert students' attention away from the epistemic dimension of the conflict (a conflict between ideas) and heavily focus on the interpersonal dimension of the conflict (a conflict between persons)." This may result in argumentative discourses either void of the critical dimension, because the participants seek a quick consensus, or void of collaborative knowledge construction (adversarial). In the first case, disagreement with peers may be perceived as hampering positive relationships and acceptation. She suggested the interest of engaging students in the discussions that are both critical and co-constructive, otherwise they may experience difficulties in combining these dimensions. This balance, or lack thereof, is relevant for our study. Argumentative discourse is a sociocultural practice; Brown and Stenner (2001) discuss the significance of Spinoza's ideas for the studies about the social construction of emotions; for instance, the tension between "what might be characterized as 'materiality' and 'the work of thought"' (p. 98), where materiality corresponds to bodies and thought to minds. Personal emotional processes are also in play when engaging in argumentation; for instance, Richter and Maier (2017) examine how readers' prior beliefs may lead to a biased processing of conflicting information. The meaning of "beliefs" in the context of the task is discussed below.

The place of identity and emotions in argumentative learning has been addressed by Schwarz and Goldberg (2013). In line with Plantin's (2011) approach, they considered identity and emotions as resources in historical reasoning. Identity conflicts in historical argumentation of Spanish (Galician) high school students have been examined by López-Facal et al. (2015), showing how national identification influenced their arguments, which were different when the discourse object was Ireland from when it was the Basque Country, a historical national within Spain that was perceived as affecting their own national identity. In our study, participants' Galician identity, mostly implicit, is appealed to in their arguments as discussed in the findings. Although science issues have been considered "cold" and unaffected by emotions, science education research has shown the influence of motivational and affective factors (Darner, 2019). Darner, focusing on science denial, called for a recognition of science topics as emotionally laden, which is the case of dietary choices. In science education, Avraamidou (2020) discussed the process of forming a science identity, with an emphasis on recognition and emotions, and she suggested that emotions can offer a valuable lens for studying inequalities. In Hufnagel (2021), identity is a reference point for emotions. In her program of research about emotional sense-making in the context of climate change, emotions are conceived as evaluative mechanisms that indicate personal relevance and deep relationship to ideas or objects (Hufnagel, 2015). Her model for the analysis of emotions considered them as social and situated, pointing out that "emotions are not internal entities but a relationship to a specific event, experience, idea, and so forth" (Hufnagel, 2019, p. 156). For our study, the identity that matters is being Galician, on two dimensions: first, the evaluation of the impact of the adoption 
of a VD in Galician economy, where breeding is very important; second, on the consideration of eating meat as part of the cultural identity of participants.

Being relevant to our work, Isohätälä et al.'s (2018) study discussed about how student teachers, in the context of a teacher education course on environmental science, struck a balance between engaging in argumentation and sustaining socio-emotional processes favorable to it. They found that the groups sustained favorable socio-emotional processes, but mostly failed to engage in argumentation. The participants generally refrained from critical discussions, accepting each other's claims or conceding to divergent claims without argumentation; the challenging nature of argumentation may cause participants to attend to socio-emotional processes at the expense of cognitive ones. This is a question pointed out by Asterhan (2013) as discussed above. The authors suggested the need for more studies from authentic "messy" learning contexts.

As a summary, there is a growing interest in the intertwining of emotional and cognitive processes in argumentation, a body of research to which this study seeks to contribute. Our focus is on how emotional tension frames argumentative processes as it is one of the ways of exploring the role of emotions in argumentation.

\section{Discursive Context of Decision-Making}

Jiménez-Aleixandre and Brocos (2018) suggested that argumentative operations and products are likely to differ depending on discursive contexts specific to pedagogical discursive practices, such as constructing and evaluating causal explanations, or making decisions, which is the one addressed in this study. This is an analytical frame conceived for research purposes, as in actual classroom settings these contexts may overlap. While in the construction and evaluation of causal explanations and models the aim is to choose the model that is best supported by evidence, decision-making is characterized by the use of evidence in order to make a decision or to choose a course of action. There is a set of common operations, for instance, using appropriate criteria for identifying and evaluating genuine evidence; identifying which evidence is valid and relevant for the issue at stake; considering multiple claims, theories, or options; or engaging with each other's ideas, supporting or challenging them. However, because cognitive and emotional processes are situated, other operations are particularly relevant in a given context. For instance, generating rebuttals is of relevance to eristic settings when two contrasted arguments, sometimes about emotionally charged issues, are opposed. However, in cooperative work in small groups, participants need to be able to conceptualize arguments different from their own, and a mark of quality may be the co-construction of arguments among several participants (Jiménez-Aleixandre et al., 2000). A distinction between contexts may be that, in decision-making, the discursive path can proceed from evidence to claims, as, for instance, in the study of Bravo-Torija and Jiménez-Aleixandre (2018); while in the evaluation of causal explanations the discussion proceeds, in many cases, from the alternative claims to the evidence supporting them (JiménezAleixandre et al., 2000). In their work about shifts in epistemic status in argumentation, Jiménez-Aleixandre and Brocos (2018) suggested that in the context of developing explanations and models, the focus is rather on the individual learner whereas in decision-making the focus is on the participants in a social interaction. Furthermore, while plausibility is a relevant feature in evaluating explanations and models, in decision-making the focus is on acceptability "which indicates not only the degree of feasibility of the options considered, in light of the available evidence and previous ideas, but also their accordance with personal and social values" (Jiménez-Aleixandre and Brocos, 2018, p. 174-175). Thus, for instance, participants may believe or not that carrying out a specific option-as, in this study, the VD-it is individually or socially possible; and for doing so they have to take into account if the option is consistent or not consistent with other conceptions and values individually or socially accepted, such as those attached to different cultures and traditions in a given context. These beliefs in the plausibility of a given option may influence how they evaluate information (Richter and Maier, 2017), while their Galician identity is a reference point for emotions (Hufnagel, 2021), and may increase the emotional tension in this decision-making context.

\section{DATA SOURCES AND METHODS}

\section{Methodological Approach, Participants, and Learning Context}

This study adopts a qualitative method approach, seeking to analyze educational case studies through expressions and actions in their local contexts (Denzin and Lincoln, 2013). Qualitative approaches are appropriate to study processes and evaluation practices (Creswell, 2013). It makes part of a wider study with 85 preservice primary teachers (PST). Studies on the challenges experienced by teachers for supporting their students' engagement in argumentation and SSI are limited, in comparison with the studies on students (Evagorou and Puig, 2017). In this paper, we analyze the arguments of a small group of four PST in which all participants agreed to be recorded. They were enrolled in a science education course taught by the first author, engaging in tasks about the evidence evaluation, criteria for strong arguments, and balanced diets; they sought the information about dimensions (environmental, ethical, nutritional, economic, or cultural) of diets, shared through a wiki, and constructed arguments in small groups, formed by them, about sustainable and healthy diets. The design of the teaching sequence is discussed in detail by Brocos and JiménezAleixandre (2020). Ours is a bilingual context, where both coofficial languages, Galician and Spanish, are used interchangeably and fully understood by all actors. The texts and debates have been translated into English by the authors.

Participants are identified with pseudonyms, beginning with the letter of their small group. In group $B$, there were three males and a female (Bea); their ages in years being 21 (Borja), 24 (Bea), 38 (Breixo), and 43 (Blas). While the ages of the two younger ones were in correspondence to the mean age of the whole class, the other two were significantly older. 
TABLE 1 | Coding categories: variations in emotional tension (drawing from Andriessen et al., 2011; Plantin, 2011, 2019).

\begin{tabular}{|c|c|c|c|}
\hline Tension & Categories & Characterization & Examples \\
\hline \multirow[t]{5}{*}{$\begin{array}{l}\text { High emotional } \\
\text { tension }\end{array}$} & Life-death & $\begin{array}{l}\text { References to life are framed as positive, } \\
\text { references to death as negative. }\end{array}$ & $\begin{array}{l}292 \text { Blas: you can't die yourself to save the } \\
\text { animals, mate. }\end{array}$ \\
\hline & Interjections/exclamations & $\begin{array}{l}\text { Exclamatory statements, cursing, rising } \\
\text { intonation. }\end{array}$ & 74 Blas: Damnit! \\
\hline & Interruptions & Participants interrupting each other. & $\begin{array}{l}225 \text { Breixo [interrupting] But, I mean, } \\
\text { implicitly... }\end{array}$ \\
\hline & Radicalization & $\begin{array}{l}\text { All-or-nothing arguments rejecting } \\
\text { compromises, excluding counter-discourse. }\end{array}$ & $\begin{array}{l}124 \text { Blas: For instance, veganism seems } \\
\text { aberrant to me. }\end{array}$ \\
\hline & Rhetorical questions & $\begin{array}{l}\text { Questions that both challenge and give no } \\
\text { voice to the opponent; pretend to express a } \\
\text { shared knowledge. }\end{array}$ & $\begin{array}{l}344 \text { Blas: it has always been more ethical to } \\
\text { hunt a rabbit in the wild than keeping } 20,000 \\
\text { rabbits there locked up, all dejected, isn't it? }\end{array}$ \\
\hline \multirow[t]{5}{*}{$\begin{array}{l}\text { Medium emotional } \\
\text { tension }\end{array}$} & Distance/closeness & $\begin{array}{l}\text { Place distance/closeness, and people } \\
\text { distance/closeness (identity). }\end{array}$ & $\begin{array}{l}35 \text { Bea: if [vegetarian diet] is for many people, } \\
\text { then... Galician economy would be damaged }\end{array}$ \\
\hline & Taking stance & Expression of a position, claim. & $\begin{array}{l}46 \text { Blas: It is a diet completely in favor of meat } \\
\text { consumption }[\ldots]\end{array}$ \\
\hline & Counterclaim & Opposition to a claim. & $\begin{array}{l}116 \text { Breixo: No, lactovegetarian you don't eat } \\
\text { meat. }\end{array}$ \\
\hline & Requesting clarification & Asking about meaning/about evidence. & $\begin{array}{l}156 \text { Breixo: Omnivorous diet. Arguments for it? } \\
\text { Which are your data? }\end{array}$ \\
\hline & Impact/Consequences & $\begin{array}{l}\text { Desirability or not of the consequences (real or } \\
\text { expected) of the situation. }\end{array}$ & $\begin{array}{l}26 \text { Bea: But if everybody chooses the } \\
\text { vegetarian option, it would affect Galicia } \\
\text { negatively. }\end{array}$ \\
\hline \multirow[t]{5}{*}{$\begin{array}{l}\text { Low emotional } \\
\text { tension/Negotiation }\end{array}$} & Seeking compromise, negotiating & $\begin{array}{l}\text { Looking for a position that would be } \\
\text { acceptable for both sides. }\end{array}$ & 132 Bea: You can also be partially vegetarian. \\
\hline & Summarizing & Recapitulating information or positions. & $\begin{array}{l}147 \text { Blas: environmental impact [of meat diet] } \\
{[\ldots] \text { more greenhouse gases }}\end{array}$ \\
\hline & Focusing, Building & $\begin{array}{l}\text { Directing debate to the task goals and } \\
\text { adequate procedures }\end{array}$ & $\begin{array}{l}275 \text { Breixo: Yes, I agree, but... we need to } \\
\text { support it in argument. }\end{array}$ \\
\hline & Clarifying & Explaining meanings or positions & 154 Blas: Fish... maybe. \\
\hline & $\begin{array}{l}\text { Acknowledging } \\
\text { contributions, confirming, agreeing }\end{array}$ & Recognizing or validating ideas and inputs, & $\begin{array}{l}218 \text { Breixo: Fine, [what Blas said] then I write... } \\
\text { meat industry would be reduced, and } \\
\text { compensated with increase in agro-industry. }\end{array}$ \\
\hline
\end{tabular}

Participants were asked to construct an argument about which diet they would consider to be better. The handout is reproduced in Annex 1 (see Supplementary Materials). In order to build that argument, they were directed to use a complex data set, consisting of their own selection of information, collected in a wiki, as well as five additional handouts elaborated by the researchers, one for each dimension (cultural, environmental, economic, ethical, and nutritional). These additional handouts were produced to ensure that for each dimension there is available information supporting different and even conflicting choices (Jiménez-Aleixandre et al., 2019).

\section{Data Collection and Rubric for Analysis}

Data collection, through immersion of the second author as a participant observer, included participants' written products (individual pretest, portfolios, group final essay), video recording, and semi-structured interviews with three of the four participants (Blas, 32:54; Bea, 40:54; and Breixo, 48:3) 1 year later, after they had read the full transcription of the debate. For the purpose of this paper, the corpus comprises the video recording of a 90-min session devoted to construct the argument, their final essay, and the interviews.
Discourse was analyzed by using constant comparative analysis (Glaser and Strauss, 1967). The unit of analysis is the turn of speech, defined as each intervention by the participants. Turns were grouped into episodes and defined as one or several turns of speech related to the same topic or action (Gee, 2014); in this group we grouped turns into 10 episodes. Rubrics and coding categories emerged from the interaction of the theoretical frame with data in successive iterations. Transcriptions of the oral debate and the written essay were analyzed by both authors, initial repertoires of categories drawing from the literature were elaborated, and tentative codes were independently assigned to each unit.

Emotional tension was examined, primarily by analyzing its variations, and secondarily by considering the use of themes carrying affective weight. In order to analyze the variations in emotional tension, we constructed a repertoire, synthesized in Table 1, distributing the coding categories in High $(\mathrm{H})$, Medium (M), and Low (L) emotional tension. The repertoire draws from:

- Plantin (2019) components of tension, such as radicalization of arguments $(\mathrm{H})$, interjections $(\mathrm{H})$, and rhetorical questions $(\mathrm{H})$. 
- Plantin (2011) categories for emotional positioning, which he considers an axis for the emotive construction of the discourse, representing the evaluation of a discourse object on a pleasantunpleasant continuum, such as life-death $(\mathrm{H})$ and impactconsequences $(\mathrm{M})$. Also, we include one category for emotional intensity - a second axis for the emotive constructiondistance $(\mathrm{M})$, on a continuum from close to far, which may refer to a place or to the personal distance of participants.

- Andriessen et al.'s (2011) criteria for socio-cognitive tension and relaxation, such as interrupting $(\mathrm{H})$, taking stance $(\mathrm{M})$, counterclaim $(\mathrm{M})$, requesting clarification $(\mathrm{M})$, compromise $(\mathrm{L})$, focusing, and building (L).

- Authors' categories, such as summarizing (L), clarifying (L), and confirming, acknowledging contributions from others (L).

It should be noted that the distribution of categories for emotional tension in three levels is a simplification; on one hand, some categories are better seen as a continuum between increasing and decreasing tension. On the other hand, the significance of certain utterances is dependent on the task and the specific moment-to-moment interactions. The purpose is to capture the overall tension of each episode. Plantin (2011) identified the cultural conventions that emotionally frame the discourse. For instance, we can argue that conceptualizing an event as being close in terms of place, like it is the case with Galicia in this study, leads to stronger emotional response. Similarly, presenting a situation as going against widely recognized social identities (people closeness) may lead to an unpleasant emotional atmosphere. The categories for variations in emotional tension and their characterization are summarized in Table 1, with instances from our data.

The emotive framing of the issue, characterized through these categories, configures a certain emotional atmosphere for the debate, from higher tension, more eristic, to lower tension, associated with negotiation.

The use of themes carrying affective weight was analyzed by drawing from Plantin (2011), Polo (2014), and from the following two categories developed by Hufnagel $(2015,2019)$ : aboutness, referring to the objects of emotion, and type of feeling. Kerbracht-Orecchioni (1980) discussed affective substantives and adjectives, which enunciate both features of the object as well as "une réaction emotionnelle du sujet parlant en face de cet object" (p. 84). Supplementary Table 1 summarizes the themes appealed to by each participant. The selected themes satisfied either of the following criteria: (a) three or more mentions; (b) strong intensity; or (c) pleasant-unpleasant positioning. On the intensity axis, the more frequent theme carrying emotive weight was "supplements," with 23 mentions; the theme "pills" was mentioned in five occasions, and it was coded separately because of its semantic association with medicine and illness. Second in frequency is a cluster of "meat reduction," with 11 mentions, opposed by the claim that VD implies no meat at all (7). Closeness of the participants evidenced, for instance, by the use of first and second person, and by direct references to Galicia, was also used.

On the emotive positioning axis, "ethical" has the highest frequency, 21 references, although, as discussed below in relation to episode four, in some cases it seems a ritual invocation rather than a deep reflection on the issue. Second and third in frequency are two clusters: the second, situated on the pleasant side, concerns "argument," "evidence," and "criteria," with 19 references, expressing alignment with the norms for good arguments; the third, around "death," "to kill," and "slaughterhouse," is located on the unpleasant side, with nine references.

\section{DATA ANALYSIS-FINDINGS: FLUCTUATIONS OF EMOTIONAL TENSION}

Analyzing the variations in emotional tension in the argumentative debate provided opportunities for a better understanding of the intertwining of cognitive and emotive processes. Before describing the findings, we summarize the relative participation of the four group members, which was uneven. There are 356 turns of speech in the session, 348 leaving out eight clarifications by the instructor and the researcher (first and second author). From these, Borja made only nine, while the other three had, respectively, 125 (Blas), 122 (Breixo), and 92 (Bea). Borja's utterances were barely substantive, asking questions about task procedures or expressing agreement; for this reason, the analysis emphasizes the conversation of the three members who contributed significantly.

\section{Fluctuation of Emotional Tension Across Episodes}

We begin with an overview of the emotional tension across episodes. Table 2 summarizes the distribution of the session in episodes, the content or topics of each episode, and the number of $\mathrm{H}, \mathrm{M}$, and $\mathrm{L}$ tension turns in each one. As the number of turns is uneven, we provide the percentage in order to give an idea of the emotional climate in the episodes. It may be observed that, for 6 out of the 10 episodes, more turns-from 74.3 to $41.2 \%$-are coded as L; in one episode, L and M are tied; in two episodessecond and ninth-M are more frequent; and, in the last episode, $\mathrm{H}$ dominates. In other words, tension tends to increase as the debate moves forward.

\section{Central and Supporting Arguments}

There is a central argument running across the 10 episodes, which opposes the two options or clusters of options, OD and omnivorous reducing meat (OR) vs. vegetarian (VD) and vegan (VG) diets. OD was proposed and justified by Blas and Bea, with support from Borja; and VD by Breixo, who appealed to evidence pointing to the benefits of VD for health, the environment, and animal well-being. He sometimes defended VG, rather for the sake of the argument, considering that it was not his personal option, as he made explicit in the interview. The OD proposal, from episodes one to three, evolved to OR when Blas (112) suggested it.

The other five arguments are considered as secondary, supporting the central one. From these, the more frequent, in six episodes, dealt with the social vs. personal character of the choice, justifying $\mathrm{OD}$ on the basis that $\mathrm{VD}$ would not be 
TABLE 2 | Level of tension in each episode.

\begin{tabular}{|c|c|c|c|c|c|}
\hline Episode (turns) & Content & Coded turns & Emotional tension & \# turns & $\%$ \\
\hline \multirow[t]{3}{*}{$1(1-48)$} & Agreeing on task goals & 43 & $\mathrm{H}$ & 0 & - \\
\hline & & & M & 14 & 32.68 \\
\hline & & & L & 29 & 67.4 \\
\hline \multirow[t]{2}{*}{$2(49-87)$} & Discussing nutrition data & 39 & $\mathrm{H}$ & 5 & 12.8 \\
\hline & & & M & 21 & 53.8 \\
\hline \multirow{2}{*}{3 (88-105) } & & & M & 5 & 29.4 \\
\hline & & & L & 7 & 41.2 \\
\hline \multirow[t]{3}{*}{$4(106-146)$} & Proposing intermediate options: OR & 39 & $\mathrm{H}$ & 8 & 20.5 \\
\hline & & & M & 13 & 33.3 \\
\hline & & & L & 18 & 46.2 \\
\hline \multirow[t]{3}{*}{$6(180-220)$} & Weighing economic impact of VD for Galicia & 39 & $\mathrm{H}$ & 1 & 2.6 \\
\hline & & & M & 9 & 23 \\
\hline & & & L & 29 & 74.3 \\
\hline \multirow[t]{3}{*}{$7(221-247)$} & Negotiating OR option & 25 & $\mathrm{H}$ & 7 & 28 \\
\hline & & & M & 9 & 36 \\
\hline & & & L & 9 & 36 \\
\hline \multirow[t]{3}{*}{$8(248-280)$} & Discussing ethical dimension & 23 & $\mathrm{H}$ & 5 & 21.7 \\
\hline & & & M & 7 & 30.5 \\
\hline & & & L & 11 & 47.8 \\
\hline \multirow[t]{2}{*}{$9(281-337)$} & Disagreement OR vs. VD & 49 & $\mathrm{H}$ & 14 & 28.6 \\
\hline & & & M & 19 & 38.8 \\
\hline
\end{tabular}

OD, Omnivorous Diet; OR, Omnivorous Reducing Meat; VD, Vegetarian Diet; VG, Vegan Diet. Coded turns, excluded the ones from the researchers and the inaudible or neutral. The bold values in column 5 represent the highest category in each episode.

adequate for a whole society; advanced by Bea as early as in turn 24, was then backed by Blas. The second in frequency, in four episodes, initiated by Breixo, argued that VG could be nutritionally adequate with the use of supplements, which was strongly opposed by Blas; then the argument evolved to the issue of whether supplements could be considered "food" or not, "natural" or not, and hence if such VG, involving supplements, should be socially promoted.

A third supporting argument was contextualized in Galiciathe autonomous region where the university is located-and it justified OD in the damages for Galician economy of a radical reduction of meat intakes. In the economy handout, part of the data set provided, there is information about the weight of breeding (66.6\%) over agriculture (28.7\%) in the livestockfarming complex in Galicia, as well as the relevance of the food industry in Galician exports. This argument was explicitly carried out in three episodes. The remaining two supporting arguments were not always explicit although they were underlying a great deal of the debate: the relevance accorded to nutrition over other dimensions in opposition to ethical concerns, and the cultural weight of OD and eating meat, in contrast with Breixo's insistence in requiring evidence for OR. Figure 1 represents the six arguments.

The transition from proposing OD to OR, in episode four, may illustrate how several argumentative lines and emotive moves are intertwined in the discourse of participants:

108 Breixo: You, which diet... which diet seems more adequate? $\quad$ L Which one would you defend?

109 Bea: Huh... At first glance I would defend the omnivorous M one.

110 Breixo: Omnivorous. You? [to Blas] L

111 Bea: [interrupting] But, wait, I would like to add...

112 Blas: Huh, omnivorous but with higher vegetarian presence, a L

lacto-vegetarian or something... a very occasional meat consumption...

113 Bea: [at the same time] Yes, sure, perhaps with higher meat L reduction 


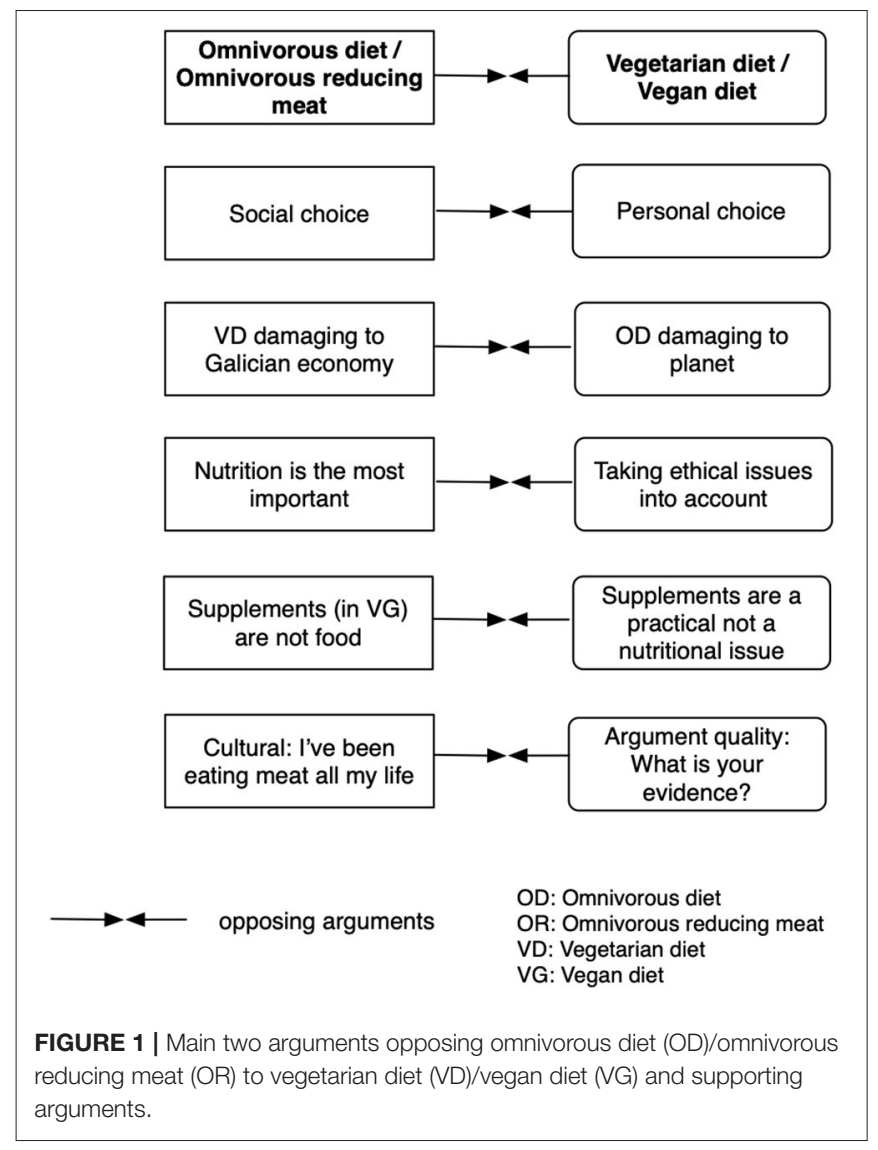

114 Breixo: But, look, then it is omnivorous... look, lacto-vegetarian is not omnivorous.

115 Blas: Huh... okay, no, but...

116 Breixo: No, lacto-vegetarian you do not eat meat.

117 Bea: But with meat reduction.

118 Blas: [interrupting] No, you eat [sic] milk or... reducing meat, $\quad H$

omnivorous diet, yes.

119 Breixo: Sure, what I think is...

120 Bea: With a... through an ethical and rational thinking and

perhaps reducing the amount of meat in diets...

121 Breixo: But then... but then that is omnivorous.

122 Bea: Yes.

123 Breixo: You [to Blas] are not saying omnivorous.

124 Blas: No, but neither am I completely saying, I am not

saying... for instance, veganism seems aberrant to me. under the term "lacto-vegetarian." This originated an exchange about its meaning with Breixo, who appealed to the criterion of the presence or absence of meat. Furthermore, Bea (120) supported OR with another rhetorical move qualifying meat reduction as "ethical and rational thinking," which is interpreted as introducing emotive tones. We distinguish these nominal appeals to ethics from actual debates about the ethical dimension, in terms of animals' well-being, which will be addressed later. As a summary, Bea and Blas central argument "OD is the more adequate" was supported in two ways: first, using the modified claim "omnivorous reducing the amount of meat"-which would implicitly address criticisms to health risks and environmental damage derived from a regular OD-later even labeled as "lactovegetarian"; and second, in a generic appeal to ethical thinking. In episode 4, low tension (L) predominates as it happens in six out of the first eight episodes.

A repeated supporting argument justified OD on the basis that VD would not be socially adequate. It was first advanced by Bea (26) in episode one, appealing to the impact of VD on Galician economy, coded as M, place closeness, and its relevance was acknowledged by Breixo.

26 Bea: Sure, because for instance... here [handout] it tells about Galician economy, does it? About diets huh... what happens? That for instance reading about ecology, vegetarian is better. I mean, it is the one less harming for the planet, that is what they say in this document. But if everybody would choose the vegetarian option, it would affect Galicia negatively. Then, it is not the same that we would decide a diet for all or personally.

Bea's argument had a double-edged claim that exemplifies the conflicting nature of SSI, which is a scientific question with social consequences. Rather than an explicit defense of OD, the claim was a criticism to VD, justified in the negative effects for Galician economy if VD becomes widely adopted; despite acknowledging that VD is better for the environment. This emotive framing has a strong place-closeness component. In episode three, the orientation changed from damage to economy toward the social impossibility of taking supplements: Bea's utterance (95) is coded as $\mathrm{H}$, a rhetorical question:

95 Bea: Would you [to Breixo], for society, promote a diet that would require supplements? Rather than a balanced diet that would have food...

Although in episodes one and three there is a higher frequency of L utterances, the issue of supplements resurfaced again in episode seven in a heightened tone; in that episode, $\mathrm{L}$ and $\mathrm{M}$ are tied with nine turns each, and there are seven $\mathrm{H}$ turns:

224 Bea: Huh... I believe that if you are going to recommend $\mathrm{M}$ something, I mean, if it were a personal decision I wouldn't mind, in fact I sometimes take supplements, although I am not vegetarian, but...

225 Breixo [interrupting] But, I mean, implicitly... $\mathrm{H}$

226 Bea:...to promote it as something social, I wouldn't promote something that would need complements to be...

227 Breixo: Fine, but why? Because implicitly you are recognizing that it is a pain in the ass to deal with supplements... or why? 228 Bea: But not because it is a pain in the ass, but because the diet that I am recommending has deficits and needs from other 
things, from, er... the chemist or as the pharmacy or...

(...)

235 Blas: [interrupting] Imagine, to tell people that from now on.. imagine we are the ministry and we were in a banana republic... from those that we could impose what people eat, wouldn't be? Sure, to tell people that we are going to have a diet with... that they need to go to the pharmacy to buy supplements... it seems to me something...

After the debate about supplements, the focus of the socialpersonal dilemma shifted to the need for them, in a move overlapping VGs-which need supplements-with VDs-which do not. Breixo (227) criticized the justification, which he considered of a practical nature, not nutritional. Blas (235) heightened the emotional tension: first, comparing hypothetical recommendations to impositions of a banana republic and second, opposing food to products bought in pharmacies. It should be noted that the improbability of a mass adoption of VD was not discussed.

\section{Emotional Tension as a Process of Framing} In the participants' discourse, fluctuations in emotional tension interacted with the evaluation of evidence across a range of dimensions. We focus primarily on the building of a frame that oriented the consensus and the conclusions stated in their final essay, and secondarily on the use of themes carrying affective weight.

The emotive building of a frame through an interaction between emotional tension and evidence may be illustrated by the appeals to ethical issues. It represents a conflict between two norms that are difficult to reconcile: the consideration of meat as the standard diet, and ethical principles that would establish the undesirability of mistreating or killing animals. Ethics were first invoked by Bea in episode four, turn 120 (reproduced above), when she proposes a reduction in meat consumption. She further develops this issue later in the same episode:

144 Bea: I am saying something varied, [eating] a little bit of

$\mathrm{H}$

everything. But with... with rationality, I mean without

slaughterhouses that cause... excessive suffering to animals, to

value the... the ethical dimension... a little bit of everything with rationality.

145 Blas... [interrupting] And intensive fisheries exploitation...

146 Bea: I... I mean, I don't know whether the best option would

$\mathrm{H}$ be the vegetarian one, but to me... in my view... fuck, l've been eating meat all my life and... I believe that if you do it with rationality and responsibility, it could be a good option for society [rising intonation on "society"].

Bea and Blas' choice of OR was implicitly based on the evidence about environmental and nutritional impact of OD discussed in episodes 1-3. These pieces of evidence, as the one mentioned by Blas, are modulated by the interaction with values and emotions, such as the ethical dimension (Bea, 144), with a specific reference to animal suffering and to slaughterhouses, which carry an unpleasant emotive weight. She further continued (146) by laying out her argument: the choice of OR is based on the supporting argument "good option for society." The justifications for this second claim were explicitly of cultural and emotional nature "I've been eating meat all my life," and the claim is reinforced by the curse, the rising intonation, and implicitly by the anticipated impact of VD to Galician economy. All these utterances are coded as high tension $(\mathrm{H})$. The intertwining of emotions and evidence in her argument are represented in Figure 2.

The conflict between two sets of values, ethical and culturalemotional, is carried out to the last episodes, 8-10, when emotional tension reached its peak:

250 Blas: Well, ethical dimension, which is ours... we talk about M Peter Singer [...] from two or several ways of feeding ourselves we should choose the one causing less harm, shouldn't we? Which is...

251 Breixo: Therefore the vegan one, right?

252 Blas: Sure, here it would opt for vegan ones, but huh... but we cannot open an ethical reason at the expense of one... of a nutritional argument.

253 Breixo: How?

256 Blas: Then we need to attend to nutrition, which is important. Sure, it is, why do we eat? Why do we have a diet? Why do we feed ourselves? In order to... gain something, whatever they are. Then, in this sense, I mean, we should contemplate ethics, but with priority to...

259 Bea:... it [omnivorous diet] would be missing a more ethical use that the one it has today and... I mean, it is not the most perfect option from an ethical perspective, but...

261 Bea: Sure, because to me is much more ethical to kill [lowers her voice] an animal in order to eat it and to use its skin for clothes than to kill it only to... to take leather, or things [...] I believe that it is truly more ethical if you sacrifice an animal, make the most of its use completely...

267 Blas: Yes, yes, we have there a problem of ethics against nutrition

In this excerpt from episode 8, Blas explicitly acknowledged the conflict between nutrition and ethics, claiming that they should give priority to nutrition, with a rhetorical question (256). Bea, in her efforts to build a supporting argument for an OR, continued to develop notions about more or less "ethical" ways of killing animals; it may be noted that she lowers her voice when saying "to kill" (261), arguably ashamed of acknowledging that eating implies killing.

In episode nine the debate was being framed in a life-death opposition, after Breixo challenged the other three members, who defended OR, to justify in which way that option would satisfy the ethical criteria for adequately treating animals:

291 Breixo: Ok, ok, but ethically...? Why... why a reduced M omnivorous diet? Because you are still eating animals.

292 Blas: Because... man, I eat some animals, but... you can't die yourself to save the animals, mate. That's... phew!

293 Breixo: But who is talking about dying...

294 [they speak simultaneously, inaudible]

295 Breixo: But it has been proved that you won't die if you don't eat animals. I mean, there are vegan people out there in the world. 


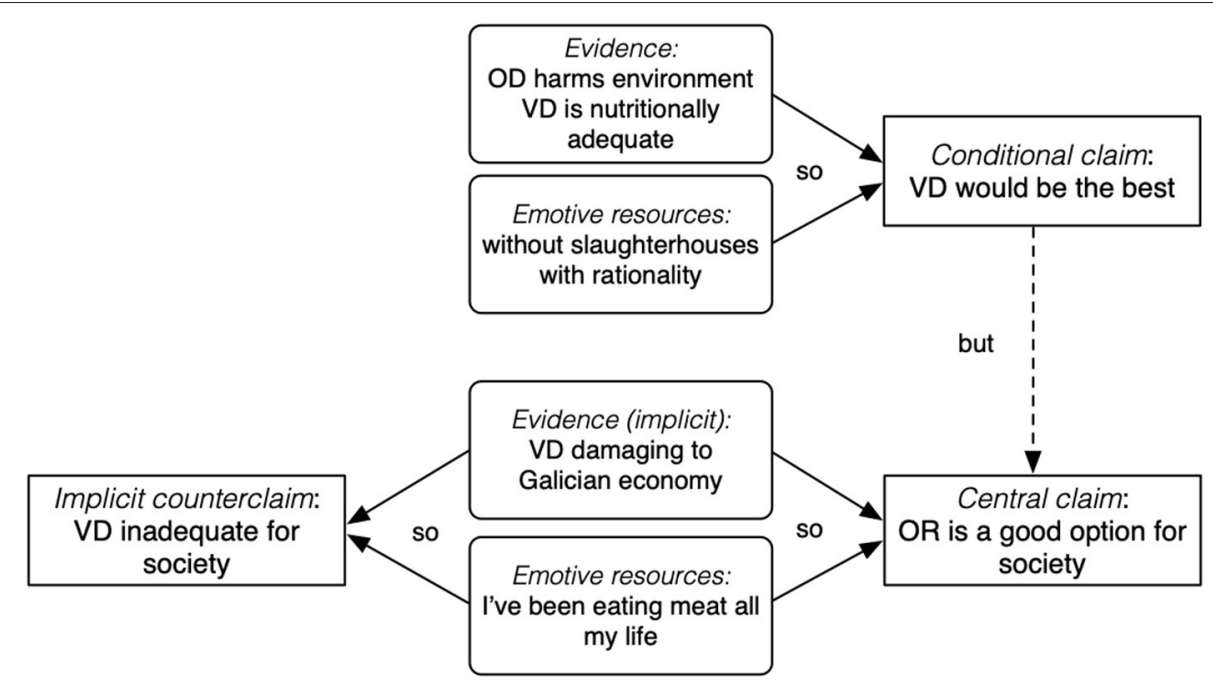

OD: Omnivorous diet VD: Vegetarian diet

OR: Omnivorous reducing meat

VG: Vegan diet

FIGURE 2 | Bea's argument in episode four, combining evidence and emotive resources.

296 Blas: An underfed human species, right? I want to belong to a hypertrophied human species, at least to a certain extent, right? [laughs]

297 Breixo: Ok, but come on, we have to argument properly.
347 Breixo: I can tell you that breeding a rabbit... breeding rabbits to eat them goes against the basic interest of any animal, which is to carry on with living.
Blas (292) claim set the issue as death either for humans or for non-human animals, framing it in a high emotional tone and driving the focus away from the ethical implications. He was implying that killing animals (in order to eat them) is unpleasant, but dying oneself is even worse.

The emotional tension keeps rising in the last episode, the 10th, in which 12 out of 17 turns are coded as H. Breixo accepted the OR option in order to build a consensus, although he still strived for developing an evidence-based argument, trying to meet quality criteria.

338 Breixo: I... can accept it [your position]. But then I still don't... I still don't know what is leading you to make a reduction of meat consumption.

339 Bea: Okay, because of ethical reasons... So there is not...

340 Breixo: Ethical reasons, what? Because at the end the

animal... you raise it to be... [he omits wat would probably be "killed"]

341 Bea: Well, but one thing is uh... to have a control of how this killing is and... all this process, and another thing is to do it massively as...

342 Breixo: And why is this more ethical?

343 Bea: Fuck!

345 Blas: Man, it has always been more ethical going to hunt a rabbit in the wild than keeping twenty thousand rabbits there locked up, all dejected, isn't it?

Bea and Blas, in their defense of the OD, made attempts to present the issue as a question of "killing animals ethically." It may be interpreted as a way of avoiding the causal (unpleasant) implication of eating animals: in order to eat them, you have to kill them first.

In the last three episodes, particularly in the 10th, the argumentative exchanges are framed in a life-death emotive positioning. We interpret that, in this context, the emotional framing takes over the evaluation of evidence, which up to that point had been used by Breixo to support the vegetarian (or even vegan) options. Figure 3 represents the opposition between the two sides of the central argument across the 10 episodes, and how emotive resources are employed in building a frame that oriented the debate toward consensus-although, as discussed later, it was not an actual consensus, but sort of a forced one, arguably because of time constraints-and hence toward the decision stated in their final essay.

It may be noted that, as represented in Figure 3, participants appealed to emotive resources as soon as in the first episode: for instance, the negative (unpleasant) potential impact of a large-scale adoption of VDs for Galician economy, where cattle breeding has a greater weight than agriculture. This emotive positioning, related to a strong place closeness, resurfaced later in other episodes. 

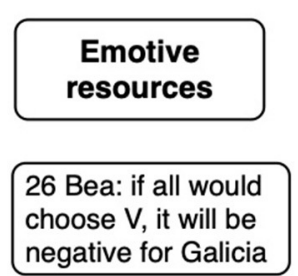

$74 / 76$ Blas: is VG good? Damnit! / Is not home cooking

95 Bea: Would you?

124 BI: VG is aberrant
144 Bea: without
slaughterhouses
146 Bea: Fuck!

$152 \mathrm{BI}$ : responsible consumption of meat 177 Bea: I am adrift

180 BI Here in Galicia
205 Bea: controlling
slaughterhouses

227 Bre: supplements are a pain in the ass $235 \mathrm{Bl}$ : If we were a Banana republic

261 Bea: it is much more ethical to kill an animal in order to eat it

$296 \mathrm{Bl}$ ( (I don't want) to be an underfed human species

352 Bea: A responsible use, so its death is not because you like a steak ... more ethical

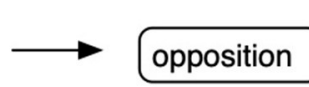

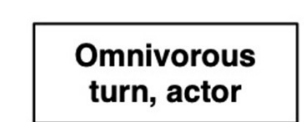
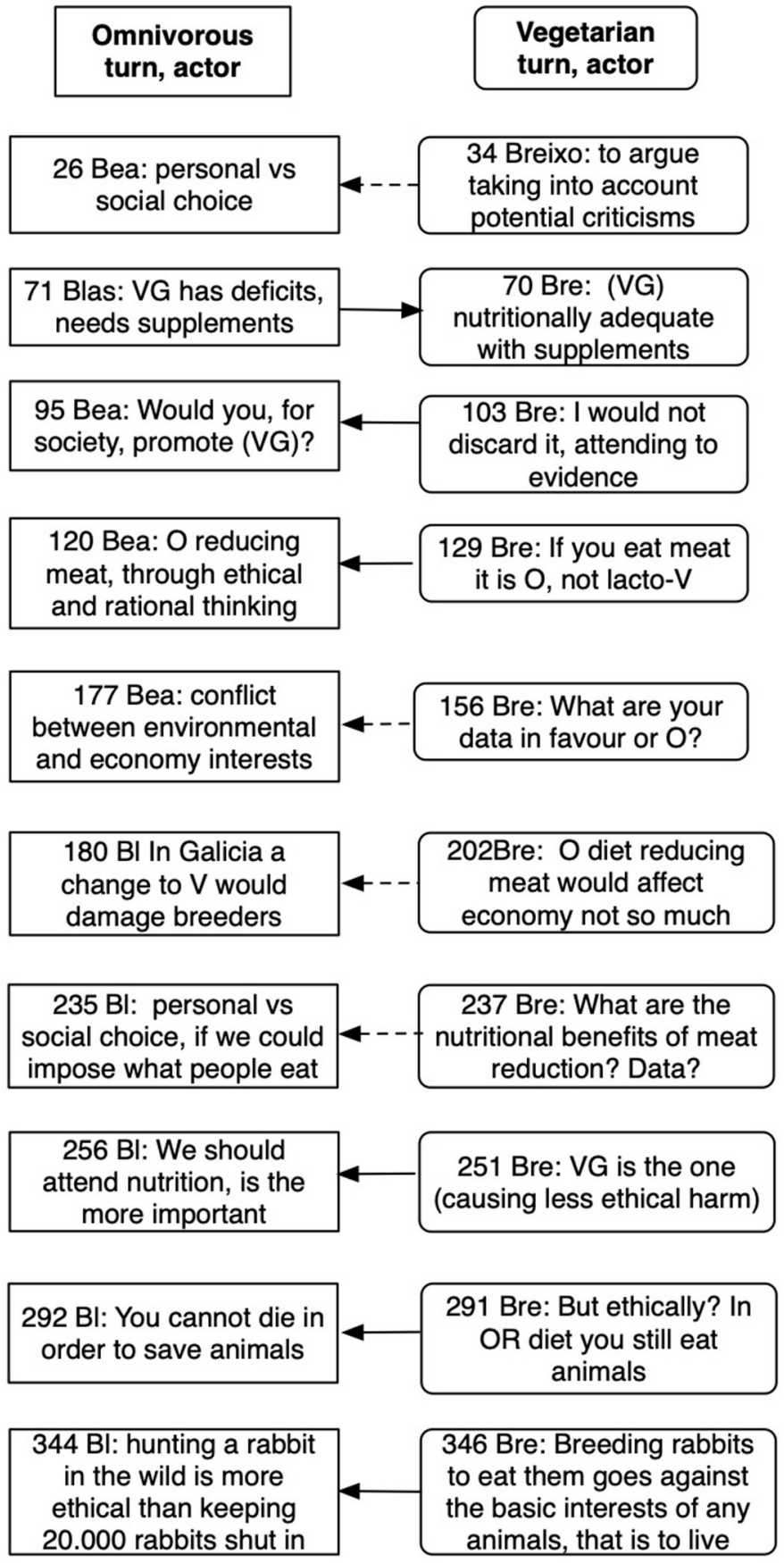

\section{O: Omnivorous diet}

OR: Omnivorous reducing meat

V: Vegetarian diet

VG: Vegan diet

FIGURE 3 | Emotive resources used in building an emotive frame through the 10 argumentative episodes opposing OD/OR to VD/VG diets. 


\section{How the Emotive Framing Oriented the Decision}

As a summary of the process of negotiating a decision, the debate showed that the initial state was one of discursive opposition between OD and VD, and that the group's strategy was exploratory, collaborating, although with uneven participation, and focusing on the epistemic conflict between ideas, not on personal oppositions. However, contrarily to other small groups (Jiménez-Aleixandre and Brocos, 2017), we interpret that they did not reach a consensus, as displayed in the final turns of the last episode reproduced above. This was one of the questions posed in the interview: were disagreements resolved and how? To this, their responses differed:

Blas: Yes [they were solved]... by time pressure. Because it was necessary to produce a work, this facilitated reaching a consensus.

Bea: I don't remember [the disagreements]... It was difficult that Breixo would accept some of the ideas.

Breixo: No, they were not resolved... we didn't make ourselves understood to the others.

Furthermore, to the question about how was the decision reached, Breixo stated that it was necessary to deliver the work on time, adding:

Breixo: It has not only arguments, but also feelings and prejudices.

We interpret these responses in the light of the debates and the final decision about OR: from the two participants proposing OR, Bea stated that she did not remember the disagreements (even though she had the opportunity of reading the transcription), and Blas saw them solved and consensus reached, probably because the decision corresponded to his proposal. On the other hand, Breixo acknowledged that the differences had not been solved, in other words, that they wrote the essay without actually agreeing about the decision, even though he accepted to bring the debate to an end. Blas and Breixo pointed to time pressure $-90 \mathrm{~min}$ of debate and previous work about the data set-and to the need for delivering an essay. Interestingly, Breixo added that the decision had been not only a matter of arguments, but also of "feelings and prejudices." We may also note that in the interview the three student teachers identified the ethical dimension as the most relevant for them, something that is not clearly reflected in the debate, or in their decision.

We reproduce the two initial paragraphs, and also the last one, from their 1,000 word essay, which was submitted as their final decision resulting from the debate (emphasis in the original):

"In our group we agreed on a diet that we consider adequate to a person under normal health conditions, and that could even be promoted for the Galician population.

We propose a low meat consumption diet; an omnivorous diet that includes not only vegetables, but also small percentages of animal meat, selected in accordance with the criteria of a responsible consumption which seeks to reduce unnecessary animal suffering, ecological sustainability, to preserve economy, and to respect our own culture." (the four paragraphs discussing, respectively, the ethical, nutritional, environmental, and economic dimensions are not reproduced)

"We finally took into account the cultural dimension of the diet. We believe that this dimension should not be overlooked; we all are born and we live within a cultural context that conditions almost every of our day-to-day practices. (...) meat consumption plays an important role in the 'traditional' Galician diet; therefore a proposal of change toward a meatfree diet would be very difficult since it would entail an important loss of a consolidated symbolic expression, which is also a part of our heritage. On the other hand, it would be feasible to propose a reduction of meat consumption, without drastically altering its cultural relevance."

Emotions were mobilized in the written arguments, which show that they are not specific of oral contexts, although the emotional tension was toned down in comparison with the oral debates. Their final decision of an OD with low meat consumption was also the most frequent in the class: 10 out of the 20 small groups chose it in their essays. In this group, we interpret that the emotional tension framed the debate, which was essential in orienting the decision toward a diet that would be emotionally acceptable for all participants as discussed below.

\section{DISCUSSION AND CONCLUSIONS}

This study examines how emotional tension acts as a process of framing in an argumentative debate about diets, and how this emotive framing drives the orientation toward the decision of an OD with meat reduction.

First, about the productivity of the argumentative process in comparison with the previous studies, the findings indicate that participants attended to the epistemic dimension of the dilemma, systematically discussing pieces of evidence related to each of the five dimensions involved: environmental impact of diets (episodes 1 and 5), nutrition (episodes 2-4), economy (episode 6), ethical issues involved in diets (episodes 7-10), and cultural dimensions (across several episodes). They did so while sustaining an exploratory discourse without interpersonal conflicts. Thus, they achieved, to a certain extent, productive discourse, avoiding the pitfalls pointed out by Asterhan (2013), of either excessive confrontation or lack of critical discussion. The participants in our study, like the ones in Isohätälä et al. (2018), maintained favorable socio-emotional processes. However, while Isohätälä et al. (2018) found that they mostly failed to engage in argumentation, the participants in our study did engage in it, although their evidence evaluation was not carried up to their final decision in a fully coherent way. We interpret that, while participants made extensive use of emotive resources, these were oriented toward the discourse objects (Grize, 1996), in other words, toward the diets, rather than toward other participants: they never had a personal target, which contributed to sustain a favorable affective climate. Cultural background may have played a role in a different way in which Galician (Spanish) student teachers' engaged in argumentation, in comparison with their Finnish counterparts in Isohätälä et al. (2018) study. 
This issue would need to be further explored in international comparative research.

Second, appeals to emotions and to evidence were deeply intertwined in the arguments of participants. This is reflected in the findings in several ways, for instance, in the range of themes employed in the emotive construction of the discourse, as summarized in Supplementary Table 1; particularly in the positioning axis. Participants framed the debate about diets on a life-death opposition, and developed meanings for "ethical" ways of eating, breeding, and even of killing. As Hufnagel (2015, 2019) has shown, emotional sense-making and meaning-making are related to the use of emotions as evaluative mechanisms, and this use points to personal relevance and deep relationships to ideas or objects (of discourse, we would add). A second instance is the content of the supporting arguments, represented in Figure 1, as, for example, the focus on social choices, the anticipated damages of VD to Galician economy, which has strong closeness for participants; or the cultural weight of family, and of traditional diets. A third instance are the arguments reproduced in the excerpts, revealing a combination of appeals to evidence and of emotional tension. Our results indicate that the construction of an argument and the construction of an emotive position are deeply connected, a finding coherent with Polo et al.'s (2013) findings.

Third, the findings suggest that arguments' quality, according to structural criteria, can be compatible with the integration of emotive resources. For instance, the written essay of the group is an example of integration of several lines of reasoning, articulating evidence, and values (Jiménez-Aleixandre and Brocos, 2017). Bea's argument in episode 4, represented in Figure 2, is a valuable argument from a structural point of view. However, in that argument, as in other cases, emotional tension is used to "reduce" the weight of the ethical considerations about ODs, be it OD or OR.

Fourth, the findings are coherent with our previous proposal (Jiménez-Aleixandre and Brocos, 2018) about the differences between discursive contexts of argumentation, in particular about the focus on acceptability in decision-making contexts, which is different from plausibility in the evaluation of causal explanations. For instance, while in arguments about potential explanations for the yellow color of farm chickens (JiménezAleixandre et al., 2000), the students evaluate if it is plausible that the cause is heredity or eating yellow feed, in this study the debate is about the acceptability of VDs, particularly for its social implementation.

Finally, the analysis shows that the emotional tension built by two participants, Bea and Blas, was successful in achieving an emotive framing, which influenced the dynamics of argument construction in the group. In the central argument OD vs. VD, the benefits-attested by the pieces of evidence-of VD were weighed against what was implicitly perceived as threatening for lifestyles. Thus, in their essay, both in the first and last paragraphs, they focus on the feasibility of promoting a diet that would be acceptable for the Galician society, without challenging "a consolidated symbolic expression, which is also a part of our heritage." In the diets' dilemma, more dimensions were emotively framed as negative (unpleasant) than as positive (pleasant), which arguably had consequences for the final decision.

We suggest that the analysis in terms of emotive framing, which is an original contribution of our study, may be a fruitful approach for sophisticated studies of argumentation about SSI. Future lines that we plan to explore through fine-grained analysis of the contribution of emotive resources are the relationships between the mobilization of emotions, emotional tension, and the participants' perceptions of their own agency. Educational implications are, for instance, the interest of designing argumentation tasks that specifically take into account this emotive dimension, which may lead to a deeper student engagement and personal agency. This is relevant for SSI and in particular for the question of sustainable diets as it depends largely on personal decisions rather than on institutional responsibility. Our findings suggest that reasoning is not performed in a neutral space, but rather is emotionally and personally motivated. On one hand, this could make more difficult the epistemic construction of arguments, but on the other hand, emotional implication could be an asset for engaging in urgent issues, as environment deterioration and climate change. As Brown and Stenner (2001) pointed out, discussing Spinoza, "Cartesianism, in setting us against nature, sets us against ourselves" (p. 87), a sentence that we read as an anticipation of the One Health concept-human health is inseparable from animal and plant's health. There is a need for humans to grasp these connections and set us for nature, rather than against it.

\section{DATA AVAILABILITY STATEMENT}

The raw data supporting the conclusions of this article will be made available by the authors, without undue reservation.

\section{ETHICS STATEMENT}

Ethical review and approval was not required for the study on human participants in accordance with the local legislation and institutional requirements. The patients/participants provided their written informed consent to participate in this study.

\section{AUTHOR CONTRIBUTIONS}

All authors listed have made a substantial, direct and intellectual contribution to the work, and approved it for publication.

\section{FUNDING}

This work was supported by the Spanish Ministry of Science, Education and Universities and is partly funded by the European Regional Development Fund (ERDF). Contract grant PGC2018096581-B-C22.

\section{SUPPLEMENTARY MATERIAL}

The Supplementary Material for this article can be found online at: https://www.frontiersin.org/articles/10.3389/fpsyg. 2021.662141/full\#supplementary-material 


\section{REFERENCES}

Andriessen, J., Baker, M., and van der Puil, C. (2011). "Socio-cognitive tension in collaborative working relations," in Learning Across Sites: New Tools, Infrastructures and Practices, eds S. Ludvigsen, A. Lund, I. Rasmussen and R. Saljo (London: Routledge), 222-242.

Asterhan, C. S. C. (2013). "Epistemic and interpersonal dimension of peer argumentation: conceptualization and quantitative assessment," in Affective Learning Together. Social and Emotional Dimensions of Collaborative Learning, eds M. Baker, J. Andriessen, and S. Järvelä (Milton Park: Routledge), 251-271.

Avraamidou, L. (2020). Science identity as a landscape of becoming: rethinking recognition and emotions through an intersectionality lens. Cult. Studies Sci. Educ. 15, 323-345. doi: 10.1007/s11422-019-09954-7

Baker, M., Andriessen, J., and Järvelä, S. (Eds.). (2013a). Affective Learning Together. Social and Emotional Dimensions of Collaborative Learning. Milton Park: Routledge. doi: 10.4324/9780203069684

Baker, M., Andriessen, J., and Järvelä, S. (Eds.). (2013b). "Introduction: visions of learning together," in Affective Learning Together. Social and Emotional Dimensions of Collaborative Learning, eds M. Baker, J. Andriessen, and S. Järvelä (Milton Park: Routledge), 1-30.

Berland, L. K., and McNeill, K. L. (2010). A learning progression for scientific argumentation: understanding student work and designing supportive instructional contexts. Sci. Educ. 94, 765-793. doi: 10.1002/sce.20402

Bravo-Torija, B., and Jiménez-Aleixandre, M. P. (2018). Developing an initial learning progression for the use of evidence in decision-making contexts. Int. J. Sci. Math. Educ. 16, 619-638. doi: 10.1007/s10763-017-9803-9

Brocos, P., and Jiménez-Aleixandre, M. P. (2020). "What to eat here and now: Contextualization of scientific argumentation from a placebased perspective," in International Perspectives on the Contextualization of Science Education, ed I. Sánchez-Tapia (Dordrecht: Springer), 15-46. doi: 10.1007/978-3-030-27982-0_2

Brown, S. D., and Stenner, P. (2001). Being affected: Spinoza and the psychology of emotion. Int. J. Group Tensions. 30, 81-105. doi: 10.1023/A:1026658201222

Creswell, J. W. (2013). Qualitative Inquiry \& Research Design (third edition). Thousand Oaks, CA: Sage Publications.

Cui, J., Li, F., and Shi, Z.-L. (2019). Origin and evolution of pathogenic coronaviruses. Nat. Rev. Microb. 17, 181-192. doi: 10.1038/s41579-018-0118-9

Darner, R. (2019). How can educators confront science denial? Educ. Res. 48, 229-238. doi: 10.3102/0013189X19849415

Denzin, N. K., and Lincoln, Y. S. (2013). Collecting and Interpreting Qualitative Materials (fourth edition). Thousand Oaks, CA: Sage Publications.

Evagorou, M., and Puig, B. (2017). Engaging elementary school pre-service teachers in modeling a socioscientific issue as a way to help them appreciate the social aspects of science. Int. J. Educ. Math. Sci. Techn. 5, 5113-123. doi: $10.18404 /$ ijemst.99074

Food and Agriculture Organization of the United Nations (FAO) (2009). How to Feed the World in 2050. Rome: High-Level Expert Forum.

Gee, J. P. (2014). An Introduction to Discourse Analysis: Theory and Method. New York, NY: Routledge. doi: 10.4324/9781315819679

Glaser, B. G., and Strauss, A. L. (1967). The Discovery of Grounded Theory: Strategies for Qualitative Research. London: Aldine de Gruyter.

Grize, J.-B. (1996). Logique Naturelle et Communication (Natural Logic and Communication). Paris: PUF. doi: 10.3917/puf.grize.1996.01

Herman, T., and Serafis, D. (2019). Emotions, argumentation and argumentativity: insights from an analysis of newspapers headlines in the context of the Greek crisis. Informal Logic 39, 373-400.

Hufnagel, E. (2015). Preservice elementary teachers' emotional connections and disconnections to climate change in a science course. J. Res. Sci. Teach. 52, 1296-1324. doi: 10.1002/tea.21245

Hufnagel, E. (2019). "Emotional discourse as constructed in an environmental science course," in Theory and Methods for Sociocultural Research in Science and Engineering Education, eds G. J. Kelly and J. L. Green (New York, NY: Routledge), 155-180. doi: 10.4324/978135113 9922-7

Hufnagel, E. (2021). "Emotional sense-making and critical thinking in the era of post-truth: the case of climate change," in Critical Thinking in Biology and Environmental Education. Facing Challenges in a Post-Truth World, eds B. Puig and M. P. Jiménez-Aleixandre (Cham: Springer) (in press).
Hyland, J. J., Henchion, M., McCarthy, M., and McCarthy, S. (2017). The role of meat in strategies to achieve a sustainable diet lower in greenhouse gas emissions: a review. Meat Sci. 132, 189-195. doi: 10.1016/j.meatsci.2017.04.014

International Agency for Research on Cancer (2015). IARC Monographs Evaluate Consumption of Red Meat and Processed Meat. Press release $n^{\circ} 240$. Available online at: https://www.iarc.who.int/wp-content/uploads/2018/07/pr240_E.pdf

IPBES (2020). IPBES Workshop on Biodiversity and Pandemics. Executive report. Retrieved from: https://ipbes.net/pandemics

IPCC (2019). Climate Change and Land: An IPCC Special Report on Climate Change, Desertification, Land Degradation, Sustainable Land Management, Food Security, and Greenhouses Gas Fluxes in Terrestrial Ecosystems. Retrieved from: https://www.ipcc.ch/srccl/

Isohätälä, J., Näykki, P., Järvel, S., and Baker, M. J. (2018). Striking a balance: socio-emotional processes during argumentation in collaborative learning interaction. Learn. Cult. Soc. Inter. 16, 1-19. doi: 10.1016/j.lcsi.2017.09.003

Jiménez-Aleixandre, M. P., and Brocos, P. (2017). "Processes of negotiation in socio-scientific argumentation about vegetarianism in teacher education," in Interpersonal Argumentation in Educational and Professional Contexts, eds F. Arcidiacono and A. Bova (Dordrecht: Springer), 117-139. doi: 10.1007/978-3-319-59084-4_6

Jiménez-Aleixandre, M. P., and Brocos, P. (2018). "Shifts in epistemic statuses in argumentation and in conceptual change," in Converging Perspectives on Conceptual Change: Mapping an Emerging Paradigm in the Learning Sciences, eds T. Amin and O. Levrini (Oxford: Routledge), 171-179. doi: $10.4324 / 9781315467139-21$

Jiménez-Aleixandre, M. P., Brocos, P., and Puig, B. (2019). "Embedding conflict in argumentation task design in teacher education," in Invited presentation at the International Conference Innovation in Learning Instruction and Teacher Education (ILITE 1) (Hanoi, Vietnam), 14th-15th December.

Jiménez-Aleixandre, M. P., Bugallo Rodríguez, A., and Duschl, R. A. (2000). "Doing the lesson" or "doing science": argument in high school genetics. Sci. Educ. 84, 757-792. doi: 10.1002/1098-237X(200011)84:6<757::AID-SCE $5>3.0 . \mathrm{CO} ; 2-\mathrm{F}$

Jiménez-Aleixandre, M. P., and Erduran, S. (2008). "Argumentation in science education: an overview," in Argumentation in Science Education: Perspectives From Classroom-Based Research, eds S. Erduran and M. P. Jiménez-Aleixandre (Dordrecht: Springer), 3-27. doi: 10.1007/978-1-4020-6670-2_1

Kelly, G. J. (2021). Theory, methods, and expressive potential of discourse studies in science education. Res. Sci. Educ. 51 225-233. doi: 10.1007/s11165-020-09984-0

Kerbracht-Orecchioni, C. (1980). L'énonciation de la subjectivité dans le langage. Paris: Armand Colin.

Kuypers, J. A. (2009). Rhetorical criticism: Perspectives in action. Lanham, MD: Lexington Books.

López-Facal, R., Jiménez-Aleixandre, M. P., and Arcidiacono, F. (2015). "Le territoire comme composante de l'identification nationale dans l'argumentation des élèves du secondaire," in Largumentation dans les contextes de l'éducation, eds N. Muller Mirza and C. Buty (Bern: Peter Lang), 323-354. doi: 10.3726/978-3-0351-0848-4

Micheli, R. (2010). Emotions as objects of argumentative constructions. Argumentation 24, 1-17. doi: 10.1007/s10503-008-9120-0

Morin, O., Simonneaux, L., Simonneaux, J., Tytler, R., and Barraza, L. (2014). Developing and using an $S^{3} R$ model to analyze reasoning in webbased cross-national exchanges on sustainability. Sci. Educ. 98, 517-542. doi: $10.1002 /$ sce. 21113

Muller Mirza, N. (2013). "A sociocultural perspective on conflict in argumentative design," in Affective Learning Together. Social and Emotional Dimensions of Collaborative Learning, eds M. Baker, J. Andriessen, and S. Järvelä (Milton Park: Routledge), 233-250.

Osborne, J., Henderson, J. B., MacPherson, A., Szu, E., Wild, A., and Yao, S.Y. (2016). The development and validation of a learning progression for argumentation in science. J. Res. Sci. Teach. 53, 821-846. doi: 10.1002/tea.21316

Plantin, C. (2011). Les Bonnes Raisons Des Émotions. Bern: Peter Lang. doi: 10.3726/978-3-0352-0070-6

Plantin, C. (2019). Tense arguments: questions, exclamations, emotions. Informal Logic. 39, 347-371. doi: 10.22329/il.v39i4.6043

Pollaroli, C., Greco, S., Oswald, S., Miecznikowski-Fuenfshilling, J., and Rocci, A. (2019). Preface. Informal Logic. 39, 287-300. doi: 10.22329/il.v39i4.6047 
Polo, C. (2014). L’eau à la bouche: ressources et travail argumentatif des élèves lors de débats socio-scientifique sur l'eau potable (Unpublished doctoral dissertation). Université Lumière, Lyon 2.

Polo, C., Plantin, C., Lund, K., and Niccolai, G. (2013). Quand construire une position émotionnelle, c'est choisir une conclusion argumentative: le cas d'un café-débat sur l'eau potable au Mexique. Semen 1, 41-63. doi: $10.4000 /$ semen. 9800

Polo, C., Plantin, C., Lund, K., and Niccolai, G. (2017). Group emotions in collective reasoning: a Model. Argumentation 31, 301-329. doi: 10.1007/s10503-016-9407-5

Richter, T., and Maier, J. (2017). Comprehension of multiple documents with conflicting information: a two-step model of validation. Educ. Psych. 52, 148-166. doi: 10.1080/00461520.2017.1322968

Schwarz, B. B., and Goldberg, T. (2013). "Look who's talking': Identity and emotions as resources to historical peer reasoning," in Affective Learning Together. Social and Emotional Dimensions of Collaborative Learning, eds M. Baker, J. Andriessen, and S. Järvelä (Milton Park: Routledge), 272-292.

Spinoza, B. (1677/2012). Ética. Barcelona: Ediciones de Intervención Cultural.

Stehfest, E., Bouwman, L., van Vuuren, D. P., den Elchen, M. G. J., Eickhout, B., and Kabat, P. (2009). Climate benefits of changing diet. Clim. Change 95, 83-102. doi: 10.1007/s10584-008-9534-6

Thompson, S., Gower, R., Darmon, N., Vieux, F., Murphy-Bokern, D., and y Maillot, M. (2013). A Balance of Healthy and Sustainable Food Choices for France, Spain, and Sweden. Surrey: World Wildlife Fund.
Tilman, D., and Clark, M. (2014). Global diets link environmental sustainability and human health. Nature 515, 518-522. doi: 10.1038/nature 13959

Toulmin, S. (1958). The Uses of Argument. Cambridge: Cambridge University Press.

Voloshinov, V. N. (1986). Marxism and the Philosophy of Language. Cambridge, MA: Harvard University Press.

Zeidler, D. L., and Sadler, T. D. (2008). "The role of moral reasoning on argumentation: conscience, character and care," in Argumentation in Science Education: Perspectives From Classroom-Based Research, eds S. Erduran and M. P. Jiménez-Aleixandre (Dordrecht: Springer), 201-216. doi: 10.1007/978-1-4020-6670-2_10

Conflict of Interest: The authors declare that the research was conducted in the absence of any commercial or financial relationships that could be construed as a potential conflict of interest.

Copyright $\odot 2021$ Jiménez-Aleixandre and Brocos. This is an open-access article distributed under the terms of the Creative Commons Attribution License (CC BY). The use, distribution or reproduction in other forums is permitted, provided the original author(s) and the copyright owner(s) are credited and that the original publication in this journal is cited, in accordance with accepted academic practice. No use, distribution or reproduction is permitted which does not comply with these terms. 


\section{OPEN ACCESS}

Edited by:

Isabel Menezes,

University of Porto, Portugal

Reviewed by:

Wiel Veugelers,

University of Humanistic Studies,

Netherlands

Pedro Daniel Ferreira,

University of Porto, Portugal

${ }^{*}$ Correspondence:

Antonia Larrain

alarrain@uahurtado.cl

Specialty section: This article was submitted to Educational Psychology, a section of the journal

Frontiers in Psychology

Received: 01 February 2021 Accepted: 18 May 2021

Published: 21 June 2021

Citation:

Larrain A, Fortes $G$ and Rojas MT (2021) Deliberative

Teaching as an Emergent Field: The Challenge of Articulating Diverse Research Agendas to Promote Educational Experiences for Citizenship.

Front. Psychol. 12:660825. doi: 10.3389/fpsyg.2021.660825

\section{Deliberative Teaching as an Emergent Field: The Challenge of Articulating Diverse Research Agendas to Promote Educational Experiences for Citizenship}

\author{
Antonia Larrain $^{1 *}$, Gabriel Fortes ${ }^{1}$ and Maria Teresa Rojas ${ }^{2}$ \\ ${ }^{1}$ Facultad de Psicología, Universidad Alberto Hurtado, Santiago, Chile, ${ }^{2}$ Facultad de Educación, Universidad Alberto \\ Hurtado, Santiago, Chile
}

Democracies are increasingly dependent upon sustainable citizenship, that is, active participation and engagement with the exercising of rights in a field of plural interests, often contradictory and in conflict. This type of citizenship requires not only social inclusion, habits of knowledge, and evidence-based reasoning but also argumentation skills, such as the individual and social capacity to dispute and exercise individual and social rights, and to deal peacefully with sociopolitical conflict. There is empirical evidence that educational deliberative argumentation has a lasting impact on the deep and flexible understanding of knowledge, argumentation skills, and political and citizenship education. However, these three trends of research have developed independently with insufficient synergy. Considering the relevance of deliberative education for contemporaneous democracies and citizenship, in this paper we seek to converge in a field of interlocution, calling it deliberative teaching. Our aim is to propose a way to increase the dialog and collaboration between the diffuse literature on argumentation and education, highlighting both the main theoretical and empirical gaps and challenges that remain and the possibilities to advance our knowledge and the educational impact that this integrating field could offer.

Keywords: democracy, citizenship, argumentation, deliberative teaching, social inclusion

Democracy is not an easy road to take and follow. On the contrary, it is, as far as its realization is concerned in the complex conditions of the contemporary world, a supremely difficult one.

(Dewey, 1944/1989, p. 259)

\section{INTRODUCTION}

Democracies are being challenged around the globalized world with increasing polarization, institutional crises, and undermined public trust. Although the crisis of democracy has been a topic of discussion for a while now (Merkel, 2014), one could argue that democracies have constantly been in crisis around the world. For instance, violent democracies have been described in the global South for some time, understood as democratic systems in which violence is 
intimately intertwined with, and functional to, the core of democracy, namely, elections and political participation (Von Holdt, 2014). In particular, democracy, patronage, and violence are complexly entangled to produce "low-intensity citizenships."

However, it is possible to argue that the recent questioning of the electoral process in the United States, with the consequent risk to trust in electoral institutions, and the incitement to violent riots to interrupt the electoral certification, expresses the crisis at the heart of even a minimal model of democracy (for the electoral regime, see Merkel, 2014) in one of the most developed western democracies. In addition, the inequality in civil rights may also be observed as part of a long-lasting democratic flaw, according to a mid-range notion of democracy (Merkel, 2014). Finally, social and economic inequality, although part of a maximalist notion of democracy, has proven to be a major threat to political sustainability, as demonstrated by social protests and collective and state violence in Chile in 2019.

Democracy needs not only an electoral regime and institutional guarantee of human and civil rights, or "politics" - according to Mouffe (2014) - but also "the political," that is, civic practices involved in the unfolding of power, particularly practices of dispute and dealing with conflicts. According to an agonistic notion of democracy (see DesRoches and Ruitenberg, 2018, p. 150), strong democracies, in whatever form, need citizens to be engaged in embodying their often conflicting and contradictory interests and struggling to exercise their rights to participate in and dispute decision making from different social identities and voices. Consequently, we understand the difference, tension, disagreement, and contradiction, not as a flaw of democracies but as one of their conditions: "It is only when the ineradicable character of division and antagonism is recognized that it is possible to think in a properly political manner and to face the challenge confronting democratic politics."

Moreover, democracies are increasingly challenged by specific contemporaneous forms of conflict (global crises) and social communication. New challenges, such as environmental sustainability, global pandemics and economic restrictions, and growing awareness of social, gender, ethnic, economic, and other types of injustice, are accelerated by the development and transformation of communications technology. These challenges demand urgent social action in tensioned and conflicting fields, threatening the sustainability of our ways of life, including not only environmental but also political sustainability. Therefore, the question of sustainability, in general, is central to a contemporaneous notion of democracy (Kyle, 2020), that is, whether present generations are able to deal with these kinds of global crises without risking the living conditions of future generations.

Acknowledging that democracy always involves conflict and that most of the contemporaneous conflicts we face need to be carefully dealt with to avoid risking the common way of life of future generations - points to a special notion of citizenship. We call this sustainable citizenship (Kurian et al., 2014), understanding it as active participation and engagement with the exercising of rights in a field of plural interests, often contradictory and in conflict, in a way that allows, without dissolving difference, social, cultural, political, economic, and environmental needs to be addressed. It is digital citizenship, insofar as it presupposes active, critical, and responsible engagement with digital technologies that are respectful to human rights. Sustainable citizenship, therefore, is focused on not just participation, or the experience of social conflict as a natural accompaniment to democracy: it is also focused on solving crucial and urgent problems (without dissolving conflict) in a legitimate way. This is particularly important in violent democracies in which social conflict may all too easily become violently elaborated. In these cases, a focus on sustainability in citizenship education is central, or on how to deal effectively and legitimately with pressing problems given the conflicting nature of social life. From this perspective, and especially for violent democracies, neither deliberative citizens, aspiring to solve differences and achieve social rationality, nor agonistic ones, seeking to live emotionally in social difference and plurality, are appropriate. We need something in between: citizens seeking to deal with conflicting positions and emotions in order to address urgent and pressing needs in a sustainable way.

Sustainable citizenship, understood in this way, involves the practice of articulation of a wide range of differences to define and achieve common goals, which require specific individual and social competencies but also institutional design and particular habits. Authors, such as Kurian et al. (2014), have pointed to the role that dialectical deliberation plays in sustainable citizenship, or the deliberation of key controversies as the foundation of citizenship practices, which resonates with a deliberative notion of democracy. Therefore, regardless of whether or not a deliberative democracy is realistic or desirable (see Ryfe, 2005; DesRoches and Ruitenberg, 2018), it is clear that specific individual and social competencies are needed to make our political and environmental worlds sustainable. These competencies include, but are not necessarily limited to: being engaged and willing to participate; being able to argue our points and dispute our interests; being able to understand and evaluate possible arguments; recognizing and conceding others' good arguments; being strategic in arguments and understanding others' strategies; articulating with different people; representing and legitimating others' worldviews; understanding and legitimating different types of knowledge; selecting epistemic authorities to trust in; and positioning ourselves as political and emotional actors among others. Therefore, one could argue that sustainable citizenship requires, both at a collective and individual level, political competencies, argumentation skills, content and epistemological knowledge, in addition to emotional and ethical dispositions.

Empirical evidence, however, produced through different lines of research, points to the experience of deliberative argumentation as a practice that promotes political and civic competencies (Andersson, 2015), argumentation skills (Felton et al., 2015), knowledge (Asterhan and Schwarz, 2016), and social inclusion (Aronson and Bridgeman, 1979). In other words, deliberative argumentation is a transversal key educational practice for contemporaneous citizenship, which has been recognized as such for a while (see Michaels et al., 2008). Why, if this is the case, has deliberative argumentation not been a clear educational goal worldwide? Different answers are available. First, it is a pedagogical practice that requires 
a sophisticated pedagogical design and expertise (Andriessen and Schwarz, 2009), which has not typically been considered in national curriculums or initial and in-service formation. Second, high-stakes accountability policies undermine the possibilities of schools and teachers innovating regarding argumentative pedagogical designs (Katsh-Singer et al., 2016). Third, research in the area of deliberative argumentation has been dispersed and fragmented, with insufficient synergy, drawing on different theoretical traditions and using different concepts and labels, and with limited capacity to build on one another and influence public opinion.

The aim of this paper is to outline the need to inscribe differently and, thus far, disperse research related to deliberative argumentation in education, under the same field of interlocution. We propose to consider deliberative teaching as a family of pedagogical practices, in order to visualize their contribution to multiple benefits relevant to citizenship. This does not mean that, in doing so, different approaches within this field will be homogenized; on the contrary, the recognition of common ground allows productive dispute and discussion, as well as building up one another's insights and illuminating knowledge gaps within the field, which, in turn, strengthens the possibilities to impact educational policies and agendas. The proposal does not imply a particular commitment to a deliberative view of democracy, as we have stated. The assumption on which the paper is based is that we need specific educational experiences through which the competencies needed to exercise sustainable citizenship and democratic life can be performed and, in turn, developed.

\section{DELIBERATIVE ARGUMENTATION AND SUSTAINABLE CITIZENSHIP: WHERE IS THE EVIDENCE?}

\section{Deliberative Argumentation and Argumentation Skills}

There is a line of research in developmental psychology that has focused on evaluating, through experimental design, the effect of arguing and thinking with others in educational contexts, especially among peers, on the development of argumentation skills. For instance, Kuhn et al. (1997) asked sixth-graders and young adults to discuss weekly their opinions on capital punishment with different partners in order to reach a consensus. The results showed that, although participants' opinions were highly stable, there were gains in the range and quality of the grounds of arguments that participants produced, and in their ability to consider two sides of the issue and be aware of the coexistence of multiple views. It is important to note that the aim was not for participants to persuade one another but rather to discuss their views and reach a consensus, corresponding more to a goal of reaching an agreement and understanding than winning the argument. In fact, Felton and Kuhn (2001) report analysis of dialogs showing that younger adults were less focused on undermining partners' arguments than on clarifying and elaborating upon them.
Kuhn and Crowell (2011) conducted a three-year longitudinal study, also with sixth-graders, in which students had to argue to prepare whole-class debates on social issues, first in faceto-face small groups and then through online dialogs. The results show that students significantly improved their argument quality in post-test written essays, when compared to a comparison teacher-led group. Again, students did not have to argue to win; rather, they had to develop arguments and counter-arguments and evaluate them on several occasions. Only at the end of each topic did they have to argue to win, not through persuasion but through the quality of their argumentative moves. Moreover, Crowell and Kuhn (2014) showed how a three-year intervention with sixth-graders on collaborative peer argumentation not only fostered stronger ways to counter-argue but also bridged the gap between initially low- and high-skilled students.

Kuhn and Udell (2003) reported a study with at-risk eightgraders. Through 16 lessons (12 weeks) of goal-based activities, they had to collaboratively develop reasons into an argument and then discuss the opposing side's reasons; they also had to deliberate over the best counter-arguments and rebuttals, among others, thereby preparing a debate. The results showed the effect on oral argumentation skills, particularly on the ability to formulate counter-arguments that critically address others' arguments and rebuttals, and on the quality of their individual arguments. Although the final activity was a persuasion debate, the intense argumentation activity involved a more collaborative argumentation oriented toward evaluating and deliberating the best possible counter-arguments and rebuttals.

Iordanou and Kuhn (2020) examined whether middle-school students in a 12-week intervention benefitted more from arguing in pairs (on the use of gas or solar energy) with opposing peers than they did with same-side peers. They had to construct arguments, counter-arguments, and rebuttals for both positions, and evidence regarding both positions was given. In both conditions, the task involved both face-to-face and electronic dialogs and co-constructive argumentation among same-side pairs. Under the opposing-view conditions, students' persuasive argumentation was also involved because they were instructed to convince the opposing pair that their position was superior. Finally, all of the students participated in whole-class debates. The results show the effects of opposing-view conditions on the quality of arguments and the number of counter-arguments included in post-test essays.

Reznitskaya et al. (2009) reviewed a series of studies in which students were involved in what was called collaborative reasoning. In collaborative reasoning, students deliberate together to answer controversial questions regarding key events in the literature stories, with minimal guidance from teachers. Different from the studies of Kuhn and colleagues, students did not argue in preparation for a persuading debate. The results revealed not only the appropriation of oral argumentation skills during the discussions (Anderson et al., 2001) but also the effect of oral argumentation on post-test written individual essays: students tended to include more satisfactory arguments, counter-arguments, and rebuttals than their peers in the control conditions. 
Evidence regarding computer-supported argumentation also shows that computer-assisted collaborative peer argumentation has effects on written argument construction (Nussbaum et al., 2004, 2007; Yiong-Hwee and Churchill, 2007; Bouyias and Demetriadis, 2012; Lin et al., 2012; Noroozi et al., 2013, 2016) and argumentation sequences (Jermann and Dillenbourg, 2003; Stegmann et al., 2007), in addition to quality and knowledge of argumentation (Tsovaltzi et al., 2017).

Overall, the message is consistent and has been supported by multiple qualitative studies: peer argumentation, both orally and electronically mediated, prompts students' capacity to construct arguments and counter-arguments, both to argue with others and to argue individually in written essays. The question is which kind of peer argumentation would have led to these outcomes? Although the empirical studies mentioned above involve different types of tasks, instructions, and goals, it is clear that the argumentation practices described go beyond simple persuasion, as they involve weighting in peer groups for and against arguments and deciding which are the best before persuading others. It is likely that in the studies of Kuhn and colleagues, the preparation of whole-class debates, that is, the anticipation of persuasion, may play a key role in the quality of peers deliberation (Reznitzkaya and Wilkinson, in press), but the argumentation that unfolded was deliberative rather than fully persuasive. Also, the study by Iordanou and Kuhn (2020) shows that real disagreement and discussion of opposing points of view have an additional effect.

To see whether persuasive (arguing to convince) or deliberative (arguing to reach a consensus) goals had an effect on peer dialogs, Felton et al. (2009) conducted a study with Spanish seventh-graders on sources of energy and climate change. While students in the two experimental conditions (one persuasive and one deliberative) were grouped with disagreeing peers in three sessions (one for each dilemma), students in the control condition worked individually. The results show that students in the deliberative condition outperformed students in both the disputative and control conditions. Further analysis of the dialog (Felton et al., 2015, p. 374) revealed that the deliberative goals produced more elaborative and integrative discussions than the persuasive ones, which were shorter and more closed. The authors define persuasion dialog as "an adversarial exchange in which speakers advance incompatible claims with the goal of convincing others to accept their claim"; and deliberative dialog as "a collaborative exchange in which speakers hold incompatible claims and seek to resolve these differences to arrive at a consensual decision."

In conclusion, although many unresolved questions remain, the above-mentioned evidence suggests that peer deliberative argumentation on social, literary, and socio-scientific issues prompts school-age students' argumentation skills. It develops key capacities for sustainable citizenship, namely, dealing with controversial matters committed to the quality of arguments.

\section{Deliberative Argumentation and Knowledge}

Systematic experimental evidence is produced at the crossroads between education and psychology, showing that deliberative argumentation in education promotes knowledge understanding and concept development in different curricular disciplines, including maths, science, and history. For instance, Mercer and colleagues (Mercer and Littleton, 2007), informed by sociolinguistic and sociocultural theories, conducted different quasi-experimental studies in schools and with school-age students in the United Kingdom. The results showed how an exploratory talk curriculum prompted students' knowledge in maths and science. Exploratory talk is a type of discourse described when students have to solve problems cooperatively and to engage critically but constructively with one another's ideas:

Relevant information is offered for joint consideration. Proposals may be challenged and counter-challenged, but if so reasons are given and alternatives are offered. The agreement is sought as a basis for joint decisionmaking and action. Knowledge is made publicly accountable and reasoning is visible in the talk (Mercer, 2009, p. 184).

Therefore, we argue that exploratory talk involves the deliberative use of argumentation (Felton et al., 2009), insofar as students have to solve problems collaboratively, and, in order to do so, they have to give, and challenge one another's, reasoning to reach a consensual solution.

Following a more Piagetian design, Howe and colleagues, also in the United Kingdom, conducted a series of controlled experiments to study the effect of conflict and discussion of different perspectives on conceptual understanding. For instance, they conducted an experimental study to investigate the effects of cognitive conflict, socio-cognitive conflict, and imitation on socio-legal thinking, based on students aged between 9 and 12 . The results showed that students in the experimental conditions improved significantly more than students in the control condition from pre- to post-tests. Extended modes of reasoning present in both experimental conditions - agreement with conflicting positions, and disagreements and rejections - were systematically and significantly correlated with post-test gains. Tolmie et al. (1993) conducted an experimental study of primary- and middle-school students, the aim of which was to evaluate the relationship between task design (four conditions), dialog, and conceptual understanding of "floating" and "sinking." The results revealed that the task design had a significant effect on the amount of discussion among groups, which, in turn, was strongly and statistically associated with pre- to posttest knowledge gains. The more productive condition was the one in which students were asked to agree on a prediction, test their predictions, and reach a consensus regarding the explanation for why the objects floated or sunk. The instruction to reach a consensus was, in fact, shown to be key to prompt discussion about contrary ideas and conceptual gains (Howe et al., 2000). Howe (2009) and Howe and Zachariou (2019) showed that pre- to post-test conceptual progress was related not to the group joint constructions that were appropriated and/or accepted by students but to the discussion of different points of view. 
In the United States, Michaels et al. (2008) also showed the effects of what they called accountable talk on student learning during school whole-class discussions. Accountable talk involves participants listening and engaging with one another, and extending and building on one another's contributions. Students make logical connections and are involved in reasoning, formulating, evaluating, and revising arguments and counter-arguments; they also use evidence that is publicly available. Accountable talk, therefore, as the authors state, is characterized by involving intense deliberative argumentation in classroom discussions.

In the field of argumentation and education, Larrain and colleagues, in Chile (Larrain et al., 2018, 2019, 2020), conducted a series of quasi-experimental studies in schools to evaluate the effect of peer argumentation on science learning in middleschool students. Resonating with the studies of Howe and colleagues, they also conducted the correlational analysis to account for the differential effect of dialog (argumentative moves) on learning. Students in each lesson were typically presented with a conceptual problem and asked to work in small groups to decide consensually which was the best possible solution, formulating arguments and counter-arguments. The results show that repetitive experiences of peer argumentation had an effect on conceptual learning (Larrain et al., 2018) and that frequency of individual formulation of argumentative moves, particularly counter-argumentation, predicted learning (Larrain et al., 2018, 2019, 2020).

Kaya (2013) and Aydeniz and Dogan (2016) conducted two studies with pre-service teachers in Turkey to evaluate the effect of teaching through argumentation (through small groups and classroom discussions) on the conceptual understanding of chemical equilibrium. In both cases, experimental argumentative conditions were compared with the control teacher-led - conditions. The results showed the significant effects of argumentative conditions on student learning.

In Israel, Christa Asterhan and colleagues produced pivotal laboratory-controlled evidence regarding the relation between deliberative argumentation and learning in undergraduate students. In an experimental study (Asterhan and Schwarz, 2007) on scientific conceptual change, they asked students in two groups to collaboratively solve problems on natural selection. In addition, they asked the experimental group receiving the instruction to reach a common solution through a critical in-depth discussion in which they would try to persuade one another and explain their thinking, seeking to reach the best possible solution by supporting and refuting arguments. Students in the experimental (argumentative) condition surpassed students in the control group on pre- to post-test learning gains.

In order to see whether there is a differential effect of deliberative argumentation over persuasive argumentation on learning, Asterhan and Babichenko (2015) compared a disputative style with a deliberative style of peer argumentation, manipulated via confederates. They found that students using the deliberativestyle condition outperformed those in the disputative condition on individual learning pre- to post-test gains, showing more openness to share their incomplete understandings with their partner. In a follow-up study with online discussions,
Asterhan and Hever (2015) replicated the results, discussing the previous ones by Felton et al. (2009), in which no differences between disputative and deliberative conditions regarding posttest learning were found.

Argumentation has also been conceived of as promoting epistemological knowledge relevant to citizenship; however, overall, the experimental empirical evidence supporting this relationship is scant. It is likely that this is linked to the lack of a clear theory of epistemic cognition development (Sandoval et al., 2016) and the assumption that argumentation requires a certain level of epistemological understanding (see Kuhn et al., 2000), but not the other way around.

Findings regarding content knowledge and argumentation skills have been conducted mostly in isolation. Few studies have explored the potentialities of intervention to foster both outcomes, and their relationship has mostly been unexplored. Although effects on skills, and not on knowledge, have been reported (Wecker and Fischer, 2014), there is experimental evidence that deliberative argumentation prompts knowledge and skills together (see Iordanou et al., 2019). Moreover, recently the intertwinement of knowledge and skills has been studied, with the findings showing the effect of deliberative argumentation on knowledge through skills (Larrain et al., 2020).

To summarize, although there are several knowledge gaps in the field (see Asterhan and Schwarz, 2016), there is experimental and quasi-experimental evidence (again, supported by qualitative studies not revised here), produced in different parts of the world, showing the effect of a particular type of argumentation on the conceptual understanding of social, scientific, and language issues. Although not all of the research groups view the dialog types that they study as argumentation and differences remain, we believe that they converge on the study of the effect of "deliberative argumentation" (Felton et al., 2009, 2015; Asterhan and Schwarz, 2016) with argumentation defined as an engagement in critical thinking, elaboration, and reasoning so that students "can build up a shared understanding of the issue at stake instead of merely convincing or changing their own and each other's beliefs" (Noroozi et al., 2013, p. 60). This is relevant for sustainable citizenship, which is based on the ability to articulate conflicts and differences, considering different alternatives, using the available evidence and knowledge.

\section{Deliberative Argumentation and Civic and Political Competencies}

Political competencies, understood as multidimensional inclinations, competencies, and behaviors, are key to the notion of sustainable citizenship. They involve aspects, such as political engagement, political understanding (political knowledge about theories and current political events), political skills (related to specific ways of political involvement: organizing people, political strategies, political discussion, or discourse), and political participation and democratic virtues, among others (Beaumont et al., 2006; Persson et al., 2020). The teaching of political competencies, however, has been an increasingly relevant but insufficiently investigated issue (see Beaumont et al., 2006; Andersson, 2015; Bennion and Laughlin, 2018). In the past 
15 years, however, there has been growing interest in exploring, comprehending, and evaluating the effect of political discussion as a way to develop these competencies. Beaumont et al. (2006) evaluated, through a pre-post survey design, 21 interventions that involved, among other aspects, engaged political discussions. The results show that these interventions prompted political engagement, knowledge, and skills in undergraduate students. The relationship between political discussion and knowledge was also reported in correlational studies based on surveys in adults, showing that both the frequency and level of elaboration predict knowledge (Eveland and Thomson, 2006) and that this relation is mediated by motivation and information, independent of the level of partners' information (Eveland, 2004). Moreover, there is correlational evidence based on self-reported measures showing that parent-youth political discussions predict youth political knowledge, especially when parents' political knowledge is high (McIntosh et al., 2007); adult-youth discussions predict youth civic reasoning (Alvis and Metzger, 2020); and classroom discussions predict political knowledge.

Hess and McAvoy (2014) conducted a longitudinal, mixedmethod study of high-school social studies courses (21 schools/35 teachers/1,000 students) that included the discussion and deliberation of political topics. Classroom observations, preand post-test surveys, and interviews were conducted. The results showed that classrooms in which students were involved more than $20 \%$ of the time in the discussion of controversial political issues, with significant student-to-student talk and high levels of participation, reported significantly more interest in politics as a result of taking the course. They were also more likely to enjoy the political talk and were more comfortable with disagreement. Moreover, the authors concluded that these students were more likely to develop into engaged citizens than students in other classes.

Latimer and Hempson (2012) conducted a quasi-experimental study with undergraduates aimed at evaluating deliberative polling methodology on civic engagement (among other variables) measured with pre- and post-test surveys. The results show an effect of a condition (deliberation) on civic engagement. Less straightforward evidence has also been reported: Andersson (2015) conducted an experimental field design with pre- to post-test measures and two conditions - deliberative teaching and the control as usual condition - in three upper-secondary schools in Sweden. Students were surveyed on democratic virtues (communication competence, political efficacy, and future political participation). The results show the effects of deliberative teaching in vocational programs on some democratic virtues: communication competence and political participation in male vocational programs and political efficacy in female vocational programs. In programs of ensuing academic studies, no effect was found. Persson et al. (2020) conducted a replication study in 59 classrooms (1,200 students) aimed at evaluating the effect of deliberative teaching on self-reported civic competence (political interest, knowledge, democratic values, and political discussions). No effect of the condition was found.

These contradictory results are interesting, because they point to the need not to assume but to empirically test the effect of deliberative teaching on civic competencies. However, two aspects are worth noting. First, contrary to the literature on argumentation skills and knowledge, the effect of deliberative teaching on civic and political competence has not been measured beyond self-report surveys. No measures in actual competencies are reported in these studies. This is important because there is evidence that students in active learning classrooms tend to sub-estimate their learning gains, even when they actually learn more (Deslauriers et al., 2019). Second, in both Andersson (2015) and Persson et al. (2020), the difference between the experimental and the control group was not the absence of deliberation but the type of interactions held: while in the experimental groups, students deliberated in small peer-to-peer groups, in the control condition students deliberated first individually and then in whole-class interactions. It can be hypothesized that deliberation was still too present, even between students, in whole-class spaces. Without the control of deliberative moves during lessons, this cannot be ruled out.

To summarize, there is a wide range of empirical evidence suggesting that deliberative argumentation prompts the development of different competencies and skills relevant to sustainable citizenship. The problem is that the empirical evidence remains disperse and fragmented, because even when the research on argumentation skills and knowledge has been conducted within psychology (which is not the case for political competencies, which has mainly been conducted within political and social sciences education), the dialog between these findings is limited. Thus, we lack an integral conception of what deliberative teaching, as a pedagogical practice, can promote, and the joint evaluation of its different benefits is almost non-existent. In each field, we find important knowledge gaps that deserve more research: the effect of different rhetorical styles on skills and knowledge; the effect on social science knowledge, such as human rights, gender, and environmental issues; dosage of interventions and duration of effects; differences according to age, gender, and ethnicity; and learning processes and transfer effects, among others. However, the main challenge is to raise a unified idea of deliberative teaching that clarifies its main characteristics and points clearly to its different benefits, as part of an integral process of teaching and human learning and development. The hope is that such a view could both foster scientific knowledge on the relationship between deliberative teaching and citizenship and impact the political educational agenda more clearly.

\section{DELIBERATIVE TEACHING AS A PEDAGOGICAL EXPERIENCE FOR SUSTAINABLE CITIZENSHIP}

\section{Theoretical Foundations of Deliberative Teaching \\ Deliberation as a Speech Genre}

According to Wiggins (1975), in his Nicomachean Ethics, Aristotle outlines two-related concepts relevant to the notion of deliberation, namely, phronesis (practical wisdom) and boulesis (deliberation). 
Deliberation is understood as a rational process to uncover the best possible means to the desired end (Abizadeh, 2002). It is involved in practical reasoning, which unfolds when a particular and practical problem - for which there is no general and universal answer, and thus it is open to change - requires the best possible decision, from a moral and practical viewpoint (Price, 2011). Although argumentation did not explicitly emerge as being involved in deliberation, from a contemporary point of view it is difficult to conceive of any process of rational evaluation of different alternatives without the use of argumentative language. So, implicitly, argumentation and deliberation have developed as intimately related concepts. Posterior to these classical ideas on deliberation, this term arises again in the context of European Enlightenment, linked to the relationship between free-thinking, democratic values, and public issues, and political decision-making (see Løvlie, 2007).

Deliberation is also a relevant notion in John Dewey's thinking. He positions deliberation not only at the center of an idea of democracy and public life but also as a crucial part of thinking. He developed the idea of deliberation in contrast to a utilitarian notion of deliberation. Following an Aristotelian tradition, deliberation, for Dewey (1922), unfolds when there is a dilemma and an uncertain future. This is typically the case with practical and moral issues, which are open to decisions that have no clear and absolute answers. Deliberation, according to Dewey (1922, p. 139), "has its beginning in troubled activity and its conclusion in (the) choice of a course of action which straightens it out." It is involved in the rational imagination and careful evaluation of different alternative courses of action, based on their consequences. However, we also find deliberation in Dewey's (1910) writings on scientific thinking and education, when a problem may lead to different technical and theoretical solutions, for which, at some point, there is no clear and definitive answer, and for and against need to be imagined and reasoned. Therefore, once a problem or dilemma has been settled, our reading of Dewey $(1910,1916,1922)$ suggests that what defines deliberation is not the practical nature of decisions but: (1) the existence of a problem or dilemma that interrupts and resists habitual ways of thinking; (2) a need to decide on alternative ways of solving the problem or act to restart the flow of thinking; (3) the practice of rational evaluation of the for and against of imagined alternatives and their consequences; and (4) an outcome that is uncertain and indeterminate, so there is no absolute better response beforehand. Moreover, although Dewey does not elaborate upon the notion of argumentation, according to our reading it is inherent to his notion of deliberation (point 3). Finally, it is interesting to note that Dewey (1910) raises a model of thinking based on deliberation; in other words, people deliberate not only interpersonally, to resolve their differences or converge on better solutions, but also with themselves, to deal with personal matters, such as the rational reconstruction of experience.

The idea of deliberation presents in the deliberative teaching approach that has emerged in the field of political science and moral education (Englund, 2016) is informed by Habermas' theory of communicative action and Dewey's views on deliberation and education for democracy. Here, deliberative teaching is intimately linked to the idea of deliberative democracy, referring to how schools and classrooms resemble wider social spaces (Englund, 2016). They, however, unlike Dewey, explicitly recognize the role of argumentation, understood as a procedure of social participation and negotiation. Deliberative teaching in this tradition emerges when contrary views are expressed and discussed through argumentation, with the goal and will of reaching a consensus while attending to differences. Tolerance and acceptance of others' views also characterize this way of teaching, in addition to the possibilities to question traditional views and opportunities for students to communicate with one another with less teacher control. Englund (2016, p. 67) is explicit in viewing deliberative communication as "when conflicts, controversies, confrontations, or different views on any issue arise or are observed and pointed out in the classroom," so he does not restrict deliberation to decisions about courses of action or practical matters.

\section{Deliberation and Argumentation}

Walton (1990), who has had a critical influence on educational scholars, envisages argumentation as a social and verbal activity to resolve (or try to resolve) a conflict of interest or difference of opinion. While arguments are claims that serve the purpose of defending a position against opposition or challenges, argumentation is a goal-directed activity, which requires arguers to intentionally and explicitly use persuasive tools to advance (or demerit) a viewpoint (Walton, 1990) in a given interactional situation or dialog type (Walton, 2006). Deliberation is conceived of as a particular dialog type, characterized by the collective goal of deciding upon the best course of action through the rational examination of possible alternatives.

From a pragma-dialectical point of view (van Eemeren and Grootendorst, 2004), argumentation is the communicative activity of increasing (or decreasing) the acceptability of a given position through the use of justified claims in opposition to other justified claims. In this sense, it is both a reasoning procedure and a communicative action aimed at convincing or presenting the merits of a set of propositions. Like Walton's ideas, the emphasis is on contextual constraints that give argumentation a goal-oriented notion. However, among the van Eemeren's (2013) ideas, the crucial point resides in understanding how argumentative communication types are ratified socially by language usage in prototypical linguistic communities, such as political, organizational, or academic ways of discussion. Although communicative contexts are a combination of different activity types, they can be clustered in different domains of communication by genre, activity type, and concrete speech events. Similarly, deliberation appears as a specific communication genre, a multi-varied cluster of communication, particular in the domain of political communication (van Eemeren and Garssen, 2010; van Eemeren, 2013). Different from other approaches to deliberation, the emphasis relies on the communicative activity type of political agents toward one another (such as presidential debate) in order to convince a popular audience. In van Eemeren's (2013) words, his idea 
differs from that of Walton because it combines deliberation as a discussion procedure with another argumentation genre - the debate - setting the public and political sphere as the scene for deliberation to emerge.

Fairclough's (2017) approach to deliberation and argumentation comes from discourse analysis and political theory. As they point out, there has not yet been a systematic or comprehensive conceptualization of both argumentation and deliberation across different research fields. Their central point (Fairclough, 2017) is that argumentation (along with other language practices) is considered a macro-speech-act type of discourse, while deliberation is a genre within the frame of this macro-level discourse (Fairclough and Mădroane, 2020). While argumentation coexists with narratives, for example, deliberation coexists with negotiation, adjudication, and others. However, argumentation and deliberation are visibly related, as they deal directly with institutionalized decision-making. Taking a discursive approach, they reclaim the role of rhetoric (Fairclough and Fairclough, 2012) in individual and social choice processes between alternative solutions, be it moral, social, or practical problems. For them, rhetoric plays a significant role in how public decision-making is carried out because we cannot dissociate deliberation from its core aspect of persuasion. Most public debates are held by interested agents who have both collective and personal goals; therefore, they can be seen as a rich space for legitimate and illegitimate rhetorical argumentation (Fairclough and Fairclough, 2012). In a sense, this approach approximates both Walton's type of deliberation dialog, oriented toward decision making on alternative options, and van Eemeren's ideas on deliberation as a genre for political debate and audience persuasion.

Although there are differences among these approaches, they converge on conceptualizing deliberation as a particular type of communication activity or speech genre (Bakhtin, 1986), with its own goals, participants, addressees, and compositional styles, in which argumentation, as the activity to formulate arguments and counter-arguments to deal with controversial matters, is used for specific purposes. While argumentation is an abstraction, because it always unfolds through specific genres, deliberation is a family of concrete speech practices. Deliberation, then, involves deliberative argumentation, as a type of argumentation whose goal is to critically and jointly persuade, and be persuaded of, the best possible solution to a given controversial issue. Differences arise when considering the issue at stake, because, regardless of whether or not Walton emphasizes the practical aspects of these issues, Fairclough and Fairclough highlight the institutionalized nature of decision making, while van Eemeren and colleagues ascribe it to political issues.

The ideas of deliberation raised so far overemphasize a rational view of the process. We, however, conceptualize argumentation from a dialogical theory of language (Vološinov, 1929/1986), which acknowledges the affective, positioned and evaluative nature of its unfolding. Moreover, as contradiction is explicitly presented, elaborated, and organized through argumentative language, including deliberative argumentation, it involves identity and motivational processes, and political emotions (Ruitenberg, 2009; Bendixen, 2010), which are raised but also organized, shaped, and transformed through discussions.

Based on these points of view, the presence of deliberation in education is not straightforward. However, as we will argue in the next section, we believe that classroom deliberation is a speech genre that should be conceptualized and taken as a guiding principle to design educative experiences to promote sustainable citizenship.

Therefore, from now on, taking the idea of deliberation raised in the previous section, we understand it as a family of speech genres in which speakers carefully, critically, and affectively consider alternative solutions to controversial and dilemmatic open issues (whether practical or not). They do it through the persuasive and affective imagination, formulation, evaluation, and revision of arguments and counter-arguments, and with the aim of reaching a shared (although plural) view on the matter. Again, it is worth noting that people are involved in argumentative practices from their emotional dispositions, identities, and particular experiences. This may be viewed as a potential threat to deliberation, insofar as speakers may be biased by their individual emotional (Kunda, 1990) and cognitive (Mercier and Sperber, 2017) dispositions and virtues (De Brasi, 2020). However, we consider speakers' affective positionings to be key to engaging and participating in experiences of deliberation that, in turn, are developed by them. Therefore, some authors have emphasized the importance of carefully designing deliberative experiences (Battaly, 2016) to promote individual and collective dispositions.

\section{Deliberative Teaching as a Field of Experience}

We propose to conceive of deliberative teaching as a certain type of educational experience, whether it unfolds in science, language, the arts, maths, social sciences, or civic education, among others. Although deliberative teaching has already been used in the field of political science (also deliberative pedagogy - Shaffer et al., 2017; or deliberative communication - Englund, 2016), with the notion of deliberative democracy as the orienting principle, we propose to borrow the term to articulate a broader and more diverse field of research and professional development in education. As such, deliberative teaching goes beyond a mere intersection of general terms, such as argumentation and education, to summon up initiatives through the common experiences that they offer to students and teachers. The gathering of different initiatives in this field would allow researchers to overcome the fragmentation of our knowledge on the benefits of, and conditions for, this kind of pedagogical experience, enabling a clearer visualization of its potential to promote the development of integral, sustainable, and strong citizens, and thereby - hopefully - achieving a relevant place in educational agendas.

The remarks on Dewey in the previous section are pivotal to our purposes because were deliberation simply a matter of deciding practical problems, some of the revised literature 
would fall out of the field. Many of the studies revised in the previous sections indeed involve students discussing practical matters and imaginary courses of action, whether moral, such as capital punishment (Kuhn et al., 1997) or characters' motivations (Reznitskaya et al., 2009), socio-scientific issues, such as energy use (Felton et al., 2015), or political issues, such as democracy, human rights, or gender (Andersson, 2015). However, there are other problem-based studies involving the discussion of decisions based on the evaluation of arguments and counter-arguments, which are not practical but theoretical (related to concepts and explanations rather than what to do - Asterhan and Schwarz, 2007; Howe, 2009; Larrain et al., 2019; among many others). Our positioning here is that these studies also involved deliberation because, from the students' point of view, they had to decide on dilemmas that were uncertain (they did not have the canonical solutions), and for which they needed to imagine and evaluate critically possible solutions. Moreover, in our view, deliberative teaching has a societal and political value, independent of what is at stake and one's notion of democracy (i.e., deliberative, agonistic, feminist, or other): deliberation is an experience and opportunity to develop key skills, affective positionings, and knowledge into an active citizenship, which, even in violent democracies, may play a key role.

Deliberative teaching as an experience (or family of experiences) is typically characterized by engaging students in activities in which curricular or extracurricular pedagogical goals are attained through intense peer deliberative argumentation around carefully designed controversial problems. It supposes and promotes an inclusive ethos of respectful collaboration and critical engagement. We follow Dewey (1934, p. 42) and his notion of experience as a meaningful unit of a given stream of life, which is felt as a whole, having its own aesthetic quality:

(...) we have an experience when the material experienced runs its course to fulfillment. Then and only then it is integrated within and demarcated in the general stream of experience from other experiences. A piece of work is finished in a way that is satisfactory; a problem receives its solution; a game is played through; and a situation, whether that of eating a meal, playing a game of chess, carrying on a conversation, writing a book, or taking part in a political campaign, is so rounded out that its close is a consummation and not a cessation. Such an experience is a whole and carries with its own individualizing quality and self-sufficiency. It is an experience.

Therefore, we say that deliberative teaching is an experience, in the sense that, from students' subjective viewpoints, it is rounded out with aesthetic quality, with an emotional unity organized by the specific situation in which they participate. It involves clear motives, goals, tasks, and endings; it offers opportunities to feel in a certain way and actively engage with meaningfully presented knowledge; and spaces for social dispute and differentiation, social recognition, and mutual appreciation. Deliberation on any matter or issue involves value judgments and, with them, personal biographies and worldviews: "Deliberation is dramatic and active" (Dewey, 1932, p. 275). Therefore, deliberative teaching involves integral subjects in interdependent subjectivating of meaningfully driven activities, through which skills and knowledge are developed as habits. It is, again, not a rational interplay; rather, it opens the opportunity to enact collective and political emotional positionings (Ruitenberg, 2009) that are key to democratic life.

Deliberative teaching as experience is likely to involve many different and complementary learning and developmental processes discussed in the literature so far: socialization and habituation (McIntosh et al., 2007), appropriation (Anderson et al., 2001), internalization (Larrain et al., 2020), reconstruction of cognitive structures (Howe, 2009), deep elaboration (Eveland and Thomson, 2006), and dramatization and roletaking (Ruitenberg, 2009), among others. In this sense, it is also a learning process that integrates different processes of learning and development, bringing different theoretical and epistemological traditions together.

As such, deliberative teaching calls for whole persons to develop integrally different aspects and dimensions of their personalities and subjectivities in the same stream of life. If we fail to see this as a field, as a family of classroom practices providing students with singular experiences and pointing in the same direction, we are left with bits and pieces but we lose the full picture. In so doing, education loses the crucial potential to develop integral, sustainable citizens. Our argument is that teaching sustainable citizenship requires an integrated view pedagogically implemented - of students' subjective and academic development, which is precisely what deliberative teaching, given all its reported benefits, can offer.

Therefore, our argument is that by inscribing our research in the field of deliberative teaching, on the one hand, we can more clearly recognize dialoguing research on the conditions, characteristics, and effects of this particular type of pedagogical experience, moving the field consistently forward. On the other hand, the idea of deliberative teaching might help to overcome the abstractness of the idea of argumentation, which, from the perspective of teachers and stakeholders, might be counterintuitive, while avoiding the fragmentation and confusion that different available but equivalent labels could reproduce.

\section{Deliberative Teaching, Social Inclusion, and Educational Justice}

Deliberative teaching for sustainable citizenship has a twofold relationship with social inclusion in schools. It assumes that schools are spaces of encountering and sociocultural recognition and appreciation of differences (social, sexual, gender, ethnic, body, and among others), where the idea of normality is disputed and symmetrical participation in educational spaces is seen as key to inclusive education (Slee, 2001). At the same time, it could be seen as an experience to promote social justice.

Social inclusion in education is a complex field. Different traditions have put forward different arguments, emphasizing different identitary and subjective aspects of human development as a focus of inclusion. Therefore, when speaking of social 
inclusion in schools, we are typically pointing out one of many aspects: the inclusion of students with disabilities (Ainscow and Miles, 2008); of socio-economically diverse students (Van Zanten, 2003; Duru-Bellat, 2004; Bonal and Bellei, 2018); the inclusion of gender and sexual diversities (Tinklin et al., 2003), with the concomitant disarticulation of heteronormative cultures within schools (Miller, 2016); and/or the inclusion of ethnically diverse students through the dialogical participation of different national and ethnic group cultures in schools (Dietz, 2012). All of these traditions share a widely acknowledged view of social inclusion as an ethical imperative regarding the role of educational justice in educational systems, offering equal opportunities of participation and recognition and the appreciation of diverse and plural identities of students and their communities, to construct respectful and democratic social relationships and values (Kumashiro, 2001).

The problem is that inclusion is a challenging educational goal, and there is still a gap between these ideal and educational realities (Ainscow et al., 2006). Socioeconomic inclusion is almost impossible in segregated educational systems in which students are separated according to ethnic or socioeconomic conditions (Bonal and Bellei, 2018). There are many studies showing that despite the advances in educational policies to avoid arbitrary gender and sex discrimination in schools, gender gaps, sexism, gender stereotypes and prejudices (Bragg et al., 2018), social exclusion of diverse gender, and sexual identities, still persist (Cumming-Potvin and Martino, 2018). Finally, empirical evidence shows that, although migrant students tend to access education in many countries, they have to face racist and xenophobic practices within schools, in addition to national monocultural curriculums (Sleeter, 2018). In fact, empirical evidence has shown that social or ethnic mixing in schools is not sufficient to deactivate prejudices, stereotypes, and discriminatory practices. On the contrary, different segregation mechanisms may operate (Reay, 2004), such as curricular tracking (Sevilla and Polesel, 2020), groupings by friendship or family influence in school schedules (Reay and Ball, 1998; Van Zanten, 2003).

Fragmentation of the field of social inclusion in education does not help it to advance integrally in educational justice. The different traditions mentioned claim different identities, expressing particular sociopolitical debates. This has had consequences for educational policies, insofar as they have tended to regulate specific aspects of subjective development separately: disability, socioeconomic disadvantage, gender, or ethnic diversity. These policies penetrate schools in a disperse and disarticulate way, reinforcing the stereotypes of teachers and principals, with less impact on schools' capacity to promote democratic relations in diverse settings (Slee, 2001). Therefore, pedagogical practices offering integral experiences of inclusion in schools are both scarce and compulsory (Frankenberg and Orfield, 2012; Blokland and Nast, 2014).

Social inclusion is not an end in itself (Slee, 2001) but a baseline scenario for basic conditions for educational justice. Following Dewey's (1916) legacy on democratic education, education involves a process of cultural reconstruction in which all, without exclusion, should find recognition of their different individual experiences and plural identities and their contribution to collective life. The ability to raise common goals, collaboratively and symmetrically, in the absence of dominant hegemonies is what characterizes real inclusive school cultures (Dewey, 1916; Slee, 2001). However, this recognition needs to be institutionally facilitated; it cannot simply be demanded as an ethical mandate relying on students' individual socio-emotional skills. Beyond putting students together, and following Juvonen et al. (2019), Nishina et al. (2019), and the available empirical evidence (Aronson and Bridgeman, 1979; Sharan, 1980; Graham, 2018; García-Carrión et al., 2020), we argue that schools need to provide students with opportunities to have engaging and meaningful educational experiences of encountering and collaborative thoughtful activity with their peers. This allows them to reconstruct a common frame for identitary articulation (Rojas et al., 2016), thereby promoting friendship (Graham, 2018; Juvonen et al., 2019) and building integrally inclusive schools.

We argue that deliberative teaching, although requiring diversity as a basic condition, can also be conceived of as an inclusive pedagogical experience, that is, enhancing and deepening social inclusion in schools. Deliberative teaching offers possibilities of real encounters and mutual knowledge and recognition, in which real differences are expressed and articulated. On the other hand, it offers spaces to focus on the ideas and arguments, juxtaposing in a meaningful way the worldviews and subjectivities of diverse others, offering the chance to represent others' perspectives and appreciate their contribution, and in the process breaking down prejudices. This is one way in which peer effects (Van Ewijk and Sleegers, 2010) can act to diminish academic segregation under socially heterogeneous conditions, promoting positive outcomes to both socioculturally disadvantaged (Van Zanten, 2003; Duru-Bellat, 2004; Bonal and Bellei, 2018) and advantaged students (Orfield, 2001; Orfield and Frankenberg, 2013). However, these practices and experiences require a broader view on education for democracy, which can involve an idea of deliberative education. In such a view, coherent with Dewey's (1916) ideas, deliberation should be seen as a practice beyond classrooms, involving practices of teachers' professional development, curricular and pedagogical decision making, and practices of articulation of all school actors. More importantly, deliberative teaching practices need to be developed in a broader framework of democratic and educational justice, where everybody's dignity and experience are valued and used to raise collective norms and values (Feu et al., 2017; Belavi and Murillo, 2020).

\section{DISCUSSION}

The main argument of this paper is that deliberative teaching, as a field grouping diverse research on classroom experiences involving peer deliberative argumentation, can visualize the affordances of these transversal pedagogical practices to promote the development of integral subjects into sustainable citizens. We observe that the different traditions we have explored through the paper are sending a clear but insufficiently 
heard message: Classroom deliberative argumentation can have crucial benefits for citizenship, whether the object of deliberation is scientific, mathematical, social, artistic, or moral. These benefits become really meaningful for citizenship development only when one overcomes the fragmentation of the different dimensions studied as benefits (argumentation skills, knowledge, politics, and civic competence) and of the different political agendas behind the study of social inclusion in education. The configuration of deliberative teaching as a field can be the first step into a necessary integral view of citizens' development.

The idea of deliberative teaching is not new. The label has already been used in political science and social science education (Andersson, 2015) and has its roots in Dewey's thinking. Moreover, research on classroom dialog and deliberative democracy has already been proposed [Erduran and Kaya, 2016; Michaels et al. (2008)]. Our proposal, however, is to use this notion to give to an implicit and disarticulated field a common identity that enhances the possibilities of diverse research traditions and contributions in order to achieve mutual recognition, convergence, and educational impact. Moreover, although we know about the benefits of deliberative teaching, there are still relevant knowledge gaps within each sub-field. For instance, experimental evidence on skills has been found mainly in countries in the global North, and questions about dosage - how long and intense interventions should be - and transfer remain (see Reznitskaya et al., 2012). Evidence on knowledge of social issues, such as human rights and gender have been less well attended, and learning mechanisms are still insufficiently understood (Larrain et al., 2019). The relationship between deliberative argumentation and epistemological knowledge is also a persistent challenge. The effect of deliberation on political competence beyond self-reports, and the differential role of peer interaction (Persson et al., 2020), still need to be appropriately studied. In addition, although the relation between deliberative teaching and social justice makes perfect sense, it has not been extensively empirically studied, mainly because social inclusion research agendas have not yet crossed over to the other research traditions presented here. More importantly, the assumption that deliberative teaching can prompt many benefits at the same time (Iordanou et al., 2019; Larrain et al., 2020), and that these benefits are relevant to sustainable citizenship, still needs to be empirically tested. However, this requires an integrated view on the matter.

It is worth noting that promoting knowledge plays a key role in the deliberative teaching potential to develop sustainable citizens. This is the case not only because of what has already been discussed, for instance, the role of knowledge and scientific literacy in public evidence-based decision making. In addition, we argue that climate action, social inclusion, and justice also require knowledge construction on key issues. For instance, regarding sexual and gender inclusion, if students are told from a moral point of view that they should respect others and avoid discrimination of women or other gender identities, they can accept or reject it, because it has been relayed as a dogma. Instead, if that general idea is transformed into a situated problem and is open to deliberation, with the expression and argumentation of many points of view - even ones that seem unacceptable - this can contribute to a deep understanding of the tensions involved, legitimating normative and legal decisions. This of course imposes tensions on teachers, who may perceive it as difficult to orchestrate and consolidate discussions in which different points of view, including unacceptable ones, are expressed and promoted. Although there have been several initiatives to promote and study professional development for scientific argumentation in classrooms (Osborne et al., 2019), more studies are needed.

Deliberative teaching as pedagogical experiences could be accused of idealism and liberalism, and for good reason. Authors have warned against deliberative pedagogies as disciplining practices for a liberal view of citizens (DesRoches and Ruitenberg, 2018). Michaels et al. (2008) highlight the real experiences of deliberation in classrooms in which social and cultural capital (see Dubet, 2004; Bourdieu, 2011) could differentially shape students' participation and, in turn, reproduce pre-existing inequalities. Moreover, status and power asymmetries operating in peers' social relations render deliberation experiences dependent on social structures. This has been illustrated by middle-class students doing better in socially mixed public schools, enlarging the initial differences (Jansson et al., 2020; Mendoza, 2020). We need to pay special attention to prevent deliberative teaching practices from promoting more segregation by institutionalizing and privileging one type of social participation over the diversity of political agency.

However, we think that there are ways to make deliberation central to the educational experience without simply reproducing a certain type of citizen or social inequalities. In other words, deliberative teaching is not necessarily liberal, rational, and elitist. If deliberative discussions are not only cosmetic but also involve as objects of a dispute the social conditions of life and education, we believe that they are still one of the best ways to break students' asymmetric power positions and agencies. A critical school curriculum, flexible and permeable to diverse life experiences, and a culture of critical and collaborative school governance, prioritizing the redistribution of students' learning opportunities (Belavi and Murillo, 2020), have been considered key to deliberative professional cultures that increase the probabilities of social inclusion at school (Mabovula, 2009). Therefore, again, deliberative teaching needs to be part of a general framework of education for social justice and democracy at the school level, in which social differences are visible and gaps are acknowledged and not simply accepted.

Deliberative teaching might appear to be unreachable within the current educational systems, organized by high-stakes accountability policies and testing agendas, which in some countries co-exist with mercantile policies. These educational systems have increased their social segregation (Murillo and Martinez-Garrido, 2020), threatening social inclusion goals (Frankenberg and Orfield, 2012; Bonal and Bellei, 2018; Murillo and Martinez-Garrido, 2020), and reducing innovative teaching practices (Mathison and Freeman, 2003; Parcerisa and Falabella, 2017) and curriculum richness (Au, 2007). Deliberative teaching 
should consider these sociopolitical conditions, identifying how they tension its unfolding (Katsh-Singer et al., 2016; Ydesen et al., 2020) and developing an understanding of how to design situated deliberative teaching practices that consider teachers' labor conditions (Ball and Olmedo, 2013). Otherwise, it could contribute to concerns among teachers and principals about how to articulate different educational goals (Ryan, 2006, 2010). Furthermore, to imagine deliberative teaching, extracurricular online instances whereby students can interact with students from different schools and backgrounds could be a way to promote social justice within segregated educational systems.

Deliberative teaching, as we understand it, does not imply a notion of deliberative democracy, in which conflicts are rationally solved and the best possible options are achieved. We value and acknowledge conflict and tension as part of any democratic life, with no need to dissolve them. However, we are not arguing for a fully agonistic view of democracy (Mouffe, 2014). As South American scholars, we are committed to the need to recognize conflicts as part of social life, but acknowledging the challenge and the need to handle them in a way that enables a common and sustainable life. Thus, developing skills and political affections to achieve this is key. In that sense, our view of sustainable citizenship departs from a notion of adaptive or individualized citizenship, establishing intimate relations with Veugelers' (2020) idea of critical-democratic citizenship, or Rapanta et al's (2020) notion of culturally literate citizens, both of which recognize argumentative deliberation as a relevant means of education.

The argument that is central to this paper is the need to concur with a field based on the notion of deliberative teaching. Reasonable counter-arguments could be put forward to challenge the proposal, questioning the real effect of a new label, considering that there are ones already available that have failed to solve the fragmentation of current research (for

\section{REFERENCES}

Abizadeh, A. (2002). The passions of the wise: "Phronêsis", rhetoric, and Aristotle's passionate practical deliberation. Rev. Metaphys. 56, 267-296.

Ainscow, M., Booth, T., and Dyson, A. (2006). Inclusion and the standards agenda: negotiating policy pressures in England. Int. J. Incl. Educ. 10, 295-308. doi: 10.1080/13603110500430633

Ainscow, M., and Miles, S. (2008). Making education for all inclusive: where next?. Prospects 38, 15-34. doi: 10.1007/s11125-008-9055-0

Alvis, L. M., and Metzger, A. (2020). Dimensions of adolescent civic reasoning: an examination of individual differences. J. Adolesc. Res. 35, 309-340. doi: $10.1177 / 0743558419862485$

Anderson, R. C., Nguyen-Jahiel, K., McNurlen, B., Archodidou, A., Kim, S. Y., Reznitskaya, A., et al. (2001). The snowball phenomenon: spread of ways of talking and ways of thinking across groups of children. Cogn. Instr. 19, 1-46. doi: 10.1207/S1532690XCI1901_1

Andersson, K. (2015). Deliberative teaching: effects on students' democratic virtues. Scand. J. Educ. Res. 59, 604-622. doi: 10.1080/00313831.2014.965789

Andriessen, J. E. B., and Schwarz, B. B. (2009). "Argumentative design" in Argumentation and Education. eds. N. M. Mirza and A. N. Perret-Clermont (New York: Springer), 145-174.

Aronson, E., and Bridgeman, D. (1979). Jigsaw groups and the desegregated classroom: in pursuit of common goals. Personal. Soc. Psychol. Bull. 5, 438-446. doi: $10.1177 / 014616727900500405$ instance, dialogical teaching or inclusive education). Our answer would be that these fields include broader conversations, which are not focused on deliberative argumentation. As we argue that deliberative argumentation has several benefits to citizenship education, this is what we are proposing to bring to the fore as a common ground. So, it is not a question of simply labeling or establishing a new small feudo for a given research agenda. It is about inviting more people to take part in the conversation that is already happening but clouded by many other conversations going on in these fields. The invitation is to recognize a non-exclusive and superposing field of interlocution that can provide us with specific affordances to discuss issues of deliberative teaching and its impact on citizenship education.

\section{AUTHOR CONTRIBUTIONS}

AL, GF, and MR drafted the first version of the manuscript and reviewed the final one. All authors contributed to the article and approved the submitted version.

\section{FUNDING}

This paper was supported financially by the Fondo Nacional de Desarrollo Científico y Tecnológico (FONDECYT) and Agencia Nacional de Investigación y Desarrollo de Chile (ANID), grants 1210264,3190488 , and 1180886 .

\section{ACKNOWLEDGMENTS}

The authors wish to thank Alina Reznitskaya for her careful reading and comments and Amanda George for her proofreading.

Asterhan, C. S., and Babichenko, M. (2015). The social dimension of learning through argumentation: effects of human presence and discourse style. J. Educ. Psychol. 107, 740-755. doi: 10.1037/edu0000014

Asterhan, C. S., and Hever, R. (2015). Learning from reading argumentative group discussions in Facebook: rhetoric style matters (again). Comput. Hum. Behav. 53, 570-576. doi: 10.1016/j.chb.2015.05.020

Asterhan, C. S., and Schwarz, B. B. (2007). The effects of monological and dialogical argumentation on concept learning in evolutionary theory. J. Educ. Psychol. 99, 626-639. doi: 10.1037/0022-0663.99.3.626

Asterhan, C. S., and Schwarz, B. B. (2016). Argumentation for learning: welltrodden paths and unexplored territories. Educ. Psychol. 51, 164-187. doi: 10.1080/00461520.2016.1155458

$\mathrm{Au}$, W. (2007). High-stakes testing and curricular control: a qualitative metasynthesis. Educ. Res. 36, 258-267. doi: 10.3102/0013189X07306523

Aydeniz, M. A., and Dogan, A. (2016). Exploring the impact of argumentation on pre-service science teachers' conceptual understanding of chemical equilibrium. Chem. Edu. Res. Pract. 7, 111-119. doi: 10.1039/ c5rp00170f

Bakhtin, M. M. (1986). “The problem of speech genres" in Speech Genres and Other Late Essays. Vol. 17 (Austin: University of Texas Press), 60-102.

Ball, S. J., and Olmedo, A. (2013). Care of the self, resistance and subjectivity under neoliberal governmentalities. Crit. Stud. Edu. 54, 85-96. doi: 10.1080/ 17508487.2013.740678 
Battaly, H. (2016). Developing virtue and rehabilitating vice: worries about self-cultivation and self-reform. J. Moral Educ. 45, 207-222. doi: 10.1080/03057240.2016.1195732

Beaumont, E., Colby, A., Ehrlich, T., and Torney-Purta, J. (2006). Promoting political competence and engagement in college students: an empirical study. J. Pol. Sci. Edu. 2, 249-270. doi: 10.1080/15512160600840467

Belavi, G., and Murillo, J. (2020). Democracia y Justicia Social en las Escuelas: dimensiones para Pensar y Mejorar la Práctica Educativa. Revista Iberoamericana sobre Calidad, Eficacia y Cambio en Educación 18, 5-28. doi: 10.15366/ reice2020.18.3.001

Bendixen, L. D. (2010). Argumentation, anger, and action: citizenship education in and out of the classroom. J. Peace Edu. Social Justice 4, 108-119.

Bennion, E. A., and Laughlin, X. E. (2018). Best practices in civic education: lessons from the Journal of Political Science Education. J. Pol. Sci. Edu. 14, 287-330. doi: 10.1080/15512169.2017.1399798

Blokland, J., and Nast, T. (2014). From public familiarity to comfort zone: the relevance of absent ties for belonging in Berlin's mixed neighbourhoods. Int. J. Urban Reg. Res. 38, 1142-1159. doi: 10.1111/1468-2427.12126

Bonal, X., and Bellei, C. (2018). Understanding School Segregation: Patterns, Causes and Consequences of Spatial Inequalities in Education. London: Bloomsbury Academic.

Bourdieu, P. (2011). Las estrategias de la reproducción social [Strategies of Social Reproduction]. México: Siglo XXI editores.

Bouyias, Y., and Demetriadis, S. (2012). Peer-monitoring vs. micro-script fading for enhancing knowledge acquisition when learning in computer-supported argumentation environments. Comput. Educ. 59, 236-249. doi: 10.1016/j. compedu.2012.01.001

Bragg, S., Renold, E., Ringrose, J., and Jackson, C. (2018). "More than boy, girl, male, female": exploring young people's views on gender diversity within and beyond school contexts. Sex Educ. 18, 420-434. doi: 10.1080/ 14681811.2018.1439373

Crowell, A., and Kuhn, D. (2014). Developing dialogic argumentation skills: a 3-year intervention study. J. Cogn. Dev. 15, 363-381. doi: 10.1080/ 15248372.2012.725187

Cumming-Potvin, W., and Martino, W. (2018). Countering heteronormativity and cisnormativity in Australian schools: examining English teachers' reflections on gender and sexual diversity in the classroom. Teach. Teach. Educ. 74, 35-48. doi: $10.1016 /$ j.tate.2018.04.008

De Brasi, L. (2020). Argumentative deliberation and the development of intellectual humility and autonomy in the classroom. Cogency 12, 13-37. doi: 10.32995/ cogency.v12i1.339

Deslauriers, L., McCarty, L. S., Miller, K., Callaghan, K., and Kestin, G. (2019). Measuring actual learning versus feeling of learning in response to being actively engaged in the classroom. Proc. Natl. Acad. Sci. 116, 19251-19257. doi: $10.1073 /$ pnas. 1821936116

DesRoches, S. J., and Ruitenberg, C. W. (2018). "Chantal Mouffe," in International Handbooks of Education (Springer), 283-294.

Dewey, J. (1910). How We Think. Boston: D. C. Heath \& Co. Publishers.

Dewey, J. (1916). "Democracy and education," in The Middle Works of John Dewey, 1899-1924 (Carbondale, IL: Southern Illinois University Press).

Dewey, J. (1922). "Human nature and conduct," in The Middle Works of John Dewey, 1899-1924 (Carbondale, IL: Southern Illinois University Press).

Dewey, J. (1932). "Ethics," in The Later Works of John Dewey, 1925-1953 (Carbondale, IL: Southern Illinois University Press).

Dewey, J. (1934). "Art as experience," in The Later Works of John Dewey, 1925-1953 (Carbondale, IL: Southern Illinois University Press).

Dewey, J. (1944/1989). "The democratic faith and education," in The Later Works of John Dewey, 1925-1953 (Carbondale, IL: Southern Illinois University Press).

Dietz, D. (2012). Multiculturalismo, interculturalidad y diversidad en educación. Una aproximación antropológica [Multiculturalism, Interculturality and Diversity in Education. An Anthropological Approach]. México: Fondo de Cultura Económica.

Dubet, F. (2004). L’école des chances, quest-ce que c’est une école juste. France: édition du Seuil et La République des Idées.

Duru-Bellat, M. (2004). La ségrégation sociale à lécole: faits et effets. Diversité Ville École Intégration 139, 73-80.

Englund, T. (2016). On moral education through deliberative communication. J. Curric. Stud. 48, 58-76. doi: 10.1080/00220272.2015.1051119
Erduran, S., and Kaya, E. (2016). Scientific argumentation and deliberative democracy: an incompatible mix in school science? Theory Pract. 55, 302-310. doi: 10.1080/00405841.2016.1208067

Eveland, W. P. Jr. (2004). The effect of political discussion in producing informed citizens: the roles of information, motivation, and elaboration. Polit. Commun. 21, 177-193. doi: $10.1080 / 10584600490443877$

Eveland, W. P. Jr., and Thomson, T. (2006). Is it talking, thinking, or both? A lagged dependent variable model of discussion effects on political knowledge. J. Commun. 56, 523-542. doi: 10.1111/j.1460-2466.2006.00299.x

Fairclough, I. (2017). "Deliberative discourse," in The Routledge Handbook of Critical Discourse Studies (London: Routledge), 242-256.

Fairclough, I., and Fairclough, N. (2012). Political Discourse Analysis: A Method for Advanced Students. London: Routledge.

Fairclough, I., and Mădroane, I. D. (2020). An argumentative approach to "framing" framing, deliberation and action in an environmental conflict. Co-herencia 17, 119-158. doi: 10.17230/co-herencia.17.32.5

Felton, M., García-Mila, M., and Gilabert, S. (2009). Deliberation versus dispute: the impact of argumentative discourse goals on learning and reasoning in the science classroom. Informal Logic 29, 417-446. doi: 10.22329/il.v29i4.2907

Felton, M., Garcia-Mila, M., Villarroel, C., and Gilabert, S. (2015). Arguing collaboratively: argumentative discourse types and their potential for knowledge building. Br. J. Educ. Psychol. 85, 372-386. doi: 10.1111/bjep.12078

Felton, M., and Kuhn, D. (2001). The development of argumentative discourse skill. Discourse Process. 32, 135-153. doi: 10.1207/S15326950DP3202\&3_03

Feu, J., Serra, C., Canimas, J., et al. (2017). Democracy and education: a theoretical proposal for the analysis of democratic practices in schools. Stud. Philos. Educ. 36, 647-661. doi: 10.1007/s11217-017-9570-7

Frankenberg, E., and Orfield, G. (eds.) (2012). The Resegregation of Suburban Schools: A Hidden Crisis in American Education. Cambridge, MA: Harvard Education Press.

García-Carrión, R., López de Aguileta, G., Padrós, M., and Ramis-Salas, M. (2020). Implications for social impact of dialogic teaching and learning. Front. Psychol. 11:140. doi: 10.3389/fpsyg.2020.00140

Graham, S. (2018). Race/ethnicity and social adjustment of adolescents: how (not if) school diversity matters. Educ. Psychol. 53, 64-77. doi: $10.1080 / 00461520.2018 .1428805$

Hess, D. E., and McAvoy, P. (2014). The Political Classroom: Evidence and Ethics in Democratic Education. London: Routledge.

Howe, C. (2009). Collaborative group work in middle childhood: joint construction, unresolved contradiction and the growth of knowledge. Hum. Dev. 39, 71-94. doi: 10.1159/000215072

Howe, C., Tolmie, A., Duchak-Tanner, V., and Rattay, C. (2000). Hypothesistesting in science: group consensus and the acquisition of conceptual and procedural knowledge. Learn. Instr. 10, 361-391. doi: 10.1016/ S0959-4752(00)00004-9

Howe, C., and Zachariou, A. (2019). Small-group collaboration and individual knowledge acquisition: the processes of growth during adolescence and early adulthood. Learn. Instr. 60, 263-274. doi: 10.1016/j.learninstruc.2017. 10.007

Iordanou, K., and Kuhn, D. (2020). Contemplating the opposition: does a personal touch matter? Discourse Process. 57, 343-359. doi: 10.1080/0163853X.2019.1701918

Iordanou, K., Kuhn, D., Matos, F., Shi, Y., and Hemberger, L. (2019). Learning by arguing. Learn. Instr. 63, 101-207. doi: 10.1016/j.learninstruc.2019.05.004

Jansson, F., Birkelund, G. E., and Lillehagen, M. (2020). Segregation within school classes: detecting social clustering in choice data. PLoS One 15:e0233677. doi: 10.1371/journal.pone.0233677

Jermann, P., and Dillenbourg, P. (2003). Elaborating New Arguments Through a CSCL Script in Arguing to Learn. Netherlands: Springer, 205-226.

Juvonen, J., Lessard, R., Rastogi, R., Schacter, H., and Smith, D. (2019). Promoting social inclusion in educational settings: challenges and opportunities. Educ. Psychol. 54, 250-270. doi: 10.1080/00461520.2019.1655645

Katsh-Singer, R., McNeill, K. L., and Loper, S. (2016). Scientific argumentation for all? Comparing teacher beliefs about argumentation in high, mid, and low socioeconomic status schools. Sci. Educ. 100, 410-436. doi: 10.1002/ sce. 21214

Kaya, E. (2013). Argumentation practices in classroom: pre-service teachers' conceptual understanding of chemical equilibrium. Int. J. Sci. Educ. 35, 1139-1158. doi: 10.1080/09500693.2013.770935 
Kuhn, D., Cheney, R., and Weinstock, M. (2000). The development of epistemological understanding. Cogn. Dev. 15, 309-328. doi: 10.1016/ S0885-2014(00)00030-7

Kuhn, D., and Crowell, A. (2011). Dialogic argumentation as a vehicle for developing young adolescents' thinking. Psychol. Sci. 22, 545-552. doi: $10.1177 / 0956797611402512$

Kuhn, D., Shaw, V., and Felton, M. (1997). Effects of dyadic interaction on argumentative reasoning. Cogn. Instr. 15, 287-315. doi: 10.1207/ s1532690xci1503_1

Kuhn, D., and Udell, W. (2003). The development of argument skills. Child Dev. 74, 1245-1260. doi: 10.1111/1467-8624.00605

Kumashiro, K. (2001). "Posts"' perspectives on anti-oppressive education in social studies, English, mathematics, and science classrooms. Educ. Res. 30, 3-12. doi: 10.3102/0013189X030003003

Kunda, Z. (1990). The case for motivated reasoning. Psychol. Bull. 108, 480-498. doi: $10.1037 / 0033-2909.108 .3 .480$

Kurian, P. A., Munshi, D., and Bartlett, R. V. (2014). Sustainable citizenship for a technological world: negotiating deliberative dialectics. Citizsh. Stud. 18, 435-451. doi: 10.1080/13621025.2014.905284

Kyle, W. C. (2020). Expanding our views of science education to address sustainable development, empowerment, and social transformation. Discip. Interdiscipl. Sci. Edu. Res. 2, 1-9. doi: 10.1186/s43031-019-0018-5

Larrain, A., Freire, P., Grau, V., López, P., Salvat, I., Silva, M., et al. (2018). The effect of peer-group argumentative dialogue on delayed gains in scientific content knowledge. New Direct. Child Adoles. Develop. 162, 67-87. doi: $10.1002 / \mathrm{cad} .20263$

Larrain, A., Freire, P., López, P., and Grau, V. (2019). Counter-arguing during curriculum-supported peer interaction facilitates middle-school students' science content knowledge. Cognit. Instruction 37, 453-482. doi: 10.1080/07370008.2019.1627360

Larrain, A., Singer, V., Strasser, K., Howe, C., López, P., Pinochet, J., et al. (2020). Argumentation skills mediate the effect of peer argumentation on content knowledge in middle-school students. J. Educ. Psychol. doi: 10.1037/ edu0000619

Latimer, C., and Hempson, K. M. (2012). Using deliberation in the classroom: a teaching pedagogy to enhance student knowledge, opinion formation, and civic engagement. J. Pol. Sci. Edu. 8, 372-388. doi: 10.1080/15512169. 2012.729447

Lin, H. S., Hong, Z. R., and Lawrenz, F. (2012). Promoting and scaffolding argumentation through reflective asynchronous discussions. Comput. Educ. 59, 378-384. doi: 10.1016/j.compedu.2012.01.019

Løvlie, L. (2007). "Education for deliberative democracy" in Education in the Era of Globalization (Dordrecht: Springer), 123-145.

Mabovula, N. (2009). Giving voice to the voiceless through deliberative democratic school governadnce. S. Afr. J. Educ. 29, 219-233. doi: 10.15700/saje. v29n2a162

Mathison, S., and Freeman, M. (2003). Constraining elementary teachers' work: dilemmas and paradoxes created by state mandated testing. Edu. Policy Anal. 11:34. doi: 10.14507/epaa.v11n34.2003

McIntosh, H., Hart, D., and Youniss, J. (2007). The influence of family political discussion on youth civic development: which parent qualities matter? PS: Polit. Sci. Polit. 40, 495-499. doi: 10.1017/S1049096507070758

Mendoza, M. J. (2020). Parents' and teachers' dispositions towards social class difference and mix. A qualitative case study in two socioeconomically diverse Chilean schools. doctoral thesis. London: University College London.

Mercer, N. (2009). "Developing argumentation: lessons learned in the primary school" in Argumentation and Education. eds. N. Muller Mirza and A. N. Perret-Clermont (Boston, MA: Springer), 177-194.

Mercer, N., and Littleton, K. (2007). Dialogue and the Development of Children's Thinking. London: Routledge.

Mercier, H., and Sperber, D. (2017). The Enigma of Reason. Cambridge, MA: Harvard University Press.

Merkel, W. (2014). Is there a crisis of democracy? Democratic Theory 1, 11-25. doi: $10.3167 /$ dt.2014.010202

Michaels, S., O'Connor, C., and Resnick, L. B. (2008). Deliberative discourse idealized and realized: accountable talk in the classroom and in civic life. Stud. Philos. Educ. 27, 283-297. doi: 10.1007/s11217-007-9071-1

Miller, S. J. (2016). Teaching, Affirming and Recognizing Trans* and Gender Creative Youth. US: Palgrave Macmillan.
Mouffe, C. (2014). "Democratic politics and conflict: an agonistic approach" in Political Power Reconsidered: State Power and Civic Activism Between Legitimacy and Violence. ed. M. Lakitsch (Wien, Austria: LIT-Verlag), 17-29.

Murillo, J., and Martinez-Garrido, C. (2020). Segregación Escolar como Opresión. REICE 18, 5-8. doi: 10.15366/reice2020.18.4

Nishina, A., Lewis, J. A., Bellmore, A., and Witkow, M. R. (2019). Ethnic diversity and inclusive school environments. Educ. Psychol. 54, 306-321. doi: $10.1080 / 00461520.2019 .1633923$

Noroozi, O., Biemans, H., and Mulder, M. (2016). Relations between scripted online peer feedback processes and quality of written argumentative essay. Internet High. Educ. 31, 20-31. doi: 10.1016/j.iheduc.2016.05.002

Noroozi, O., Weinberger, A., Biemans, H. J., Mulder, M., and Chizari, M. (2013). Facilitating argumentative knowledge construction through a transactive discussion script in CSCL. Comput. Edu. 61, 59-76. doi: 10.1016/j. compedu.2012.08.013

Nussbaum, E. M., Hartley, K., Sinatra, G. M., Reynolds, R. E., and Bendixen, L. D. (2004). Personality interactions and scaffolding in on-line discussions. J. Educ. Comput. Res. 30, 113-137. doi: 10.2190/H8P4-QJUF-JXME-6JD8

Nussbaum, E. M., Winsor, D. L., Aqui, Y. M., and Poliquin, A. M. (2007). Putting the pieces together: online argumentation vee diagrams enhance thinking during discussions. Int. J. Comput.-Support. Collab. Learn. 2, 479-500. doi: 10.1007/s11412-007-9025-1

Orfield, G. (2001). Schools More Separate: Consequences of a Decade of Resegregation. Cambridge, MA: Harvard University.

Orfield, G., and Frankenberg, E. (2013). Educational Delusions? Berkeley: University of California Press.

Osborne, J. F., Borko, H., Fishman, E., Gomez Zaccarelli, F., Berson, E., Busch, K. C., et al. (2019). Impacts of a practice-based professional development program on elementary teachers' facilitation of and student engagement with scientific argumentation. Am. Educ. Res. J. 56, 1067-1112. doi: $10.3102 / 0002831218812059$

Parcerisa, L., and Falabella, A. (2017). La consolidación del estado evaluador a través de políticas de rendición de cuentas: Trayectorias, producción y tensiones en el sistema educativo. Archivos Analíticos de Políticas Educativas 25, 1-4. doi: 10.14507/epaa.25.3177

Persson, M., Andersson, K., Zetterberg, P., Ekman, J., and Lundin, S. (2020). Does deliberative education increase civic competence? Results from a field experiment. J. Exp. Polit. Sci. 7, 199-208. doi: 10.1017/XPS.2019.29

Price, A. W. (2011). "Aristotle on the ends of deliberation," in Moral Psychology and Human Action in Aristotle. eds. M. Pakaluk and G. Pearson (Oxford: Oxford University Press), 135-158.

Rapanta, C., Vrikki, M., and Evagorou, M. (2020). Preparing culturally literate citizens through dialogue and argumentation: rethinking citizenship education. Curriculum J. doi: 10.1002/curj.95

Reay, D. (2004). Education and cultural capital: the implications of changing trends in education policies. Cult. Trends 13, 73-86. doi: 10.1080/ 0954896042000267161

Reay, D., and Ball, S. (1998). Making their minds up: family dynamics of school choice. Br. Educ. Res. J. 24, 431-448. doi: 10.1080/0141192980240405

Reznitskaya, A., Glina, M., Carolan, B., Michaud, O., Rogers, J., and Sequeira, L. (2012). Examining transfer effects from dialogic discussions to new tasks and contexts. Contemp. Educ. Psychol. 37, 288-306. doi: 10.1016/j. cedpsych.2012.02.003

Reznitskaya, A., Kuo, L., Clark, A., Miller, B., Jadallah, M., Anderson, R. C., et al. (2009). Collaborative reasoning: a dialogic approach to group discussion. Camb. J. Educ. 39, 29-48. doi: 10.1080/03057640802701952

Rojas, M. T., Falabella, A., and Alarcón, P. (2016). Inclusión social en las escuelas: estudio de prácticas pedagógicas inclusivas y proyecciones para enfrentar un escenario sin copago y selección escolar. Working paper 1-119. Available at: https://www.supereduc.cl/wp-content/uploads/2017/01/Inclusionsocial-en-las-escuelas.pdf

Ruitenberg, C. W. (2009). Educating political adversaries: Chantal Mouffe and radical democratic citizenship education. Stud. Philos. Educ. 28, 269-281. doi: $10.1007 / \mathrm{s} 11217-008-9122-2$

Ryan, J. (2006). Inclusive leadership and social justice for schools. Leadersh. Policy Sch. 5, 3-17. doi: 10.1080/15700760500483995

Ryan, J. (2010). Promoting social justice in schools: principals' political strategies. Int. J. Leadersh. Educ. 13, 357-376. doi: 10.1080/13603124.2010.503281 
Ryfe, D. M. (2005). Does deliberative democracy work? Annu. Rev. Polit. Sci. 8, 49-71. doi: 10.1146/annurev.polisci.8.032904.154633

Sandoval, W. A., Greene, J. A., and Bråten, I. (2016). Understanding and promoting thinking about knowledge: origins, issues, and future directions of research on epistemic cognition. Rev. Res. Educ. 40, 457-496. doi: $10.3102 / 0091732 \times 16669319$

Sevilla, P., and Polesel, J. (2020). Vocational education and social inequalities in within- and between-school curriculum tracking. Compare: J. Comp. Int. Edu. 1-19. [Preprint]. doi: 10.1080/03057925.2020.1798214

Shaffer, T. J., Longo, N. V., Manosevitch, I., and Thomas, M. S. (eds.) (2017). Deliberative Pedagogy: Teaching and Learning for Democratic engagement. Lansing, MI: MSU Press.

Sharan, S. (1980). Cooperative learning in small groups: recent methods and effects on achievement, attitudes, and ethnic relations. Rev. Educ. Res. 50, 241-271. doi: 10.3102/00346543050002241

Slee, R. (2001). Driven to the margins: disabled students, inclusive schooling and the politics of possibility. Camb. J. Educ. 31, 385-397. doi: 10.1080/ 03057640120086620

Sleeter, C. (2018). Multicultural education past, present, and future: struggles for dialog and power-sharing. Int. J. Multicult. Edu. 20, 5-20. doi: 10.18251/ ijme.v20i1.1663

Stegmann, K., Weinberger, A., and Fischer, F. (2007). Facilitating argumentative knowledge construction with computer-supported collaboration scripts. Int. J. Comput.-Support. Collab. Learn. 2, 421-447. doi: 10.1007/s11412-007-9028-y

Tinklin, T., Croxford, L., Ducklin, A., and Frame, B. (2003). Inclusion: a gender perspective. Policy Futures Edu. 1, 640-652. doi: 10.2304/pfie.2003.1.4.3

Tolmie, A., Howe, C., Mackenzie, M., and Greer, K. (1993). Task design as an influence on dialogue and learning: primary school group work with object flotation. Soc. Dev. 2, 183-201. doi: 10.1111/j.1467-9507.1993.tb00013.x

Tsovaltzi, D., Judele, R., Puhl, T., and Weinberger, A. (2017). Leveraging social networking sites for knowledge co-construction: positive effects of argumentation structure, but premature knowledge consolidation after individual preparation. Learn. Instr. 52, 161-179. doi: 10.1016/j. learninstruc.2017.06.004

van Eemeren, F., and Grootendorst, R. (2004). A Systematic Theory of Argumentation: The Pragma-Dialectical Approach. Cambridge: Cambridge University Press.

van Eemeren, F. H. (2013). Strategic maneuvering in argumentative discourse in political deliberation. J. Argumentation Context 2, 10-31. doi: 10.1075/ jaic.2.1.01eem

van Eemeren, F. H., and Garssen, B. (2010). "Constraints on political deliberation: European parliamentary debate as an argumentative activity type." in The Functions of Argument and Social Context, 505-514.
Van Ewijk, R., and Sleegers, P. (2010). The effect of peer socioeconomic status on student achievement: a meta-analysis. Educ. Res. Rev. 5, 134-150. doi: 10.1016/j.edurev.2010.02.001

Van Zanten, A. (2003). Middle-class parents and social mix in French urban schools: reproduction and transformation of class relations in education. Int. Stud. Sociol. Educ. 13, 107-123. doi: 10.1080/09620210300200106

Veugelers, W. (2020). How globalisation influences perspectives on citizenship education: from the social and political to the cultural and moral. Compare: J. Comp. Int. Edu. 1-16. [Preprint]. doi: 10.1080/03057925.2020.1716307

Vološinov, V. N. (1986). Marxism and the Philosophy of Language (transl. by L. Matejka and I. I. Titunik). Cambridge, MA: Harvard University Press.

Von Holdt, K. (2014). On violent democracy. Sociol. Rev. 62, 129-151. doi: $10.1111 / 1467-954$ X.12196

Walton, D. (1990). What is reasoning? What is an argument? J. Philos. 87, 399-419. doi: $10.2307 / 2026735$

Walton, D. (2006). How to make and defend a proposal in a deliberation dialogue. Artif. Intell. Law 14, 177-239. doi: 10.1007/s10506-006-9025-x

Wecker, C., and Fischer, F. (2014). Where is the evidence? A meta-analysis on the role of argumentation for the acquisition of domain-specific knowledge in computer-supported collaborative learning. Comput. Educ. 75, 218-228. doi: 10.1016/j.compedu.2014.02.016

Wiggins, D. (1975). Deliberation and practical reason. Proc. Aristot. Soc. 76, 29-51.

Ydesen, C., Acosta, F., Milner, A. L., Ruan, Y., Aderet-German, T., Gomez Caride, E., et al. (2020). Inclusion in testing times - implications for citizenship and participation. Paper commissioned for the UNESCO Futures of Education report.

Yiong-Hwee, T., and Churchill, D. (2007). Using sentence openers to support students' argumentation in an online learning environment. Educ. Media Int. 44, 207-218. doi: 10.1080/09523980701491658

Conflict of Interest: The authors declare that the research was conducted in the absence of any commercial or financial relationships that could be construed as a potential conflict of interest.

Copyright (c) 2021 Larrain, Fortes and Rojas. This is an open-access article distributed under the terms of the Creative Commons Attribution License (CC BY). The use, distribution or reproduction in other forums is permitted, provided the original author(s) and the copyright owner(s) are credited and that the original publication in this journal is cited, in accordance with accepted academic practice. No use, distribution or reproduction is permitted which does not comply with these terms. 
OPEN ACCESS

Edited by:

Sidarta Ribeiro,

Federal University of Rio Grande do

Norte, Brazi

Reviewed by:

Josephine Convertini,

University of Applied Sciences and

Arts of Southern Switzerland

(SUPSI), Switzerland

Marzia Saglietti,

University of Bologna, Italy

*Correspondence:

Carlo Galimbert

carlo.galimberti@unicatt.it

Specialty section:

This article was submitted to

Language Sciences,

a section of the journal

Frontiers in Psychology

Received: 31 January 2021 Accepted: 31 May 2021

Published: 06 July 2021

Citation:

Galimberti C, Bova A, Spanò C and Vergine I (2021) Polydimensional Structure and Psychosocial Functions of the Direct Address in TV Series. Front. Psychol. 12:662215 doi: 10.3389/fpsyg.2021.662215

\section{Polydimensional Structure and Psychosocial Functions of the Direct Address in TV Series}

\author{
Carlo Galimberti*, Antonio Bova, Carmen Spanò and Ilaria Vergine \\ Department of Psychology, Catholic University of the Sacred Heart, Milan, Italy
}

Traditionally, in media studies research, the direct address or aside, i.e., a construction in which a speaker communicates a message directly to the audience breaking the continuity of the narrative flow, has been investigated mainly for its dramaturgical function. The present study aims to consider the direct address as a research object of the social psychology of communication to increase our understanding of this technique by going beyond the analysis of its dramaturgical function. In particular, the direct address will be examined through an integrated approach based on argumentative and conversational tools to highlight its less known polydimensional structure, i.e., diegetic and extra-diegetic dimensions and their interactions, and psychosocial functions, i.e., connecting the characters among each other within the show as well as with the audience. This objective will be achieved by analyzing two different direct addresses from the American TV series House of Cards. The analysis showed that the direct address performs its dramaturgical function by impacting both diegetic and extradiegetic levels. In the first case, as considered in previous studies, these plans are activated in parallel, aiming to build what we have defined as the "strategic subjectivity" of the character who employs this technique. Instead, in the second case-which comprises two direct addresses produced by two different characters - this technique involves the creation of what we will call "platforms of intersubjectivity." In this occurrence, the dramaturgical action establishes a "bridge" between the diegetic and extradiegetic plans that act synergistically. In conclusion, the present study shows how an integrated approach based on argumentative and conversational tools of analysis permits to enlarge the traditional media studies perspective, highlighting the less investigated polydimensional structure and analyzing the psychosocial functions of the direct address, here considered as a research object of the social psychology of communication examined in its diegetic and extra-diegetic dimensions. The integration of the pragma-dialectical approach to argumentation with the interlocutory logic theory has brought to light a new modality of use of the direct address that can be termed "intersubjective aside," a type of aside that can be added to the three already known, i.e., aside ad spectatores, monological aside, and dialogical aside.

Keywords: argumentation, communicative interactions, conversation, direct address, media studies, subjectivity, intersubjectivity, TV series 


\section{INTRODUCTION}

As observed by media researchers (e.g., Marriott, 2007; Gerbaz, 2008; Brown, 2012; Birke and Warhol, 2017), the direct address technique, i.e., a construction in which a speaker communicates a message directly to the audience, breaking the continuity of the narrative flow, is an ideal crossroad for the articulation of the diegetic and extra-diegetic dimensions of a media text. The diegetic dimension is internal to the text's narrative, while the extra-diegetic dimension reaches out to the audience in the context of fruition. Accordingly, the direct address can be described as a monolog that can only be heard by the extradiegetic audience, while the characters standing right next to the speaker are totally unaware of his or her speech. In other words, the distribution of information between characters and viewers is strictly dependent on the privileged relationship between those characters who have access to this technique and the public.

Traditionally, in media studies research, the direct address has been investigated mainly for its dramaturgical function. The present study aims to consider the direct address as a research object of the social psychology of communication, increasing our understanding of the direct address by going beyond the only analysis of its dramaturgical function. The direct address will be examined by adopting an integrated approach based on argumentative (van Eemeren and Grootendorst, 1992; van Eemeren, 2010) and conversational (Trognon and Batt, 2010) tools to highlight its less known polydimensional structure, i.e., diegetic and extra-diegetic dimensions and their interactions, and psychosocial functions, i.e., connecting the characters among each other within the show as well as with the audience. This objective will be achieved by analyzing the direct addresses' use in the American TV series House of Cards (2013-2018). In particular, to highlight the direct address's polydimensional structure and psychosocial functions, we will analyze two different direct addresses. In the first case, as considered in previous studies, e.g., Klarer (2014), the direct address is addressed prevalently to the extra-diegetic dimension. In the second case, instead, the direct address is addressed mainly to the diegetic dimension.

A Netflix Original conceived and produced by Beau Willimon and David Fincher, House of Cards is an adaptation of the homonymous British TV show broadcast by the BBC in 1990 on the novel by Michael Dobbs. The series centers around the ambitious US congressman Francis (Frank) J. Underwood, played by Kevin Spacey, and his equally ambitious wife, Claire Underwood, played by Robin Wright. Frank and Claire Underwood's primary goal in life is to climb the ladder of power up to the top, regardless of what this process might entail. In the construction of its storylines, the direct address stands as the peculiar trait of House of Cards. Within this TV show, its use comes out "to be one of the most conspicuous narrative features of the unfolding episodes" (Klarer, 2014, p. 206), affecting the plot's development, the depiction of characters' profiles, and the audience mode of reception.

In order to present our study, the present paper is structured as follows. In its first part, we will discuss the reasons underlying the growing interest, within media studies, for the investigation of the use of the direct address. Afterward, the object of study and the analytical approach adopted for the analyses will be described, thus providing the methodological and conceptual framework on which the present study is based. In the last part of the paper, the results of the analyses of the two direct addresses considered for this study will be outlines and discussed, followed by a concluding section that summarizes the main findings and comments on their strengths as well as limitations.

\section{ANALYSIS OF THE DIRECT ADDRESS IN MEDIA STUDIES}

In his seminal work, Pfister (1991) analyzes the features of plays in their dramatic and theatrical dimensions, ranging from Greek tragedy and comedy to contemporary theater, with a particular focus on the plays of William Shakespeare. Throughout the body of his exploration, Pfister provides systematic definitions of narrative techniques employed in the construction of representations. Specifically, and of relevance to the study presented here, the author defines the theatrical aside that directly addresses the audience as "aside ad spectatores," or "direct address." This in contrast to, respectively, the "monological aside," i.e., a remark that occurs in dialogue but is not meant to be heard by any of the speaker's interlocutors, and the "dialogical aside," i.e., a remark that is addressed to a specific hearer, but is heard by nobody else present but the intended hearer.

Thus far, most of the studies on the direct address in media objects have focused on the dramaturgical function of the aside ad spectatores, showing how the main function of this technique, through which the continuity of the narrative flow is suddenly broken, is to provide the audience with more background knowledge about a certain situation. For instance, Mittell (2006) highlights how the direct address emerges as a peculiar tool for bolstering narrative complexity as a new form of narrative discursive techniques applied to entertainment television. The diegetic representation constitutes a part of the narration, which is, in fact, stratified since it incorporates and reveals the functioning of the narrative strategies. According to this media scholar, audiences take pleasure in the diegetic twists and the exceptional storytelling techniques needed to pull off such machinations. In this scenario, the educated spectator, who is accustomed to serialized stories with multi-faceted characters, can focus on the diegetic content and the elements that point out its formal construction and reproduction. In recent work, Klarer (2014) focused on the same research object of our study, i.e., the direct addresses the TV series House of Cards, aiming to examine the narrative's effects produced by this technique. The author identifies the primary function of the direct address in the so-called "metalepsis," or narrative transgression: "What makes the aside, both in the theater and in film, equally intriguing is its metaleptic quality" (2014, p. 210). In particular, according to this author, House of Cards' direct address combines two narrative agencies that initially appear to speak in unison-that is, as long as the protagonist seems to be a reliable narrator-but later on 
become discordant, thereby producing a composite format with great narrative potential.

On the other hand, an array of studies shows how the use of the direct address in TV shows can have the function to disclose a character's plans and thoughts and, by doing so, strengthening the connection between the diegetic and extradiegetic dimensions. For instance, Kinney (2019), focusing on the many independent films of the 1960s that feature black actors in moments of direct address, explains how through this technique it was possible to create reciprocity and alignment between the actor and the extra-diegetic audience. In the same vein, Woods (2019) points out how two British television comedies, Chewing Gum and Fleabag, sought to build close connections with their extra-diegetic viewers primarily through the employment of the direct address. In particular, the author shows how, in both comedies, the direct address's use intensifies the embrace of bodily affect and intimate access to interiority, drawing the extra-diegetic audiences to characters' singular perspective, and creating an intensely affective comic intimacy between the protagonists and the extra-diegetic spectatorship. By focusing on the use of the direct address in TV commercials, Hilmes (1985) argues that, like a show, which entails open complicity between spectator and object-seen, the texts, through the direct address, become intermixed and interactive, favoring the spectators' sensation of participation. In addition, the presence of the direct address in the narrative of the storylines elicits fans' engagement and involvement in the possible advancements of a fictional text, as indicated by Walton (1990).

In this section, we have tried to show how, traditionally, in media studies research, the direct address has been investigated mainly for its dramaturgical function. However, in our opinion, the evident dialogical nature of the direct address calls attention to the necessity of looking at the effects produced by the use of this technique as the result of interactional processes occurring on both the diegetic and the extra-diegetic dimensions. And this in order to consider in pragmatic terms its effects on the diegetic and extradiegetic levels and the possible interactions generated between these two levels. To fill this gap in the literature, this work intends to highlight its less explored polydimensional structure and psychosocial functions. In the next section of the paper, the object of study and the analytical approach adopted for the analyses of two different direct addresses from the TV series House of Cards will be described, thus providing the methodological and conceptual framework on which the present study is based.

\section{METHODOLOGY \\ Object of Study}

To highlight the direct address's polydimensional structure and psychosocial functions, we will analyze two different direct addresses from House of Cards. The first example is Frank Underwood's second direct address from the first episode of the first season of the show, and we have named it "Welcome to Washington." In this first example, Frank Underwood's direct address is addressed to the extra-diegetic dimension that reaches out to the audience in the context of fruition. The second example is Claire's direct address from the penultimate episode of the sixth, and last, season of the show, and we have named it "I know you saw it too." In the second case, the direct address is addressed to the diegetic dimension internal to the text's narrative. We have selected for the analysis the above-mentioned direct addresses because they allow us to clearly show the polydimensional structure and psychosocial functions generated by the use of the asides in a TV series, going beyond the so far produced analysis and categorization of its functions. The two sequences were fully transcribed, adopting conversation analysis conventions for transcribing vocal conduct in talk-in-interaction (Sacks et al., 1974; see Appendix). Two researchers revised all transcriptions until a high level of consent (agreement rate $=$ 95\%) has been reached.

\section{Analytical Approach}

The analysis of the two direct addresses considered for this study is based on an integrated analytical approach articulating argumentative and conversational tools of analysis.

In a first phase, to reconstruct the structure of the two direct addresses from an argumentative perspective, in line with other scholars (Kuhn, 1991; van Eemeren and Grootendorst, 2004; Weigand, 2006; Rigotti and Greco Morasso, 2009; Bova, 2019), we will refer to the arguments advanced by a character through the use of a direct address with the scope to support, explain, justify, and defend a standpoint. In this endeavor, the analytical approach for identifying and reconstructing the arguments advanced by a character through the use of a direct address is the pragma-dialectical approach to argumentation (van Eemeren and Grootendorst, 1992; van Eemeren, 2010). According to this approach, the speakers choose the types of arguments that are useful to support their standpoint or weaken the interlocutor's standpoint (van Eemeren and Grootendorst, 1992, p. 138). Using this approach as a guide for the argumentative reconstruction aims to produce an analytic overview of all argumentative components of a discourse, which points are at issue, and which explicit and implicit arguments are advanced. We believe that this model fits particularly this study context because it provides specific criteria for identifying the speaker's standpoint within the direct address and the arguments put forth in support of it.

Subsequently, to reconstruct the two direct addresses' structure from a conversational perspective, we will refer to the interlocutory logic theory (Trognon and Batt, 2010). This approach is a "global theory of the cognitive-affective-social organization of talk-in-interaction" (Trognon and Batt, 2010, p. 19), aiming to describe interlocutory events formally and to build a grammar of the types of dialogue in which we engage and their felicity conditions. Based on the dialogical revision of the Speech Act (Searle and Vanderveken, 1985; Vanderveken, 1990), this approach (Trognon, 2002) is used to explore the mechanisms of the communicative action. More specifically, preparatory conditions and sincerity conditionsbeing two crucial logical components of the illocutionary force (Searle and Vanderveken, 1985, p. 16-19)-will be the focus of our analysis because of their relevance for meaning negotiation process. 
The integration of these two above-mentioned approaches aims to reconstruct the polydimensional structure, i.e., diegetic and extra-diegetic dimensions and their interactions, and psychosocial functions of the characters' direct addresses, i.e., connecting the characters among each other within the show as well as with the audience. We consider the interlocutory logic theory to be the best theoretical frame for analyzing the illocutionary dimension of the direct address, and the illocutionary analysis the ideal "companion" to the pragma-dialectical approach to argumentation. The analysis of direct addresses from this double perspective will give us access to the relational, i.e., illocutionary, and strategic, i.e., argumentative, layers of its discursive plot. Therefore, we will develop sound assumptions on the multiple discursive dimensions of direct address and build a meaningful hypothesis on the psychosocial functions underlying them.

\section{ANALYSIS}

\section{Example 1. "Welcome to Washington"}

Frank Underwood, who had successfully supported the USA president in his election campaign, is passed over as the next Secretary of State. Consequently, he organizes his strategy by putting all of his energy into achieving this goal. In this scene, Frank and Claire Underwood are attending the New Year's gala. Frank Underwood directs his gaze into the camera, thus indicating that this message is meant for an audience outside of the diegesis we are immersed in, explaining his less-than-stellar views on newly elected President Garrett Walker, Vice President of the United States Jim Matthews, and White House Chief of Staff Linda Vasquez. Thanks to Frank Underwood's help, they won the election, and now it's their turn to reward him for it. Frank Underwood is currently the House Majority Whip but is looking for a more prestigious position. Below, we included the full transcription of this sequence.

In this sequence, Frank Underwood's direct address, due to its structure and organization of content, provides the audience with all the main elements characterizing this dialogical technique, from the direct look into the camera to the creation of an alternative space-time frame within the narrative. Frank Underwood's direct gaze into the camera indicates that his monolog is not simply a self-addressed soliloquy, but a carefully crafted speech directed to the viewer outside of the diegetic dimension. Our analysis will initially focus on the direct address' argumentative dimension, and subsequently we will focus on its illocutionary dimension.

Frank Underwood's direct address's argumentative reconstruction shows how he wants to convince the extradiegetic audience, i.e., the people watching the show, that he deserves a more prominent political role than the one he has now. We can describe Frank Underwood's standpoint as follows: "I deserve a prominent political role." We have identified three different types of arguments advanced by Frank Underwood
Example 1 | Welcome to Washington (S01, E01). Participants: Frank Underwood (FU).

\begin{tabular}{|c|c|}
\hline 1. & (voices in the background) \\
\hline 2. FU: & $\begin{array}{l}\text { Oh - President-elect Garrett Walker (he turns } \\
\text { towards the camera, }\end{array}$ \\
\hline 3. & $\begin{array}{l}\text { clapping and indicating the president elect). Do } \\
\text { I like him? No. }\end{array}$ \\
\hline 4. & $\begin{array}{l}\text { Do I believe in him? That's beside the point. } \\
\text { (he says "yes" with }\end{array}$ \\
\hline 5. & $\begin{array}{l}\text { the head) Any politician that gets } 70 \text { million } \\
\text { votes has }\end{array}$ \\
\hline 6. & $\begin{array}{l}\text { tapped into something larger than himself. Larger } \\
\text { than even me, as }\end{array}$ \\
\hline 7. & $\begin{array}{l}\text { much as I hate to admit it. (he begins to walk } \\
\text { and indicates the }\end{array}$ \\
\hline 8. & $\begin{array}{l}\text { president) And look at that winning smile } \\
\text { those, trusting eyes. I }\end{array}$ \\
\hline 9. & $\begin{array}{l}\text { latched onto him early on and made myself vital. } \\
\text { (he stops in }\end{array}$ \\
\hline 10. & $\begin{array}{l}\text { front of the camera) After } 22 \text { years in congress, } \\
\text { (he nods) I can }\end{array}$ \\
\hline 11. & smell which way the wind is blowing. (0.1) \\
\hline 12. & $\begin{array}{l}\text { Oh, - Jim Matthews (he turns himself and } \\
\text { indicates Matthews with }\end{array}$ \\
\hline 13. & $\begin{array}{l}\text { the hand), his right honorable } 10 . \\
\text { vice-president. - Former }\end{array}$ \\
\hline 14. & $\begin{array}{l}\text { governor of Pennsylvania. He did his duty in } \\
\text { delivering the }\end{array}$ \\
\hline 15. & $\begin{array}{l}\text { keystone state, bless his heart, and now they're } \\
\text { about to put him }\end{array}$ \\
\hline 16. & $\begin{array}{l}\text { out to pasture, (he indicates him with the hand). } \\
\text { But he looks }\end{array}$ \\
\hline 17. & $\begin{array}{l}\text { happy enough, doesn't he? (he turns towards the } \\
\text { vice-president and }\end{array}$ \\
\hline 18. & $\begin{array}{l}\text { then, towards the camera) }=(0.2)=\text { For some, - } \\
\text { it's simply the }\end{array}$ \\
\hline 19. & $\begin{array}{l}\text { size of the chair, (he walks again and gets a } \\
\text { glass of wine) }\end{array}$ \\
\hline 20. & $\begin{array}{l}\text { (0.2) Huh. }=(0.2)=\text { Linda Vasquez (he indicates } \\
\text { her with the }\end{array}$ \\
\hline 21. & $\begin{array}{l}\text { hand), - Walker's chief of staff. - I got her } \\
\text { hired. She's a }\end{array}$ \\
\hline 22. & $\begin{array}{l}\text { woman, check, and a latina, check (he indicates } \\
\text { her with the }\end{array}$ \\
\hline 23. & $\begin{array}{l}\text { hand), but more important than that, - she's as } \\
\text { tough as a two- }\end{array}$ \\
\hline 24. & dollar steak. Check, check, check. \\
\hline 25. & (he stops in front of the camera) (2.0) \\
\hline 26. & $\begin{array}{l}\text { When it comes to the White House, you not only } \\
\text { need the keys in }\end{array}$ \\
\hline 27. & $\begin{array}{l}\text { your back pocket, - you need the gatekeeper (he } \\
\text { indicates his }\end{array}$ \\
\hline 28. & $\begin{array}{l}\text { back with the hand) (0.2) (he walks again) } \\
\text { As for me, - I'm just a }\end{array}$ \\
\hline 29. & $\begin{array}{l}\text { lowly house majority whip. - I keep things moving } \\
\text { in a congress }\end{array}$ \\
\hline 30. & $\frac{\text { choked by pettiness and lassitude. }}{\text { clear the pipes }} \underline{\text { My job is to }}$ \\
\hline 31. & $\begin{array}{l}\text { and keep the sludge moving. - But I won't have to } \\
\text { be a plumber }\end{array}$ \\
\hline 32. & much longer. I've done my time \\
\hline 33. & (he points the finger at the camera). \\
\hline 34. & $\frac{\text { I backed the right man }}{\text { stage). }(0.3)}$ (he turns towards the \\
\hline 35. & $\begin{array}{l}\text { (he turns towards the camera) } \\
\text { Give and take. }(0.1)\end{array}$ \\
\hline 36. & $\frac{\text { Welcome to Washington }}{\text { walks away). }}$ \\
\hline
\end{tabular}


to convince this audience that he deserves a more prominent political role.

Frank Underwood's first argument is based on the importance of being ambitious, and he immediately wants the audience to know that he is very much ambitious. After the President-elected Garrett Walker, he introduces Vice President Jim Matthews. Franks Underwood describes him as a politician with no power: (turns 14-17) "He did his duty in delivering the Keystone State, bless his heart. Now they're about to put him out to pasture. But he looks happy enough, doesn't he?". According to Frank Underwood, Vice President Matthews looks happy even if he does not have any power, since (turns 18-19) "for some, it's simply the size of the chair." Frank Underwood tells the audience that the vice-presidency is not enough to give him the power he wants. He does not look for a nice, big chair. He wants the presidency, the real power. We can describe Frank Underwood's first argument by the following sentence: "For some, it's simply the size of the chair."

Frank Underwood's second argument is based on the importance of being an experienced politician. He starts his direct address by introducing the President-elected Garrett Walker and informing the audience that he supported him during the presidential elections. Frank Underwood says that he spent 22 years in Congress, and after all this time, he acquired a crucial political skill: (turns 10-11) "I can smell which way the wind is blowing." It does not matter if he believes in President-elected Walker's qualities or not. According to Frank Underwood, what is important is that he supported the right man. We can describe his second argument by the following sentence: (turn 32) "I've done my time."

Frank Underwood's third argument is based on the importance of controlling people and information in a place like the White House and a city like Washington D.C. After introducing the President-elected Garrett Walker and Vice President Jim Matthews, he introduces a third character, Linda Vasquez, the White House Chief of Staff. Because of her role within the White House, Frank Underwood describes Linda as the person who controls who goes through the President's office. Linda is the White House gatekeeper, and Frank Underwood tells the audience that he has control over the gatekeeper because he (turn 21) "got her hired." We can describe his third argument by the following sentence: (turns 26-27) "When it comes to the White House, you not only need the keys in your back pocket, you need the gatekeeper."

The analytical overview of the argumentative reconstruction of Frank Underwood's direct address's is summarized below:

Issue: Does Frank Underwood deserve a prominent political role? Frank Underwood's standpoint: Yes, I deserve a prominent political role.

Frank Underwood's arguments: (a) For some, it's simply the size of the chair;

(b) I've done my time;

(c) When it comes to the White House, you not only need the keys in your pocket, you need the back gatekeeper.
Turning to the reconstruction of the structure of Frank Underwood's direct address from a conversational perspective, the principal aim of this second phase of analysis is to describe the illocutionary mechanisms on which Frank Underwood's direct address is based. In particular, the analysis will now focus on the illocutionary force of the statements presupposed by the three arguments advanced by Frank Underwood, as identified in the first phase of the analysis.

Based on the dialogical revision of the Speech Act Theory (Searle and Vanderveken, 1985), the three statements presupposed by the three arguments advanced by Frank Underwood constitute the preparatory conditions of what is explicitly affirmed by the three arguments and therefore of the standpoint itself. A preparatory condition is a state of affairs that must be presupposed by the speaker in employing a particular illocutionary force, e.g., promising, advising, warning, asserting, etc., and it is a necessary condition for the non-defective employment of that force. In a real conversation, "in the performance of a speech act, the speaker presupposes the satisfaction of all the preparatory conditions" (Searle and Vanderveken, 1985, p. 17).

In the first example we have analyzed, Frank Underwood, acting in a fictional conversation, needs to explicit the presuppositions useful to build the conversational context with the extra-diegetic audience, i.e., the people watching the show. By doing so, Frank Underwood produces some effects psychosocial in nature. He does so in a 2 -fold manner. First, by sharing his secret goal, i.e., (turns 31-32) I won't have to be a plumber much longer, with the audience, Frank Underwood puts himself on the border that divides the diegetic from the extra-diegetic dimension, accomplishing a double task: (a) at the diegetic level, he contributes to building his subjectivity; (b) at the extradiegetic level, he proposes to the audience the role of the addressee of his asides. Second, by using irony and sarcasm, he exploits Grice's maxim of quality and engages the audience in cooperative actions, establishing illocutionary relationships with strong relational fallouts with the extra-diegetic audience (Vanderveken, 1990, p. 72-75).

A further step in producing some effects psychosocial in nature is performed by Frank Underwood's last remark, (turn 36) "Welcome to Washington." According to Searle and Vanderveken (1985, p. 216), "to welcome somebody is to receive him with hospitality, and thus welcoming might be defined as an expression of pleasure or good feeling about the presence or arrival of someone. Welcoming... is essentially hearerdirected." We believe that Frank Underwood's last remark can be defined as the pragma-semiotic root of this sequence. In fact, by saying "Welcome," Frank Underwood manifests his intention to attribute a dialogical role to the extra-diegetic audience because it becomes an essential partner for the validation of his future direct addresses, leading them on a path that goes from the extra- to the intra-diegetic level. Besides, Frank Underwood also attributes a relational role to the extra-diegetic audience. It becomes a real partner in the narrative process by virtue of a complementary path going from the intra- to the extra-diegetic dimension. In the light of these considerations, we could say that the extra-diegetic audience became a reality composed of "required guests." 
In conclusion, the analysis of Frank Underwood's direct address by adopting an integrated approach based on the pragma-dialectical approach to argumentation and the interlocutory logic theory has shown how, by advancing arguments in support of his standpoint, Frank Underwood gives rise to an effective intertwinement among the actions accomplished by his talk, the discursive world, and the roles he proposes to the audience. The analysis also revealed the central role of the character: he is at the center of the mediation between diegetic and extra-diegetic dimensions that, as we noted earlier, are traditionally distinct. It is precisely on this "centrality" that Frank Underwood builds his "strategic subjectivity," deciding which elements of his identity should come into play. We use the term "strategic" because his actions, on the one hand, aim at building relationships with the other characters and commenting on the events; on the other hand, they guide the audience in understanding the fictional events and orient it toward modes of fruition that respond to the dramaturgical plan.

\section{Example 2. "I Know You Saw It Too"}

In the second scene selected for analysis, Claire Underwoodwho in the sixth season decides to return to her maiden name, Claire Hale - has a sit-down interview on a national TV program, during which she announces that she is pregnant with a little baby girl (turn 8). She also depicts the figure of her dead husband, Frank Underwood, as a horrible person, an unknowable con man (turns 22-23). Doug Stamper, Frank Underwood's closest old friend and collaborator, watches this interview with his arms folded. Claire Underwood, through a dialogic direct address (cf. Pfister, 1991) directed to Doug Stamper, within the same diegetic environment, utters, (turn 23) "Come and get me, Doug." Doug Stamper's undying friendship for Frank Underwood puts him into a fit of rage. At this point, Doug Stamper gets up from his couch, goes into the washroom, and shaves his beard, dramatically cutting off his face. Then, through a direct address directed to the audience in the extra-diegetic context of fruition, he says, (turn 24) "She leaves me no choice." Below, we included the full transcription of this sequence. Claire Underwood's direct address (turn 23) and Doug Stamper's direct address (turn 24) are written in bold:

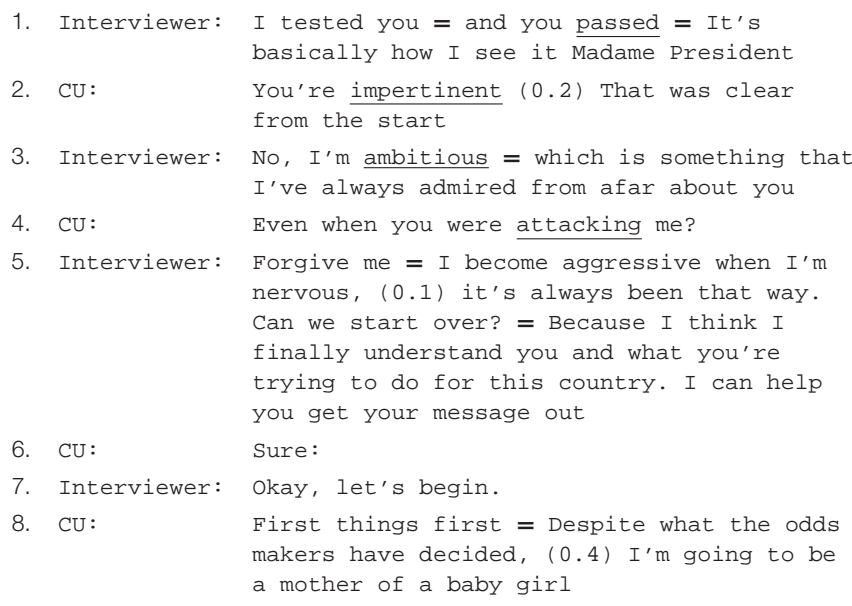

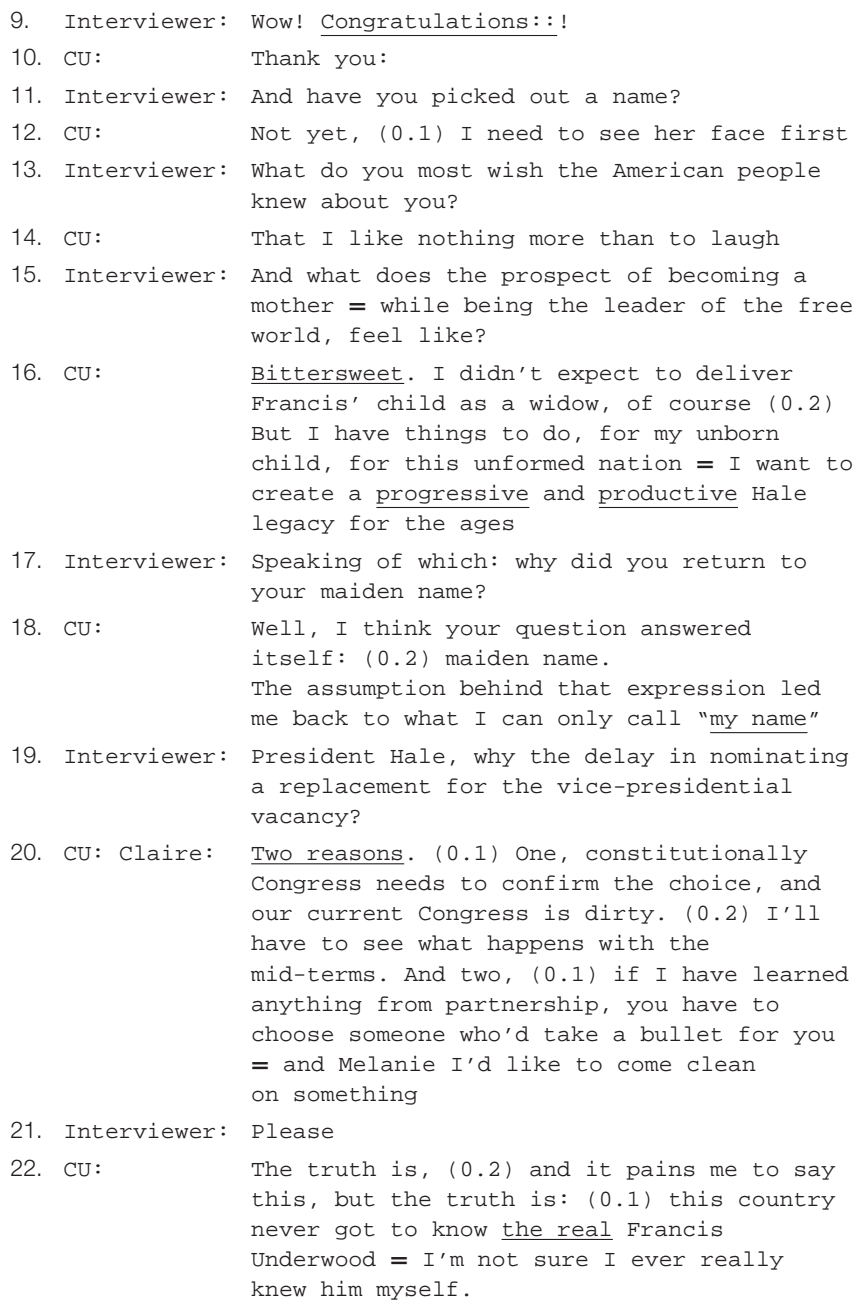

23.

I know you saw it too. He was impossible to know, like all con men. (0.1) He played us all. Come and get me, Doug.

24. DS :

She leaves me no choic

Like the analysis of Example 1, here, too, our analysis will initially focus on the direct address' argumentative dimension, and subsequently we will focus on its illocutionary dimension. In particular, our analysis will be centered on Claire Underwood's direct address directed to Doug Stamper, within the same diegetic environment. This example of aside differs from the previous one for a basic dynamic that emerges as extremely significant. All the previous direct addresses in this TV series were directed to the audience in the extra-diegetic dimension. Instead, in this scene, Clair Underwood's direct address is decidedly unexpected because it is directed to another character, Doug Stamper, within the same diegetic environment, i.e., within the fictional frame of the narrative, and not toward the audience in the extra-diegetic setting. This is the first and only time, in this TV series, that the direct address is employed in this way. The first time that a fictional character addresses another character by adopting a specific modality that pertains to a form of communication 
conceived for bridging two distinct realities, i.e., the diegetic and extra-diegetic dimensions of a media text.

Claire Underwood's direct address's argumentative reconstruction shows how she wants to convince another character, Doug Stamper that Frank Underwood, who was her husband and Doug Stamper's old friend, was betraying everyone, including him. We can describe Claire Underwood's standpoint as follows: "Frank Underwood was cheating us all." We have identified two different types of arguments advanced by Claire Underwood to convince Doug Stamper that Frank Underwood was deceiving all those around him.

Claire Underwood's first argument is based on the fact that she knows that Doug read Frank Underwood's secret diarya collection of thoughts and revelations as expressed in all the direct addresses uttered by Frank Underwood throughout the previous seasons of the series. Therefore, as she does, Doug Stamper is also aware of all the strategic plans and horrible actions carried out by Frank Underwood to reach his own goals. According to Claire Underwood, Doug Stamper cannot deny that Frank Underwood was cheating him too, because she knows that he read Frank Underwood's secret diary. Accordingly, we can describe Claire Underwood's first argument by using the following sentence: "I know you saw his secret diary too."

Claire Underwood's second argument is based on the typical traits characterizing a "con man." Nobody knew that Frank Underwood was cheating everybody because he was a con man and, "like all con men," he was a man impossible to truly know. The fact that both his wife, i.e., Claire Underwood, and his old friend, i.e., Doug Stamper, did not know that Frank Underwood was cheating everybody can be easily explained by the fact that he was a con man, that is, a manipulative individual. Accordingly, we can describe her second argument by using her own words: "He was impossible to know, like all con men."

The analytical overview of the argumentative reconstruction of Claire Underwood's direct address is summarized below:

Issue: Was Frank Underwood cheating us all? Claire Underwood's standpoint: Yes, Frank Underwood was cheating us all.

Claire Underwood's arguments: (a) I know you saw his secret diary too;

(b) He was impossible to know, like all con men.

Turning to the reconstruction of the structure of Claire Underwood's direct address from a conversational perspective, the principal aim of this second phase of analysis is to describe the illocutionary mechanisms on which Claire Underwood's direct address and Doug's successive one are based. In particular, the analysis will now focus on the illocutionary force of $23 \mathrm{CU}$ and $24 \mathrm{D}$, that is the statements effectively uttered by the authors of the two direct addresses.

By adopting a psychosocial stance, we will pay attention to the conversational context of the two direct addresses, that is, to the twenty-two previous speech turns that we will consider with regards to both the speech act's propositional contents and the illocutionary force that appear in them. The consideration of Claire's speech turns from the point of view of propositional contents reveals the emergence of two lines of discourse: one linked to contents of private nature (I have things to do, for my unborn child; the choice of the maiden name), the other of an institutional nature (Hale legacy; I have things to do... for this unformed Nation; replacement of the vice-president).

The convergence of the two lines of discourse (13. I: What do you most wish the American people knew about you? 14. CU: That I like nothing more than to laugh; 15. I: "And what does the prospect of becoming a mother = while being the leader of the free world, feel like?"; 16. CU: "Bittersweet ... But I have things to do, for my unborn child, for this unformed nation") generates Claire's action program that combines the dimension of the attention to desire (unborn child) with that of the exercise of power (unformed Nation). In the commissive with which 16. CU concludes her speech turn ("I want to create a progressive and productive Hale legacy for the ages"), the action program concentrates into a declaration of commitment that synthesizes, in narcissistic terms, the private side (i.e., the exaltation of desire) and the public side (i.e., the exercise of power) that have characterized the entire progression of the interview.

The "turning point" represented by the convergence of the two lines of content also plays a fundamental role for the analysis of the illocutionary dimension. In particular, we refer to the "revelation" of the truth (23. CU: "The truth is,...") regarding the "real" nature of Frank Underwood. This revelation completes the cognitive environment's construction that frames the two direct addresses that conclude the sequence.

Let us now consider the first direct address, 23. CU, made up of four speech acts, three assertive and one directive.

(a1) "I know you saw it too": assertive that expresses a belief.

(a2) "He was impossible to know, like all con men": assertive that express a judgment.

(a3) "He played us all": assertive that expresses a judgment.

(d1) "Come and get me, Doug": directive expressed by an imperative but that, as we will point out shortly, also represents a desire (Condoravdi and Lauer, 2012, p. 39).

According to the illocutionary analysis suggested by Beversluis (1971, p. 347) in some cases "I know that p" can be considered in the same way as "I warn you that p." As it is also well-known, thanks to the semantic analysis of the verb "to warn" (Searle and Vanderveken, 1985), "I warn you that P" from the illocutionary point of view has a double nature and can be considered as either directive or assertive "about the state of affairs represented by P." "I can warn (...) you that such and such is the case or I can warn (...) you to do something. But the two uses are not independent. When I warn (...) you that something is the case I am normally warning you that it is the case with a view to getting you to do something about it" (p. 202-203). In our opinion, this is precisely the case represented by the direct address made by CU to Doug. If we consider (turn 23) "I know you saw it too" as equivalent to "I warn you that I know you saw it too," we can see that the speech acts which are part of the same speech turn concretize both the assertive component (turn 23 "He was impossible to know, like all con men. He played us all") and the directive component (turn 23 "Come and get me, Doug"), following the binary structure that, as we have seen in carrying out the analysis of the propositional component, characterizes the entire sequence. 
To complete the analysis of 23 . CU, we should note that this first direct address remains within the perimeter of the diegesis. In other words, we can say that this direct address represents an invitation to construct an intra-diegetic intersubjective context, in the sense that Claire addresses Doug by exiting the conversation with the interviewer while remaining within the narrative of the story. Before proceeding with the analysis of 24. DS, the second direct address of the sequence under consideration, we would like to clarify the meaning adopt for the concept of intersubjectivity. For us, intersubjectivity is the phenomenological bridge between the subject and the other(s). It refers to "the process that allows actors to create a shared world within which they can interact with a good level of inter-comprehension, that is of mutual intelligibility of their communicative intentions" (Galimberti and Spanò, 2017, p. 196). The pillars of such a world are four specific properties that comprise modalities of action and, at the same time, results of the actors' actions.

The first characteristic of a shared world is "the construction of the actors' subjectivities and their mutual recognition by actors themselves." The second one is "the conjoint definition of rules regarding interaction management and the relationships between actors according to situations." The third feature is "the definition of the objects involved in the interaction" and, lastly, the fourth one derives from "the combination of conversational and discursive rules that allows actors to talk about the objects." "These four characteristics demonstrate that co-referring to a shared world is a signal of a back reference to the actors themselves, that is: when actors talk about objects present within their world, they also give information about themselves, leaving clues, signs of subjectivity that are being elaborated during interaction" (Galimberti and Spanò, 2017, p. 196).

If we consider the speech act contained in 24. DS ("She leaves me no choice"), we can see that Doug makes Claire's directive (turn 23 "Come and get me, Doug") successful and satisfied (Vanderveken, 1990, p. 129-134). This entails two consequences: (a) Doug accepts to enter the "intersubjective intra-diegetic platform" that Claire has built for him; (b) Doug relaunches to the audience the accepted contents (i.e., Claire's project of power and desire) making explicit his being "caught" in this game and shaping them into a new "intersubjective platform," this time of extra-diegetic nature.

In summary, then, we can schematize the results of our analysis as follows: Direct address $1=$ diegetic dimension $=$ creation of an intersubjective context, of an "intersubjective platform (between Claire and Doug) of intra-diegetic nature." Direct address 2 = extra-diegetic dimension = Doug accepts Claire's proposal, enters the "intersubjective intra-diegetic platform," confirms its contents and commits himself to their realization. By addressing the audience, he re-launches Claire's proposal to the viewers, making it the content of a new "intersubjective platform," this time of extra-diegetic nature.

Concluding remarks: (a) desire and power are the elements that allow to "manage" the simulations and manipulations of a "con man"; (b) the two direct addresses open and close the circle between "inside" and "outside" the narration, establishing a new modality of use of the direct address that, in our opinion, can be termed "intersubjective aside," a mode that can be added to the three known and already described at the beginning of this work, that is: aside ad spectatores, monological aside, and dialogical aside.

\section{DISCUSSION}

The direct address constitutes one of the most interesting dramaturgical techniques used in performative arts. Traditionally, in media studies research, we have seen that the direct address has been investigated mainly for its dramaturgical function (e.g., Hilmes, 1985; Mittell, 2006; Klarer, 2014; Kinney, 2019; Woods, 2019). By adopting an integrated approach based on the pragma-dialectical approach to argumentation (van Eemeren and Grootendorst, 1992; van Eemeren, 2010) and the interlocutory logic theory (Trognon and Batt, 2010), the present study considered the direct address as a research object of the social psychology of communication and aimed to highlight its less known polydimensional structure and psychosocial functions. In this endeavor, we analyzed two different direct addresses from the American TV series House of Cards. In the first case, the direct address is addressed to the extra-diegetic dimension that reaches out to the audience in the context of fruition (cf. aside ad spectatores, in Pfister, 1991). In the second case, instead, the direct address is addressed to the diegetic dimension internal to the text's narrative (cf. dialogical aside, in Pfister, 1991). At this juncture, it seems appropriate to take stock of some findings of our study.

In line with previous studies, the findings of the analysis of the two examples of direct addresses show that the direct address not only permits the connection between diegetic and extra-diegetic dimension, but it also has the function of empowering the diegetic dimension by modifying the text's narrative. Considering the effects of the direct address on the extra-diegetic dimension, we showed how its use permits disseminating information to the audience, contributing to creating a frame of expression only for the characters and the audience. Through the analysis of the first example, we have seen how Frank Underwood distances himself from the world depicted on the screen and moves toward the world on the other side, and his direct address exemplifies the constant blurring of diegetic and extra-diegetic dimensions (Tindale, 2015). In this case, the direct address is employed as a strategic maneuvering (van Eemeren and Houtlosser, 2002) by Frank Underwood to persuade the extra-diegetic audience that he deserves a more prominent political role than the one he has till that moment. The reasons supporting his argumentative message relied on his personality and not, instead, on his political ideas' virtues. It is all about his personality, his skills, and his strengths, while there is no mention of what he wants to realize for his country. It's all about his personality, not about politics. In addition, the findings of the analysis of the two examples of direct addresses also bring to light that when the direct address is addressed to the extra-diegetic dimension that reaches out to the audience in the context of fruition, it conveys the characters' subjectivities to the audience.

As far as the diegetic dimension is concerned, in line with previous studies (e.g., Mittell, 2006; Klarer, 2014), we lit up how the direct address has the function of empowering the 
diegetic dimension by modifying the text's narrative. In the second example analysis, we have seen that Claire Underwood's direct address differs from the previous one because it is directed to another character, Doug Stamper, within the same diegetic environment, i.e., within the fictional frame of the narrative, and not the extra-diegetic audience. In particular, in this case, the direct address is employed as a strategic maneuvering (van Eemeren and Houtlosser, 2002) by Claire Underwood to convince another character, Doug Stamper, that Frank Underwood, who was considered by Doug Stamper as a real friend, was cheating everyone, including him. Accordingly, the direct address is employed to modify the text's narrative and not, as commonly used, connect diegetic and extradiegetic dimensions.

Moreover, the analysis findings have also brought to light that when the direct address is used creatively, as in the second example, it gives rise to surprising effects on intersubjectivity. The building and sharing of what we have called "intersubjective platform of intra-diegetic nature" and "intersubjective intra-diegetic platform" is a clear example of what such a dramaturgical technique can bring about to develop the story and fill up the divide with the audience when strategically used.

The polydimensional analytical approach used in this study has shown that the direct address technique gives birth to what we could call a "mediated conversation," a specific place where diegetic and extra-diegetic dimensions in TV series are articulated. The integration of the pragma-dialectical approach to argumentation with the interlocutory logic theory allowed us to see from different angles the intertwine of argumentative strategies and illocutionary characterization. The link between argumentative analysis and illocutionary analysis is here considered from the point of view of the role played by preparatory conditions and sincerity conditions on the production and organization of arguments in direct address. The analysis of the illocutionary layer of the two examples has shown the importance of these two family of conditions. The point of articulation between argumentative analysis and illocutionary analysis is constituted by the arguments, which constitute the preparatory conditions for Frank and Claire Underwood's speech acts. In our opinion, if we do not take into consideration these two elements brought to light in the analysis of the illocutionary layer, we cannot understand why Frank Underwood, in the first example, and Claire Underwood, in the second example, advance the arguments that we have reconstructed through argumentative analysis.

\section{REFERENCES}

Beversluis, J. (1971). "I Know": an illocutionary analysis. Southern J. Philos. 9, 345-351. doi: 10.1111/j.2041-6962.1971.tb02147.x

Birke, D., and Warhol, R. (2017). "Multimodal you: playing with direct address in contemporary narrative television," in How to Do Things with Narrative: Cognitive and Diachronic Perspectives, eds J. Alber and G. Olson (Berlin, Boston, MA: De Gruyter), 141-156. doi: 10.1515/9783110569 957-010

\section{CONCLUSIONS}

In conclusion, the present study shows how an integrated approach based on argumentative and conversational tools of analysis permits to enlarge the traditional media studies perspective, highlighting the less known polydimensional structure and analyzing the psychosocial functions of the direct address, here considered as a research object of the social psychology of communication examined in its diegetic and extradiegetic dimensions. The integration of argumentation theory and illocutionary analysis is a clear gain for researchers who are interested in studying media also from an "outer" perspective, not bounding themselves to the "media text," but opening themselves to the "media context," that is the social scene on which nowadays - via the social media-an essential part of the fortune of tv series is determined. Through the integration of the pragma-dialectical approach to argumentation (van Eemeren and Grootendorst, 1992; van Eemeren, 2010) with the interlocutory logic theory (Trognon and Batt, 2010), we have shown that the two direct addresses analyzed open and close the circle between "inside" and "outside" the narration, establishing a new modality of use of the direct address that, in our opinion, can be termed "intersubjective aside," a type of aside that can be added to the three known and already described at the beginning of this work, i.e., aside ad spectatores, monological aside, and dialogical aside.

This study constitutes the first step toward a further articulation of the argumentative and conversational tools of analysis. Their improved integration will allow us to work on a wide range of research problems traditionally studied in many different scientific fields. This endeavor would have a 2 -fold objective: at the level of data production, it is to improve the definition of the research objects to be investigated; at the level of data analysis, it is to adopt analytical tools able to increase our understanding of the complexity of our research objects from an integrated argumentative and conversational perspective.

\section{DATA AVAILABILITY STATEMENT}

The original contributions presented in the study are included in the article/supplementary material, further inquiries can be directed to the corresponding author/s.

\section{AUTHOR CONTRIBUTIONS}

All authors listed have made a substantial, direct and intellectual contribution to the work, and approved it for publication.

Bova, A. (2019). Dialogical construction of parental feeding strategies during family mealtimes. J. Health Psychol. doi: 10.1177/1359105319884600

Brown, T. (2012). Breaking the Fourth Wall: Direct Address in the Cinema. Edinburgh: Edinburgh University Press.

Condoravdi, C., and Lauer, S. (2012). Imperatives: meaning and illocutionary force. Empirical Issues Syntax Seman. 9, 37-58.

Galimberti, C., and Spanò, C. (2017). Intersubjectivity in media consumption as a result of the relation between texts and contexts: The cases of Game of Thrones. Essais 12, 191-208. doi: 10.4000/essais.3091 
Gerbaz, A. (2008). Direct Address, ethical imagination, and errol morris's interrotron. Film Philos. 12, 17-29. doi: 10.3366/film.2008.0013

Hilmes, M. (1985). The television apparatus: direct address. Jo. Film Video $37,27-36$.

Kinney, K. (2019). Facing the camera: black actors and direct address in independent films of the 1960s. J. Cine. Media Stud. 59, 66-88. doi: $10.1353 /$ cj.2019.0063

Klarer, M. (2014). Putting television 'aside': novel narration in house of cards. New Rev. Film Televis. Stud. 12, 203-220. doi: 10.1080/17400309.2014.885818

Kuhn, D. (1991). The Skills of Argument. New York, NY: Cambridge University Press. doi: 10.1017/CBO9780511571350

Marriott, S. (2007). Live Television: Time, Space and the Broadcast Event. London: Sage Publications.

Mittell, J. (2006). Narrative complexity in contemporary american television. Velvet Light Trap 58, 29-40. doi: 10.1353/vlt.2006.0032

Pfister, M. (1991). The Theory and Analysis of Drama. Cambridge: Cambridge University Press.

Rigotti, E., and Greco Morasso, S. (2009). "Argumentation as an object of interest and as a social and cultural resource," in Argumentation and Education: Theoretical Foundations and Practices, eds N. Muller Mirza and A. N. PerretClermont (New York, NY: Springer), 9-66. doi: 10.1007/978-0-387-98125-3_2

Sacks, H., Schegloff, E. A., and Jefferson, G. (1974). A simplest systematics for the organisation of turn-taking for conversation. Language 50, 696-735. doi: 10.1353/lan.1974.0010

Searle, J., and Vanderveken, D. (1985). Foundations of Illocutionary Logic. Cambridge: Cambridge University Press. doi: 10.1007/1-4020-3167-X_5

Tindale, C. W. (2015). The Philosophy of Argument and Audience Reception. Cambridge: Cambridge University Press. doi: 10.1017/CBO9781316 181645

Trognon, A. (2002). "Speech acts and the logic of mutual understanding," in Essays in Speech Acts Theory, eds D. Vanderveken and S. Kubo (Amsterdam: John Benjamins and Sons), 121-133. doi: 10.1075/pbns.77.08tro

Trognon, A., and Batt,. M. (2010). "Interlocutory logic. A unified framework for studying conversational interaction," in New Adventures in Language and Interaction, ed J. Streeck (Amsterdam: John Benjamins), 9-46. doi: 10.1075/pbns.196.02tro

van Eemeren, F. H. (2010). Strategic Maneuvering in Argumentative Discourse. Amsterdam, Philadelphia, PA: John Benjamins doi: 10.1075/aic.2

van Eemeren, F. H., and Grootendorst, R. (1992). Argumentation, Communication, and Fallacies. A Pragma-Dialectical Perspective. Hillsdale, NJ: Erlbaum.

van Eemeren, F. H., and Grootendorst, R. (2004). A Systematic Theory of Argumentation: The Pragma-dialectical Approach. Cambridge: Cambridge University Press. doi: 10.1017/CBO9780511616389

van Eemeren, F. H., and Houtlosser, P. (2002). "Strategic manoeuvring: Maintaining a delicate balance," in Dialectic and Rhetoric: The Warp and Woof of Argumentation Analysis, eds F. H. van Eemeren and P. Houtlosser (Dordrecht: Kluwer), 131-159. doi: 10.1007/978-94-015-9948-1_10

Vanderveken, D. (1990). Meaning and Speech Acts: Volume 1, Principles of Language Use. New York, NY: Cambridge University Press.

Walton, K. L. (1990). Mimesis as Make-believe: On the Foundations of the Representational Arts. Cambridge, London: Harvard University Press.

Weigand, E. (2006). Argumentation: the mixed game. Argumentation 20, 59-87. doi: $10.1007 / \mathrm{s} 10503-006-9000-4$

Woods, F. (2019). Too close for comfort: direct address and the affective pull of the confessional comic woman in chewing gum and fleabag. Communic. Cult. Crit. 12, 194-212. doi: $10.1093 / \mathrm{ccc} / \mathrm{tcz} 014$

Conflict of Interest: The authors declare that the research was conducted in the absence of any commercial or financial relationships that could be construed as a potential conflict of interest.

Copyright (c) 2021 Galimberti, Bova, Spanò and Vergine. This is an open-access article distributed under the terms of the Creative Commons Attribution License (CC $B Y)$. The use, distribution or reproduction in other forums is permitted, provided the original author(s) and the copyright owner(s) are credited and that the original publication in this journal is cited, in accordance with accepted academic practice. No use, distribution or reproduction is permitted which does not comply with these terms. 


\section{APPENDIX}

\section{Transcriptions Conventions}

- cut off of the prior word or sound

( ) description of situation/speaker's actions

word forms of stressing (pitch and/or volume)

(0.1) elapsed time in tenths of seconds

$=$ lack of interval between the end of a prior and start of a next piece of talk

(h) explosive aspiration

${ }^{\circ}$ ( ) low in volume

: prolonging of sounds

// // segments overlapped by the talk of another 


\section{OPEN ACCESS}

Edited by:

Cheryl J. Craig,

Texas A\&M University, United States

Reviewed by:

Janet Hoskin,

University of East London,

United Kingdom

Eliza Pinnegar,

Independent Researcher,

Orem, United States

Federico Manzi,

Catholic University of the

Sacred Heart, Italy

*Correspondence:

Äli Leijen

ali.leijen@ut.e

Specialty section:

This article was submitted to Educational Psychology, a section of the journal

Frontiers in Psychology

Received: 04 January 2021

Accepted: 16 August 2021 Published: 10 September 2021

Citation:

Leijen Ä, Arcidiacono F and Baucal A (2021) The Dilemma of Inclusive Education: Inclusion for

Some or Inclusion for All.

Front. Psychol. 12:633066.

doi: 10.3389/fpsyg.2021.633066

\section{The Dilemma of Inclusive Education: Inclusion for Some or Inclusion for All}

\author{
Äli Leijen ${ }^{1 *}$, Francesco Arcidiacono ${ }^{2}$ and Aleksandar Baucal ${ }^{3}$ \\ IInstitute of Education, University of Tartu, Tartu, Estonia, ${ }^{2}$ Research Department, Haute Ecole Pédagogique BEJUNE, \\ Biel/Bienne, Switzerland, ${ }^{3}$ Faculty of Philosophy, University of Belgrade, Belgrade, Serbia
}

In this paper, we intend to consider different understandings of inclusive education that frame current public and professional debates as well as policies and practices. We analyze two - somewhat opposing - discourses regarding inclusive education, namely, the "inclusion for some" - which represents the idea that children with special needs have a right to the highest quality education which can be delivered by specially trained staff, and the "inclusion for all" - which represents the idea that all children regarding their diverse needs should have the opportunity to learn together. To put the two discourses in a dialogical relation, we have reconstructed the inferential configurations of the arguments of each narrative to identify how the two definitions contribute to position children with and without special needs and their teachers. The results show the possibilities to bridge the two narratives, with respect to the voices they promote or silence, the power relations they constitute, and the values and practices they enact or prevent.

Keywords: inclusive education, special education, public discourse, argumentum model of topics, dialogue

\section{INTRODUCTION}

Inspired by social justice ideas, the Convention on the Rights of the Child (UN, 1989) and the Salamanca Statement (UNESCO, 1994), many European countries have developed policies and implemented practices to promote inclusive education (Arcidiacono and Baucal, 2020; Nelis and Pedaste, 2020). Consequently, more children with special education needs ${ }^{1}$ are nowadays learning with their peers in mainstream schools and the number of special schools has decreased. Although this is a trend in different countries in Europe and in the Global North, there are several challenges. Most notably, there is still no clear understanding of inclusive education. Researchers, policy makers, and teacher educators have diverse understandings (Haug, 2017; Van Mieghem et al., 2018; Kivirand et al., 2020), which range from the idea that special education is itself a form of inclusive education, to the observation that all children are, for the majority, learning together in an inclusive setting (Ainscow and Miles, 2008; Hornby, 2015; Kivirand et al., 2020). Magnússon (2019) has concluded that the "implementations, interpretations

${ }^{1}$ In line with OECD definition, in this paper, we refer to "children with special education needs" as an umbrella term indicating children who require additional resources or accommodating dominant educational practices in order to ensure equal learning opportunities to them. The term refers to children with disabilities, children with learning difficulties, and children living in disadvantage conditions (OECD, 2007). 
and definitions of the concept vary greatly both in research and in practice, between countries and even within them" (ibid, p. 678).

These different discourses are present in several societies, but the debates are more heated in contexts which more recently have started to implement inclusive education practices, such as Eastern Europe and former Soviet countries (Florian and Becirevic, 2011; Stepaniuk, 2019). One of the reasons for so many challenges in the latter context is the past experience of a strongly segregated educational system. This historical context is illuminated in the views of teachers, parents, and the general public.

In this paper, we will analyze two - somewhat opposing - discourses regarding inclusive education encapsulating two positions that are in the core of many current debates about inclusive education. The first one ("inclusion for some") represents the idea that children with special needs have a right to highest quality education which can be best delivered by specially trained staff in a specialized and often segregated environment, while the second one ("inclusion for all") represents the idea that all children regarding their diverse needs should have the equal opportunity to learn together in a regular education setting.

In this paper, we are going to put the two discourses in a dialogical relation. Through an argumentative analysis based on the reconstruction of the inferential configurations of arguments, we intend to identify how the two definitions contribute to position children (with and without special needs) and teachers, whose voices they promote and whose voices are silenced, what power relations they constitute, and what values and practices they enact or prevent. The possibility to map out the reasoning beyond these arguments is discussed as the starting point for bridging the existing conceptions about inclusive education. Prior to introducing the two narratives, we introduce briefly the background of inclusive education in Estonia which forms the context of the current study.

\section{INCLUSIVE EDUCATION IN ESTONIA}

Similarly to many Eastern European countries, Estonia has a long special education tradition, which is influencing acceptance of the principles and the actual practices of inclusive education. These principles have been established at the legislative level in Estonia since 2010, most notably the law states that students with special needs have the right for studying in their schools of residence with their peers (Basic Schools and Upper Secondary Schools Act, 2010, 2019). In accord with the changes in the legislative framework, the number of pupils with special educational needs in mainstream schools has increased; however, another phenomenon has appeared - the number of students enrolled in special classes in mainstream schools has also increased (Räis et al., 2016). These special classes are often taught by teachers of special education and not by regular teachers. Although many school leaders understand the need for inclusive education, their main concern is a lack of availability of support specialists - including special needs teachers, speech therapists, and phycologists (Räis and Sõmer, 2016). Although the expertise of support specialists is highly valued in Estonian schools and kindergartens, more and more teachers have recognized the importance of their own professional development related to supporting diverse learners. For example, the comparison of TALIS 2013 and TALIS 2018 survey data (OECD, 2020) showed that teachers' participation in professional development activities related to supporting diverse learners has significantly increased in Estonia and at the same time teachers indicated that training in this area is for them still the largest need for professional development. Consequently, diverse in-service training courses are available for teachers. An analysis of the course content at one of the major universities in Estonia providing teacher education showed that the core content of these courses has tended to focus on didactical methods of teaching students with special educational needs rather than on strategies of inclusive pedagogy. However, more recent in-service courses have emphasized social justice, possibilities for participation, and inclusive pedagogies as well (e.g., Kivirand et al., 2021). This brief overview illuminates that very different perspectives and practices are present in Estonia. We will explore these in more detail in the next sections.

\section{TWO DISCOURSES OF INCLUSIVE EDUCATION}

\section{Inclusion for Some}

There have been several articles published in 2020 in Estonian national newspapers arguing that inclusive education is a dream or ideology that does not take into account actual circumstances of reality. In one of such articles (Ehala, 2020), a university professor, who regularly writes about education, cites a recent study conducted in Estonia on the added value of education on children's cognitive abilities. The study showed that $80 \%$ of the children's knowledge and skills can be explained by individual abilities and home background, and only $20 \%$ by the influence of school. The professor argued that children with physical disabilities could be included, but it is problematic to include children who have been raised according to very different principles or who have significant cognitive disabilities. He specified that inclusive education would only be possible in societies which are very homogeneous, most importantly regarding child raising practices and family values. This would result in a situation where there are few differences between children's behaviors and are used for similar norms and regulations. He pointed out as: "Inclusive education is a mirage created by our sense of justice, but its implementation puts young people in a learning environment that is not in line with their home preparation and developmental needs. They are just too special and different so that everyone could learn together in a way that no one suffers." He concluded that we simply need different kinds of environments for different children.

Many of these ideas are also pointed out by some teachers. In 2019, a new educational strategy was prepared for Estonia and in this process, several meetings were held in different 
places across the country. Many teachers were critical regarding the recent policy reform related to inclusive education. On the one hand, teachers are concerned about the learning process and outcomes of the regular children and, on the other hand, their own preparation to support students with special needs. Working with special needs students requires specialized knowledge and expert skills, which many teachers simply do not have. Similar to these views, a group of master students wrote an article in a national newspaper in June 2020 (Kupper et al., 2020) where they stated that although they support the idea of inclusive education, it is only justified if it is carefully organized and sufficient support is available. They also added that inclusive education is certainly not suitable for students with more severe special needs. They point out as: "Inclusion may not be effective in case the teacher does not receive enough support and guidance regarding how to work with a special needs student and the rest of the class at the same time. If, figuratively speaking, the teacher's strength does not overcome the situation, then the increase in behavioral problems, drop-out rates and developmental delays are real dangers."

Moreover, this article also shed light into the perspective of parents of special needs students. They argued as: "A familiar and close-to-home school with a teacher assistant or support specialist does not outweigh the assurance that the child's safety and well-being is guaranteed throughout the day and is cared for by a sufficient number of professionals." Moreover, "Studying at a school close to home may not always be possible if the child needs a much more complex service due to his or her situation, including, for example, special therapies and additional activities. If such a solution is not offered during the school day, parents must find the time and opportunity, usually at the expense of working hours, to provide the necessary service to the child. Thus, the difficulty of the whole situation lies with the parents, who, despite the child's special needs, must be able to maintain optimism, offer equal care and love to the other children of the family in other words, try to live as normal a life as possible while maintaining the ability to work, good relations with the employer and income and one's own emotional balance."

In brief, all these perspectives argue that the development of different students will benefit from specialized learning environments and special teachers who have good expert knowledge and skills for preparing specific educational experiences for maximizing each student's individual potential. Similar viewpoints have also been presented in the international literature: for example, Kauffman and Hornby (2020) criticized inclusive education ideology and leading scholars in the field for the unrealistic claims regarding its implementation and outcomes. They concluded as: "Appropriate instruction is by far the most important task of education for all students, including those with disabilities. Making appropriate instruction a reality for all students requires special education, including teachers with special training, rather than a generic, 'one size fits all' or all-purpose preparation" (p. 10).

\section{Inclusion for All}

In contrast to voices arguing for creating different learning environments for different children, scholars, policy makers, teachers, and parents in favor of inclusion for all stress, in different talks and articles, that all children in a society should have an equal right to get adequate opportunities to develop wellbeing, agency, identities, and competences in order to become capable to participate fully and equally in the society (UNESCO, 1994; Ainscow and César, 2006; Cigman, 2007; Felder, 2019). This objective cannot be reached if some children are educated in a segregated context.

Inspired by social constructivist approaches to learning, teacher educators supporting inclusive education argue that child development depends not only on inherited capacities, but it is also constructed by shared social values, access to educational institutions, technologies (including assistive technologies), and other relevant social resources as well as quality of support provided to the child and opportunities to participate fully and equally in a community.

Teacher educators and policy makers would agree that it is true that current educational systems (schools, teachers, initial education of teachers, practices, technologies, teaching and learning materials, etc.) in many countries have been established based on an assumption that "regular" education, schools, and teachers should work only with "typical" children and other children need to be educated in a specially designed and segregated environment, that is, "special" education (Carrington, 1999; Croll and Moses, 2000; Dyson et al., 2002; Radó et al., 2016; Zgaga, 2019; Koutsouris et al., 2020). However, they would argue that in such an environment, children cannot develop a sense of belonging nor can become full members of the society because of marginalized status and limited opportunities to grow with others (Freeman and Alkin, 2000; Farrell, 2010; Koller et al., 2018). Moreover, in a special education, setting relationships, practices, and technologies tend to be adapted to their constraints instead of being designed to enable children to fully participate in education and society in spite of constraints. Similarly, parents, teachers, and kindergarten/school leaders favoring inclusive education in Estonia would argue for social justice ideas: the importance of growing up within the community and learning at a kindergarten/school close to their home. A father of a child with speech difficulties, who was contacted by an author of this article and asked why he favored his child attending a regular kindergarten instead of a specialized kindergarten, pointed out as: "I can't distinguish my child, who has special (or rather specific) needs, from any other child. How can I agree with her being placed in a school which labels her directly and indirectly as a person who does not fit the norm? Especially when attending kindergarten, she is as special and as normal as every other child who she plays with and a child who plays with her. This should be the norm for any healthy development of a child." Similarly, a teacher and master student (Konetski-Ramul, 2021) and a head of support specialists services (Labi, 2019) have argued for inclusive education in articles published in the national teachers' newspaper in Estonia. In these articles, the authors urged for not separating students 
with special needs easily to special classes or special schools, e.g., Labi (2019) pointed out as: "If today we separate one quarter of children for fear that their involvement could negatively affect the well-being of the other three quarters of children, then as adults there are people in the labor market, in families, or even in the queue at the store, who cannot cope with each other. It is more sustainable to grow together, learn from each other and cope with each other throughout the school journey." Many parents of special needs children would also argue that the most important goal for them is for their children to adapt to society and learn to live with other people. To illustrate this idea, a mother of a young child with multiple disabilities pointed out during a public speech in Estonia that her family's "goal is to support him so he would become a taxpayer."

In order to have an equal opportunity, all children need to be educated in regular education that have conditions, capacities, and resources to be able to adapt to the children needs, capacities, and constraints. Following this, in a case when a school, teachers, discourses, practices, and technologies are not aligned with the needs of some students, it cannot be an acceptable reason for the exclusion of the child, but for adapting the education to the child and his/her learning and developmental needs (Farrell, 2010; Arcidiacono and Baucal, 2020).

The majority of Estonian teachers has adopted learnercentered views about education as reported in international comparison studies, such as TALIS 2013, 2018, and a smaller group has also learned to implement these in practice (many Estonian teachers are still rather traditional and subject-oriented in their teaching practice; OECD, 2014, 2020; see also Leijen and Pedaste, 2018). Teachers who have accepted the childcentered view might not consider a class as a unified mass, instead they might perceive children anyway as special and different, notice variety, individual differences and adapt their teaching accordingly (Breeman et al., 2015). Following, adapting their teaching for a child with special needs would not be so different from any other adaptation of teaching for the child's needs and interests. While discussing the possible challenges of inclusive education during an in-service course taught by the first author of the paper in autumn 2019, a teacher pointed out that "it is very interesting and positively challenging to teach a group of students with a large variety. These are (my) favorite classes." This indicates that teachers might find diversity and variety enriching for themselves as professionals.

\section{GOAL OF THE PAPER}

The aim of this paper is to show, through the conceptual analysis of the two above-mentioned discourses, that it is possible to put these two narratives in a dialogical relation to identify their contribution to position children (with and without special needs) and teachers with respect to the voices they promote or silence about inclusive education, the power relations they constitute, and the values and practices they enact or prevent.

\section{MATERIALS AND METHODS}

We propose an analytical approach based on the argumentum model of topics (AMT) that aims at systematically reconstructing the inferential configuration of arguments; namely, the deep structure of reasoning underlying the connection between a standpoint and the argument(s) in its support (Rigotti and Greco Morasso, 2009). The general principle underlying the reconstruction of the inferential configuration of argumentation is that of finding those implicit premises that are necessary for the argumentation to be valid.

In the AMT, two fundamental components should be distinguished when bringing to light the inferential relation binding the premises to the conclusion of an argumentation. First, an argument envisages a topical component, which focuses on the inferential connection activated by the argument, corresponding to the abstract reasoning that justifies the passage from the premises (arguments) to the conclusion (standpoint). The inferential connection underlying the argument is named with the traditional term maxim. Maxims are inferential connections generated by a certain semantic ontological domain named locus. Second, an endoxical component, which consists of the implicit or explicit material premises shared by the discussants that, combined with the topical component, ground the standpoint. These premises include endoxa, i.e., general principles, values, and assumptions that typically belong to the specific context, and data, basically coinciding with punctual information and facts regarding the specific situation at hand and usually representing the part of the argument that is made explicit in the text (Rigotti and Greco Morasso, 2011). Despite its particular concern for the inferential aspects of argumentation, the AMT accounts not only for the logical aspects of the argumentative exchange, but also for its embeddedness in the parties' relationship, and thus proves to be particularly suited for the argumentative analysis of public discourses.

In the present paper, we refer to the AMT to reconstruct the inferential structure of some arguments proposed by the two above-mentioned discourses, i.e., the type of reasoning underneath the arguments. In this sense, the model is assumed to be a guiding framework for the analysis, since it provides the criteria for the investigation of argumentative positions and for the identification of different components of each discourse. It is used to highlight points of contention and dialogue, as well as the explicit and implicit arguments advanced by the involved sustainers of the two narratives. The application of this analytic method in the study of public discourses, such as the role of inclusive education, is assumed to reinforce the possibilities of understanding how people discursively position themselves as involved partners in the management of the selected issue, namely, inclusive education.

\section{RESULTS}

According to the AMT, the following analytical components must be identified as: the maxim on which the argumentation is based and the relative locus at work; the endoxon, i.e., 
the premises shared by the discussants, and the datum, i.e., the punctual information and facts regarding the specific situation at hand (usually representing the part of the argument that is made explicit in the text) to which the argument is linked. The results of the AMT's reconstruction will be represented through graphical tools adopted to show the above-mentioned components.

Generally speaking, the different arguments used by the parties can be viewed in terms of a constellation of features (Goodwin, 2006), including various interactional structures connected to aims, perceptions, directives, accounts, etc. In the present paper, we will limit our conceptual analysis of two narratives to some elements that are essential for the aim of the study, although we are aware that this is a partial choice. Accordingly, the locus at work for the maxims will not appear in our schemes and only the arguments sustaining the main ideal view of each narrative and the presumed positions associated with the selected arguments will be presented.

In the next sections, we propose two examples of AMT based on selected arguments for each discourse.

\section{Reconstructing the Inferential Structure of the First Discourse Argument}

The first discourse ("inclusion for some") proposes as a standpoint that students with special needs require specialized educational settings. The argument advanced to sustain this position is that specialized settings are accommodating to the student's capacities and needs.

Figure 1 shows the representation of such an argument based on the AMT. On the right hand of the diagram, the inferential principle, i.e., the maxim, on which the argumentation is based is specified as: "to provide a beneficial property to the student, it is required to adopt a system that guarantees this beneficial property." The AMT representation allows consideration of the contextual premises that are implicitly or explicitly used in argumentation. This may be found on the left hand of the diagram, where a second line of reasoning is developed that supports the former one. This is why the first conclusion on the left becomes the minor premise on the right. In this way, the crossing of contextual and formal premises that is characteristic of argumentation is accounted for in the AMT. The endoxon refers in this case to common knowledge about the main idea of the accommodation principles: "To accommodate to the student's capacities is a beneficial property." The datum ("Specialized settings are accommodating to student capacities") combined with the endoxon produces the conclusion that "Specialized settings have beneficial properties."

In the first discourse, if the accommodation is considered beneficial for a student with special needs, and if specialized settings are recognized as environments that can guarantee a process of accommodation, then it is valuable to require that students with special needs should be placed in specialized settings.

\section{Reconstructing the Inferential Structure of the Second Discourse Argument}

The second discourse ("inclusion for all") proposes as a standpoint that all students require regular educational settings. The argument advanced to sustain this position should be summarized as follows: Regular settings offer equal opportunities to all students. Figure 2 shows the representation of such an argument based on the AMT.

On the right hand of the diagram, the inferential principle, i.e., the maxim, on which the argumentation is based is specified as: "if the offer of equal opportunities is an important educational goal, and there is a way to guarantee such a goal, then this way should be adopted." Concerning the contextual premises that are implicitly or explicitly used in argumentation, the endoxon refers to common knowledge about the main idea of the educational goals: "Education should offer equal opportunities to all students." The datum ("Regular settings offer equal opportunities to all students") combined with the endoxon produces the conclusion that "All students require regular educational settings."

The discourse indicates that if offering equal opportunities to all students (by exposing them to similar conditions) are considered an important educational goal, and if regular settings are recognized as environments that can guarantee to offer equal opportunities, then it is valuable to require that all students be placed in regular settings.

\section{Implicit in Two Discourses}

The models referring to the two discourses about inclusive education are showing in both cases reasonable arguments advanced to sustain the positions and the perspectives they intend to promote. For each discourse, accountable elements are proposed to show the pertinence of the approach and to sustain the idea of education that is considered as adequate for society.

The two discourses position the children as the main key-players in the educational endeavor: In fact, inclusive education should sustain the requirement for appropriate settings (special and regular) that are able to allow students (with and without special needs) to develop their capacities and to become members of the society. In this sense, the two discourses share a similar preoccupation and aim to play in the service of children's development. However, it is also true that both discourses promote reasons that seem to position the children within different frames, for example, in terms of temporality. In fact, the first discourse ("inclusion for some") focuses on the need to guarantee a process of accommodation to the children's needs in order to guarantee a system that allows students to develop their capacities. In this sense, a short-term perspective is promoted, because the goal behind the sustained discourse is to be able to act adequately in the "here and now" of the contingent situations. By contrast, the second discourse ("inclusion for all") advances the idea that offering equal opportunities to all students constitute the main goal of education. In this sense, a long-term perspective is promoted in terms of capacity to ensure the conditions that will guarantee 


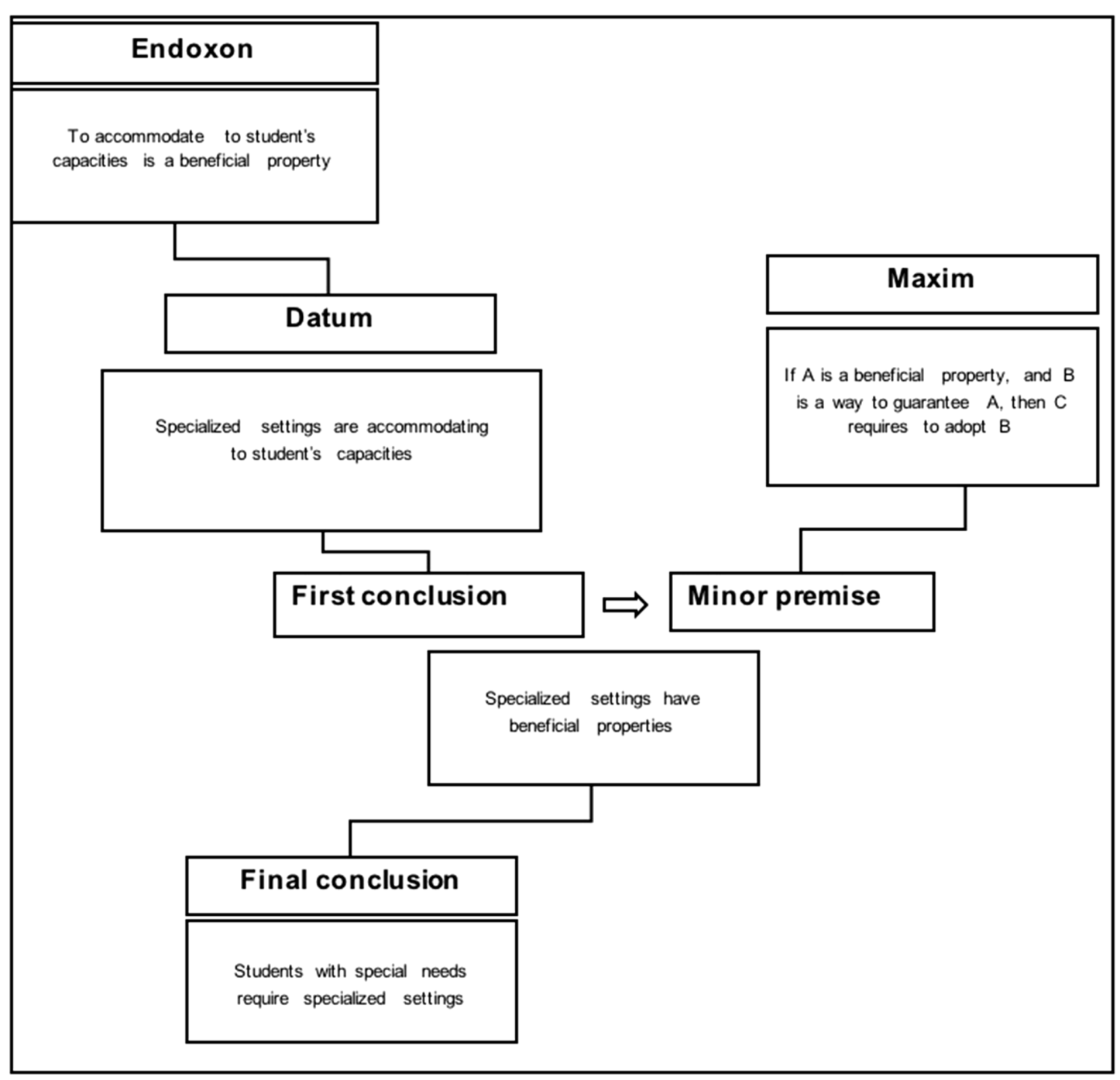

FIGURE 1 | AMT-based reconstruction of the first discourse argument.

the future realization of students as full members of the society. These elements, connecting the two discourses along a temporal dimension, will be discussed in the next section of the paper, as well as the implications in terms of positions that children and teachers should take according to their voice, the power relations that are connected to this, and the values that are enacted or prevented.

\section{DISCUSSION}

Although we have identified these two discourses in the current ongoing debate on inclusive education in Estonia, they encapsulated long lasting conflicting positions that can be recognized in many countries and communities. Moreover, these discourses also reflect political, policy, cultural, and identity "wars" that are present in many countries since the Salamanca Statement (UNESCO, 1994), calling societies to put forward the inclusive education on their education policy agenda. The "war" on inclusive education is related to the fact that educational policies are inherently political, since they always involve values, interests, power games, choices, prioritization, and allocation of resources (Barton, 1997). Moreover, different sides propose different values and ideals; that is, they postulate different desired outcomes and different visions of the future citizen (Magnússon, 2019). However, it is worth noting that current conflicting debates are just another step in an historical process of a struggle of regular schools between exclusion and inclusion of children and youth perceived and treated as different from dominant groups, in relation to various characteristics, such as socioeconomic status, gender, and race (Boroson, 2017). In fact, the meaningful inclusion of individuals who are different from the majority has been fraught in many ways. The evolution of educational systems with respect to the inclusion of students who are different in terms of race, gender, or ability was considered to be of questionable worth, an obstacle on teachers' 


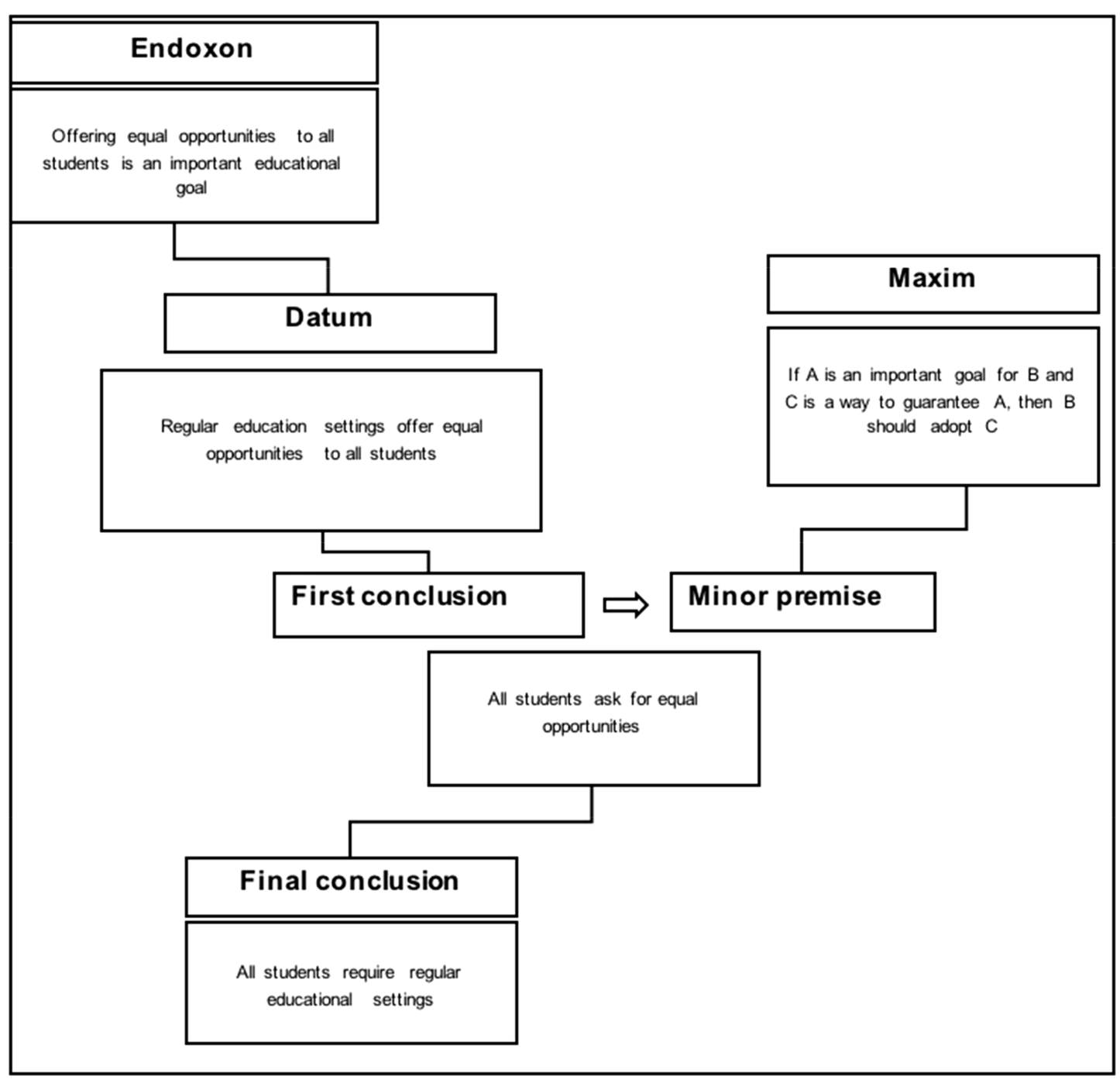

FIGURE 2 | AMT-based reconstruction of the second argument.

time and a threat to the status quo (West, 2000). Although today education in mainstream schools is guaranteed (Snyder et al., 2016), many educators and families still have a concern or even fear the "intrusion" into general education classrooms of students who are different than majority in terms of personal characteristics (physical, socioemotional, or cognitive) or ethnic, cultural, and socioeconomic background (Boroson, 2017). By considering the two discourses highlighted in this paper, we can consider that, on the one hand, those who are pro-specialist settings would argue that segregation works in favor of child with special education needs; on the other hand, those who view inclusion as a social justice issue might consider specialist settings as segregating like other forms of educational and historical segregation (e.g., gender and faith).

Two conclusions could be made from this. First, as with previous inclusion "wars," the current one will be resolved when conflicting sides will manage to dialogize their conflicting positions. The second conclusion is that the current "war" is just an episode in a continuous historical story on social inclusion, so after that one, there will be some new inclusion "war" that might not be imaginable from the perspective of our current experience and knowledge. Having said that, our main objective is to identify their frameworks in terms of assumptions, power relationships, voices, rights, and values, as well as priorities and practices in order to propose a bridging between them and to dialogize current relation that is dominantly conflicting.

As it is already said, both discourses put a stress on children's needs and recognize the duty of the education system to provide adequate conditions for their education. However, there is also an important difference in relation to the position and rights of children with special needs. The "inclusion for some" discourse recognizes the rights of children with special needs, but at the same time, it advocates that their rights need to be limited by practical constraints related to the implementation of the full inclusion in the regular school. In this way, this discourse 
gives a voice to children with special needs, but also to educational practitioners who are in many occasions not competent enough nor have adequate conditions and resources to ensure quality education to children with special needs in regular schools. Although both voices are represented in the discourse "inclusion for some," it prioritizes somewhat the voice of educational practitioners. On the other hand, the "inclusion for all" discourse privileges the voice of children with special needs and their rights that need to be served by the society in the same way as the rights of all other citizens. It also recognizes practical and policy constraints at the level of the education system, schools, and practitioners, but it does not position their voices and concerns at the same level as the rights of children with special needs. Thus, it advocates that schools and practitioners ought to be equipped by adequate policies, training, and resources to be capable of serving the rights of children with special needs for the quality education in inclusive conditions.

Difference in prioritization of voices is related to the difference in basic values and distribution of power. The discourse "inclusion for some" suggests that the current potential of the education system, schools, and teachers should be put at the first place and that rights of children with special needs should be realized progressively following the improvement of the potential of the education system to ensure high quality inclusive education. In this way, it gives more power to the majority, to the education system, and practitioners since it calls that rights on quality education need to be aligned with the potential of the education system to serve this right. However, in this way, it also creates an opportunity for using current lack of capacities in regular schools for ensuring inclusive education as a reason for postponing the realization of rights of children with special needs. If for some reason there is no political will or if the majority of educational practitioners is not willing to transform their beliefs, competences, and practices, then it might effectively maintain current conditions for some time (potentially endlessly). On the other hand, the "inclusion for all" discourse privileges the right of children with special needs over current conditions and lack of capacities and resources advocating that the latter needs to be transformed as quickly as possible. Consequently, it places higher power to the children with special needs and their fundamental rights than to eventual practical and political constraints of various kinds. Nevertheless, it might be related to some unintended negative consequence in the implementation of inclusive education. Forcing a full implementation of inclusive education when regular schools and practitioners are not prepared adequately might result in various negative consequences. These consequences might be counterproductive in terms of defending rights of children with special needs and effectively postpone the implementation of inclusive education. Therefore, in spite of differences in terms of basic values and power relations putting forward in two discourses, it is possible to identify a common interest. It is related to the successful implementation of inclusive education and the minimization of risk both for children with special needs and for education practitioners and schools including children without special needs.
Concerning the implementation of inclusive education, there are two opposite perspectives creating a major conflict between the two discourses. Being grounded on previous founding ideas, the "inclusion for some" advocates for some form of special education provision mostly in separate and specialized schools, while the "inclusion for all" discourse stands up for desegregation and full inclusion of children with special needs in regular schools. According to UNESCO (2020), the implications in developing forms of education that are effective for all children are related to three levels: educational (to develop ways of teaching that respond to individual differences and that therefore benefit all children), social (to change attitudes to difference by educating all children within a non-discriminatory society), and economic (it is likely to be less costly to establish and maintain schools).

These positions reflect their difference in terms of future priorities (Ydo, 2020). The "inclusion for some" discourse is focused to optimize provision of education as an ultimate goal. Hence, it prefers providing education in a specialized environment since it enables full accommodation to specific educational needs of children attending special schools. In this way, children with special needs might have best opportunities to learn in their way and to achieve education goals. On the other hand, the discourse "inclusion for all" calls for a more comprehensive ultimate goal. These goals ought to be to empower and enable children with special needs to become active citizens who will participate fully and equally in the society and to pursue their own life projects. Projecting this ultimate goal for education of children with special needs, the discourse "inclusion for all" pursues a full inclusion in regular schools since education in segregated institutions prevents children with special needs from becoming full members of the society. This difference in terms of the ultimate goal of education of children with special needs might seem as unresolvable. It also can make sense why the "war" between the two discourses and the communities organized upon them is very frequently concentrated on the special school issue. However, in our view, this opposition might be bridged by relating the two discourses to different time perspectives (as it has been already mentioned earlier). The common ground might be that all children with special needs are fully included in regular schools in order to enable and empower them to become active and equal future citizens, but to keep special schools and special education teachers as additional resources where different students from regular schools can get different forms of supplementary support according to their needs occasionally or in a longer period. This approach would require establishing a good and productive professional collaboration between regular and special schools as resource centers, as well as between teachers from regular schools and special education teachers. Based on a good professional collaboration and complementary professional competences of all teachers (including special education teachers), children with special needs would get an additional support during classes in regular schools or when it is needed in a special school (for example, when the child needs a specialized treatment or to get additional training for using some assistive technology). It is true that this arrangement could be challenged 
by some practical issues and would require a modification of regular institutional organization and practices. However, it would improve opportunities for children with special needs to become competent future citizens, and for the education system and the society to become inclusive.

Furthermore, additional common ground might be related to the pace of the long-term implementation of inclusive education. The discourse "inclusion for all" provides a strong argument why inclusive education is the principal way to empower and enable children with special needs to grow up with a feeling that they are equal members of the society and with a dignity to take part fully in the life of the community so to pursue their life projects and contribute to the society. However, the discourse "inclusion for some" pinpoints in a good way that journey toward the ultimate goal cannot be straightforward nor quick because it is related to the transformative potential of the society and the education system imposing important constraints. Although these constraints are malleable and temporary, they need to be addressed in any implementation plan for inclusive education. Therefore, we assume that the two discourses can be bridged in the sense that one of them crystalizes and advocates what ought to be long-term goals for the implementation of inclusive education, while the second one articulates practical constraints and barriers that need to be overcome in order to make inclusive education real.

\section{CONCLUSION}

In this paper, we utilized the AMT for analyzing two somewhat opposing discourses regarding inclusive education, namely, the "inclusion for some" and "inclusion for all." We reconstructed the inferential configurations of the arguments of each narrative, identified how the two definitions contribute to position children with and without special needs and their teachers. The results showed several similarities and differences between the discourses. We also identified some possibilities to bridge the two narratives;

\section{REFERENCES}

Ainscow, M., and César, M. (2006). Inclusive education ten years after Salamanca: setting the agenda. Eur. J. Psychol. Educ. 21, 231-238. doi: 10.1007/BF03173412

Ainscow, M., and Miles, S. (2008). Making education for all inclusive: where next? Prospects 38, 15-34. doi: 10.1007/s11125-008-9055-0

Arcidiacono, F., and Baucal, A. (2020). Towards teacher professionalization for inclusive education: reflections from the perspective of the socio-cultural approach. Eesti Haridusteaduste Ajakiri. Estonian J. Edu. 8, 26-47. doi: 10.12697/eha.2020.8.1.02b

Barton, L. (1997). Inclusive education: romantic, subversive or realistic? Int. J. Incl. Educ. 1, 231-242. doi: 10.1080/1360311970010301

Basic Schools and Upper Secondary Schools Act. (2010). Riigi Teataja I, 2010,41,240. Available at: http://riigiteataja.ee/en/eli/ee/530102013042/consolide (Accessed January 03, 2021).

Basic Schools and Upper Secondary Schools Act. (2019). Riigi Teataja I, 2019,120. Available at: http://riigiteataja.ee/en/eli/ee/Riigikogu/act/503062019007/consolide (Accessed January 03, 2021).

Boroson, B. (2017). Inclusive education: lessons from history. Educ. Leadersh. $74,18-23$. most importantly, by relating to different time perspectives, these two discourses stress: "Inclusion for some," which tends to focus on the present situation and attending to the particularities of the child, is valuable for realizing the longterm and sometimes idealistic goals of "inclusion for all" and vice versa, "inclusion for all," which stresses participation and learning with peers, is beneficial for realizing the goals of "inclusion for some" - to maximize each child's potential in real life - since regular schools resemble society more closely than segregated schools. Productive professional collaboration between different parties is required to realize both visions of inclusive education. We also suggest further investigations to deepen this research line in the future, through face-to-face interviews with politicians, school managers, teachers, and parents who could better delineate the different positions according to their role and involvement with children with special needs.

\section{AUTHOR CONTRIBUTIONS}

All the authors originated the paper's ideas and designed the conceptual analysis. ÄL was engaged in presenting the context of the study, as well as interpreting and discussing the findings and writing the conclusion. FA focused on the method, analysis, and interpretation of the narratives. $A B$ focused mainly on interpreting and discussing the findings. All authors approved the final version of the manuscript to be published.

\section{FUNDING}

This research was conducted with financial support by the European Regional Development Fund under Grant Enhancement of Research and Development Capacity of Teacher Education Competence Centre Pedagogicum [grant no. NSVHI19074], as well as the contribution of the Research Department, University of Teacher Education BEJUNE (Switzerland).

Breeman, L. D., Wubbels, T., van Lier, P. A. C., Verhulst, F. C., van der Ende, J., Maras, A., et al. (2015). Teacher characteristics, social classroom relationships, and children's social, emotional, and behavioral classroom adjustment in special education. J. Sch. Psychol. 53, 87-103. doi: 10.1016/j.jsp.2014.11.005

Carrington, S. (1999). Inclusion needs a different school culture. Int. J. Incl. Educ. 3, 257-268. doi: 10.1080/136031199285039

Cigman, R. (2007). A question of universality: inclusive education and the principle of respect. J. Philos. Educ. 41, 775-793. doi: 10.1111/j.1467-9752.2007.00577.x

Croll, P., and Moses, D. (2000). Ideologies and utopias: education professionals views of inclusion. Eur. J. Spec. Needs Educ. 15, 1-12. doi: $10.1080 / 088562500361664$

Dyson, A., Howes, A., and Roberts, B. (2002). A Systematic Review of the Effectiveness of School-Level Actions for Promoting Participation by all Students. Inclusive Education Review Group, EPPI-Centre.

Ehala, M. (2020). Tulevik on erikoolide päralt. [Future is for special schools]. Postimees. Available at: https://leht.postimees.ee/6901395/tulevik-on-erikoolide-paralt (Accessed January 03, 2021).

Farrell, M. (2010). Debating Special Education. London: Routledge. 
Felder, F. (2019). Inclusive education, the dilemma of identity and the common good. Theory Res. Educ. 17, 213-228. doi: 10.1177/1477878519871429

Florian, L., and Becirevic, M. (2011). Challenges for teachers' professional learning for inclusive education in central and Eastern Europe and the commonwealth of independent states. Prospects 41, 371-384. doi: 10.1007/ s11125-011-9208-4

Freeman, S. F. N., and Alkin, M. C. (2000). Academic and social attainments of children with mental retardation in general education and special education settings. Remedial Spec. Educ. 21, 3-26. doi: 10.1177/074193250002100102

Goodwin, M. H. (2006). Participation, affect, and trajectory in family directive/ response sequences. Text Talk 26, 515-543. doi: 10.1515/TEXT.2006.021

Haug, P. (2017). Understanding inclusive education: ideals and reality. Scand. J. Disabil. Res. 19, 206-217. doi: 10.1080/15017419.2016.1224778

Hornby, G. (2015). Inclusive special education: evidence-based practices for children with special needs and disabilities. British J. Special Educ. 42, 232-252. doi: 10.1111/1467-8578.12101

Kauffman, J. M., and Hornby, G. (2020). Inclusive vision versus special education reality. Educ. Sci. 10:258. doi: 10.3390/educsci10090258

Kivirand, T., Leijen, Ä., Lepp, L., and Malva, L. (2020). Kaasava hariduse tähendus ja tõhusa rakendamise tegurid Eesti kontekstis: õpetajaid koolitavate või nõustavate spetsialistide vaade [The meaning of inclusive education and factors for effective implementation in the Estonian context: A view of specialists who train or advise teachers]. Eesti Haridusteaduste Ajakiri. Estonian J. Educ. 8, 48-71. doi: 10.12697/eha.2020.8.1.03

Kivirand, T., Leijen, Ä., Lepp, L., and Tammemäe, T. (2021). Designing and implementing an in-service training course for school teams on inclusive education: reflections from participants. Educ. Sci. 11:166. doi: 10.3390/ educsci11040166

Koller, D., Le Pouesard, M., and Rummens, J. A. (2018). Defining social inclusion for children with disabilities: A critical literature review. Child. Soc. 32, 1-13. doi: $10.1111 /$ chso.12223

Konetski-Ramul, K. (2021). Kaasav haridus ehk Elevant keset tuba. [Inclusive education as an Elephant in the room.] Oppetajate Leht. https://opleht. ee/2021/02/kaasav-haridus-ehk-elevant-keset-tuba/ (Accessed January 03, 2021).

Koutsouris, G., Anglin-Jaffe, H., and Stentiford, L. (2020). How well do we understand social inclusion in education? Br. J. Educ. Stud. 68, 179-196. doi: $10.1080 / 00071005.2019 .1658861$

Kupper, B., Õun, M., Tatomir, T., and Simso, T. (2020). Haridusinnovatsioon: kaasav haridus - kas oleme ikka kõigele mõelnud? [Educational innovation: Have we really thought abouteverything?] Postimees. https://arvamus.postimees. ee/6999120/haridusinnovatsioon-kaasav-haridus-kas-oleme-ikka-koigele-moelnud (Accessed January 03, 2021).

Labi, N. (2019). Kaasavast haridusest on kasu kõigile [Inclusive education is beneficial for everyone.] Õpetajate Leht. https://opleht.ee/2019/05/kaasavastharidusest-on-kasu-koigile/ (Accessed January 03, 2021).

Leijen, Ä., and Pedaste, M. (2018). "Pedagogical beliefs, instructional practices, and opportunities for professional development of teachers in Estonia" in The Teacher's Role in the Changing Globalizing World. eds. H. Niemi, A. Toom, A. Kallioniemi and J. Lavonen (Leiden, Netherlands: BRILL), 33-46.

Magnússon, G. (2019). An amalgam of ideals - images of inclusion in the Salamanca statement. Int. J. Incl. Educ. 23, 677-690. doi: 10.1080/13603116.2019.1622805

Nelis, P., and Pedaste, M. (2020). Kaasava hariduse mudel alushariduse kontekstis: süstemaatiline kirjandusülevaade [A model of inclusive education in the context of Estonian preschool education: A systematic literature review]. Eesti Haridusteaduste Ajakiri. Estonian J. Educ. 8, 138-163. doi: 10.12697/eha.2020.8.2.06

OECD. (2007). Students with Disabilities, Learning Difficulties and Disadvantages Policies. Statistics and Indicators. Paris: OECD Publishing.

OECD. (2014). TALIS 2013 Results: An International Perspective on Teaching and Learning, TALIS. Paris: OECD Publishing.

OECD. (2020). TALIS 2018 Results: Teachers and School Leaders as Valued Professionals. Vol. 2 Paris: OECD Publishing.

Radó, P., Setenyi, J., Petrović, D., Stanković, D., and Markovits, G. (2016). Formative Evaluation of Implementation of Inclusive Practices in the Education
System in Serbia (2009-2014). UNICEF. https://evaluationreports.unicef.org/ GetDocument?fileID=8922 (Accessed January 03, 2021).

Räis, M.-L., Kallaste, M., and Sandre, S.-L. (2016). Haridusliku erivajadusega õpilaste kaasava hariduskorralduse ja sellega seotud meetmete tõhusus. Lõppraport [Effectiveness of inclusive education arrangements and related measures for students with special educational needs. Final report]. Eesti Rakendusuuringute Keskus CENTAR [Estonian Centre for Applied Research CENTAR].

Räis, M.-L., and Sõmer, M. (2016). Haridusliku erivajadusega õpilaste kaasava hariduskorralduse ja sellega seotud meetmete tõhusus. Temaatiline raport kaasamise tähenduslikkus [Effectiveness of inclusive education organization and support for students with special educational needs. Thematic report: The meaning of inclusion]. Eesti Rakendusuuringute Keskus CENTAR [Estonian Centre for Applied Research CENTAR].

Rigotti, E., and Greco Morasso, S. (2009). "Argumentation as an object of interest and as a social and cultural resource," in Argumentation and Education. Theoretical Foundations and Practices. eds. N. Muller Mirza and A.-N. PerretClermont (New York: Springer), 9-66.

Rigotti, E., and Greco Morasso, S. (2011). Comparing the argumentum model of topics to other contemporary approaches to argument schemes: the procedural and material components. Argumentation 24, 489-512. doi: 10.1007/ s10503-010-9190-7

Snyder, T. D., de Brey, C., and Dillow, S. A. (2016). Digest of Education Statistics 2014 (NCES 2016-006). Washington: National Center for Education Statistics, Institute of Education Sciences, U.S. Department of Education.

Stepaniuk, I. (2019). Inclusive education in eastern European countries: a current state and future directions. Int. J. Incl. Educ. 23, 328-352. doi: $10.1080 / 13603116.2018 .1430180$

UN. (1989). Convention on the Rights of the Child. https://treaties.un.org/doc/ Treaties/1990/09/19900902\%2003-14\%20AM/Ch_IV_11p.pdf (Accessed January 03, 2021).

UNESCO. (1994). Salamanca Statement and Framework for Action on Special Needs Education. Available at: https://unesdoc.unesco.org/ark/48223/pf0000098427 (Accessed January 03, 2021).

UNESCO (2020). "Towards inclusion in education: status, trends and challenges," in The UNESCO Salamanca Statement 25 Years on (Paris: UNESCO).

Van Mieghem, A., Verschueren, K., Petry, K., and Struyf, E. (2018). An analysis of research on inclusive education: a systematic search and meta review. Int. J. Incl. Educ. 24, 675-689. doi: 10.1080/13603116.2018.1482012

West, J. (2000). Back to School on Civil Rights: Advancing the Federal Commitment to Leave no Child Behind. Washington: National Council on Disability.

Ydo, Y. (2020). Inclusive education: global priority, collective responsibility. Prospects 49, 97-101. doi: 10.1007/s11125-020-09520-y

Zgaga, P. (2019). "Inclusion in education: Between opportunities and limits. Introduction to the theme," in Inclusion in Education: Reconsidering Limits, Identifying Possibilities (Berlin: Peter Lang), 7-28.

Conflict of Interest: The authors declare that the research was conducted in the absence of any commercial or financial relationships that could be construed as a potential conflict of interest.

Publisher's Note: All claims expressed in this article are solely those of the authors and do not necessarily represent those of their affiliated organizations, or those of the publisher, the editors and the reviewers. Any product that may be evaluated in this article, or claim that may be made by its manufacturer, is not guaranteed or endorsed by the publisher.

Copyright (C) 2021 Leijen, Arcidiacono and Baucal. This is an open-access article distributed under the terms of the Creative Commons Attribution License (CC BY). The use, distribution or reproduction in other forums is permitted, provided the original author(s) and the copyright owner(s) are credited and that the original publication in this journal is cited, in accordance with accepted academic practice. No use, distribution or reproduction is permitted which does not comply with these terms. 


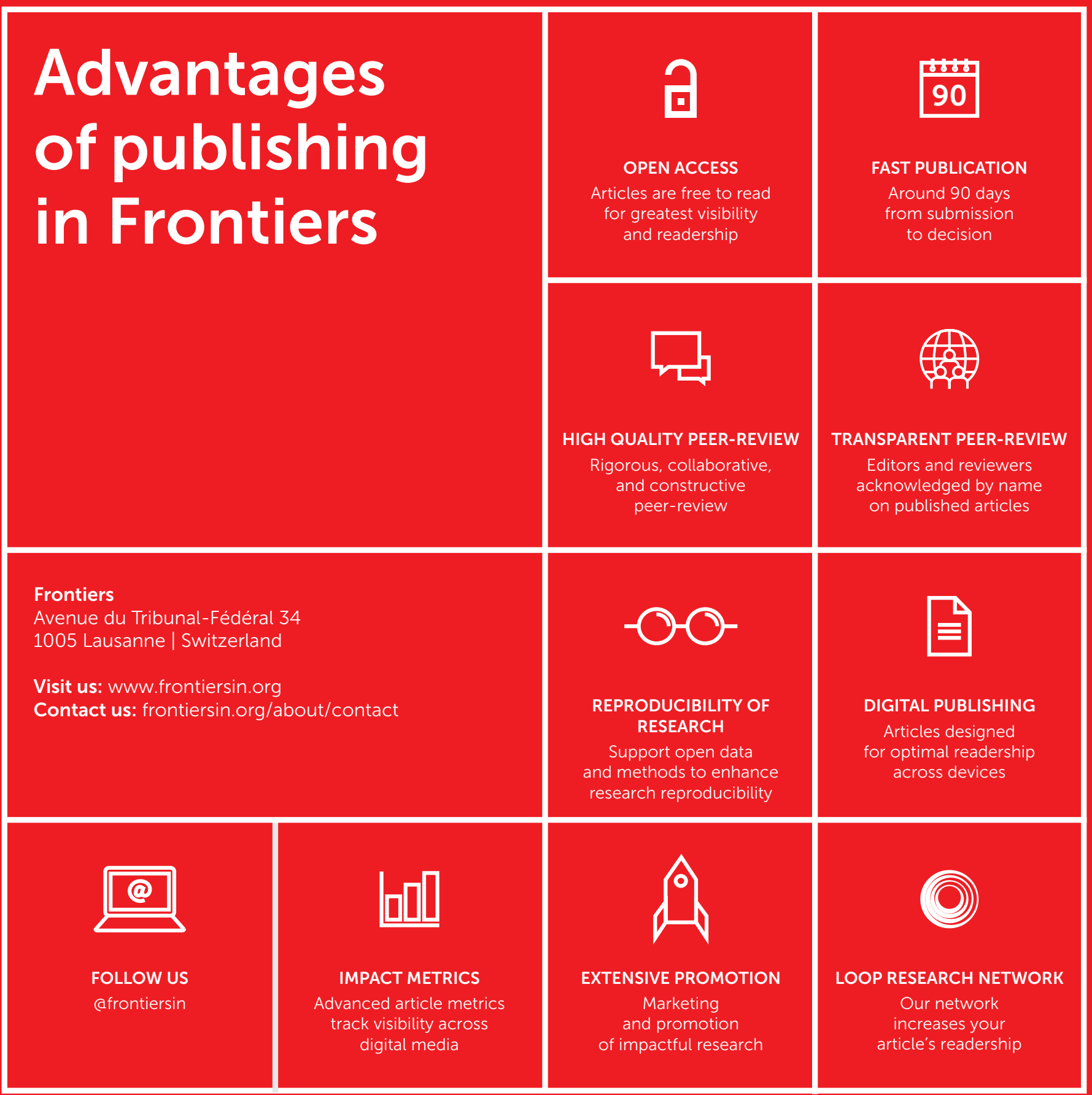

